FERMILAB-THESIS-2007-103

\title{
The Search For New Physics in Di-Electron Events at $\mathrm{CDF}$
}

\author{
Sam Harper \\ New College, Oxford
}

Thesis submitted in fulfilment of the requirements for the degree of Doctor of Philosophy at the University of Oxford

Michaelmas Term, 2006 


\title{
The Search For New Physics in Di-Electron Events at $\mathrm{CDF}$
}

\author{
Sam Harper \\ New College, Oxford \\ Thesis submitted in fulfilment of the requirements for the degree of Doctor of \\ Philosophy at the University of Oxford \\ Michaelmas Term, 2006
}

\begin{abstract}
A narrow resonance resulting from a new physics process is searched in the dielectron channel using approximately $819 \mathrm{pb}^{-1}$ of Tevatron proton-antiproton collision data at $\sqrt{s}=1.96 \mathrm{TeV}$. No significant evidence for such a narrow resonance is found in the search range of $150-950 \mathrm{GeV} / c^{2}$. Limits are set on the $\sigma \cdot B r\left(X \rightarrow e^{+} e^{-}\right)$at the $95 \%$ confidence level for neutral spin-1 and spin-2 particles in the mass range from $200-950 \mathrm{GeV} / c^{2}$. These limits are used to exclude the $E_{6} Z^{\prime}$ bosons; $Z_{I}^{\prime}, Z_{\psi}^{\prime}, Z_{\chi}^{\prime}$ and $Z_{\eta}^{\prime}$ with masses below 694, 779, 782 and $847 \mathrm{GeV} / c^{2}$ respectively. The Randall-Sundrum graviton is also excluded with a mass below $764(230) \mathrm{GeV} / c^{2}$ for $k / \bar{M}_{p l}=0.1(0.01)$. The di-photon channel is then combined with the di-electron channel to increase the Randall-Sundrum graviton exclusion limits to 874 (244) $\mathrm{GeV} / c^{2}$ for $k / \bar{M}_{p l}=0.1(0.01)$.
\end{abstract}


To my parents who have always been there for me, have given me every opportunity in life and have always been completely supportive of my choices. 


\section{Acknowledgements}

The road to this point has long been indeed. It seems an eternity since I first stepped on the path to career in high energy physics. Along this road many have guided me and many have made the path a little easier to tread and for this I am thankful to them. I have far too little time to thank them all properly but I will try. First, my thanks to Costas Foudas, who first started me on this road with a series of undergraduate projects and was always an enthusiastic supervisor. On my acknowledgements in my MSci project he wrote something along the lines of, "will you still be thanking me or cursing me in 3 years time". Hmm, well, the jury was out for a little while but on the whole, yes.

My thanks to my supervisors, Peter Renton and Joel Goldstein for always being available to help. A fast finish is always indicative of the level of support a student receives and for this I thank you. I really appreciate all the time you spent helping me with this and for often traveling to Fermilab to help out (and also for suffering my atrocious spelling when checking the drafts of this thesis). My thanks for also helping me weather the unpleasant political realities of HEP. Next I must absolutely thank Beate Heinemann. If it wasn't for her tireless help, this thesis would be a pale shadow of its current form. Always available no matter how busy she was, and always trying to help sort out a situation, Beate is truly an amazing person and one who many graduate students at CDF, myself especially, owe a lot to. I think its safe to say I might already have left the field if it wasn't for her support. I am also extremely thankful to Rob Roser for all the support and help he has given to me and also my thanks goes to Jacobo Konigsberg for his support as well. My thanks also goes out to Greg Veramendi and Catalin Ciobanu for being such excellent people to work with and for teaching me much about how to do a HEP analysis. Larry Nodulman and Kaori Maeshima were also inspirational sources of help and their experience was a fantastic asset to be able to call upon. To the conveners whose insightful comments and sharp eyes made this analysis much better than it would have been; Ben Brau, Amit Lath, Alexei Safonov, Jane Nachtman, Chris Hays and Beate (again). To Ray Culbertson for all the help with the Stntuples. To Müge for being really helpful when writing up and for always being full of energy, which seems to have a contagious effect. And to the rest of the Oxford CDF group; Aidan, Chris, Farrukh, Jacob, Jonas, Jorgen, Lucio, Louis, Louise, Matt, Nicola, Oliver, Sneha and Todd for such an enjoyable working environment. Also I must thank Beverly, Sue and Kim for all their behind the scenes work helping things run smoothly and shielding all us DPhil students from all the bureaucratic red-tape, 
letting us get on with actually doing the thesis instead of filling in forms all the time.

Other people have made my road just that much easier. My flatmates at Imperial; Kev, Dan, Katie, Steve, Jane, Hannah, Nikki and Keith who made the four years of undergraduate work leading up to this such fun. My fellow students at Oxford who made my time at Oxford so enjoyable: Tobi, Phil, Nick, Alys, Dave, Ryan, Justin and Laura. DC breaks just weren't quite the same in Chicago without you Laura. In Chicago, the UK Fermi Posse were a source of great fun and a much needed counter balance to work. You all really made my two years in Chicago such an amazing time and I thank you Martin G., Nicola, Smudge, Mark W., James W., Sneha, Troy, Sinead, Phil V., Emily, Matt T., Matt L. James M., Marilyn, Toby, Mark O., Gavin, Tamsin, Lydia, Amber, Ben, Marcus, Jacob, Stephen and Sarah. In particularly my thanks goes to my flatmates in the 'Bachelor's Pad', Mark W. and James W. for making the second year so much better than the first. To Nicola always cheering up the Oxford office and making it a much more pleasant place to work in (and also for nagging me to get this done). To Sneha for also making my office a much more pleasant place to work and for being so generous with her flat. To Smudge for always brightening up my day and for being such a good listener. To Phil V. for always being a source of procrastination on messenger. To Sinead for many enjoyable conversations. And to Troy for being such a free spirit and reminding us all there's more to life than physics.

And, shock horror, I also meet some people out in Chicago who weren't British particle physicists and they also made my time in Chicago all the richer; Kirby (even if you do support Duke and enjoy Robbie Fulks, my eye still twinges every year around Halloween), Kathy (so many good times at the Michigan house), Bo (its always good to meet somebody who does appreciate a good cup of tea), Nate (although images from that murder mystery party still haunt me to this day), Dean (although you made bad call swapping California weather for the Chicago winter) and Joey and Sarah (so many fun nights out in Chicago, with the possible exception of the Foot).

Also many thanks to Michelle, Chris and Robin at the Alliance Bakery for performing the vital job of keeping me adequately caffeinated and nourished during the majority of the writing period of this thesis and in whose bakery a significant portion of this thesis was written. And to the fine folks at Macs, the place of my late afternoon office although the quality of the sections written there after a few hours were unfortunately not as high as the others.

To PPARC for funding me to do this DPhil and for generously allowing me to spend 2 amazing years in Chicago. My thanks to my examiners Peter Watkins and Mandy Cooper-Sarker for both managing to wade through this thesis and for an enjoyable discussion of it.

And finally to my parents. Without such loving and supportive parents, I would never be where I am now. They have given me every opportunity in life and for this I am profoundly grateful. Thank you. 


\section{Contents}

$\begin{array}{lll}1 & \text { Introduction } & 1\end{array}$

1.1 The Standard Model . . . . . . . . . . . . . . . . . . . . . . 1

1.1.1 Particles of the Standard Model . . . . . . . . . . . . . . 2

1.1.2 Standard Model Interactions . . . . . . . . . . . . . . . . . 2

1.1.3 Di-Electron Production in the Standard Model . . . . . . . . . . 6

1.1.4 Problems with the Standard Model . . . . . . . . . . . 8

1.2 New Phvsics Bevond The Standard Model . . . . . . . . . . . . . . . 10

1.2.1 Additional $U(1)$ Gauge Svmmetries . . . . . . . . . . . . 11

1.2 .2 Technicolour. . . . . . . . . . . . . . . . . . . . . 11

1.2 .3 Warped Extra Dimensions . . . . . . . . . . . . . . . . . 13

1.3 Analvsis Strategy . . . . . . . . . . . . . . . . . . . . . . . . 14

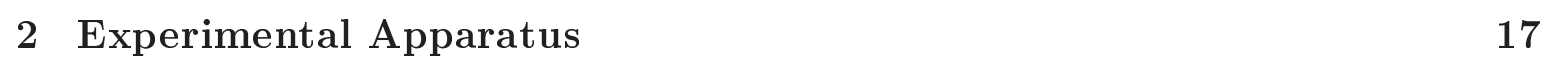

2.1 The Fermilab Accelerator Complex . . . . . . . . . . . . . . . . 17

2.2 The CDF II Detector . . . . . . . . . . . . . . . . . . . . . 21

2.2 .1 CDF Coordinate System . . . . . . . . . . . . . . 21

2.2 .2 Tracking Svstem . . . . . . . . . . . . . . . . 23

2.2 .3 Calorimeter Svstem . . . . . . . . . . . . . . . . . 25

2.2 .4 Muon Svstem . . . . . . . . . . . . . . . . . . . . . . 29

2.2 .5 Luminositv Monitoring . . . . . . . . . . . . . . . . 30

2.2 .6 Trigger Svstem . . . . . . . . . . . . . . . . . . 30

2.2 .7 Offline Processing and Software . . . . . . . . . . . . . 33

3 Triggers and Datasets 34

3.1 Data Sample. . . . . . . . . . . . . . . . . . . . . . . . . 34

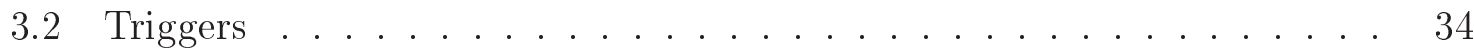

3.3 Monte Carlo Samples . . . . . . . . . . . . . . . . . . . . . . 35

$4 \quad$ Electron Identification and Event Selection 39

4.1 Central Electron Reconstruction and Identification . . . . . . . . . 40

4.1 .1 Central Electron Reconstruction . . . . . . . . . . . . . . . . . 40

4.1 .2 Central Electron Energy Scale . . . . . . . . . . . . . . 41

4.1 .3 Tower 9 Events . . . . . . . . . . . . . . . . . 43 
4.1 .4 Central Electron Identification . . . . . . . . . . . . . . . . 47

4.2 Plug Electron Reconstruction and Identification . . . . . . . . . . . . . 49

4.2.1 Plug Electron Reconstruction . . . . . . . . . . . . . . . . . . 49

4.2 .2 Plug Energv Scale . . . . . . . . . . . . . . . . . . 50

4.2 .3 Phoenix Tracking . . . . . . . . . . . . . . . . . 51

4.2 .4 Plug Electron Identifications Variables . . . . . . . . . . . . . . 54

5 Electron Identification Efficiency and Acceptance 57

5.1 Electron Identification Efficiency . . . . . . . . . . . . . . . 57

5.1 .1 Method used to Measure the Electron ID Efficiency . . . . . . . 58

5.1 .2 Background Estimation . . . . . . . . . . . . . 60

5.1 .3 Svstematic Uncertainties on the Identification Efficiency . . . 63

5.2 Kinematic and Geometrical Acceptance . . . . . . . . . . . . 63

5.3 Acceptance and Efficiency Estimate Cross-Check . . . . . . . . . 66

6 Standard Model Backgrounds and Cross-Checks $\quad 69$

$6.1 Z^{0} / \gamma^{*} \rightarrow e^{+} e^{-}$Background . . . . . . . . . . . . . 69

6.2 Jet Background . . . . . . . . . . . . . . . . . . . . 70

6.2 .1 Isolation vs Isolation Normalisation Method . . . . . . . . 70

6.2 .2 Jet Background Shape . . . . . . . . . . . . . . . . . . 77

6.3 Di-Photon Background . . . . . . . . . . . . . . . . . . . . . 79

6.4 Electroweak Processes Background . . . . . . . . . . . . . . . . 79

6.5 Non-Drell-Yan Background Cross-Check . . . . . . . . . . . . 79

\begin{tabular}{ll|l}
7 & Results & 86
\end{tabular}

7.1 New Phvsics Search . . . . . . . . . . . . . . . . . . . . . 88

7.2 Limits on New Phvsics . . . . . . . . . . . . . . . . . . . . 92

7.2.1 Procedure for Setting Limits on New Phvsics Processes . . . . . 95

7.2 .2 Procedure for Including Svstematic Uncertainties . . . . . . . . 96

7.2 .3 Expected and Observed Limits . . . . . . . . . . . . . 97

7.2 .4 Model Specific Limits . . . . . . . . . . . . . . . . . . . . . . 99

7.2 .5 Combination with the Di-Photon Channel . . . . . . . . . . . 107

8 Conclusions 113

$8.1 \quad$ Future Prospects . . . . . . . . . . . . . . . . . . . . . . . . . . 113

\begin{tabular}{|lr}
\hline A Electron Identification Cut Selection & 115
\end{tabular}

A.1 Central Electron Identification Cuts . . . . . . . . . . . . . . . . . 118

A.2 Plug Electron Identification Cuts . . . . . . . . . . . . . . 124

\begin{tabular}{ll}
\hline Bibliography & 130
\end{tabular} 


\section{List of Figures}

1.1 The Standard Model Drell-Yan process at LO in $p \bar{p}$ collisions . . . . . . 7

1.2 Next-to-leading order contributions to the SM Drell-Yan Process . . . . 7

1.3 Non Drell-Yan processes which can produce two final state leptons . . . 8

2.1 The Fermilab accelerator chain . . . . . . . . . . . . . . . . . . . 19

2.2 The delivered luminositv vs time . . . . . . . . . . . . . . . 20

2.3 A side-on schematic view of the CDF II detector . . . . . . . . . . . 21

2.4 The CDF coordinate svstem . . . . . . . . . . . . . . 22

2.5 An end-on view of the CDF silicon system . . . . . . . . . . . . . . . 24

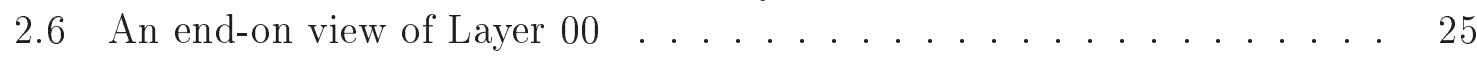

2.7 One eight of the east COT end-plate showing the superlaver lavout . $\quad 26$

2.8 The tower segmentation of the plug calorimeter . . . . . . . . . . . 29

2.9 A flow diagram of the CDF trigger system. . . . . . . . . . . . 32

3.1 The Z_NOTRACK trigger efficiency for central-plug events . . . . . . . . 36

4.1 The central-central mass spectrum at the $Z^{0}$ pole . . . . . . . . . . . 42

4.2 Comparision of data and MC mass scales in sub-regions of the CEM . . 44

4.3 Comparison of central-central data and MC mass scales vs energy . . . 45

4.4 The central-central mass spectrum for tower 9 events . . . . . . . . 46

4.5 The central-plug mass spectrum at the $Z^{0}$ pole . . . . . . . . . . . 52

4.6 Comparison of data and MC mass scales in sub-regions of the PEM . . 53

4.7 Comparison of central-plug data and MC mass scales vs energy . . . . 54

5.1 Estimated efficiencies at high mass . . . . . . . . . . . . . 61

5.2 Estimated acceptances for spin-1 and spin-2 particles . . . . . . . . . 66

5.3 Total estimated selection efficiencies for spin-1 and spin-2 particles . . . 67

6.1 The predicted Drell-Yan mass spectra for the CC and CP channels . . 71

6.2 Electron $E_{T}^{c o r r}$ vs electron $E_{T}^{c o r r} \ldots \ldots \ldots \ldots \ldots$. . . . . . . . . . 72

6.3 Plug electron isolation $E_{T}$ for data and MC simulated events . . . . . . 76

6.4 The ratio of electron/iet reconstructed $E_{T}$ for a jet . . . . . . . . . 80

6.5 The estimated shape of the jet background . . . . . . . . . . . 81

6.6 The estimated shape of the jet background and its uncertainty . . . . . 82

6.7 The anti-Phoenix selected central-plug mass spectrum . . . . . . . . . . 84 
6.8 The anti-conversion selected central-plug mass spectrum . . . . . . 85

7.1 The di-electron mass spectra for the $\mathrm{CC}$ and $\mathrm{CP}$ channels . . . . . . . . 90

7.2 The di-electron mass spectrum for the $\mathrm{CC}$ and $\mathrm{CP}$ channels combined . 91

7.3 The p-value spectrum for the $\mathrm{CC}$ and $\mathrm{CP}$ channels combined . . . . . . 93

7.4 The p-value spectra for the CC and CP channels separately . . . . . 94

7.5 The pull of the cross-section fit for $500 \mathrm{GeV} / c^{2} Z^{\prime} \ldots \ldots 6$

7.6 Likelihood distributions for spin-1 particles . . . . . . . . . . . . . 98

7.7 Expected limits for a spin-1 particle . . . . . . . . . . . . . . . . 100

7.8 Expected limits for a spin-2 particle . . . . . . . . . . . . . . . . . 101

$7.995 \%$ CL limits on $\sigma \cdot \operatorname{Br}\left(Z^{\prime} \rightarrow e^{+} e^{-}\right) \ldots \ldots \ldots \ldots$

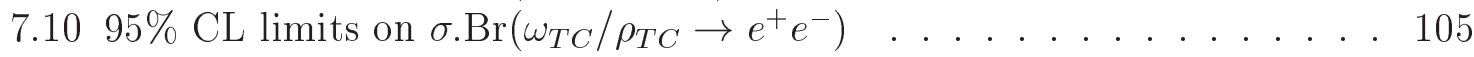

$7.1195 \%$ CL limits on $\sigma \cdot \operatorname{Br}\left(G_{R S} \rightarrow e^{+} e^{-}\right) \ldots \ldots \ldots \ldots$. . . . . . . 106

7.12 Comparison of limits using different treatments of PDF uncertainties . 111

$7.1395 \%$ CL limits on $\sigma \cdot \operatorname{Br}\left(G_{B S} \rightarrow l^{+} l^{-}\right)$for the $e^{+} e^{-}+\gamma \gamma$ channels . . . . 112

A.1 Electron isolation $E_{T}$ vs $E_{T} \ldots \ldots \ldots \ldots \ldots \ldots \ldots$

A.2 The N-1 efficiency and bkg. rejection of the CEM Isol. $E_{T}$ cut . . . . . 119

A.3 The N-1 efficiency and bkg. rejection of the CEM E/p cut . . . . . . . 120

A.4 The N-1 efficiency and bkg. rejection of the conversion cut . . . . . . . 121

A.5 The N-1 efficiency and bkg. rejection of the $\chi_{\text {and }}^{2}$ cut . . . . . . . . 122

A.6 The N-1 efficiency and bkg. rejection of the $L_{S h r}$ cut . . . . . . . . . . 122

A.7 The N-1 efficiency and bkg. rejection of the CES $\triangle X$ cut . . . . . . . . 123

A.8 The N-1 efficiency and bkg. rejection of the CES $\Delta Z$ cut . . . . . . . . 123

A.9 The N-1 efficiency and bkg. rejection of the PEM Isol. $E_{T}$ cut . . . . 125

A.10 The N-1 efficiency and bkg. rejection of the PEM $\chi_{2 \times 2}^{2}$ cut . . . . . 125

A.11 The N-1 efficiency and bkg. rejection of the PES $5 \times 9 \mathrm{U} / \mathrm{V}$ cut . . . 126

A.12 The N-1 efficiency and bkg. rejection of the PEM $\Delta R$ cut . . . . . . 127

A.13 The fraction of jet and di-photon events to the total bkg and signal . 129 


\section{List of Tables}

1.1 Lepton charges, spins and masses . . . . . . . . . . . . . . . . . . . . . . . . . . . .

1.2 Quark charges. spins and masses . . . . . . . . . . . . . . . . . 3

1.3 Boson charges, spins, masses and interaction mediated . . . . . . . . 3

$1.4 E_{6} Z^{\prime}$ couplings . . . . . . . . . . . . . . . 12

2.1 Average store parameters . . . . . . . . . . . . . . . . . . . . . . . . . . . .

2.2 Summary of the CDF calorimeters . . . . . . . . . . . 26

3.1 Trigger requirements . . . . . . . . . . . . . . 35

4.1 Energy scale factors . . . . . . . . . . . . . . . . . . . . . 41

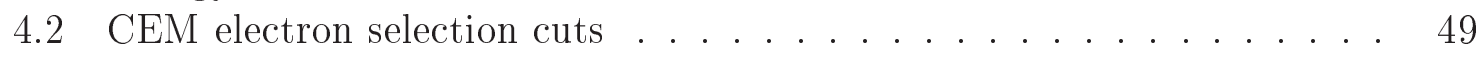

4.3 PEM electron selection cuts . . . . . . . . . . . . . . . 56

5.1 CEM selection cuts for the efficiency samples . . . . . . . . . . . . . 59

5.2 PEM selection cuts for the efficiency samples . . . . . . . . . . 59

5.3 Number of events and estimated background in each efficiency category 60

5.4 CEM N-1 identification efficiencies at the $Z^{0}$ pole $\ldots . . . . . . .60$

$5.5 \quad$ PEM N-1 identification efficiencies at the $Z^{0}$ pole . . . . . . . . . . . . 62

5.6 Total efficiencies and scale factors at the $Z^{0}$ pole . . . . . . . . . . 62

5.7 CEM efficiencies and scale factors for various mass windows . . . . . 64

5.8 PEM efficiencies and scale factors for various mass windows . . . . . 64

5.9 The measured $Z^{0} \rightarrow e^{+} e^{-}$cross-section in the range of $66-116 \mathrm{GeV} / c^{2} \quad 68$

6.1 Number of jet background events estimated in data . . . . . . . . . 70

6.2 Number of observed central-central events in each isolation region . . . 72

6.3 Number of observed central-plug events in each isolation region . . . 73

6.4 Number of MC predicted Drell-Yan CC events in each isolation region . $\quad 73$

6.5 Number of MC predicted Drell-Yan CP events in each isolation region. 73

6.6 The iet bkg. estimate for the $\mathrm{CC}$ channel for various region boundaries 74

6.7 The jet bkg. estimate for the $\mathrm{CP}$ channel for various region boundaries $\quad 75$

6.8 Uncertainties on the number of jet background events . . . . . . . . 75

6.9 The number of events with $S_{M E T}>2$ in the bkg isol $E_{T}^{\text {corr }}$ regions . . . 77

6.10 The loose selection cuts used for selecting an electron-like jet sample. . $\quad 78$

6.11 Theoretical cross-sections and expectations for the EWK and $\gamma \gamma$ bkgs. $\quad 83$ 


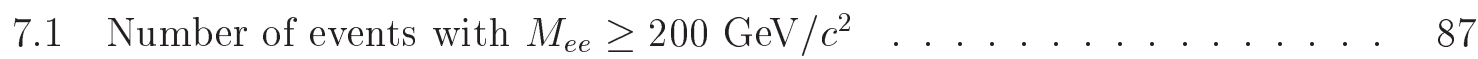

7.2 Summary of the systematic uncertainties affecting each channel . . . . 89

7.3 The percentage of pseudo-expts. observing a given p-value or lower . . 95

$7.4 Z^{\prime}$ mass limits . . . . . . . . . . . . . . . . . 103

7.5 Previous $Z^{\prime}$ mass limits $\ldots \ldots \ldots \ldots \ldots \ldots$

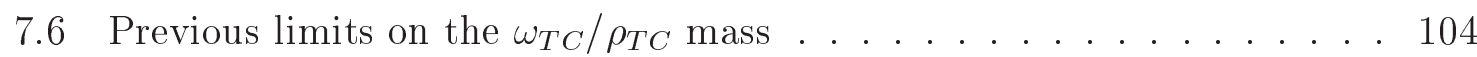

7.7 Randall-Sundrum graviton mass limits . . . . . . . . . . . . . 107

7.8 Previous Randall-Sundrum graviton mass limits . . . . . . . . . . . . 107

7.9 Svstematic uncertainties affecting the $e^{+} e^{-}$and $\gamma \gamma$ channels ..... . 110

7.10 Randall-Sundrum graviton mass limits for the $e^{+} e^{-}$and $\gamma \gamma$ channels . . 110

A.1 Central electron selection cuts used in previous CDF analvses . . . . . 116

A.2 Plug electron selection cuts used in previous CDF analyses . . . . . . . 117

A.3 Comparison of the new and the old very high- $p_{T}$ cuts . . . . . . . 117 


\section{Chapter 1}

\section{Introduction}

For thousands of years, humankind has struggled to answer the ultimate question of how the world around us functions. In this quest, several models have been proposed to explain or at least classify the interactions of the world around us. From the simple theory of the ancient Greeks dividing all matter into four elements; earth, water, fire and wind to our most complete model, the Standard Model of Particle Physics (SM), humankind has continually tested each model to determine where it breaks down. So far the Standard Model, described in section 1.1, has withstood every experimental test and currently all experimental measurements are consistent with it 1 . Still the Standard Model has several shortcomings, both aesthetic and physical, which lead physicists to believe that it is not the final answer but merely an empirically derived approximation to the behaviour of some more complete theory at our energy scale, much like Newtonian Mechanics is an approximation to Special Relativity at the speeds in the everyday world.

This analysis is a search for whatever new physics may lie beyond the Standard Model that produces a narrow high mass resonance decaying to two electron. As As there are many extensions/replacements for the Standard Model, this analysis will perform a model independent inclusive search for a high mass resonance decaying to two electrons. In order to be able to constrain the plethora of new physics models, limits on the cross-section times branching ratio to two electrons for spin-1 and spin-2 particles will then be calculated. In addition, specific limits will be placed on some popular models.

\subsection{The Standard Model}

The Standard Model is currently the most complete description of how fundamental particles interact. It has withstood every test so far and currently all experimental

\footnotetext{
${ }^{1}$ Recent observations which show neutrinos are not all massless [1] are inconsistent with the SM in which all neutrinos are massless. However the neutrino mass can still be incorporated by some minimal extensions to the SM.

${ }^{2}$ The term 'electron' is used to refer to both electrons and positrons throughout this thesis.
} 
results are consistent with it, although it has to be extended to account for neutrino oscillations. Formally the Standard Model is a quantum field theory which is invariant under the local gauge transformations $S U(3)_{C} \otimes S U(2)_{L} \otimes U(1)_{Y}$. The three gauge symmetries give rise to the three fundamental forces in the Standard Model; the strong force, the weak force and the electromagnetic force. The fourth force, gravity is not included in the Standard Model as, to date, the gravitational force has not been successfully quantised. However the gravitational force is not sufficiently strong at the scales of current collider energies for its effects to be observed. The strong force is described by the theory of Quantum Chromodynamics (QCD) [2] while electromagnetic and weak forces have been unified into a single theoretical framework, the GlashowSalam-Weinberg Model [3]. The strength of each force is not constant and depends on the $Q^{2}$ of the interaction. $Q^{2}$ is defined as $q^{2}$ for s-channel processes and as $-q^{2}$ for t-channel processes, where $q$ is the four-momentum of the probing particle.

\subsubsection{Particles of the Standard Model}

Particles in quantum field theory can be classed as either fermions or bosons. Fermions are particles which obey the Pauli Exclusion Principle and, as a consequence, have a $\frac{1}{2}$ integer spin, while bosons do not obey the Pauli Exclusion Principle and, as a consequence, have an integer spin. To date, no fundamental particle with a spin other than $\frac{1}{2}$ or 1 has been discovered, although the Higgs boson, which has a spin of 0 , is postulated as part of the Standard Model. Fermions comprise what is commonly considered matter while bosons mediate the interactions between the particles. There are twelve fermions plus their antiparticles in the Standard Model, organised into three generations, with each generation seemingly differing from the others only by the masses of the particles. Each generation consists of two quarks and two leptons plus their antiparticles. The quarks differ from the leptons by carrying colour charge, in addition to electric and weak isospin charges. Leptons are split into two groups: charged leptons and neutrinos. The charged leptons have a charge of -1 , the neutrinos have zero change, the up-type quarks have a charge of $+2 / 3$ and the down-type quarks have a charge of $-1 / 3$. The lepton and quark properties are summarised in tables 1.1 and 1.2 respectively. There are twelve spin-1 vector bosons in the Standard Model: the photon $(\gamma)$, the weak bosons $\left(W^{ \pm}, Z^{0}\right)$ and the eight gluons $(\mathrm{g})$. The photon mediates the electromagnetic interactions, the $W^{ \pm}$and $Z^{0}$ bosons mediate the weak interactions and the gluons mediate the strong interactions. Additionally there is one scalar boson, the Higgs boson, which generates the particles masses. However, to date, the existence of the Higgs boson has not been experimentally confirmed. The properties of the bosons in the Standard Model are summarised in table 1.3 .

\subsubsection{Standard Model Interactions}

The Standard Model is a gauge quantum field theory described by the symmetry group $S U(3)_{C} \otimes S U(2)_{L} \otimes U(1)_{Y}$. The $S U(3)_{C}$ symmetry group describes the interactions 


\begin{tabular}{|c|c|c|c|c|}
\hline Generation & Lepton & Charge & Spin & Rest Mass $\left(\mathrm{MeV} / c^{2}\right)$ \\
\hline \hline \multirow{2}{*}{ I } & electron $\left(e^{-}\right)$ & -1 & $1 / 2$ & 0.511 \\
& electron neutrino $\left(\nu_{e}\right)$ & 0 & $1 / 2$ & $<0.002$ at $95 \% \mathrm{CL}$ \\
\hline \multirow{2}{*}{ II } & muon $\left(\mu^{-}\right)$ & -1 & $1 / 2$ & 105.7 \\
& muon neutrino $\left(\nu_{\mu}\right)$ & 0 & $1 / 2$ & $<0.19$ at 90\% CL \\
\hline \multirow{2}{*}{ III } & tau $\left(\tau^{-}\right)$ & -1 & $1 / 2$ & $1776.99_{-0.26}^{+0.29}$ \\
& tau neutrino $\left(\nu_{\tau}\right)$ & 0 & $1 / 2$ & $<18.2$ at $95 \% \mathrm{CL}$ \\
\hline
\end{tabular}

Table 1.1: Lepton charges, spins and masses. All values are taken from [4].

\begin{tabular}{|c|c|c|c|c|}
\hline Generation & Quark & Charge & Spin & Rest Mass $\left(\mathrm{GeV} / c^{2}\right)$ \\
\hline \hline \multirow{2}{*}{ I } & up (u) & $+2 / 3$ & $1 / 2$ & $0.0015-0.0030$ \\
& down (d) & $-1 / 3$ & $1 / 2$ & $0.003-0.007$ \\
\hline \multirow{2}{*}{ II } & charm (c) & $+2 / 3$ & $1 / 2$ & $1.25 \pm 0.09$ \\
& strange (s) & $-1 / 3$ & $1 / 2$ & $0.095 \pm 0.025$ \\
\hline \multirow{2}{*}{ III } & top (t) & $+2 / 3$ & $1 / 2$ & $174.2 \pm 3.3$ \\
& bottom (b) & $-1 / 3$ & $1 / 2$ & $4.20 \pm 0.07$ \\
\hline
\end{tabular}

Table 1.2: Quark charges, spins and masses. All values are taken from [4].

\begin{tabular}{|c|c|c|c|c|}
\hline Boson & Charge & Spin & Rest Mass $\left(\mathrm{GeV} / c^{2}\right)$ & Interaction \\
\hline photon $(\gamma)$ & 0 & 1 & 0 & Electromagnetism \\
$W^{ \pm}$ & \pm 1 & 1 & $80.403 \pm 0.029$, & Weak \\
$Z^{0}$ & 0 & 1 & $91.1876 \pm 0.0021$ & Strong \\
gluon $(\mathrm{g})$ & 0 & 1 & 0 & \\
Higgs $(\mathrm{H})$ & 0 & 0 & $114.4<M_{H}<203$ at $95 \% \mathrm{CL}$ & \\
\hline
\end{tabular}

Table 1.3: Boson charges, spins, masses and interaction mediated. Note that the Higgs Boson has not been experimentally observed. All values are taken from [4]. 
of the strong force while the $S U(2)_{L} \otimes U(1)_{Y}$ symmetry group describes the unified interactions of the electroweak group. The theory is calculated through the use of perturbation theory which separates the terms in the matrix element by the order of the coupling constant, with each gauge boson interaction in a term adding a power to the coupling constant. This can be represented diagrammatically using Feynman diagrams. Perturbation theory makes use of the fact that the coupling constants are small so higher order terms will become vanishingly small. For the electroweak interactions this is valid but the validity of this for the strong interactions depends on the scale of the interaction.

\section{Electroweak Theory}

The electroweak theory describes the electromagnetic and weak forces in the same theoretical framework. The electroweak sector is described by the $S U(2)_{L} \otimes U(1)_{Y}$ symmetry group, from which the $U(1)_{E M}$ symmetry of electromagnetism is obtained. The $U(1)_{Y}$ group represents the weak hypercharge gauge symmetry, while the $S U(2)_{L}$ group represents the weak isospin gauge symmetry. The $L$ in the $S U(2)_{L}$ indicates that it is only left-handed fermions which transform under $S U(2)_{L}$. The $S U(2)_{L}$ gauge symmetry gives rise to a triplet of fields, $W^{i}$, associated with the weak isospin, while the $U(1)_{Y}$ gauge symmetry gives rise to a single field, $B$, associated with the weak hypercharge. The physical states of these fields are related to the fundamental fields by

$$
\begin{gathered}
W_{\mu}^{ \pm}=\frac{1}{\sqrt{2}}\left(W_{\mu}^{1} \mp i W_{\mu}^{2}\right) \\
\left(\begin{array}{c}
Z_{\mu} \\
A_{\mu}
\end{array}\right)=\left(\begin{array}{cc}
\cos \theta_{W} & -\sin \theta_{W} \\
\sin \theta_{W} & \cos \theta_{W}
\end{array}\right)\left(\begin{array}{c}
W_{\mu}^{3} \\
B_{\mu}
\end{array}\right)
\end{gathered}
$$

where $\theta_{W}$ is the weak mixing angle.

The local gauge invariance requires that all the fermions and bosons of the theory are massless, which is in contrast to experimental observation. In the Standard Model, the masses are generated using the Higgs mechanism. The Higgs mechanism introduces a $S U(2)$ doublet of complex scalar fields with a non-zero vacuum expectation value. The breaking of the symmetry gives rise to three massless scalar Goldstone bosons and one massive real scalar boson, the Higgs boson. Through interactions with the Higgs field, the $W^{ \pm}$and $Z^{0}$ bosons acquire masses in the ratio

$$
\frac{M_{W}}{M_{Z}}=\cos \theta_{W}
$$

and the three Goldstone bosons form their resulting longitudinal modes. The fermions can also acquire masses through interacting with the Higgs field, and this depends on the arbitrary coupling of the fermion field to the Higgs field. The Higgs boson has so far not been observed experimentally but is predicted to have a mass less than $203 \mathrm{GeV} / c^{2}$ from precision electroweak fits and is excluded at masses less than $114 \mathrm{GeV} / c^{2}$ from direct searches at LEP [4]. 


\section{Quantum Chromodynamics}

The strong force results from the $S U(3)_{C}$ gauge symmetry of the Standard Model and is described by the theory of Quantum Chromodynamics (QCD). There are eight generators of $S U(3)_{C}$, giving rise to eight gluon fields. These generators do not commute and this non-Abelian nature results in gluon-gluon interactions. The self-interactions of the gluon field gives rise to the most striking features of the model, asymptotic freedom and confinement. Confinement is the property of the theory that requires that all observed states in nature have zero net colour charge. Quarks and gluons carry a net colour charge and therefore exist in bound states known as hadrons. This is consistent with experiment as no free quark or gluon has been observed to date.

Related is the concept of asymptotic freedom where at small distance scales and thus high $Q^{2}\left(Q^{2}\right.$ above $\left.\approx 4 \mathrm{GeV}^{2} / c^{4}\right)$, the gluons and quarks behave as if they were free particles. This allows the high- $p_{T}$ behaviour of quarks and gluons to be calculated using perturbation theory, although the coupling constant is still large enough to make next-to-leading order and next-to-next-to-leading order terms significant. At low $Q^{2}$ and thus large distance scales, the coupling constant becomes very large and perturbation theory is no longer valid to calculate the solution. Some low $Q^{2}$ properties can be calculated numerically using lattice QCD. However as discussed below, phenomenological models are used in analyses of the type described here. In high energy collisions, quarks and gluons interact as effectively free particles. After the collision, the quarks and gluons become increasingly separated as they move away from the collision point. The strength of the strong force between them becomes of progressively higher strength as the distance increases. This leads to a process known as hadronisation, where the free quark or gluon combines with new coloured objects produced from the vacuum to create colour neutral bound states. This process is inherently non-perturbative and, as such, is very difficult to calculate. The process results in the production of a 'jet' of hadrons travelling in the direction of the original quark or gluon, which can then be detected experimentally. The hadronisation process, by which partons 3 produce jets of hadrons with limited $p_{T}$ with respect to the initial parton direction is described by phenomenological fragmentation models, such as PYTHIA [5] and HERWIG [6].

QCD is important in hadron colliders as it describes the parton content of the colliding hadrons, with the constituent partons being the objects that actually interact in the collision. In the parton model, the proton is made up of three constituent quarks, two up and one down (uud). In QCD, the radiation of gluons by the quarks leads to a rich structure of the proton which varies as a function of $Q^{2}$. For example, at low $Q^{2}$, the three quarks of the parton model carry approximately $50 \%$ of the proton momentum, with almost all of the rest of the momentum carried by gluons, while at higher $Q^{2}$ the sea quark-antiquark pairs become more important. Therefore to be able to correctly describe the initial state of a $p \bar{p}$ interaction requires a precise knowledge of the relative content and the momenta distributions of partons making up the proton as a function of $Q^{2}$. This is described by parton distribution functions (PDFs), which are obtained

\footnotetext{
${ }^{3} \mathrm{~A}$ parton is a generic term for any constituent of a hadron and refers to both quarks and gluons.
} 
from global QCD fits to many sources of experimental data, with an important fraction coming from studying $e^{ \pm} p$ collisions at the HERA collider at DESY. The actual parton distributions cannot currently be predicted theoretically but their evolution with $Q^{2}$ is calculable using perturbative QCD in the form of the DGLAP equations [7]. Therefore a given shape for the parton distributions for some starting value of $Q^{2}$ is evolved to different values of $Q^{2}$ and the resulting predictions are compared to experimental data, varying the starting shape to get the best match.

\subsubsection{Di-Electron Production in the Standard Model}

\section{The Drell-Yan Process}

The Drell-Yan process is the name given to inclusive production of opposite sign dilepton pairs in hadron-hadron collisions, written as $h_{i}+h_{j} \rightarrow l^{+} l^{-}+X$ where $h_{i}$ and $h_{j}$ are the colliding hadrons, $l^{ \pm}$are the leptons produced directly by the mediating particle and $X$ refers to the remaining particles produced in the process. It is named the Drell-Yan process in honour of S. D. Drell and T. M. Yan who proposed the first successful description of lepton pair production in hadron collisions in the context of the parton model [8].

In the Standard Model the Drell-Yan process is mediated by either a virtual photon $\left(\gamma^{*}\right)$ or $Z^{0}$ boson. Figure 1.1 shows this process at leading order (LO). Next-to-leading order (NLO) diagrams for this this process are shown in figure 1.2. The contributions from these diagrams is significant and leads to an increase in cross-section of about $30 \%$ at TeV scales [9]. To account for NLO processes a multiplicative k-factor of 1.3 is applied to all leading order Drell-Yan cross-section predictions made during the course of this analysis. This is in part chosen to be consistent with past Drell-Yan resonance searches at CDF [10].

The Drell-Yan mass spectrum is an ideal place to search for a yet to be discovered particle, and in the past has lead to the discovery of the $Z^{0}$ boson at the UA1 and UA2 experiments at CERN [11]. Any new particle which mediates the Drell-Yan process will show up as a resonance at the mass of the particle, an example being the $Z^{0}$ resonance at $91.18 \mathrm{GeV} / c^{2}$. The presence of the well measured $Z^{0}$ resonance in the Drell-Yan mass spectrum also provides a useful energy calibration point and selection efficiency cross-check, further enhancing its usefulness as a new physics search tool. The DrellYan mass spectrum also provides a useful test of QCD, since it is a relatively clean process which probes the $q \bar{q}$ distributions of the colliding hadrons at high momenta.

\section{Non Drell-Yan Di-Electron Production in the Standard Model}

The Drell-Yan process is not the only way to produce a final state of two electrons in an event at a hadron collider. $W^{ \pm}$bosons can decay to a charged lepton and a neutrino, so any process in which two $W$ bosons are produced can potentially produce a final state which includes two electrons. Also $Z^{0}$ bosons can be produced in association with $W^{ \pm}$bosons due to the non-Abelian nature of the $S U(2)$ group, and these events 


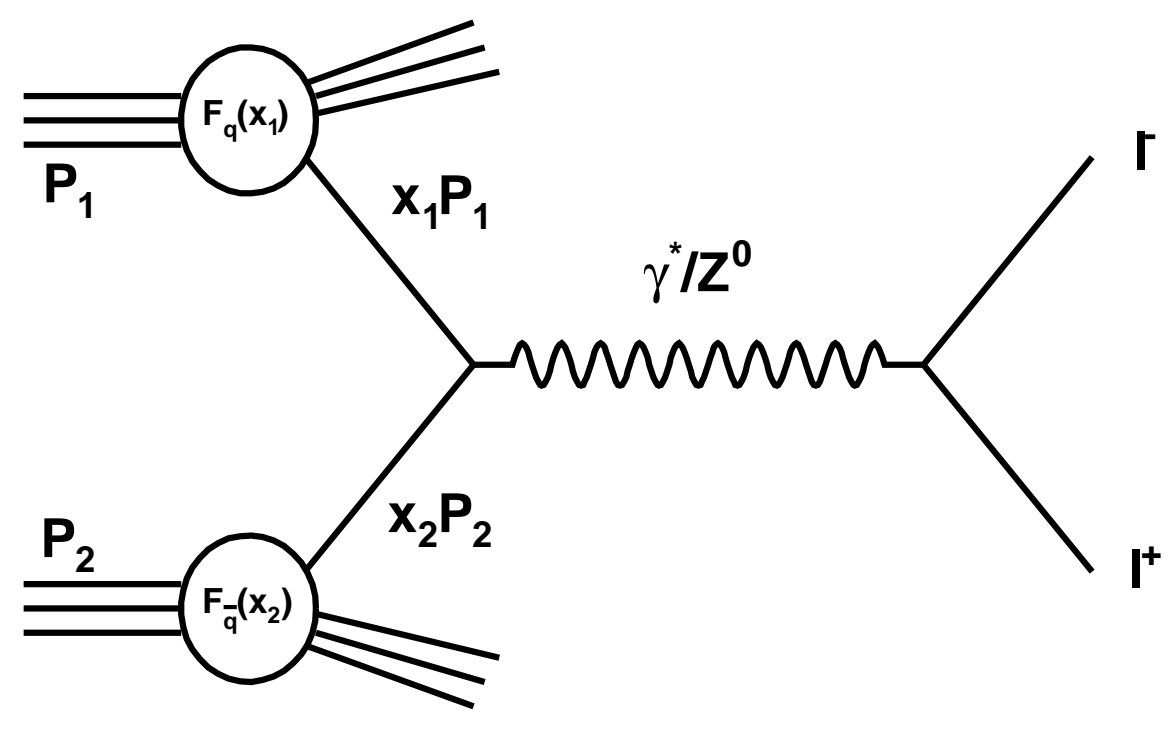

Figure 1.1: The Standard Model Drell-Yan process at LO in $p \bar{p}$ collisions. The incoming proton and antiproton have momenta $P_{1}$ and $P_{2}$ respectively, of which a fraction $x_{1}$ and $x_{2}$ is carried by the interacting quarks.
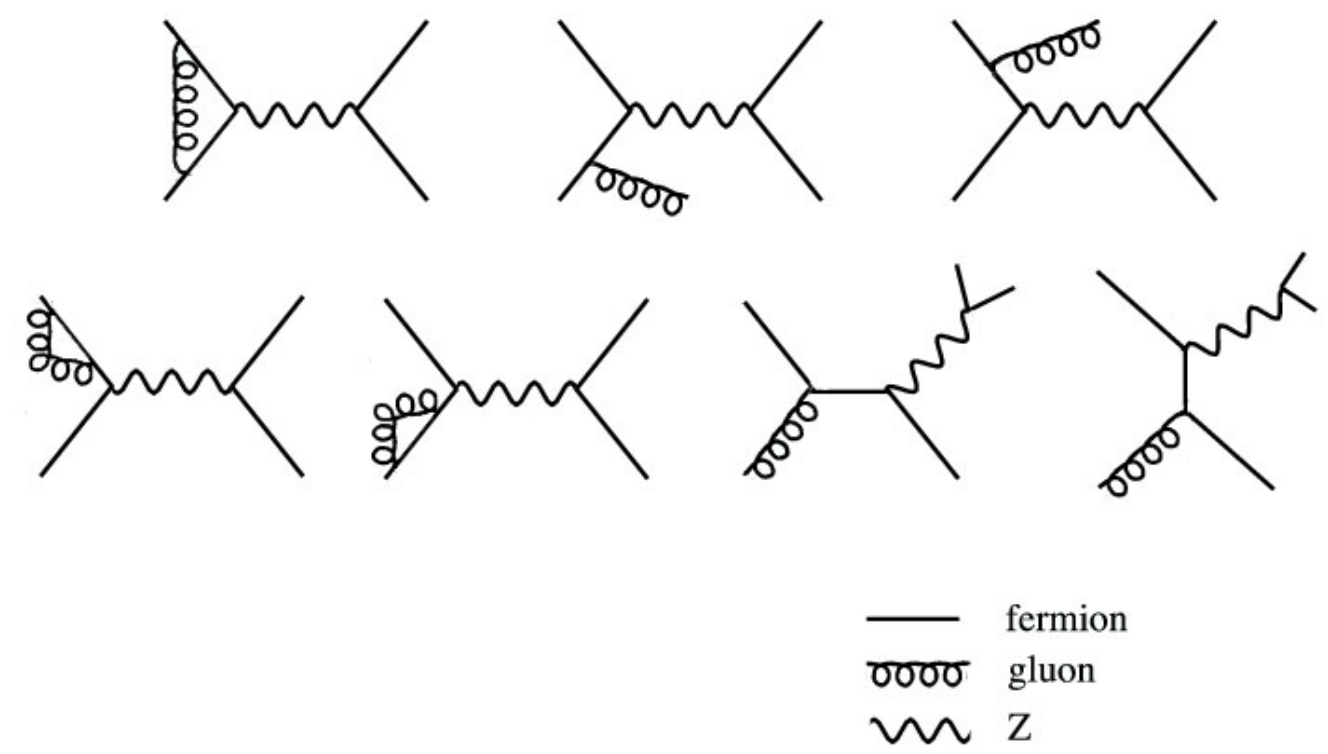

Figure 1.2: Next-to-leading order contributions to the SM Drell-Yan Process. 

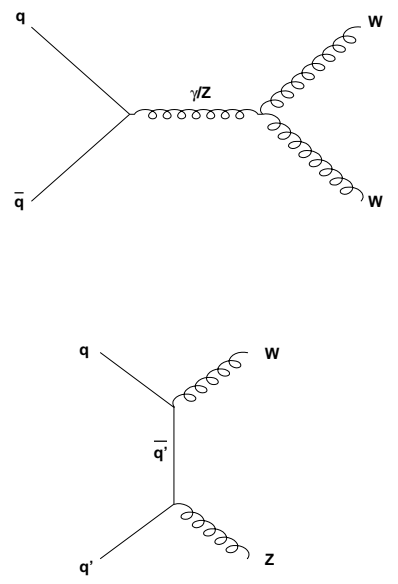
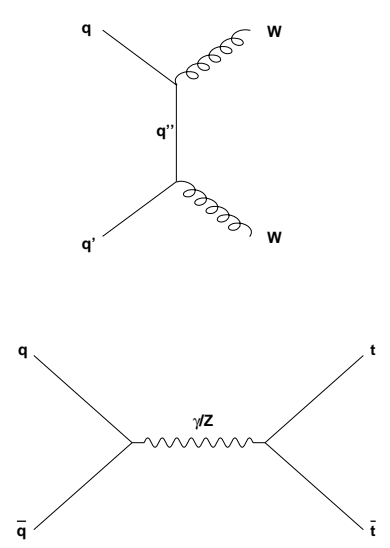
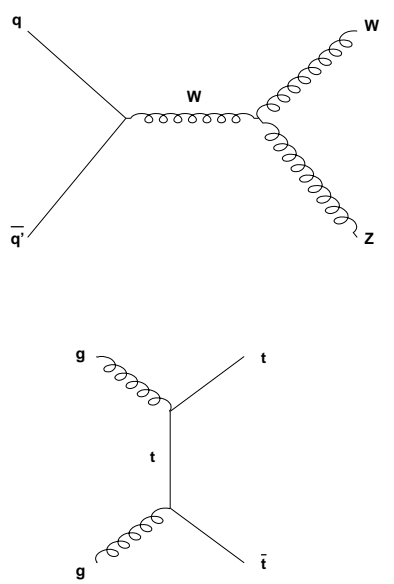

Figure 1.3: Non Drell-Yan processes which can produce two leptons in the final state

can also have a final state which includes two electrons. Finally the Drell-Yan process with final state $\tau$ leptons can result in lighter lepton generation pairs being produced. The dominant sources of events other than Drell-Yan which include a final state of two leptons are

1. $W^{+} W^{-} \rightarrow l^{+} l^{-} \nu_{l} \overline{\nu_{l}}$

2. $W^{ \pm} Z$, where $Z \rightarrow l^{+} l^{-}$

3. $Z / \gamma^{*} \rightarrow \tau^{+} \tau^{-} \rightarrow l^{+} l^{-} \nu_{\tau} \nu_{l} \bar{\nu}_{\tau} \bar{\nu}_{l}$

4. $t \bar{t} \rightarrow l^{+} l^{-} \nu_{l} \overline{\nu_{l}} b \bar{b}$

and the most significant Feynman diagrams for these processes are shown in figure 1.3. However, as described in section 6.4, the cross-sections for these final states are significantly smaller than for the Drell-Yan process.

\subsubsection{Problems with the Standard Model}

While the Standard Model has been remarkably successful, the model is not without its problems. Some of these problems are aesthetic, some more serious. It is a general feeling of physicists that nature is simple and introducing too many arbitrary values with little relation to each other is an indication that something is wrong with the theory. While this is not a scientific reason to reject a theory, it does lead to scepticism about whether the theory is truly fundamental. The main problems of the Standard Model are listed below. 


\section{Gravity}

The most glaring problem with the current Standard Model is that a description of gravity does not exist. This is in part due to gravity being extremely weak and thus so far undetectable in collider experiments. The resulting lack of any positive experimental data from particle colliders has hindered the development of a successful quantum theory of gravity. However the Standard Model cannot be a complete theory of everything until gravity is included. Many theories of gravity have been developed with the most popular being string theory, which abandons treating particles as point-like objects and instead treats them as the vibration modes of string in a 10 or 26 dimensional space [12].

\section{Grand Unification}

In the Standard Model there are three forces with the fourth force gravity not yet successfully included. It is thought that at some scale, known as the Grand Unification (GUT) scale, the three forces are unified into one fundamental force. Evidence for this is that the coupling constants of the three forces have a $Q^{2}$ dependence such that, at $Q \approx 10^{15} \mathrm{GeV}$, they come very close to all being equal. The fact that they do not exactly meet at the same point implies that perhaps some new physics occurs between here and the GUT scale, such as supersymmetry [13], which alters the scaling of the coupling constants with energy so that they all meet at the same point.

\section{The Hierarchy Problem}

The Standard Model has several scales within it, the electroweak scale of a TeV, the GUT scale, if it exists, of $\sim 10^{15} \mathrm{GeV}$ and the Planck scale of $10^{19} \mathrm{GeV}$. These scales are many orders of magnitude apart and this large hierarchy of scales has no explanation in the Standard Model. While this is an aesthetic problem, it is an indication that perhaps there is an underlying theory to the Standard Model that would explain this naturally. A popular solution to this problem is to introduce extra dimensions which allows all the scales of the Standard Model to be of the same order but appear vastly different in our $3+1$ dimensional world depending on how much the fundamental fields overlap with our brane.

\section{The Fine Tuning Problem}

In the Standard Model, the particle masses are generated via the Higgs mechanism although this has not been experimentally verified. This introduces a fundamental scalar, the Higgs boson, whose mass is quadratically unstable against radiative corrections. In a problem related to the hierarchy problem, in order to have a light Higgs boson mass of less than the Planck Mass, the bare Higgs boson mass must be fine tuned to one part in $10^{24}$ [14] to exactly cancel the radiated corrections. Again this 
is an aesthetic problem, but theories have been proposed such as supersymmetry and technicolour [15] which either solve or remove this problem in a natural way.

\section{The Baryon Asymmetry Problem}

One of the more fundamental problems of the Standard Model is the observed baryon asymmetry of the universe. Our universe is predominately made of matter and has little anti-matter, although both should have been produced equally in the Big Bang. $\mathrm{CP}$ violation gives an asymmetry between matter and anti-matter but the sources of $\mathrm{CP}$ violation in the Standard Model are not large enough to account for the observed asymmetry. This implies that there is an yet undiscovered CP violation mechanism outside of the Standard Model.

\section{The Dark Matter Problem}

Another fundamental problem of the Standard Model is that it offers no explanation for the dark matter problem [16]. The dark matter problem comes from astronomical observations that the rotation curves of galaxies are inconsistent with theoretical predictions unless there is a large amount of matter present additional to that visible in astronomical observations. This matter is know as 'dark matter' as it does not emit or reflect enough electromagnetic radiation to be detected directly. Dark matter accounts for about 20-30\% of the universe [4], with dark energy accounting for $\sim 70 \%$ and visible matter accounting for about 5\% [17]. While planets, dwarf stars, black holes and neutrinos account for some of the dark matter within the Standard Model framework, it is commonly suspected that dark matter consists of mostly of weakly interacting massive particles (WIMPs) which are currently not contained within the Standard Model. As such many extensions/replacements for the Standard Model also include a dark matter candidate, the most popular example being the lightest neutralino in supersymmetry. However the motivation for dark matter does assume that current gravitational theories are correct and it may be that a modification of current theories may be sufficient to explain the observed gravitational effects.

\subsection{New Physics Beyond The Standard Model}

There are many extensions/replacements available for the Standard Model, most of which are variations on a few themes. These themes include supersymmetry, string theory, grand unification, extra gauge symmetries and extra dimensions. Specific models which are explicitly searched for in this analysis are briefly described below.

\footnotetext{
${ }^{4}$ Dark energy is a hypothetical form of energy which permeates all of space, has a strong negative pressure and therefore has a similar effect to a force acting against gravity at large distance scales [17. However it has not been conclusively established that dark energy exists.
} 


\subsubsection{Additional $U(1)$ Gauge Symmetries}

Any additional gauge symmetry will manifest itself as a new massive neutral spin-1 boson, referred to as a $Z^{\prime}$, which can appear as a narrow resonance in the Drell-Yan mass spectrum. Various models predict additional $U(1)$ gauge symmetries, with most occurring from the breaking of the GUT symmetry group down to the Standard Model. The most popular choices for the GUT symmetry group are $S U(5), S O(10)$ and $E_{6}$. The $S U(5)$ group is the smallest group which contains the $S U(3)_{C} \otimes S U(2)_{L} \otimes U(1)_{Y}$ symmetry groups of the Standard Model, however it predicts $\sin ^{2} \theta_{W}=0.214 \pm 0.004$ [14] at the scale of the mass of the $W$ boson, while the experimental result is $\sin ^{2} \theta_{W}=$ $0.22306 \pm 0.00033$ [4]. It also predicts a proton lifetime of $\sim 10^{30}$ yr [14], while the experimental lower limit is $1.6 \times 10^{33} \mathrm{yr}$ [4]. Therefore the $S U(5)$ group alone is strongly disfavoured by experiment. The symmetry groups $S O(10)$ and $E_{6}$ both contain $S U(5)$ and therefore the Standard Model gauge symmetries and are not in contradiction with experimental results. The $E_{6}$ and $S O(10)$ symmetry groups are broken as follows

$$
\begin{gathered}
E_{6} \rightarrow S O(10) \otimes U(1)_{\psi} \\
S O(10) \rightarrow S U(5) \otimes U(1)_{\chi}
\end{gathered}
$$

and in both cases additional $U(1)$ gauge symmetries are left over, resulting in $Z^{\prime}$ bosons. The couplings of the $Z^{\prime}$ bosons to other particles is highly model dependent and no prediction is made on the masses, although they can be favoured to be of order $1 \mathrm{TeV}$ in superstring theories [18]. The $Z^{\prime}$ bosons of the $E_{6}$ gauge group particularly attract attention as the $E_{6}$ gauge group is strongly motivated by string theory [19]. The $E_{6}$ group gives two additional neutral spin-1 gauge bosons; the $Z_{\psi}^{\prime}$ resulting from the breakdown to $S O(10)$ and the $Z_{\chi}^{\prime}$ from the breakdown of $S O(10)$ to $S U(5)$. These new $Z^{\prime}$ bosons are related to the physical states via

$$
Z^{\prime}(\theta)=Z_{\psi}^{\prime} \cos \theta+Z_{\chi}^{\prime} \sin \theta
$$

where $\theta$ is the mixing angle. While the mixing angle can have any value, the values of 0 , $\pi / 2, \sin ^{-1} \sqrt{3 / 8}$ and $\sin ^{-1} \sqrt{5 / 8}$ correspond respectively to the canonical $E_{6} Z^{\prime}$ bosons: the $Z_{\psi}^{\prime}$, the $Z_{\chi}^{\prime}$, the $Z_{\eta}^{\prime}$ and the $Z_{I}^{\prime}$. The couplings for these $Z^{\prime}$ bosons to Standard Model particles can be found in [20] and are summarised in table 1.4. Another popular $Z^{\prime}$ is the sequential or SM-like $Z^{\prime}\left(Z_{S M}^{\prime}\right)$. This $Z^{\prime}$ couples identically to fermions as the SM $Z^{0}$ does and therefore has identical production and decay properties to the $Z^{0}$ once its higher mass is taken into account. Unlike the $E_{6} Z^{\prime}$ bosons, the $Z_{S M}^{\prime}$ boson is poorly motivated theoretically and is typically only used as a simple benchmark of analysis sensitivity.

\subsubsection{Technicolour}

Technicolour is an alternative to the Higgs mechanism used to generate the masses of Standard Model particles [15]. It does this by dynamically breaking the electroweak 


\begin{tabular}{|c|c|cc|cc|cc|cc|}
\hline \multirow{2}{*}{$E_{6} Z^{\prime}$} & \multirow{2}{*}{$\sin \theta$} & \multicolumn{2}{|c|}{$d$} & \multicolumn{2}{c|}{$u$} & \multicolumn{2}{|c|}{$e$} & \multicolumn{2}{c|}{$\nu_{e}$} \\
\cline { 3 - 9 } & & $\mathrm{V}$ & $\mathrm{A}$ & $\mathrm{V}$ & $\mathrm{A}$ & $\mathrm{V}$ & $\mathrm{A}$ & $\mathrm{V}$ & $\mathrm{A}$ \\
\hline \hline$Z_{\psi}^{\prime}$ & 0 & 0 & $\frac{\sqrt{10} s}{3}$ & 0 & $\frac{\sqrt{10} s}{3}$ & 0 & $\frac{\sqrt{10} s}{3}$ & $\frac{\sqrt{10} s}{6}$ & $\frac{\sqrt{10} s}{6}$ \\
$Z_{\chi}^{\prime}$ & 1 & $\frac{2 \sqrt{6} s}{3}$ & $-\frac{\sqrt{6} s}{3}$ & 0 & $\frac{\sqrt{6} s}{3}$ & $-\frac{2 \sqrt{6} s}{3}$ & $-\frac{\sqrt{6} s}{3}$ & $-\frac{\sqrt{6} s}{2}$ & $-\frac{\sqrt{6} s}{1}$ \\
$Z_{\eta}^{\prime}$ & $\sqrt{\frac{3}{8}}$ & $s$ & $\frac{s}{3}$ & 0 & $4 s$ & $-s$ & $\frac{s}{3}$ & $-\frac{s}{3}$ & $-\frac{s}{3}$ \\
$Z_{I}^{\prime}$ & $\sqrt{\frac{5}{8}}$ & $\frac{\sqrt{15} s}{3}$ & $-\frac{\sqrt{15} s}{3}$ & 0 & $-\frac{\sqrt{15} s}{3}$ & $-\frac{\sqrt{15} s}{3}$ & $-\frac{\sqrt{15} s}{3}$ & $-\frac{\sqrt{15} s}{3}$ & $-\frac{\sqrt{15} s}{3}$ \\
\hline
\end{tabular}

Table 1.4: Vector and axial couplings of the $E_{6} Z^{\prime}$ bosons to Standard Model particles. The couplings are identical for the different generations. The notation $s=\sin \theta_{W} \approx$ 0.23 is used.

symmetry using strong dynamics rather than spontaneously as the Higgs mechanism does. Technicolour is a QCD-like theory, which introduces a set of new particles, the technifermions. These new particles carry the technicolour charge and form bound states of pseudoscalar and vector mesons, analogously to QCD. The pseudoscalar mesons, the technipions $\left(\pi_{T}\right)$, are the pseudo-Goldstone bosons of the theory, three of which are then used to make up the longitudinal modes of the $W^{ \pm}$and $Z^{0}$ bosons. While technicolour offers some advantages over the Higgs model, the most notable being having no fundamental scalars present in the theory and therefore removing the need for fine tuning, it does have several issues with the most notable being flavour changing neutral currents and its difficultly in producing a top mass of order $175 \mathrm{TeV} / c^{2}$ [21]. These issues have led to its relative disfavour among the physics community. Improved technicolour theories avoid these problems by having a 'walking' coupling constant, as opposed to a 'running' coupling constant like QCD, and by introducing a new interaction, TopColour, which gives the top quark its mass [21]. The model used in this analysis is the Technicolour 'Straw Man's' Model (TCSM) [22, which describes the collider phenomenology for the lightest colour singlet of technihadrons. In this model, the members of the lightest technifermion doublet $\left(T_{U}, T_{D}\right)$ are colour singlets, and they transform under technicolour $S U\left(N_{T C}\right)$ and have electric charges $Q_{U}$ and $Q_{D}=Q_{U}-1$. They form bound states of pseudoscalar and vector mesons. The pseudoscalar mesons consist of a spin- 0 isotriplet $\Pi_{T C}^{ \pm, 0}$ and an isosinglet $\Pi_{T C}^{0^{\prime}}$. Similarly, the vector mesons consist of a spin-1 isotriplet $\rho_{T C}^{ \pm, 0}$ and an isosinglet $\omega_{T C}$, with the two neutral vector mesons being able to mediate the Drell-Yan process. The main parameters of the model are

$$
\begin{aligned}
Q_{U} & =Q_{D}+1=4 / 3 \\
\sin \chi & =1 / 3 \\
F_{T} & =82 \mathrm{GeV} \\
N_{T C} & =4 \\
M_{V} & =M_{A}=200 \mathrm{GeV} \\
M_{\rho} & \approx M_{\omega}=M_{\pi}+100 \mathrm{GeV}
\end{aligned}
$$


where $F_{T}$ is the Technicolour decay constant, $\chi$ is the mixing angle between the technipion interaction and mass eigenstates, $M_{V}$ and $M_{A}$ are the vector and axial mass parameters which effect the decay of the mesons to transverse gauge bosons and technipions and $M_{\rho}, M_{\omega}$ and $M_{\pi}$ are the masses of the technimesons. The $\omega_{T C}$ and $\rho_{T C}^{0}$ can be produced in $p \bar{p}$ collisions and can subsequently decay to di-electron pairs, producing a narrow spin-1 resonance in the Drell-Yan mass spectrum which, in this analysis, is indistinguishable from a resonance produced by a $Z^{\prime}$ boson of the same mass.

\subsubsection{Warped Extra Dimensions}

In an attempt to solve the hierarchy problem, Randall and Sundrum proposed an additional dimension with a non-factorisable geometry 23. This simply means that the observables in the 3 spacial dimensions also depend on the position in the additional dimension. In most extra-dimensional theories, gravity can appear weaker than its true strength on our brane due to it spreading itself out into the other dimensions, with the relation to the effective reduced Planck scale, $\bar{M}_{p l}=M_{p l} / \sqrt{8 \pi}$, and the true Planck scale $\left(M_{D}\right)$ given as follows

$$
\bar{M}_{p l}^{2}=M_{D}^{n+2} V_{n}
$$

where $n$ is the number of additional dimensions and $V_{n}$ is the volume of the compact space. In previous extra-dimensional theories [24], the hierarchy between the Planck scale and the weak scale is eliminated by having many extra dimensions and/or making the volume of the extra dimensions large. Randall and Sundrum instead introduce two branes with opposite tension in a 5-dimensional space based on a slice of $A d S_{5}$ space with a non-factorisable metric

$$
d s^{2}=e^{-2 k r_{c} \phi} \eta_{\mu \nu} d x^{\mu} d x^{\nu}+r_{c}^{2} d \phi^{2}
$$

where $k$ is a scale of order the Planck scale, $x^{\mu}$ are the coordinates for the four dimensional space, $\phi$ is the coordinate for the extra dimensions and has the range $0 \leq \phi \leq \pi$ and $r_{c}$ is the compactification radius. The two branes are located at $\phi=0$ and $\phi=\pi$ and are referred to the UV and IR branes respectively. The major consequences of the non-factorisable metric of the 5-dimensional space is that the 4-dimensional metric depends on the position in the extra dimension. This leads to the exponential warp factor in the 4-dimensional metric which reduces the true Planck scale to the effective Planck scale observed in our 4-dimensional world. The relationship between the effective Planck scale and the true Planck scales becomes

$$
\bar{M}_{p l}^{2}=\frac{M_{D}^{3}}{k}\left[1-e^{-2 k r_{c} \pi}\right] .
$$

With the model assumption of large $k r_{c}$, this implies that $M_{p l}$ only depends weakly on $k r_{c}$ and is within a couple orders of magnitude of $M_{D}$ which is not enough solve the 
hierarchy problem. However the observed masses on the IR brane are related to the masses on the UV brane by

$$
m_{I R}=m_{U V} e^{-k r_{c} \pi}
$$

This allows all masses in the higher dimension theory to be of order $\bar{M}_{p l}$ yet appear as order $\mathrm{TeV}$ when observed in the physical universe. The situation can also be reversed and the TeV scale taken as the fundamental scale. In this scenario, the reason gravity appears weak is that it is concentrated at the other brane and has an exponentially small overlap with the SM brane due to the non-trivial geometry of the extra dimension and not that it is diluted over a much larger volume as in previous extra dimensional models. Regardless of the interpretation, the size of the extra dimension is allowed to be small, and thus limits on models which predict large extra dimensions from LEP and supernova observations do not apply.

In the simplest model, which is the only model considered in this analysis, the experimental signature is a series of spin-2 resonances, referred to as Randall-Sundrum $(\mathrm{RS})$ gravitons, which decay to pairs of all Standard Model particles. The resonance masses begin at order $\mathrm{TeV}$ and are given by

$$
m_{n}=k x_{n} e^{-k r_{c} \pi}
$$

where $m_{n}$ is the mass of the $n^{\text {th }}$ resonance and $x_{n}$ is the $n^{\text {th }}$ root of the Bessel function of order 1 . In this analysis, only the first resonance will be considered and this resonance will be referred to as the graviton for simplicity. The phenomenology of the model depends only on two parameters, the mass of the graviton $\left(M_{G}\right)$ and the ratio $k / \bar{M}_{p l}$ which enters quadratically in the width of the graviton. The mass of the graviton is of order $\mathrm{TeV}$, while the favoured range for $k / \bar{M}_{p l}$ is $0.01-0.1$ [25, 26].

\subsection{Analysis Strategy}

The aim of this analysis is to search for a narrow 5 di-electron resonance in a specified search range with no other assumptions made about the spin, couplings or mass of the particle. The search range is defined to be the invariant mass range of $150 \mathrm{GeV} / c^{2}$ to $950 \mathrm{GeV} / c^{2}$. There are also kinematic and geometrical constraints on the produced electrons which are defined later. After the search, the resulting mass spectrum will also be interpreted to place limits on generic spin- 1 and spin- 2 particles and on the specific models detailed in section 1.2. As this is a search for new physics, the term signal will refer to a new physics process and the Standard Model Drell-Yan process will be considered an irreducible background to this.

In order to minimise the possibility of accidental bias in the analysis and therefore to produce a robust statistically meaningfully result, the analysis is performed in a

\footnotetext{
${ }^{5}$ In the context of this analysis narrow means that the resolution of the detector dominates the width
} 
'blind' manner. This means that all of the arbitrary choices that are made during the course of the analysis, such as the signal search range, the selection cuts and the interpretation method, are made without reference to the signal region. This is implemented by automatically discarding any events selected by the selection criteria with $M_{e e}$ greater than $150 \mathrm{GeV} / c^{2}$ until the analysis is finalised. When the analysis is finalised, the data are 'unblinded' by no longer discarding these events.

Periodically the data are unblinded when there is sufficient data to publish a result. The first $200 \mathrm{pb}^{-1}$ of data had already been published at the start of this analysis [10] and therefore this analysis was cross-checked against that published result to check for errors. The analysis was subsequently unblinded for the next running period of data, which corresponds to a total luminosity of $368 \mathrm{pb}^{-1}$, and subsequently published [27]. Any data which have been previously unblinded are not used to re-tune the analysis cuts or otherwise bias the analysis, however unblinded data are used to check that the background estimates are sensible. This is achieved by constructing background dominated samples and checking if the method used to estimate the background can correctly predict the mass spectrum of these samples. As these samples are background dominated, the presence of a new physics signal cannot significantly influence this crosscheck.

Like any result in science, the author built upon work of others. The general analysis used the work done previously in this channel [10] as a starting point although very little was not extensively re-optimised or improved. For example, as discused in appendix $\mathrm{A}$, the selection cuts were re-optimised and bare little similarities. The methods for estimating the jet background in chapter 6 were improved and control samples were introduced to verify the background predictions. The limit setting technique used in chapter 7 is the same as that used by the previous analysis but the software package used to perform it was written solely by the author. The author also lead the combination procedure with the di-photon analysis discussed at the end of chapter 7 , which was made possible by the limit setting package the author had written. The author also introduced a new method not previously used in new physics searches at CDF to search for an excess over the Standard Model prediction and this is also described in chapter 7. Additionally, as in all large collaborations, a set of tools and methods exist for common use. Examples include Monte Carlo generators tuned to the experiment, ntuple formats, common efficiency measurements and data handling software. For example, all the data samples and Monte Carlo samples used in this analysis and discused in chapter 3 were generated centrally with the exception of the high mass Drell-Yan, $Z^{\prime}$ and RS Graviton Monte Carlo samples which were generated using the tools of others. The analysis started with the CDF standard ntuples, some which of were made by the author as a contribution to a large central effort using software which author contributed to improving, but did not develop. After that, almost all analysis code was written by the author, making heavy use of the ROOT libraries [28]. Some of the methods that are used in this analysis are the standard way of doing a task at CDF, such as the method used to measure the electron identification efficiency in chapter 5, and some parameters measured by other analyses are used to save time, 
such as the ISR uncertainty on the acceptance also described in chapter 5. In addition, the calibration of the electron energy and track $p_{T}$ are all done centrally although for the case of plug electrons, the author was responsible for working out the best way to obtain the energy and how best to calibrate it. All the methods and techniques that are standard methods and techniques at CDF are clearly referenced and all work that is not the authors is also clearly referenced.

The CDF collaboration uses internal and public notes to document analyses and procedures used at CDF. Internal notes are restricted to members of the CDF collaboration as they may contain measurements that have not gone through CDF's rigorous internal review process. While an attempt has been made to only reference public notes, unfortunately it is necessary for this thesis to reference some of the private internal notes. To allow a proper academic evaluation of the work in this thesis, all CDF internal notes which are referenced by this thesis can be made available on request as long as the requester agrees to keep the contents confidential. 


\section{Chapter 2}

\section{Experimental Apparatus}

The data analysed in this analysis were collected by the Collider Detector at Fermilab (CDF) Collaboration, which runs a multipurpose detector, the CDF II Detector, observing proton-antiproton collisions with $\sqrt{s}=1.96 \mathrm{TeV}$ produced by the Tevatron collider. Currently the Tevatron is the highest energy hadron-hadron collider in the world, making it the best place to search for new physics beyond the Standard Model today. This chapter will briefly describe the key features of the Tevatron and the CDF detector, with more a detailed description of the CDF detector being available in [29].

\subsection{The Fermilab Accelerator Complex}

The Fermilab Accelerator complex consists of a series of accelerators which work in stages to produce $0.98 \mathrm{TeV}$ beams of protons and antiprotons. A schematic of the accelerator chain is shown in figure 2.1. The first accelerator is the Cockcroft-Walton pre-accelerator which creates negative hydrogen ions which are then accelerated to an energy of $750 \mathrm{keV}$. The hydrogen ions then pass into a $150 \mathrm{~m}$ long linear accelerator which raises the energy to $400 \mathrm{MeV}$. The ions are stripped of their electrons by passing them through a carbon foil before entering the Booster which accelerates resulting the protons to $8 \mathrm{GeV}$. The Booster then transfers the protons into the Main Injector, 7 bunches at a time with each bunch containing of order $10^{10}$ protons. The Main Injector is used to accelerate the protons to $150 \mathrm{GeV}$ while coalescing them into a single bunch in preparation for injection into the Tevatron. The process is repeated 36 times to obtain a total of 36 proton bunches in the Tevatron at an energy of $150 \mathrm{GeV}$.

The Main Injector is also responsible for sending $120 \mathrm{GeV}$ protons to the Antiproton Source. The Antiproton source produces antiprotons by colliding the protons into a nickel target and approximately 56,000 protons are required for every antiproton produced. Due to the production mechanism of antiprotons, the energy spread of the antiprotons in the beam is large. In order to reduce the energy spread, and thus increase the luminosity, the antiprotons are cooled using two separate cooling processes: 'stochastic cooling' [30] and 'electron cooling' [31]. In the stochastic cooling process, a detector measures the beam spread and then sends the information on how to re- 


\begin{tabular}{|c|c|c|}
\hline Parameter & Best Store & Fiscal Year 05 Average \\
\hline Protons per bunch & $263.1 \times 10^{9}$ & $232.4 \times 10^{9}$ \\
Antiprotons per bunch & $72.2 \times 10^{9}$ & $33.1 \times 10^{9}$ \\
Initial Luminosity & $225.2 \times 10^{30} \mathrm{~cm}^{-2} \mathrm{~s}^{-1}$ & $85.7 \times 10^{30} \mathrm{~cm}^{-2} \mathrm{~s}^{-1}$ \\
Integrated Luminosity per store & $8.1 \mathrm{pb}^{-1}$ & $2.9 \mathrm{pb}^{-1}$ \\
Store length & $32.0 \mathrm{hrs}$ & $24.5 \mathrm{hrs}$ \\
\hline
\end{tabular}

Table 2.1: The store parameters for the best ever store (29-30 July 2006) and the average for the 2005 fiscal year.

duce it to a magnet directly across the ring. The magnet then reduces the beam momentum spread by applying a correction based on that information to the particles when they arrive. The electron cooling process mixes the antiprotons with a beam of $4.3 \mathrm{MeV}$ electrons in a $20 \mathrm{~m}$ long cooling section. The antiprotons undergo Coulomb scattering in the electron beam, losing energy to the electrons in the processes. Eventually a thermal equilibrium is obtained, resulting in much smaller spread of antiproton energies.

The antiprotons are stored in the Accumulator ring until a sufficiently large 'stack' of antiprotons $\left(\mathcal{O} 10^{11}\right)$ has been accumulated. At this point the antiprotons are then injected into the Main Injector where they are organised into 4 bunches separated by $396 \mathrm{~ns}$. These bunches are accelerated to $150 \mathrm{GeV}$ and injected into the Tevatron in a similar manner to the protons. The process is repeated 9 times to give a total of 36 bunches in the Tevatron. The 36 antiproton bunches, together with the 36 proton bunches, are then accelerated from $150 \mathrm{GeV}$ to $980 \mathrm{GeV}$ at which point they are placed in a collision orbit to collide at the points where the CDF and D $\varnothing$ detectors are located. Once in a collision orbit, the beams are forced into collisions using the low beta quadrupole magnets which 'squeeze' the beam to reduce the transverse beam size.

The 36 bunches of protons and antiprotons form a 'store', which is maintained until the collisions and beam decay reduce the instantaneous luminosity enough to require a new store. A store lasts around a day and table2.1 gives the average values of the store parameters for the fiscal year 2005, as well as the parameters for the best store ever recorded as of September $1^{\text {st }}, 2006$. The typical number of antiprotons in the store has doubled in 2006, in part due to the introduction of electron cooling. This has helped to increase both peak and integrated luminosities. This increase can be seen in figure 2.2 which shows the peak and integrated luminosity delivered by the Tevatron over time. The efficiency of the CDF detector in recording the delivered luminosity varies, but is typically around $85 \%$. This inefficiency is in part due to dead-time inherent in the system and in part due to operational effects. 


\section{FERMILAB'S ACCELERATOR CHAIN}

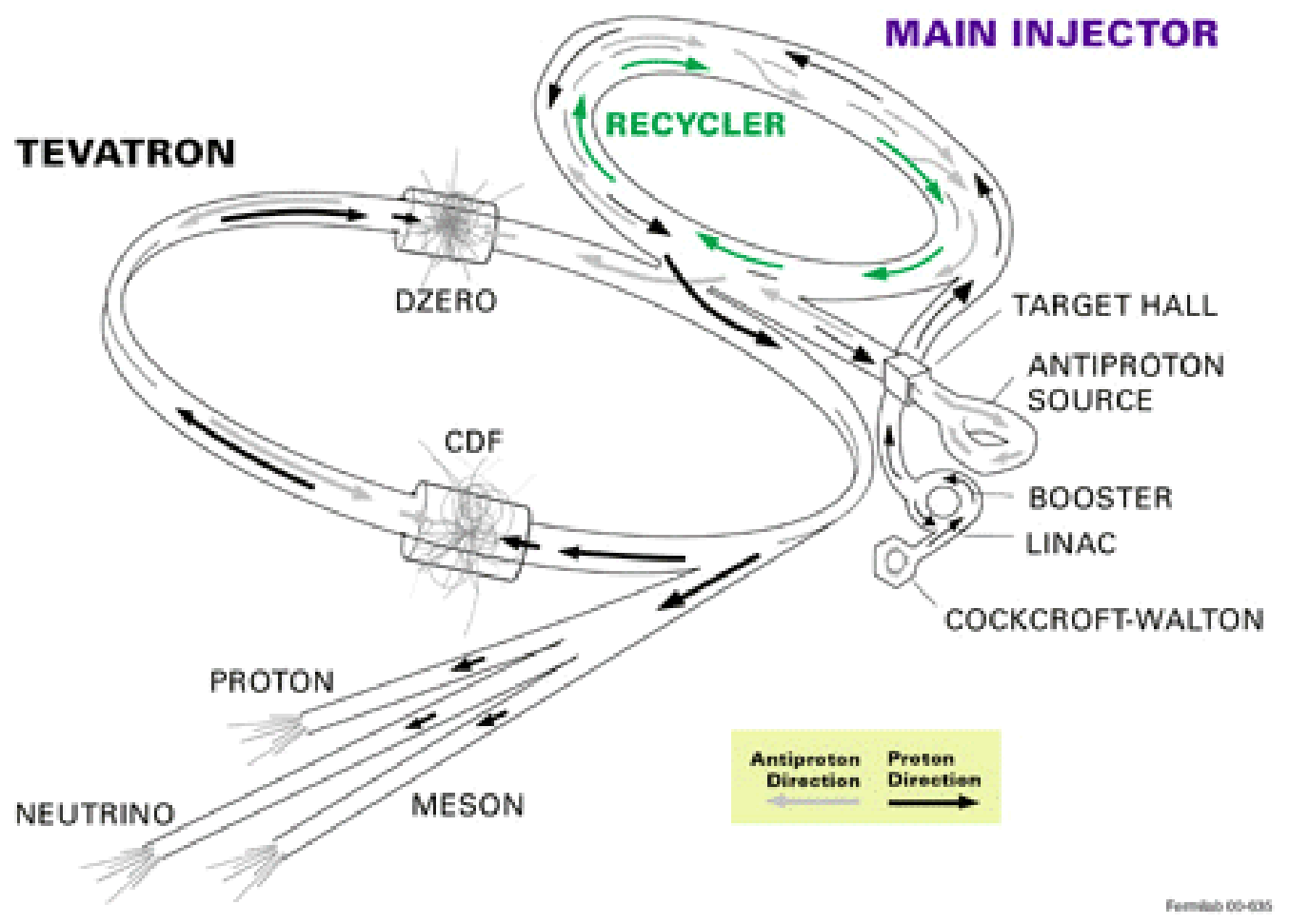

Figure 2.1: The Fermilab accelerator chain 


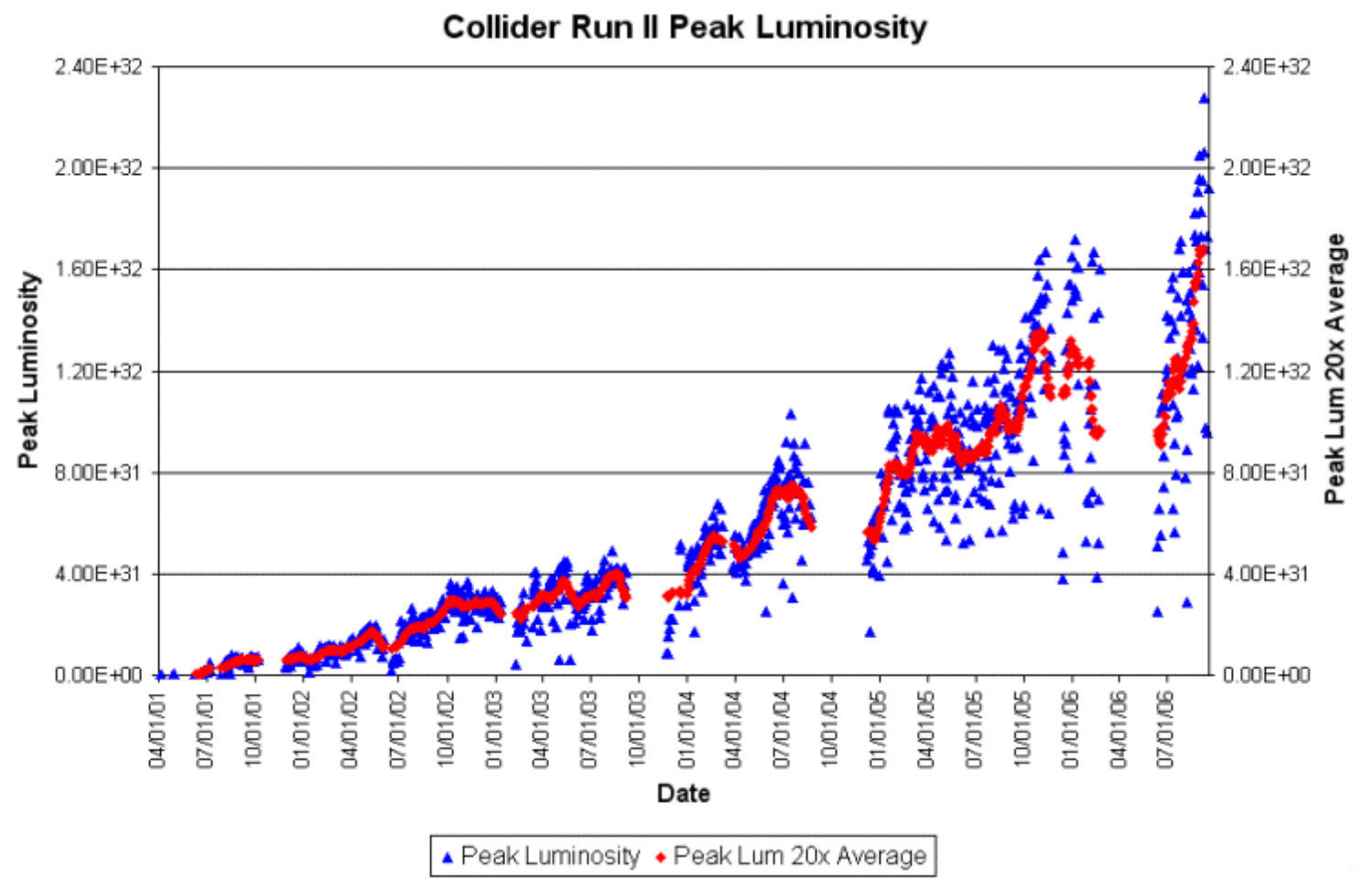

Collider Run II Integrated Luminosity

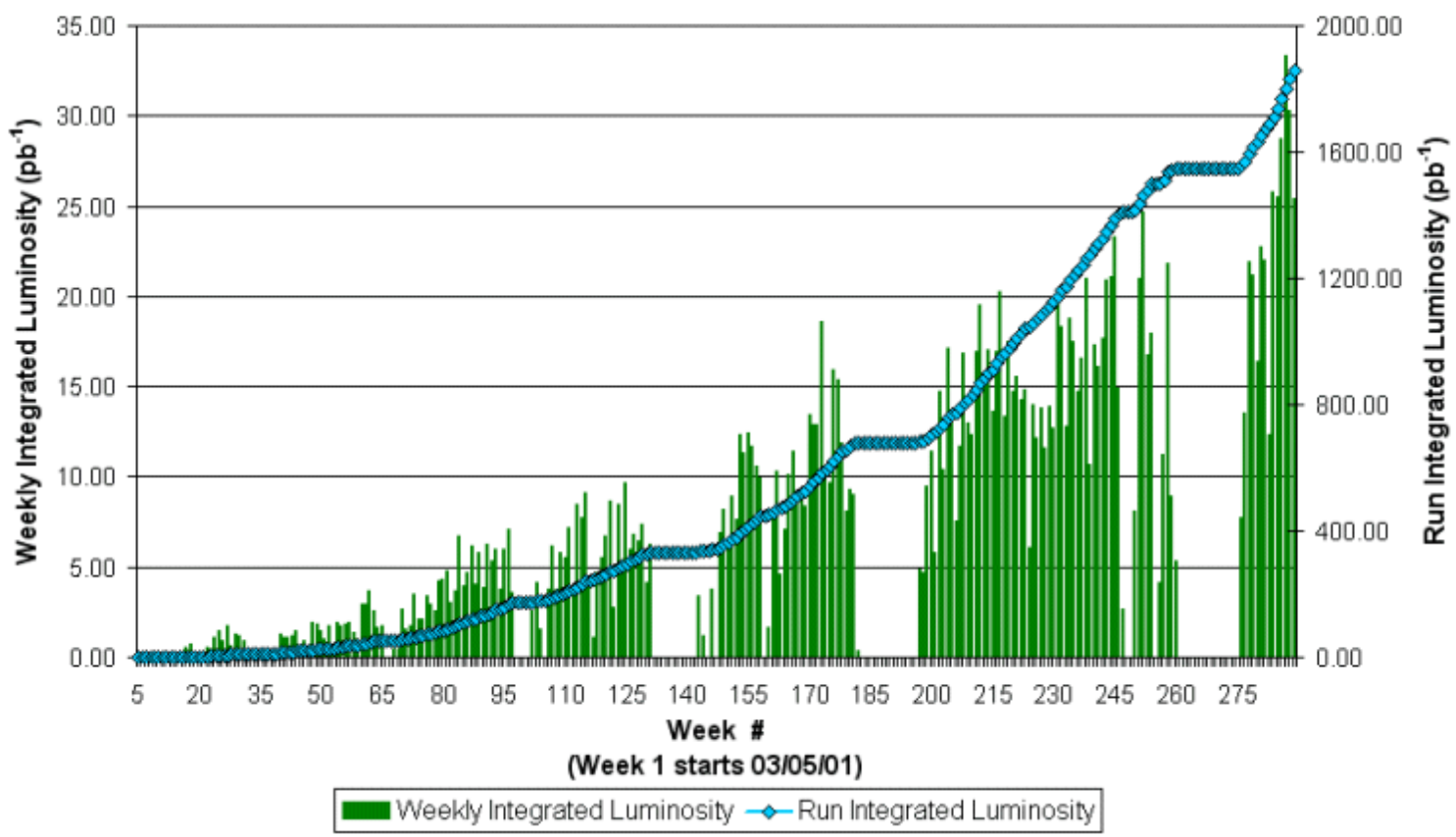

Figure 2.2: The integrated (top) and peak (bottom) luminosity delivered by the Tevatron over time 


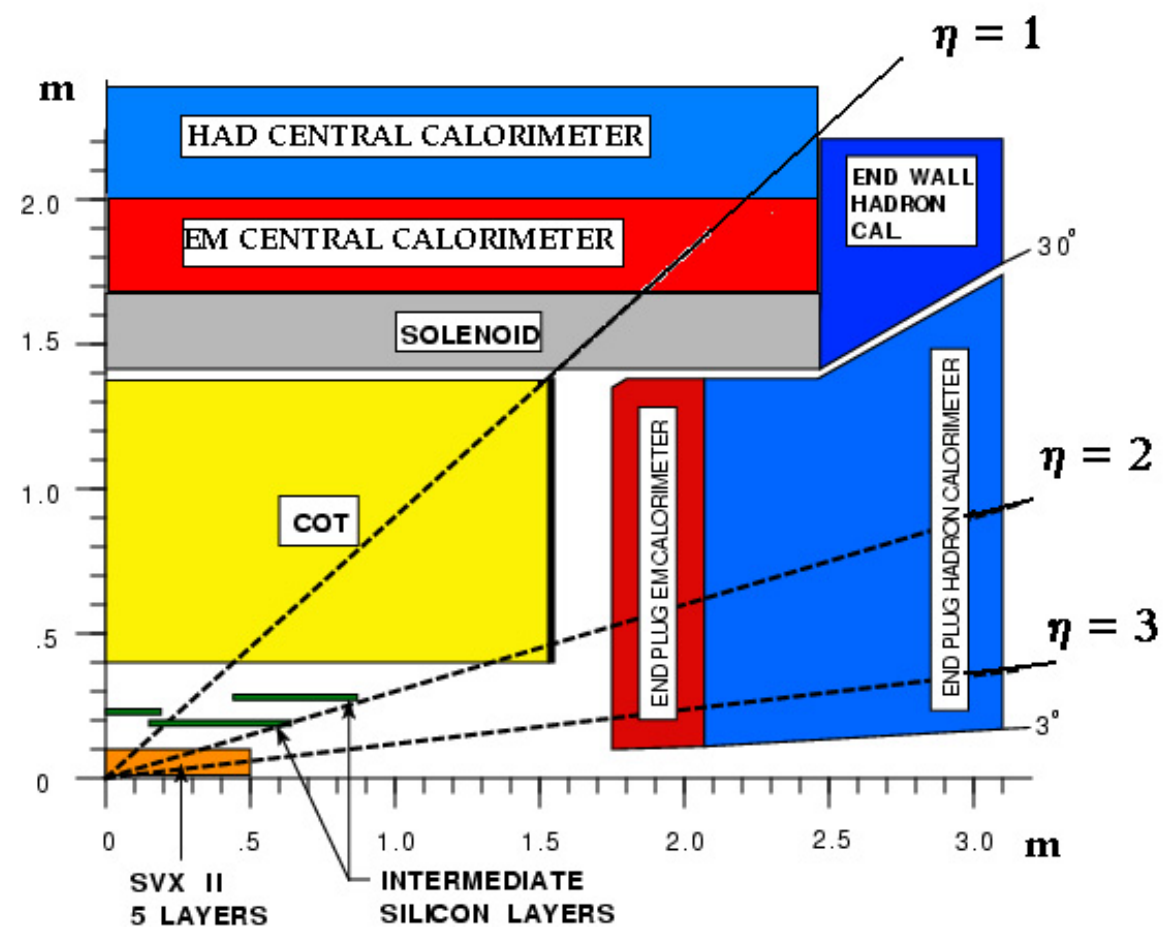

Figure 2.3: A side-on schematic view of the CDF II detector in the $y z$ plane as defined in section 2.2.1. Only one quadrant is shown, however the detector is rotationally symmetric about the $z$-axis and reflectionally symmetric about the $y$-axis. The muon chambers positioned around the hadronic calorimeters are not shown.

\subsection{The CDF II Detector}

The Collider Detector at Fermilab II detector is a general purpose physics detector observing the $1.96 \mathrm{TeV}$ centre of mass energy $p \bar{p}$ collisions produced by the Fermilab Tevatron. A side-on schematic view of the CDF detector is shown in figure 2.3. It principally consists of a tracking volume, a calorimeter system and a muon system.

\subsubsection{CDF Coordinate System}

The CDF coordinate system is a right handed coordinate system and is shown in figure 2.4. It is defined such that the direction of the protons, which travel clockwise around the ring, is along the positive z-axis. The positive $y$-axis is defined to be upwards which in turn defines the positive $x$-axis as pointing away from the centre of the ring. The azimuthal angle $(\phi)$ is defined to be the angle from the $x$-axis to the $y$-axis and the polar angle $(\theta)$ is defined to be the angle from the $z$-axis to the $y$-axis. This is used to define the transverse energy $\left(E_{T}\right)$ of a particle as $E_{T}=E \sin \theta$ where $E$ 


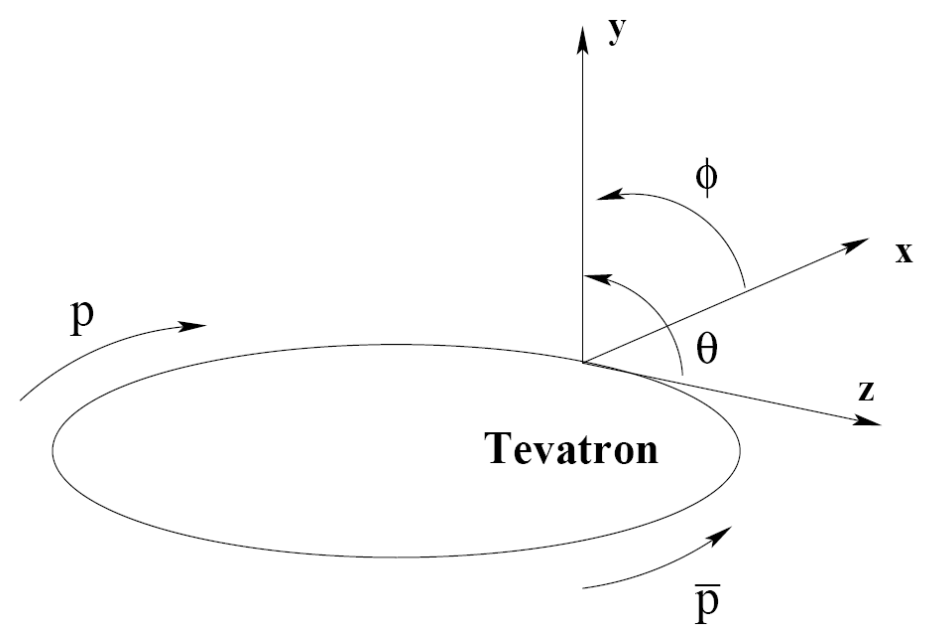

Figure 2.4: The CDF coordinate system

is the particle's energy. The polar angle is often expressed in terms of pseudorapidity $(\eta)$, which is defined as

$$
\eta=-\ln \tan \frac{\theta}{2}
$$

Pseudorapidity is the zero-mass limit of the quantity known as rapidity $(y)$ which is defined as

$$
y=\frac{1}{2} \ln \frac{E+p_{z}}{E-p_{z}}
$$

Pseudorapidity is either referred with respect to the event $\left(\eta_{\text {event }}\right)$ or with respect to the detector $\left(\eta_{\text {det }}\right)$. In the case of $\eta_{\text {event }}$, the pseudorapidity is calculated with respect to the $z$ vertex of the event, while for $\eta_{\text {det }}$, the pseudorapidity is calculated with respect to a $z$ vertex of zero, the nominal centre of CDF. Throughout this document, $\eta$ will be used as shorthand for $\eta_{\text {det }}$ as this is the more useful quantity as it relates directly to the part of the detector the electron is incident in, which in turn governs the energy corrections and selection criteria applied for that electron.

The separation of two objects in the detector is often expressed using the conical separation $(\Delta R)$ which is related to the difference in $\eta$ and $\phi$ as follows

$$
\Delta R=\sqrt{(\Delta \eta)^{2}+(\Delta \phi)^{2}}
$$

Finally, the terms east and west are sometimes used when referring to the two halves of the plug calorimeter. The positive $z$, and thus the positive $\eta$ side, is the east side with the negative $z$ side therefore being the west side. 


\subsubsection{Tracking System}

The CDF detector primarily uses a large open-cell drift chamber known as the Central Outer Tracker (COT) to obtain information about the trajectory of the charged particles within the detector. The COT is complemented by a system of silicon microstrip detectors, which is used to increase the accuracy of position measurements close to the interaction point. The silicon system is surrounded by the COT, which is in turn surrounded by a $5 \mathrm{~m}$ long superconducting solenoid which generates the 1.4 Tesla magnetic field, which is used to obtain a momentum measurement.

Charged particles passing through the tracking volume can be detected either through the ionisation of material, whether it be the silicon in the silicon tracker or the atoms in the gas filling the drift chamber. The subsequent detection of the particle as it passes though the tracking volume allows the particle's trajectory to be recorded. The magnetic field present in the tracking volume causes the charged particles' trajectories to curve and travel in a helical path. The curvature each particle experiences is inversely proportional to the particle's transverse momentum $\left(p_{T}\right)$, allowing the momentum to be measured using the relationship below

$$
p_{T}=0.3 B R_{c}(\mathrm{GeV} / c)
$$

where $B$ is the magnetic field strength in Tesla and $R_{c}$ is the radius of curvature in metres. This relationship results in an increasing inaccuracy of the momentum measurement as track $p_{T}$ increases since the radius of curvature becomes increasingly larger and therefore more difficult to measure.

\section{Silicon Tracking System}

The silicon tracking system consists of three subsystems. Figure 2.5 shows an endon schematic of the complete system. The innermost subsystem, known as Layer 00, consists of a layer of radiation hard silicon wafers mechanically attached to the beam pipe at a radius of $1.35 \mathrm{~cm}$. The layout of the silicon wafers can be seen more clearly in figure 2.6.

The second subsystem is the Silicon Vertex Detector (SVX II). This consists of three identical cylindrical barrels with a total length of $96 \mathrm{~cm}$. Each barrel consists of 5 layers of double-sided silicon microstrip detectors arranged into 12 ladders in $\phi$ whose layout can be seen in figure 2.6. The double-sided design of the ladders enables each ladder to have an axial and stereo side. The $x-y$ position of the particle is measured by the axial sides which are angled parallel to the $z$-axis. The $z$ position information is obtained from the stereo sides of layers 0,1 and 3 which are angled perpendicular to the $z$-axis. The stereo sides of layers 2 and 4 are angled at $-1.2^{\circ}$ and $+1.2^{\circ}$ respectively to allow the tracking algorithm to match better the stereo hits of layers 0,1 and 3 to the axial hits. The estimated intrinsic impact parameter resolution for the SVX II is $9 \otimes 50 / p_{T} \mu \mathrm{m}$ without Layer 00 and $6 \otimes 25 / p_{T} \mu \mathrm{m}$ with Layer 00 [32].

The outermost subsystem, known as the Intermediate Silicon Layers (ISL) is designed to supplement the COT up to $|\eta| \approx 1$ and act as a replacement past that point. 


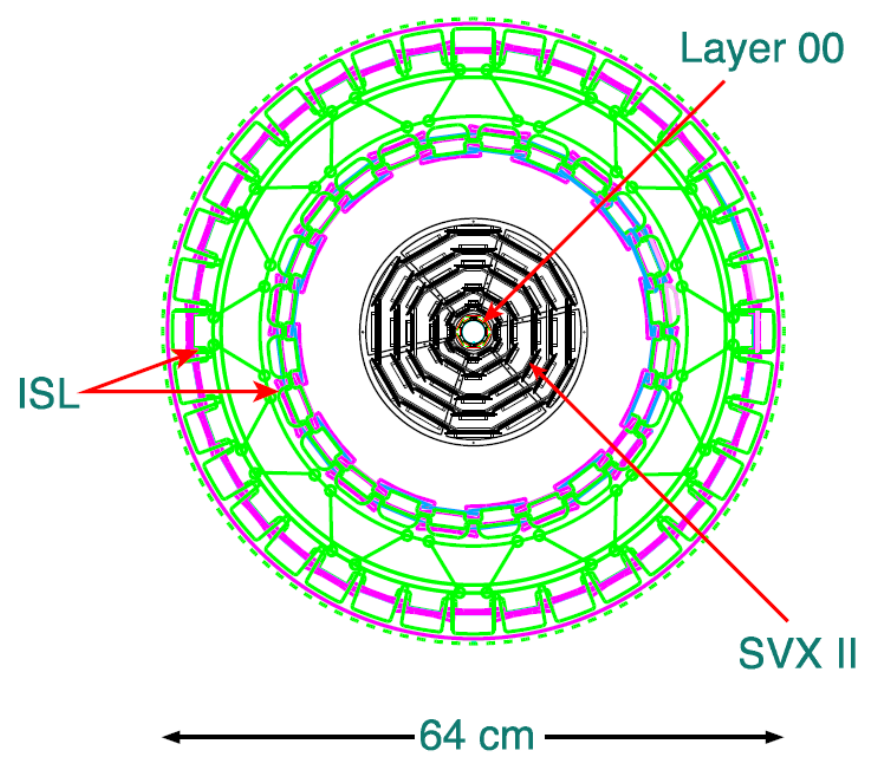

Figure 2.5: An end-on view of the complete CDF silicon system consisting of Layer 00, SVX II and ISL.

The ISL has one central layer at a radius of $22 \mathrm{~cm}$ covering the region $|\eta| \leq 1.0$ and two forward layers at radii of $20 \mathrm{~cm}$ and $28 \mathrm{~cm}$ respectively, with both covering the region $1.0 \leq|\eta| \leq 2.0$. This enables the tracking coverage to be extended from $|\eta| \approx 1$ to $|\eta| \approx 2$. The extension of the $\eta$ coverage can best be seen in figure 2.3 .

\section{Central Outer Tracker}

The Central Outer Tracker is a 3m long 96 layer open-cell drift chamber whose active region is $40 \leq y \leq 132 \mathrm{~cm}$, giving a tracking coverage of $\eta \leq 1.0$ if a hit in each layer is required. It is organised into 8 'superlayers': 4 axial and 4 stereo. Each superlayer consists of 25 wires: 13 potential wires and 12 sense wires. The stereo layers are alternately angled by $\pm 3^{\circ}$ from the axial layers to provide $z$ information. The layout of the superlayers can be seen in figure 2.7. The tracking volume is filled with a mixture of $60 \%$ argon and $40 \%$ ethane with a small amount of alcohol. The track momentum resolution of the COT is $\frac{\sigma\left(p_{T}\right)}{p_{T}} \sim 0.15 \% \cdot p_{T}(\mathrm{GeV} / c)$.

During the period of Feb 13 to May 26 2004, the rate of reduction in the gain of the inner superlayers was faster than had been expected from the normal ageing processes affecting the wires. This was investigated and was found to be the result of small deposits of material from the hydrocarbons in the gas growing on the wires [33]. The process was completely reversed by increasing the gas flow and adding a small (70-80 ppm) amount of oxygen to the gas mixture. However this does mean that the data taken during this period have reduced tracking resolution and are therefore not used in this analysis. 


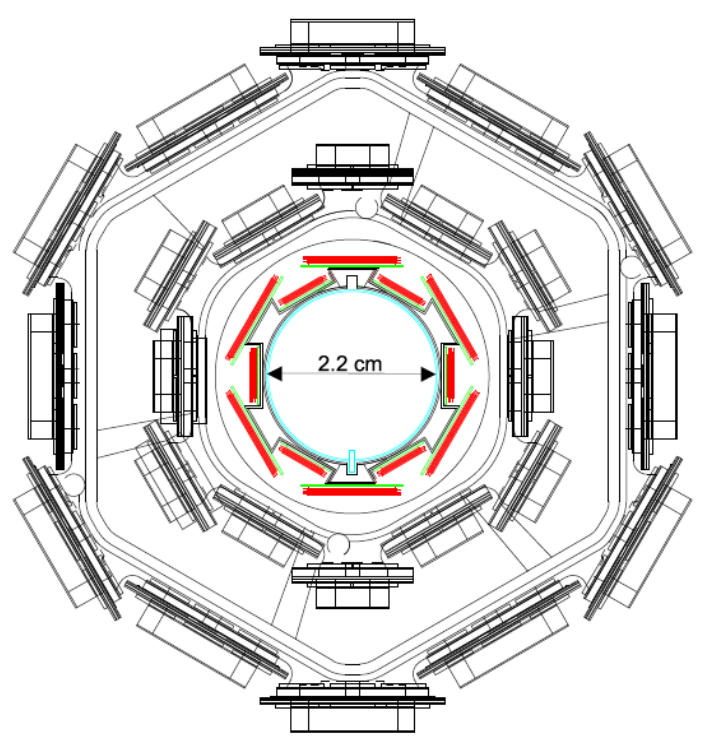

Figure 2.6: An end-on view of Layer 00, shown in red and green, and the first two layers of the SVX II detector

\subsubsection{Calorimeter System}

The CDF calorimeter is a sampling calorimeter in which the energy deposits of a particle are sampled at regular intervals by the use of scintillators and photomultiplier tubes to give a measurement of the energy of the incident particle. The rate and mechanism of the particle's energy deposition in the calorimeter depends on the particle and these mechanisms are summarised in the next section.

There are two separate calorimeter systems, the central calorimeter which covers $|\eta| \leq 1.1$ and the plug calorimeter which covers $1.1 \leq|\eta| \leq 3.6$. The plug calorimeter is composed of two separate identical calorimeters, one for the west side and one for the east side. Each calorimeter system has both electromagnetic and hadronic sections. The electromagnetic sections are equipped with proportional strip and wire chambers to measure the position of an incident electromagnetic shower and a pre-radiator to help separate electrons and photons from neutral pions. The main characteristics of the calorimeter systems are summarised in table 2.2, $X_{0}$ and $\Lambda_{0}$ refer to the radiation and nuclear interaction lengths and characterise the scales of the electromagnetic and hadronic showers respectively. They are explained in more detail in the following section.

\section{Interaction of Particles with Matter}

Electrons interact with matter via ionisation, Møller scattering, Bhabha scattering, pair annihilation and bremsstrahlung. Photons interact with matter via the photoelectric effect, Compton scattering, Rayleigh scattering, photon nuclear absorption and 


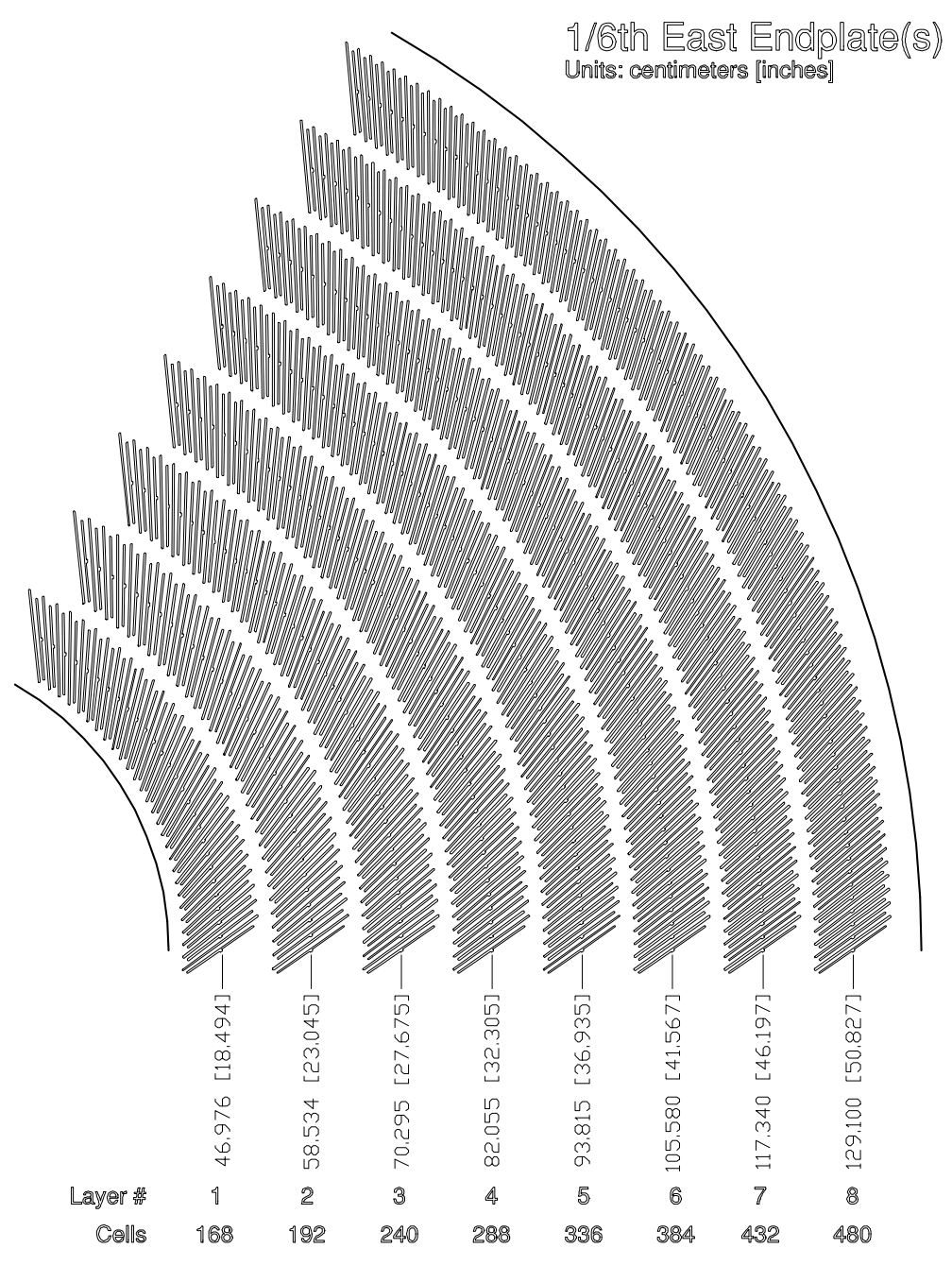

Figure 2.7: One eighth of the east COT end-plate showing the 8 superlayers and cell layout. The stereo layers are odd numbered while the axial layers are even numbered.

\begin{tabular}{|c|c|c|c|c|c|}
\hline Sub Detector & CEM & CHA & WHA & PEM & PHA \\
\hline Coverage & $|\eta| \leq 1.1$ & $|\eta| \leq 0.9$ & $0.7 \leq|\eta| \leq 1.3$ & $1.1 \leq|\eta| \leq 3.6$ & $1.1 \leq|\eta| \leq 3.6$ \\
Layers & 31 & 32 & 15 & 23 & 23 \\
Material & Lead & Steel & Steel & Lead & Iron \\
Depth & $18 X_{0}$ & $4.7 \Lambda_{0}$ & $4.5 \Lambda_{0}$ & $21 X_{0}$ & $7 \Lambda_{0}$ \\
$\frac{\sigma(E)}{E}$ & $\frac{13.5 \%}{\sqrt{E \sin \theta}} \oplus 2 \%$ & $\frac{80 \%}{\sqrt{E}}$ & $\frac{80 \%}{\sqrt{E}}$ & $\frac{14.4 \%}{\sqrt{E}} \oplus 0.7 \%$ & $\frac{80 \%}{\sqrt{E}} \oplus 5 \%$ \\
\hline
\end{tabular}

Table 2.2: Summary of the CDF calorimeters. For the energy resolutions, $E$ is in units of $\mathrm{GeV}$. The individual sub detectors CEM, CHA, etc are described in the text. 
pair production. At low energies, electrons primarily lose energy via ionisation, while photons primarily lose energy via the photoelectric effect. At high energies, the dominate energy loss mechanism is bremsstrahlung for electrons and pair production for photons. The electron energy at which the rate of energy loss for an electron via bremsstrahlung is equal to the rate of energy loss via ionisation is known as the critical energy $\left(E_{c}\right)$. The critical energy is approximately $E_{c}=(800 \mathrm{MeV}) /(Z+1.2)$ [4], where $Z$ is the atomic number of the material the electron is interacting with. The passage of high energy photons and electrons with $E>E_{c}$ through a material will lead to an electromagnetic shower. Both bremsstrahlung and pair production produce additional photons and electrons which in turn produce further photons and electron pairs. Each of these subsequent electrons and photons will be produced with a lower energy than the parent particle until eventually the electrons and photons are produced with $E<E_{c}$. At this point, the ionisation and the photoelectric effect energy loss mechanisms become dominant and the shower begins to decay. The length of the shower is dictated by the radiation length $\left(X_{0}\right)$ of the material. The radiation length is defined as the mean distance over which a high energy electron loses all but $1 / e$ of its energy. It is also approximately equal to $7 / 9$ of the mean free path for pair production by a high energy photon. The radiation length of a material depends on its atomic number, with a high atomic number material having a smaller radiation length. The radiation length for lead, a commonly used material for electromagnetic calorimeters, is approximately $0.56 \mathrm{~cm}$ with a $E_{c} \approx 7.6 \mathrm{MeV}$.

Hadronic particles $(\pi, p, \bar{p}, K)$ interact strongly with the nuclei of the atoms of the material they are passing though. Charged hadronic particles can also interact via ionisation, bremsstrahlung and other electromagnetic processes but none of these processes account for a significant amount of energy loss for hadrons until an energy of about several hundred $\mathrm{GeV}$, at which point energy loses from bremsstrahlung start to become important. Similarly to electrons and photons, the passage of a hadron through a material creates a shower of secondary particles. Hadronic interactions have a lower probability per unit length of the absorber of occurring than the radiative and pair production interactions of electrons and photons and therefore a hadronic shower typically happens over a larger scale than an electromagnetic shower. The scale of a hadronic shower in a material is set by its nuclear interaction length $\left(\Lambda_{0}\right)$. The nuclear interaction length of a material depends on its atomic mass, with a high atomic mass material having a smaller nuclear interaction length. The nuclear interaction length in iron, a commonly used material for hadronic calorimeters, is approximately $16.6 \mathrm{~cm}$. This approximately 30 times longer than the radiation length and therefore electromagnetic particles will be mostly contained in the front section of the calorimeter while hadronic particles will deposit the majority of their energy over the rest of the calorimeter.

Muons, unlike electrons, are too heavy for bremsstrahlung to be a significant energy loss mechanism at current collider energies and as a consequence they lose energy primarily by ionisation. The rate of energy loss is therefore small and so coupled with their relatively long life time, a muon will usually escape the detector, leaving only a 
minimum ionising track in any tracking volume it passes through.

\section{Central Electromagnetic Calorimeter}

The Central Electromagnetic Calorimeter (CEM) is a sampling calorimeter consisting of 31 layers of $5 \mathrm{~mm}$ thick scintillator interleaved with $303 \mathrm{~mm}$ thick layers of lead giving a total thickness of 18 radiation lengths. The CEM is segmented into ten equal towers in $\eta$, with each tower subdivided into $15^{\circ}$ segments in $\phi$. The tower organisation can also be referred to in terms of $\phi$ wedges, with each $\phi$ wedge composed of all the tower segments at the same value of $\phi$. The energy resolution is measured to be $\frac{\sigma(E)}{E} \sim \frac{13.5 \%}{\sqrt{E \sin \theta}} \oplus 2 \%$, with $E$ in $\mathrm{GeV}[34]$.

At approximately 6 radiation lengths into the calorimeter, which is the point of the shower maximum, there is a proportional strip and wire chamber known as the Central Electromagnetic Shower Maximum Detector (CES). The CES consists of orthogonal strips and wires in an $\mathrm{Ar} / \mathrm{CO}_{2}$ gas mixture with the wires running parallel to the beam axis. The CES is used to obtain a position measurement of the electromagnetic shower near the shower maximum with an accuracy of about $2 \mathrm{~mm}$ for $50 \mathrm{GeV}$ electrons [34]. The CES is also used to measure the profile of the shower shape and this can be compared to measurements from test beam data to provide an additional discriminator to separate electrons and photons from hadronic particles.

\section{Plug Electromagnetic Calorimeter}

The Plug Electromagnetic Calorimeter (PEM) is a sampling calorimeter consisting of 23 layers, each composed of a $4.5 \mathrm{~mm}$ thick layer of lead and a $4 \mathrm{~mm}$ thick layer of scintillator, to give a total thickness of about 21 radiation lengths. The PEM is segmented into towers, with the lower towers in $\eta$ being $15^{\circ}$ in $\phi$ and the upper towers in $\eta$ being $7.5^{\circ}$ in $\phi$. The precise segmentation of the PEM is shown in figure 2.8.

The energy resolution of the PEM is measured to be $\frac{\sigma(E)}{E} \sim \frac{14.4 \%}{\sqrt{E}} \oplus 0.7 \%$, with $E$ in $\mathrm{GeV}[35]$.

The first layer of the PEM is read out separately as the Plug Pre-Radiator (PPR) to help separate electrons and photons from pions. At approximately six radiation lengths deep there is a shower maximum detector, known as the Plug Electromagnetic Shower Maximum Detector (PES). The PES consists of two layers of $5 \mathrm{~mm}$ wide scintillator strips at $45^{\circ}$ to each other and can measure the shower's transverse position to an accuracy of typically $1 \mathrm{~mm}$.

\section{Central Hadronic Calorimeter}

The Central Hadronic Calorimeter (CHA) consists of 32 layers of $2.5 \mathrm{~cm}$ steel interleaved with $1.0 \mathrm{~cm}$ thick layers of scintillator, corresponding to a thickness of about 4.7 nuclear interaction lengths. The segmentation of the CHA is identical to the CEM, 10 towers in $\eta$ with each tower divided into $15^{\circ}$ segments in $\phi$. As can be seen from 


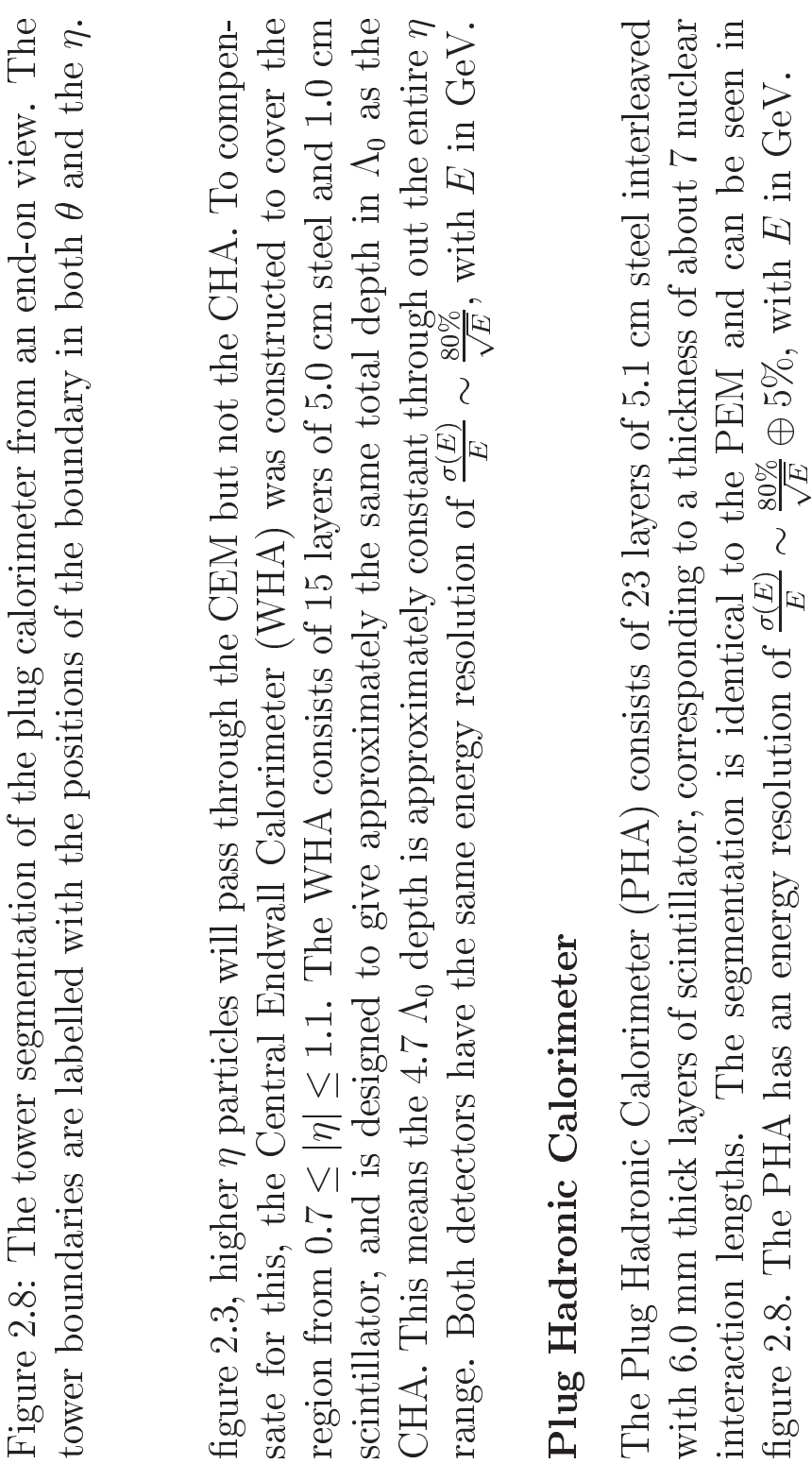

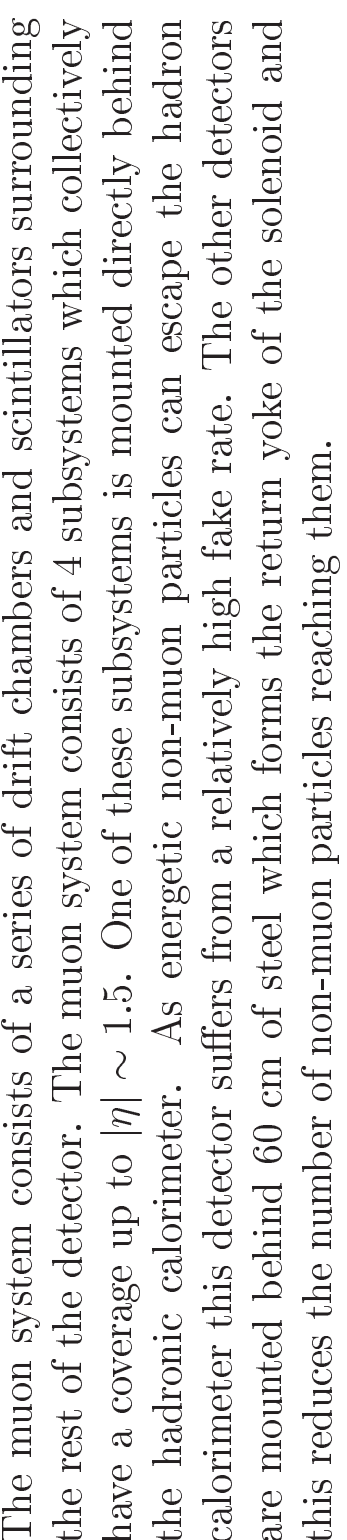


Hits in the detectors are combined into a 'stub' which is then matched with a track in the COT to form a muon candidate. The muon systems are not utilised in this analysis for any purpose and are not required to be functioning during data taking.

\subsubsection{Luminosity Monitoring}

The instantaneous luminosity recorded by the experiment is measured using inelastic $p \bar{p}$ interactions. The rate of inelastic $p \bar{p}$ interactions is measured using the Cherenkov Luminosity Counter (CLC) [36]. The CLC consists of two modules placed between the beam-pipe and the plug calorimeter in the region of $3.7 \leq|\eta| \leq 4.7$ on the separate sides of the detector. Each module consists of 48 conical gas-filled Cherenkov counters, arranged in three concentric circles.

The luminosity can be calculated from the number of hits in the CLC by the following equation

$$
L=\frac{f N_{H}}{\sigma_{p \bar{p}} A_{C L C} N_{H}^{1}}
$$

where $f$ is the frequency of the bunch crossings, $\sigma_{p \bar{p}}$ is the inelastic $p \bar{p}$ cross-section, $A_{C L C}$ is the CLC acceptance, $N_{H}$ is the number of hits in the CLC for a bunch crossing and $N_{H}^{1}$ is the number of hits in the CLC for a single $p \bar{p}$ collision. The inelastic $p \bar{p}$ crosssection is extracted from the measured rates of elastic and inelastic $p \bar{p}$ collisions using the procedure in [37]. The measured uncertainty on the inelastic $p \bar{p}$ cross-section is $4 \%$ and the uncertainty on the CLC acceptance is $4.4 \%$ [37]. This gives a total uncertainty on the luminosity of $6 \%$.

\subsubsection{Trigger System}

Collisions occur at CDF approximately at a rate of $2.5 \mathrm{MHz}$. The majority of these collisions result in minimum bias 1 events since events such as $Z^{0} / \gamma^{*} \rightarrow e^{+} e^{-}$, $t \bar{t}$, or b physics events have cross-sections many orders of magnitude lower than the total inelastic $p \bar{p}$ cross-section of $60.7 \pm 2.4 \mathrm{mb}$ [37]. Ideally every event would be stored for further analysis, however the bandwidth and storage this would require makes this unfeasible. The goal of the trigger system is to reduce the bandwidth and required storage to manageable levels while still selecting as many of the interesting events as possible. The trigger system does this by looking at the event and seeing if it matches to one of its pre-programmed signatures of interesting events such as 2 high- $p_{T}$ leptons in the event. However, with a new event every $0.4 \mu \mathrm{s}$, the trigger system only has a finite amount of time to make a decision. This time can be extended by buffering events, but this is not sufficient to allow the second or so it would take to fully reconstruct the event and determine whether it is interesting or not. Therefore, a three level trigger system is utilised and a schematic of this trigger system is shown in figure 2.9. Each level

\footnotetext{
${ }^{1} \mathrm{~A}$ minimum bias event is any soft $\mathrm{Q} C D$ event. Typically there are $\sim 2$ minimum bias events per bunch crossing.
} 
reduces the rate so that the next level has more time to reconstruct the event in order to make a more sophisticated decision. For example, an electron signature at Level 1 is simply a single calorimeter tower and a low resolution 2D track, while at Level 2 there is time to perform a simple clustering algorithm and use information from the shower maximum detectors. The first level reduces the total rate from approximately $2.5 \mathrm{MHz}$ to $\sim 30 \mathrm{kHz}$. The second level reduces this rate to $\sim 350 \mathrm{~Hz}$, which is finally reduced after Level 3 to a rate of $\sim 75 \mathrm{~Hz}$. The maximum tape writing rate is $10 \mathrm{~Hz}$ and so to achieve this the Level 3 output is split into 8 separate streams.

\section{Level 1 Trigger}

The Level 1 trigger consists of custom built hardware to obtain the fastest possible performance. Each detector component has a 42 cell pipeline synchronised to the Tevatron master clock which has a period of 132 ns. A decision on whether to accept or reject the event must be made before the data reach the end of the pipeline, otherwise the data are lost. Therefore the Level 1 trigger only has $\sim 5.5 \mu$ s in which to make a decision. To achieve this it utilises 3 parallel processing paths, with each path being dedicated to reconstructing either calorimeter objects, muons or tracks. Tracking information is available at Level 1 from the eXtremely Fast Tracker (XFT) which compares the COT hits to predefined lookup tables in order to define 2D tracks. Calorimeter objects consist of a single tower and muons consist of a muon stub matched to an XFT track within $2.5^{\circ}$ in $\phi$. The Level 1 trigger reduces the initial $2.5 \mathrm{MHz}$ rate to $\sim 30 \mathrm{kHz}$.

\section{Level 2 Trigger}

The Level 2 trigger, like the Level 1 trigger, also consists of custom built hardware. Events passing the Level 1 trigger are written to one of the four data buffers of the Level 2 trigger. Unlike the Level 1 pipeline, data will remain in the Level 2 data buffers until a trigger decision to accept or reject the event is made. This means that if a Level 1 accept occurs when all four buffers are in use, dead-time will occur. The Level 2 trigger is required to make a decision within approximately $20 \mu$ s to minimise this deadtime. This is sufficient time to do jet clustering and basic calorimeter clustering with shower maximum detector information added. Tracking resolution is also improved over Level 1 and information from the SVX II is available. Finally the matching of the muon stubs to tracks is improved. The Level 2 trigger reduces the rate to $\sim 250 \mathrm{~Hz}$.

\section{Level 3 Trigger}

The Level 3 trigger consists of the event builder and the Level 3 processing farm. The event builder consists of custom hardware which constructs the complete event from all the separate detector systems while the Level 3 processing farm consists of commodity PCs. The Level 3 processing farm is separated into 16 different sub-farms, with each sub-farm consisting of 16 dual CPU nodes. The Level 3 trigger has approximately a second to make a decision, which is sufficient time to almost fully reconstruct the event 


\section{Dataflow of CDF "Deadtimeless" Trigger and $D A Q$}

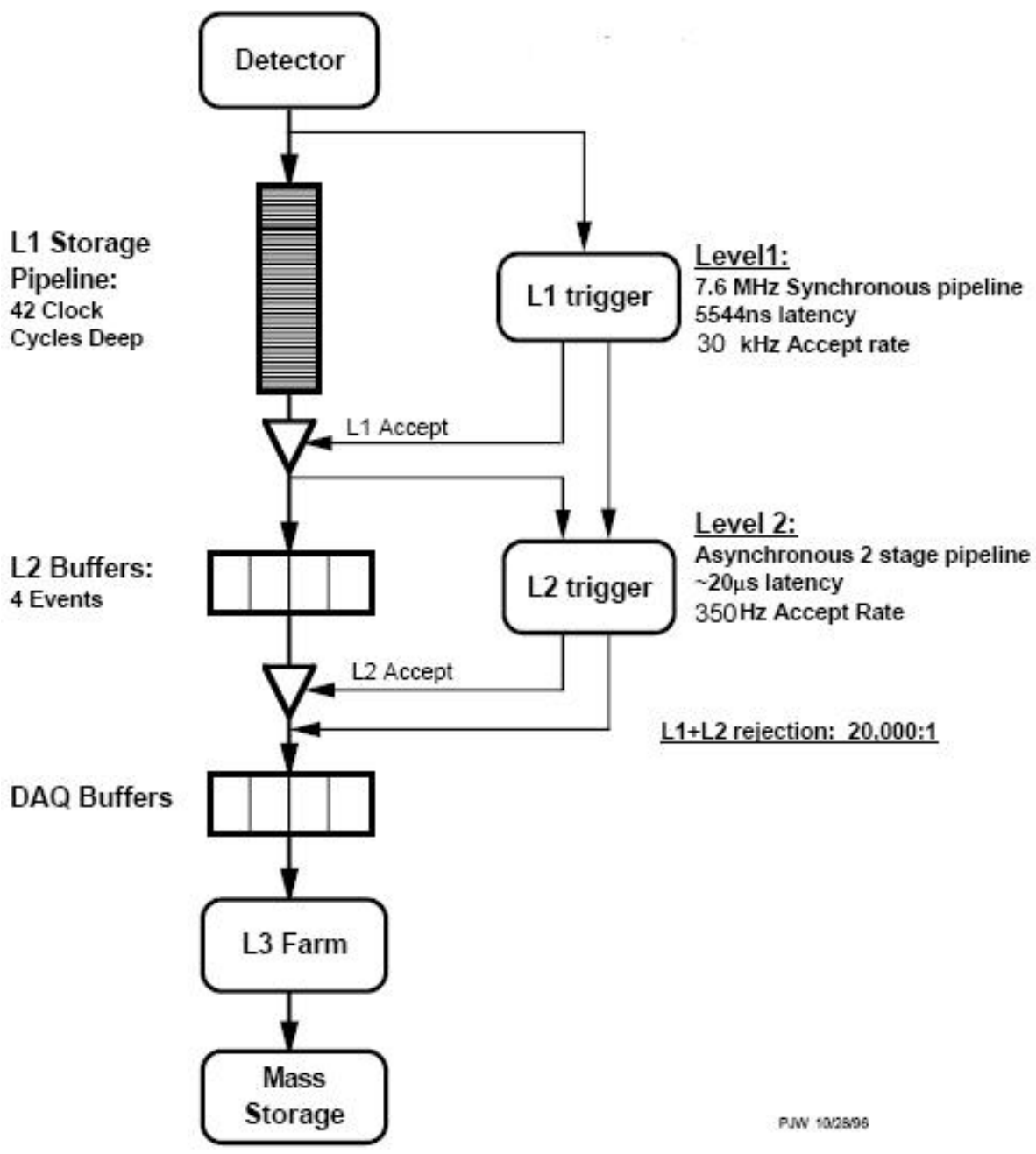

Figure 2.9: A flow diagram of the CDF trigger system. 
using reconstruction algorithms very similar to the offline processing algorithms. The event is not $100 \%$ fully reconstructed as there are calibrations that require a second pass over the data, such as the energy scale factors in table 4.1, which cannot be applied until a later time. By almost fully reconstructing the event, the trigger decision can be made with detailed particle identification cuts and event topologies. The Level 3 trigger reduces the event rate to $\sim 75 \mathrm{~Hz}$.

\subsubsection{Offline Processing and Software}

The CDF software is a custom designed, object orientated framework written in the $\mathrm{C}++$ programming language. It relies heavily upon the ROOT data analysis package [28] for data handling. The software takes the output of the detector and performs object reconstruction, such as electron clustering and track finding. It also applies the majority of the energy and momentum calibrations required.

The CDF software is continually evolving. However electron reconstruction has been relatively stable throughout this analysis and is expected to remain so until the next and final major update when the detector simulation will be tuned to take account of data-Monte Carlo event discrepancies found during the course of this analysis. The versions of the CDF software used in this analysis are versions 5.3.1 to 6.1.4, during which no major changes were made to electron reconstruction or simulation. In version 6.1 of the CDF software, improved tracking algorithms were introduced which consequently improve the CES-track matching variables discussed later, but this has an a negligible effect in the context of this analysis. The other significant change was that the CDF software framework version 6.1 is compiled under the GCC compiler, rather than the KCC compiler which was used for all previous versions. Minor version numbers include simple bug fixes, often for muon or jet reconstruction and have little effect on this analysis. The version number used for various parts of the analysis is included for completeness only and imparts little practical information to the non-CDF reader. A full listing of all CDF software code can be found at [38]. 


\section{Chapter 3}

\section{Triggers and Datasets}

This analysis uses $819 \mathrm{pb}^{-1}$ of $p \bar{p}$ collision data, with the data sample used being discussed in section 3.1. The various triggers used to select the data are discussed in section 3.2. Finally the Monte Carlo simulated samples used to estimate such things as the signal selection efficiency, the predicted signal distributions and the backgrounds to di-electron pairs, are discussed in 3.3 .

\subsection{Data Sample}

The data used in this analysis were collected over a time period from March 2002 to August 2005 by the CDF detector, which corresponds to a total integrated luminosity of $819 \mathrm{pb}^{-1}$ after data quality requirements. To ensure data quality, the calorimeter and the COT are required to have been functional at the time the data were taken. This corresponds to the run being marked as 'good' for electrons by the CDF good run group [40]. Some studies done as part of this analysis require 'Phoenix Tracking' which is explained in section 4.2.3. For these studies, the silicon system is required to have been fully functional at the time the data were taken, corresponding to the run being marked as good for silicon. This requirement reduces the total integrated luminosity available to $751 \mathrm{pb}^{-1}$.

\subsection{Triggers}

Three triggers are used in this analysis; the ELECTRON_CENTRAL_18, ELECTRON70_L2_JET and Z_NOTRACK triggers. The primary trigger is the Z_NOTRACK trigger which requires two EM calorimeter clusters with $E_{T} \geq 18 \mathrm{GeV}$. The ELECTRON_CENTRAL_18 and ELECTRON70_L2_JET triggers are used as backup triggers and both require just a single EM calorimeter cluster but with an associated track. The ELECTRON70_L2_JET requires a higher $E_{T}$ EM calorimeter cluster but otherwise has looser selection requirements than the ELECTRON_CENTRAL_18 trigger. Full details of the trigger requirements can be found in table 3.1. The Z_NOTRACK trigger is $\sim 100 \%$ efficient in the electron energy 


\begin{tabular}{|c|c|c|c|}
\hline & Z_NOTRACK & ELECTRON_CENTRAL_18 & ELECTRON70_L2_JET \\
\hline L1 & $\begin{array}{c}1 \text { EM Cluster } \\
E_{T}>8 \mathrm{GeV} \\
\mathrm{had} / \mathrm{em}<0.125\end{array}$ & $\begin{array}{c}1 \text { CEM Cluster } \\
E_{T}>8 \mathrm{GeV} \\
\text { XFT } p_{T}>8.36 \mathrm{GeV} / \mathrm{c} \\
\text { had } / \text { em }<0.125\end{array}$ & $\begin{array}{c}1 \text { Jet } \\
E_{T}>10 \mathrm{GeV}\end{array}$ \\
\hline L2 & $\begin{array}{c}2 \text { EM Clusters } \\
E_{T}>16 \mathrm{GeV} \\
\mathrm{had} / \mathrm{em}<0.125\end{array}$ & $\begin{array}{c}1 \text { CEM Cluster } \\
E_{T}>16 \mathrm{GeV} \\
\mathrm{XFT} p_{T}>8 \mathrm{GeV} / \mathrm{c} \\
\mathrm{had} / \mathrm{em}<0.125\end{array}$ & $\begin{aligned} & 1 \text { Jet } \\
& E_{T}> 90 \mathrm{GeV} \\
&- \\
&-\end{aligned}$ \\
\hline L3 & $\begin{array}{c}2 \text { EM Clusters } \\
E_{T}>18 \mathrm{GeV}\end{array}$ & $\begin{array}{c}1 \text { CEM Cluster } \\
E_{T}>18 \\
\text { COT Track } p_{T}>9 \mathrm{GeV} / \mathrm{c} \\
\text { had } / \mathrm{em}<0.125 \\
L_{S h r}<0.4 \\
\mathrm{CES} \Delta Z<8\end{array}$ & $\begin{array}{c}\text { 1 CEM Cluster } \\
E_{T}>70 \mathrm{GeV} \\
\text { COT Track } p_{T}>15 \mathrm{GeV} / \mathrm{c} \\
\text { had } / \mathrm{em}<0.2\end{array}$ \\
\hline
\end{tabular}

Table 3.1: Analysis trigger requirements. Note that the $L_{S h r}$ and CES $\Delta Z$ ELECTRON_CENTRAL_18 requirements only apply to data taken after April 2003. The quantities had/em, $L_{S h r}$ and CES $\Delta Z$ are defined in section 4.1.4.

range considered in this analysis for events with two central electrons [39]. However in central-plug events, due to the $E_{T}$ of plug electrons being calculated with respect to the nominal $z$ vertex of $0 \mathrm{~cm}$ rather than the true $z$ vertex of the event, the trigger is not fully efficient for plug electrons with $E_{T}<45 \mathrm{GeV}$. This can be seen from figure 3.1 which shows the Z_NOTRACK trigger efficiency as a function of plug electron $E_{T}$ as parameterised in [39]. To combat the reduced efficiency at low $E_{T}$, the ELECTRON_CENTRAL_18 trigger is used as a backup to ensure $\sim 100 \%$ trigger efficiency. Finally the ratio of hadronic energy to electromagnetic energy (had/em) is required to be less than 0.125 in both the Z_NOTRACK and ELECTRON_CENTRAL_18 triggers. This requirement becomes increasingly inefficient as the electron energy increases. Therefore the ELECTRON70_L2_JET trigger, which has a looser had/em requirement, is used as a backup to maintain efficiency at high energies. The ELECTRON_CENTRAL_18 trigger also requires that the electron passes loose $L_{S h r}$ and $C E S \Delta Z$ cuts which respectively ensure the electromagnetic shower shape consistent with that of an electron and that the associated track is well matched. Both these quantities are more precisely defined in section 4.1.4.

\subsection{Monte Carlo Samples}

Monte Carlo generation is performed under the CDF offline software framework with most samples using the PYTHIA event generator [5]. For $Z^{0} / \gamma^{*} \rightarrow e^{+} e^{-}$generation, the 


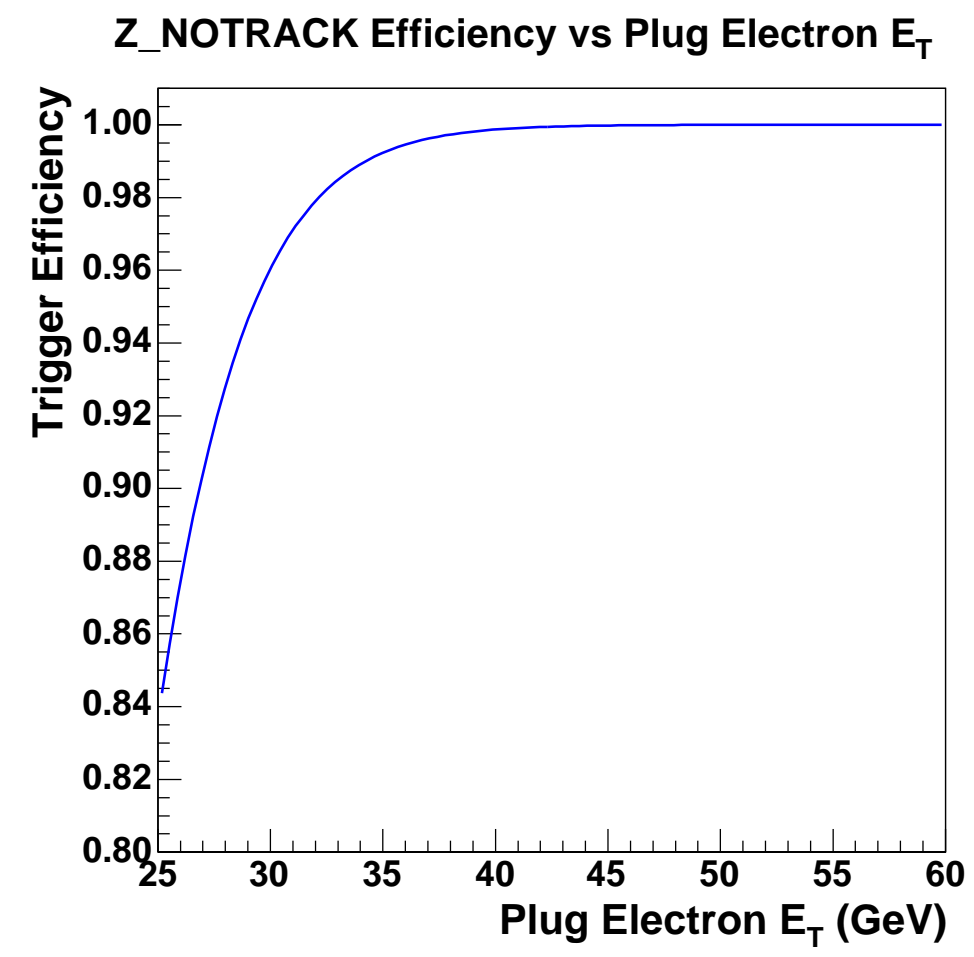

Figure 3.1: The Z_NOTRACK trigger efficiency for central-plug events as a function of plug electron $E_{T}$ as parametrised in [39]. 
parameters which govern the PYTHIA event generator's behaviour have been tuned to match the observed data. Generated events are then passed though a full simulation of the CDF detector which is based on GEANT 3 [41]. The detector configuration is not constant in time due to upgrades and detector subsystem downtime and to correct for this a run dependence is built into the simulation. This principally affects muon and jet reconstruction and has little effect on electron reconstruction, and thus on this analysis.

One of the downsides of high luminosity running conditions is that multiple interactions per bunch crossing become more common. To simulate this, extra minimum bias events are added to Monte Carlo generated events to better match the data. As the number of additional interactions in an event is luminosity dependent, the number of extra minimum bias events is run dependent, with runs which had a higher instantaneous luminosity having correspondingly more minimum bias events added.

The Monte Carlo generated samples that are used for signal and background estimates are listed below. All Monte Carlo generated samples are produced using the CTEQ5L [42] parton distribution functions for the proton and anti-proton. All samples use the PYTHIA event generator although for the $W+$ jet and $W+\gamma$ samples, the generators ALPGEN [43] and WGAMMA [44] are used to generate the matrix elements of the respective processes, with only the hadronisation being performed by PYTHIA. The majority of CDF Monte Carlo generation is done centrally and this analysis uses several of these samples which are listed below under the label "Centrally Generated Samples". For these samples the CDF dataset name is also given for completeness, with the dataset name depending on the physics group who generated it. Monte Carlo samples generated specifically in the course of this analysis are listed under the label "Analysis Specific Generated Samples".

\section{Centrally Generated Samples}

- $Z^{0} \rightarrow e^{+} e^{-}$(zewkae) : A total of 2,255,968 events, generated and processed using the PYTHiA event generator and the offline version 5.3.2 of the CDF software with full run dependence and additional minimum bias overlay.

- $Z \rightarrow \tau^{+} \tau^{-}$(ztop5i) : A total of 1,344,562 events, generated and processed using the PYTHIA event generator and the offline version 5.3.3 of the CDF software with full run dependence applied.

- Di-Boson (wtop1w, wtop1z) : Both samples are generated and processed using the PYTHIA event generator and the offline version 5.3.3 of the CDF software. The wtop1w and wtop1z samples consist of 419,728 and 409,648 events respectively.

- $W+\gamma($ ktop2e, ktop3e) : Both samples are generated with the WGAMMA generator and processed using the PYTHIA event generator and the offline version 5.3.3 of the CDF software. The ktop2e sample consists of $68,273 W^{-} \gamma$ events and the ktop3e sample consists of $102,473 W^{+} \gamma$ events. 
- $W+$ jet (atopaa) : An ALPGEN generated sample consisting of 208,351 events, processed using the PYTHIA event generator and the offline version 5.3.3 of the CDF software.

- $\gamma \gamma$ (pexo4d) : A total of 1,186,952 events generated and processed using the PYTHIA event generator and the offline version 5.3.3 of the CDF software with full run dependence.

\section{Analysis Specific Generated Samples}

- High Mass Drell-Yan : Six samples, each consisting of 50,000 events, are generated with minimum $Z^{0} / \gamma^{*}$ masses of $110,150,200,300,500$ and $800 \mathrm{GeV} / c^{2}$ in order to increase statistics at high invariant mass. This enables a better estimation of the $Z^{0} / \gamma^{*}$ background shape at high invariant mass. The samples are generated in an identical manner to the standard Drell-Yan sample zewkae using the PYTHIA event generator and the offline version 5.3.3 of the CDF software with full run dependence and additional minimum bias overlay.

- $R S$ Graviton $\rightarrow e^{+} e^{-}: 25,000$ events are generated for a series of mass points using the PYTHIA event generator and the offline version 6.1.4 of the CDF software with $k / \bar{M}_{p l}=0.1$ to obtain the signal shape and acceptance for a generic narrow spin-2 particle. The mass points are at $50 \mathrm{GeV} / c^{2}$ intervals starting from $M_{G}=$ 200 and ending at $M_{G}=950 \mathrm{GeV} / c^{2}$. The mass of the Randall-Sundrum graviton is required to be within $10 \%$ of its on-shell mass. This cut is applied by default in the HERWIG [6] event generator which is used by other RS graviton analyses at $\mathrm{CDF}$ [45, 46] and therefore is applied in this analysis in order to be compatible with them.

- $Z^{\prime} \rightarrow e^{+} e^{-}: 10,000$ events are generated for a series of mass points using the PYTHIA event generator and the offline version 5.3.3 of the CDF software with SM-like $Z^{\prime}$ couplings to obtain the signal shape and acceptance for a generic narrow spin- 1 particle. The mass points are at $50 \mathrm{GeV} / c^{2}$ intervals starting from $M_{Z^{\prime}}=200$ and ending at $M_{Z^{\prime}}=950 \mathrm{GeV} / c^{2}$. Additionally the mass of the $Z^{\prime}$ is required to be within $10 \%$ of its on-shell mass. This is done to be consistent with the Randall-Sundrum graviton sample.

All of the samples are processed though the full CDF reconstruction chain and are analysed in an identical manner to the real data. However, in addition, the 'truth' information, such as the four-momenta of the leptons and partons produced in the the hard collision process, is available for Monte Carlo generated samples and this is used to compute acceptances and efficiency corrections. 


\section{Chapter 4}

\section{Electron Identification and Event Selection}

Events are required to pass at least one of the Z_NOTRACK, ELECTRON_CENTRAL_18 or ELECTRON70_L2_JET triggers discussed in section 3.2. Events are then required to have one electron in the central region and another electron in either the central or plug regions, with both electrons passing the identification cuts described in sections 4.1.4 and 4.2.4 appropriate to the classification of the event. Occasionally there are events with more than two electrons passing the identification cuts and this happens 30 times out of a total of 54,367 events. In this case the pair of electrons selected is chosen by the following method:

- if an electron pair's invariant mass is within $10 \mathrm{GeV} / c^{2}$ of the $Z^{0}$ mass of 91.2 $\mathrm{GeV} / c^{2}$, that pair is selected

- if no electron pair's invariant mass is within $10 \mathrm{GeV} / c^{2}$ of the $Z^{0}$ mass, then the electrons with associated tracks are selected, as electrons with tracks are more likely to be genuine electrons than those without associated tracks

- if there are 3 or more electrons with tracks, then the electrons with the two highest energies are selected as higher energy electrons are more likely to be genuine electrons than lower energy electrons

- if there is only one electron with a track, that electron and the highest energy remaining electron are selected 


\subsection{Central Electron Reconstruction and Identifi- cation}

\subsubsection{Central Electron Reconstruction}

Electrons are reconstructed differently depending if they are in the central or the plug calorimeter. In the central calorimeter the reconstruction algorithm first creates a list of the central calorimeter towers, ordered in EM $E_{T}$, with the $E_{T}$ calculated using the nominal $z$ vertex. It then takes the highest $E_{T}$ tower in the list as a seed tower, providing the EM $E_{T}$ is greater than $2 \mathrm{GeV}$. The algorithm then considers the two adjacent towers in $\eta$ in the same $\phi$ wedge. An adjacent tower is added to the cluster if either its EM or hadronic energy is non-zero and is present on the list of towers available for clustering. The electron shower will be almost completely contained within these three towers, with only a small leakage of energy to other nearby towers. This energy is known as the 'leakage energy' [47] and is used to correct the electron isolation defined in section 4.1.4. The cluster is then accepted if the hadronic energy of the cluster is less than $12.5 \%$ of the cluster's EM energy, or if the cluster's EM energy is greater than $100 \mathrm{GeV}$. If the cluster is accepted, then the towers are removed from the list of towers availible for clustering. The algorithm then repeats the process for the next tower in the list.

After the calorimeter clusters have been produced they are matched to CES clusters and COT tracks. The track matching algorithm associates any track which, when extrapolated to the plane of the CES, is within $25 \mathrm{~cm}$ of the shower centre in $x / \phi$ and within $38 \mathrm{~cm}$ of the seed tower centre in $z$. This method can associate multiple tracks with the cluster, which leads to an ambiguity in determining which track resulted from the electron's passage through the COT. This ambiguity is resolved by taking the highest $p_{T}$ track with at least one COT axial hit to be the track left by the electron. The track is also required to be within $5 \mathrm{~cm}$ of the seed tower in the $z$ direction when extrapolated to the plane of the CES. If no track meets these requirements, then there will be no track associated with the cluster as the electron track. The other tracks associated with the cluster are not used by this analysis and therefore in the rest of this document, the term "associated track" will specifically mean the track associated to the cluster as the electron track. The $z$ position at the origin $\left(z_{0}\right)$ of this track is then used for the cluster $z$-vertex and the $E_{T}$ is recalculated. A CES cluster is then seeded using this track and associated to the calorimeter cluster. The track associated with the electron is then refitted using the $2 \mathrm{D}$ position of the beam spot, which is known to an accuracy of about $30 \mu \mathrm{m}$ in $x$ and $y$, as the origin in a process known as "beam-constraining". The track $p_{T}$ is then also corrected for an observed bias in the $E / p$ distribution as a function of $\phi$ in a process known as curvature correction [48]. This well understood bias is the result of wire misalignment within the COT and is applied for data only. 


\begin{tabular}{|c|c|c|c|}
\hline Region & Data (run $\leq 186598)$ & Data (run>186598) & Monte Carlo \\
\hline CEM & $1.000 \pm 0.001$ & $1.003 \pm 0.001$ & $0.9957 \pm 0.0001$ \\
East Plug $|\eta|<1.78$ & $1.019 \pm 0.002$ & $1.023 \pm 0.002$ & $0.9962 \pm 0.0002$ \\
West Plug $|\eta|<1.78$ & $1.016 \pm 0.002$ & $1.025 \pm 0.002$ & $0.9962 \pm 0.0002$ \\
East Plug $|\eta| \geq 1.78$ & $1.009 \pm 0.002$ & $1.009 \pm 0.002$ & $0.9979 \pm 0.0002$ \\
West Plug $|\eta| \geq 1.78$ & $1.004 \pm 0.002$ & $1.008 \pm 0.002$ & $0.9979 \pm 0.0002$ \\
\hline
\end{tabular}

Table 4.1: Energy scale factors for the data and the Monte Carlo simulation. The uncertainties are statistical only.

\subsubsection{Central Electron Energy Scale}

The central electron energy is obtained and corrected using the recommended procedure for CDF analyses which is described [49] and summarised below. The electron energy is defined as the electromagnetic energy of all the towers composing the cluster. The tower response and edge effects are corrected for by applying a position dependent face correction obtained from test beam data [50]. Then the resulting electron energy is scaled so that the mass spectrum around the $Z^{0}$ pole peaks at $91 \mathrm{GeV} / c^{2}$. This is done for both data and Monte Carlo generated events and the energy scaling factors can be found in table 4.1. The motivation for this scaling is to ensure that the energy spectrum of the Monte Carlo simulation agrees with that observed in data.

In order to get the best possible agreement between data and the Monte Carlo simulation, not only is it necessary to ensure that the energy scale is the same but also the energy resolution. If the resolution of the Monte Carlo simulation is better than the data, the energy of electrons in the Monte Carlo simulated events are smeared to decrease the resolution of the simulation to the level observed in data. The smearing process is performed using the following formula

$$
E^{\text {smeared }}=E \cdot \mathbf{G}(\text { smear })
$$

where $\mathbf{G}$ (smear) is a random number generated from a Gaussian with $\mu=1$ and $\sigma$ is the fraction by which to smear the energy. The amount of smearing is varied to find the value which gives the minimum $\chi^{2}$ between the mass spectrum measured in the data and the mass spectrum predicted by the Monte Carlo simulation. In the case for the CEM, the resolution of the Monte Carlo simulation is actually slightly worse than the data and therefore no smearing is required to further decrease the resolution of the Monte Carlo simulation. Figure 4.1 shows a comparison of the data and the Monte Carlo predicted mass spectrum in the central-central channel at the $Z^{0}$ pole after the scaling to $91 \mathrm{GeV} / c^{2}$. The two mass spectra agree well, giving confidence in the Monte Carlo simulation. 


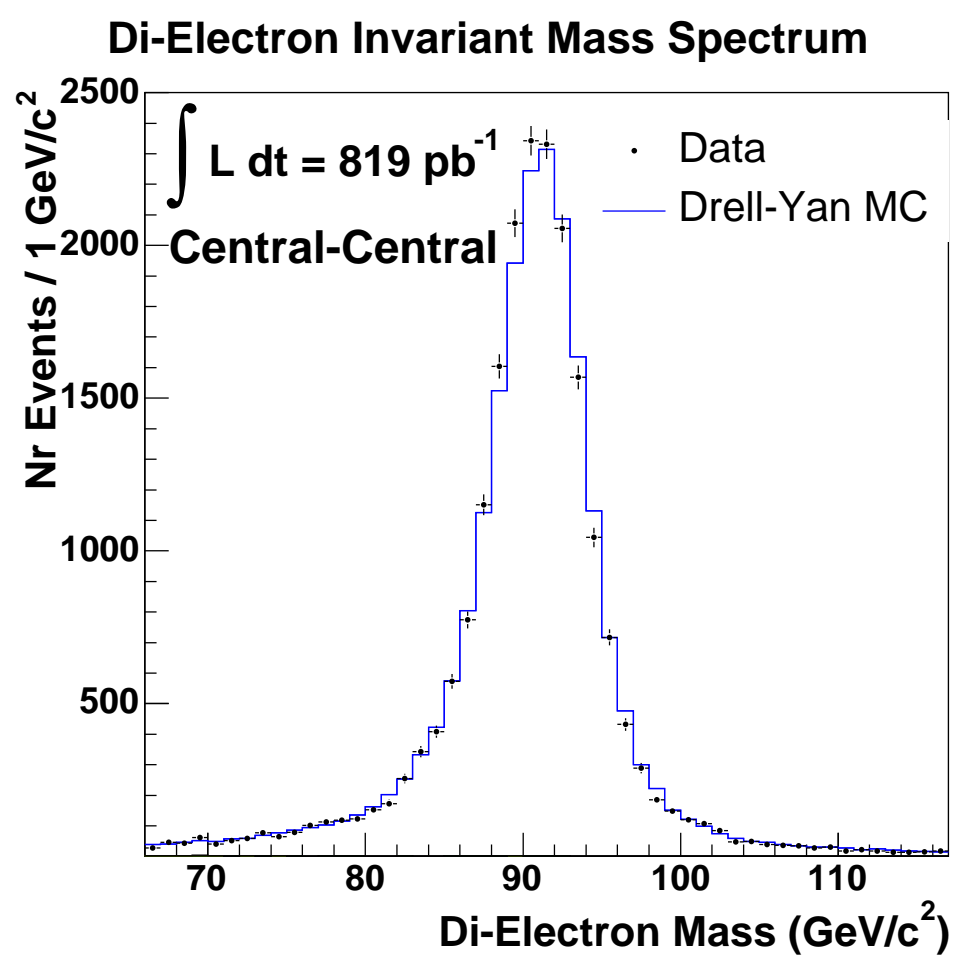

Figure 4.1: The measured di-electron mass spectrum in the central-central channel in the region of the $Z^{0}$ pole together with the prediction from Monte Carlo simulation. 


\section{Uncertainties on the Monte Carlo CEM Energy Scale and Resolution}

As this analysis is essentially a comparison between the observed data and the prediction from the Monte Carlo simulation, the analysis is more sensitive to the difference between the data and Monte Carlo simulation energy scales rather than the absolute energy scale. Therefore the uncertainty on the Monte Carlo simulation energy scale is derived from the differences between the energy scales of the data and the Monte Carlo simulation. These differences could either arise from local differences between the two energy scales in individual sections of the calorimeter or from the energy scales of the data and the Monte Carlo simulation evolving differently as a function of energy.

To investigate the extent of any local differences between the energy scales of the data and the Monte Carlo simulation, the CEM is divided up into sub-regions in $\phi$ and $\eta$, with each sub-region roughly corresponding to a calorimeter tower. The percentage difference between the means of the data and Monte Carlo simulated mass spectra in the range of $82-98 \mathrm{GeV} / c^{2}$ is then calculated for each of the sub-regions. The percentage differences, together with a Gaussian fit, are shown in figure 4.2. To investigate the extent of any differences between the data and Monte Carlo simulation energy scales as function of energy, events are organised into bins of electron energy. A Gaussian is fitted to the mass spectrum of each bin in the range of $86-98 \mathrm{GeV} / c^{2}$. For each bin, the percentage difference between the Gaussian means of the data and Monte Carlo simulated mass spectra is calculated and these are collectively displayed in figure 4.3. The deviations between the energy scales of the data and the Monte Carlo simulation are within $1 \%$ in both studies, and therefore a $1 \%$ systematic uncertainity is quoted on the Monte Carlo CEM energy scale.

For the energy resolution, the uncertainty is evaluated following the method in [9]. The uncertainty on the resolution is therefore taken to be the extra smearing required to increase the $\chi^{2}$ between the data and the Monte Carlo simulated mass spectra from its minimum value by 9 . This corresponds to the $3 \sigma$ bound on the estimated additional smearing and is chosen to be a conservative estimate of the uncertainty. An increase in the $\chi^{2}$ by 9 is found to correspond to an increase in the smearing of $0.7 \%$ for the CEM.

\subsubsection{Tower 9 Events}

A special case in electron reconstruction occurs when the electron is incident in a tower on the edge of the central calorimeter. These towers are collectively known as 'tower 9'. Since on one side there is no adjacent tower, if the electron is incident near the edge of that side of the tower, part of the resulting electromagnetic shower can be lost. This could potentially lead to a poor measurement of the electron's energy. Electrons in tower 9 are typically rejected by CDF analyses for this reason but by retaining these events, the total acceptance can be increased by about 2-3\%. The face correction from section 4.1.2 should adequately correct for the energy mismeasurement. To determine how well this works in practice, the mass spectrum at the $Z^{0}$ pole for events 


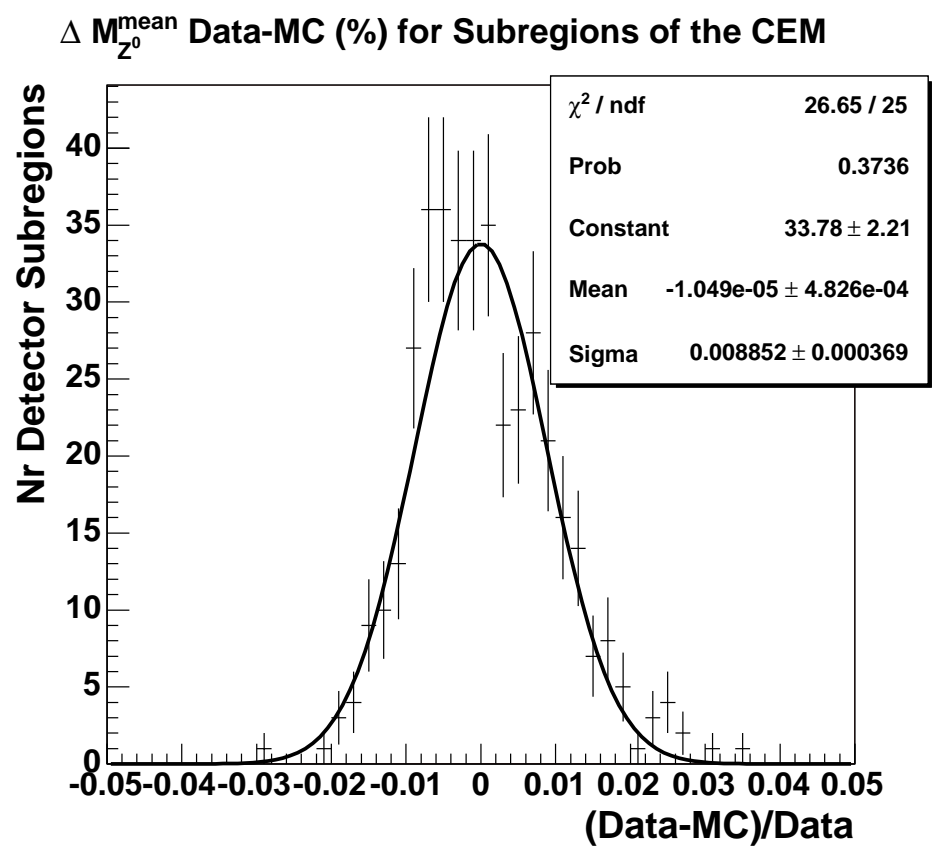

Figure 4.2: The difference of the means of the data and Monte Carlo simulated mass spectra between $82-98 \mathrm{GeV} / c^{2}$ for various bins of eta and phi for central-central events. A Gaussian is fitted to observed differences and the fit $\chi^{2}$ and probability, as well as the mean, sigma and normalisation constant, are shown in the insert. 


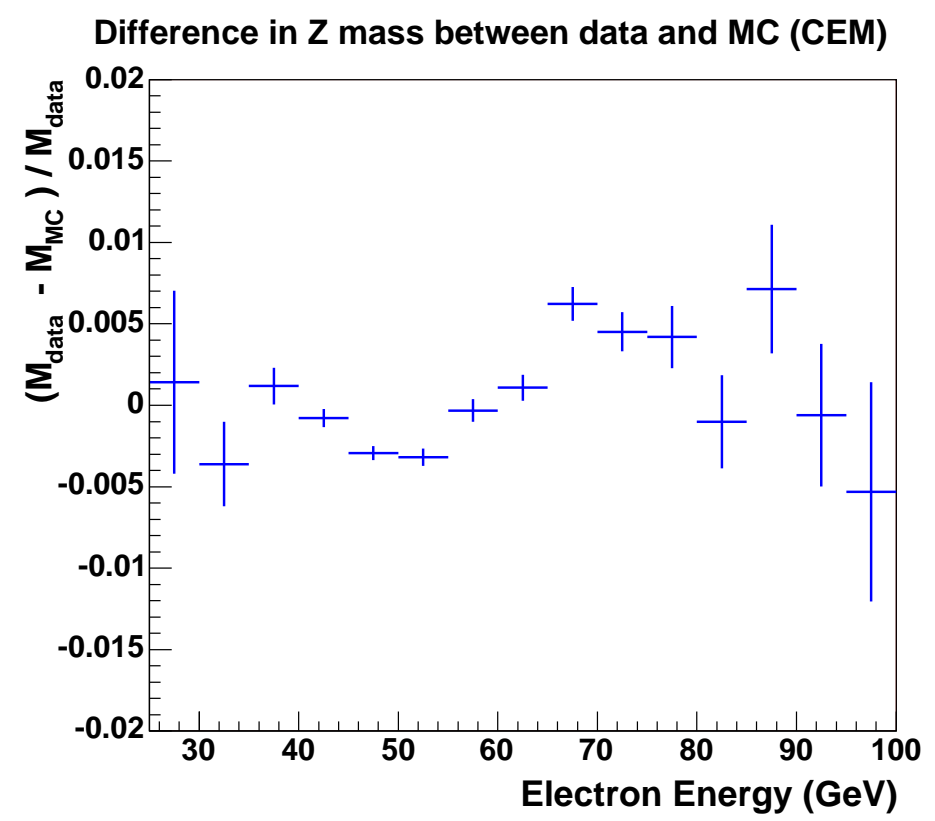

Figure 4.3: The difference of the Gaussian means of the data and Monte Carlo simulated mass spectra, fitted in the range of $86-98 \mathrm{GeV} / c^{2}$, as a function of electron energy for central-central events.

which pass all the standard selection cuts and have an electron fiducial in tower 9 is investigated. Figure 4.4 shows this mass distribution, fitted with a Gaussian between $86<M_{e e}<98 \mathrm{GeV} / c^{2}$. For comparison purposes the mass spectrum for events fiducial in tower 5, a typical tower, is also shown. The data peak approximately at $91 \mathrm{GeV} / c^{2}$, showing that the face correction adequately takes the energy losses into account. However in the Monte Carlo generated events, the mass peak is lower by about $5 \%$ and the resolution is worse. The difference in the mass peak translates to a $11.7 \%$ difference in the energy scale. As a result, the energy of tower 9 electrons in Monte Carlo generated events are scaled up by $11.7 \%$ to better match the data. When this additional scaling is applied, the width of a Gaussian fitted to the Monte Carlo predicted mass spectrum is found to be $5.2 \pm 0.2 \mathrm{GeV} / c^{2}$, while the corresponding width for data is $3.7 \pm 0.2 \mathrm{GeV} / c^{2}$. The decreased resolution of the Monte Carlo simulation in tower 9 will cause the estimated $Z^{0} / \gamma^{*} \rightarrow e^{+} e^{-}$distribution to deviate from true distribution of the data. To evalute this effect, the resolution of another tower in the Monte Carlo simulation is decreased by the amount the resolution of tower 9 in the Monte Carlo simulation is lower than the data and the change in the predicted mass spectrum is observed. Tower 8 is chosen for this due to its proximity to tower 9 . The resulting difference in the mass spectrum at high mass is found to be negligible compared to other uncertainties. 


\section{Central-Central Mass Spectrum for tower 9 Events}

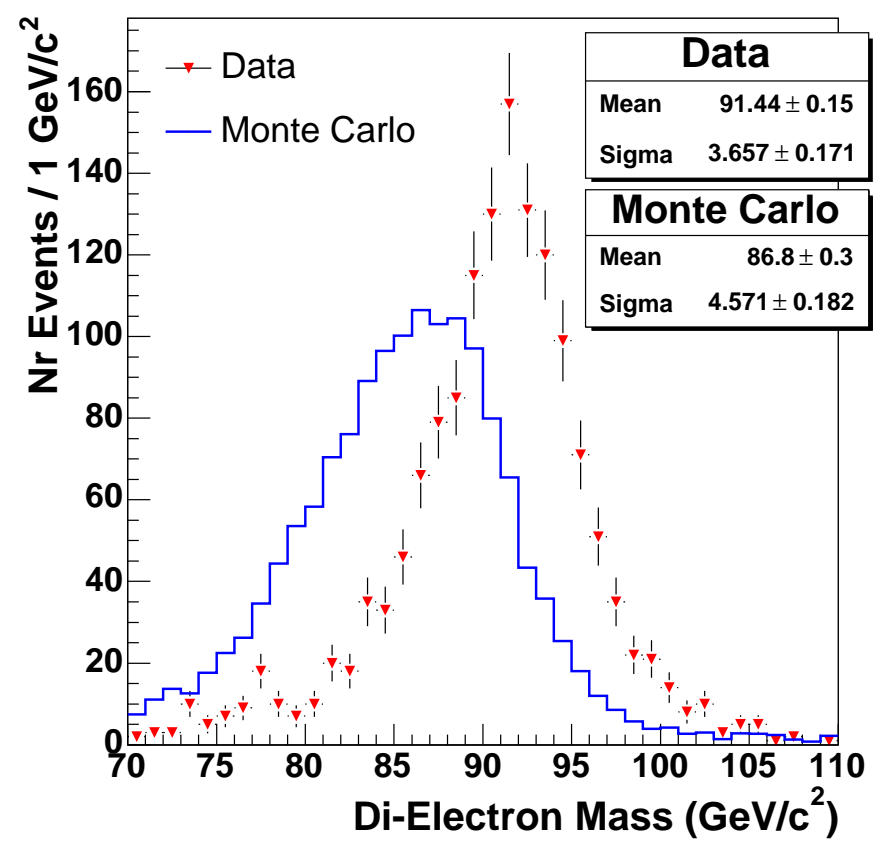

Central-Central Mass Spectrum for tower 5 Events

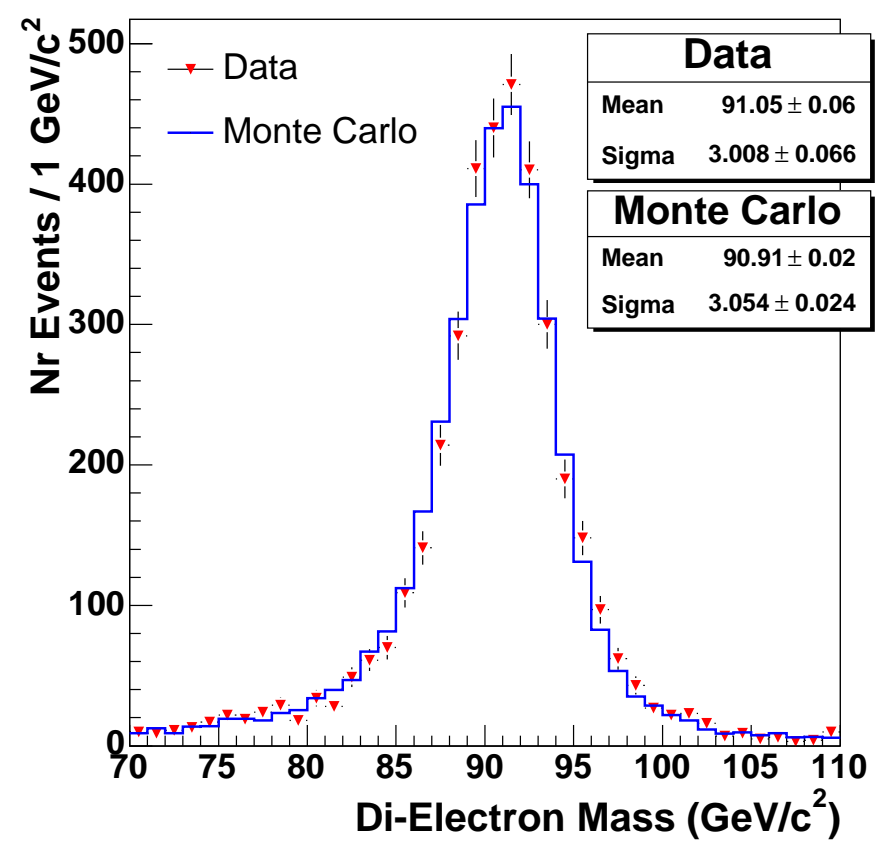

Figure 4.4: The mass spectrum for central-central events with at least one electron in tower 9 is shown in the upper plot. While the Gaussian fit is performed in the standard range $86-98 \mathrm{GeV} / c^{2}$ for data, the fit range for Monte Carlo generated mass spectrum is 81-93 GeV $/ c^{2}$, due to the lower peak position. The corresponding mass spectrum for tower 5, a typical tower, is shown in the lower plot for comparison purposes. 


\subsubsection{Central Electron Identification}

The electron candidate is required to pass the identification cuts in table 4.2 appropriate to its partner's region. Central electrons whose partner is another central electron are required to pass the CEM CC cuts and those whose partner is a plug electron are required to pass the CEM CP cuts. The identification variables used in table 4.2. along with other key electron variables, are defined below. Details on how these cuts were chosen can be found in appendix A.

The central electron identification variables used in this analysis are:

- E : Electron Energy

The electromagnetic energy of all the towers composing the cluster. The energy is corrected as discussed in section 4.1.2.

- $E_{T}$ : Electron Transverse Energy

The electron energy multiplied by $\sin \left(\theta_{t r k}\right)$, where $\theta_{t r k}$ is the polar angle of the highest $p_{T}$ beam-constrained track associated with the cluster.

- Track $p_{T}$ : Track Transverse Momentum

The beam-constrained and curvature corrected $p_{T}$ of the highest $p_{T}$ track associated with the cluster.

- Track $z_{0}$ : Z Position of the Track Origin

The $z_{0}$ of the highest $p_{T}$ beam-constrained track associated with the cluster.

- $\mathrm{Had} / \mathrm{em}$

The ratio of the total hadronic to total electromagnetic energy of all the towers composing the cluster.

- Isolation $E_{T}$

The total hadronic and electromagnetic transverse energy surrounding the electron in the calorimeter in a cone of radius $\Delta R=0.4$ corrected for the leakage of the energy of the electron outside of its calorimeter cluster. The isolation $E_{T}$ is also corrected for the effects of additional minimum bias events leading to multiple interactions in the event by subtracting $0.35 \mathrm{GeV}$ or $0.27 \mathrm{GeV}$ per additional vertex for data and Monte Carlo respectively, as recommended by the CDF Joint Physics group [51.

\section{- Isolation $E_{T}^{\text {corr }}$}

The isolation $E_{T}$ minus 0.02 times the electron $E_{T}$. This variable accounts for fact that the typical isolation $E_{T}$ of an electron increases with increasing electron $E_{T}$ and is defined to simplify notation for some tables and figures.

\section{- $L_{S h r}$ : Lateral Shower Sharing Variable}

A measure of how well the energy deposits in the adjacent towers in $\eta$ matches 
that expected for an electromagnetic shower [52]. As a typical cluster in the CEM has two adjacent towers to the seed tower in the $\phi$ wedge, $L_{S h r}$ is a two tower sum. For towers 0 and 9 , which are at the end of the wedge and only have one adjacent tower, $L_{S h r}$ is only calculated over one tower. $L_{S h r}$ is defined as

$$
L_{S h r}=0.14 \frac{\sum_{i}\left(E_{i}^{\text {meas }}-E_{i}^{\text {pred }}\right)}{\sqrt{\left(0.014 \sqrt{E_{E M}}\right)^{2}+\sum_{i}\left(\Delta E_{i}^{\text {pred }}\right)^{2}}}
$$

where $E_{i}^{\text {meas }}$ is the measured energy in the adjacent tower, $E_{i}^{\text {pred }}$ is the predicted energy from test beam data in the adjacent tower and is a function of the local $z$ coordinate in the calorimeter, $\Delta E_{i}^{\text {pred }}$ is the uncertainty on the predicted energy in the adjacent tower and $E_{E M}$ is the energy of the electron. The sum is over all adjacent towers in the same wedge and all energies are in units of $\mathrm{GeV}$. The $z_{0}$ of highest $p_{T}$ beam-constrained track associated with the cluster is used for the primary vertex and is extrapolated to the CES plane to give the local $z$ coordinate in the calorimeter.

- $E / p$

The transverse energy of the electron divided by the track $p_{T}$.

- CES $\triangle X$

The difference between the local CES $x$ position of the highest $p_{T}$ beam-constrained track, when extrapolated to the CES plane, and the $x$ position of the electromagnetic shower as measured by the CES.

\section{- $\mathrm{CES} \triangle Z$}

The difference between the local CES $z$ position of the highest $p_{T}$ beam-constrained track, when extrapolated to the CES plane, and the $z$ position of the electromagnetic shower as measured by the CES.

\section{- Fiducial}

To reject events in inactive detector regions, the electromagnetic shower is required to be in the region

$$
\begin{array}{r}
-21 \mathrm{~cm} \leq X_{C E S} \leq 21 \mathrm{~cm} \\
9 \mathrm{~cm} \leq Z_{C E S} \leq 230 \mathrm{~cm}
\end{array}
$$

where $X_{C E S}$ and $Z_{C E S}$ are the $x$ and $z$ positions of electromagnetic shower in the CES local coordinate system as measured by the CES. In addition, the electron is required to not be fiducial in the uninstrumented 'chimney' wedge in tower 7. Various fiducial codes are used for central electrons at CDF. The codes of 1 or 2 correspond to the electron satisfying the above requirements, with a code of 1 further indicating that the electron is not in tower 9 and a code of 2 indicating that the electron is in tower 9 . Other codes indicate that the electron has not satisfied all of the above requirements. 


\begin{tabular}{|c|c|c|}
\hline Variable & CEM CC (CEMCC) & CEM CP (CEMCP) \\
\hline Region & FEM CEM & $=\mathrm{CEM}$ \\
Fiducial & Fid $=1$ or 2 & Fid $=1 \mathrm{or} 2$ \\
$\mathrm{E}_{T}$ & $\geq 25 \mathrm{GeV}$ & $\geq 25 \mathrm{GeV}$ \\
Track $z_{0}$ & $\leq 60 \mathrm{~cm}$ & $\leq 60 \mathrm{~cm}$ \\
Track $p_{T}\left(E_{T}<100\right)$ & $\geq 15 \mathrm{GeV} / \mathrm{c}$ & $\geq 15 \mathrm{GeV} / \mathrm{c}$ \\
Track $p_{T}\left(E_{T} \geq 100\right)$ & $\geq 25 \mathrm{GeV} / \mathrm{c}$ & $\geq 25 \mathrm{GeV} / \mathrm{c}$ \\
Had $/ \mathrm{em}$ & $\leq 0.055+0.00045 \times E$ & $\leq 0.055+0.00045 \times E$ \\
Isolation $E_{T}$ & $\leq 3+0.02 \times E_{T} \mathrm{GeV}$ & $\leq 3.0+0.02 \times E_{T} \mathrm{GeV}$ \\
$L_{S h r}$ & $\leq 0.2$ & $\leq 0.2$ \\
$E / P\left(E_{T}<100\right)$ & $\leq 2.5+0.015 \times E_{T} \mathrm{GeV}$ & $\leq 2.5+0.015 \times E_{T} \mathrm{GeV}$ \\
CES $\Delta Z$ & $\leq 5.0 \mathrm{~cm}$ & $\leq 5.0 \mathrm{~cm}$ \\
CES $\Delta X$ & $\leq 3.0 \mathrm{~cm}$ & $\leq 3.0 \mathrm{~cm}$ \\
Conversion & - & false \\
\hline \multicolumn{2}{|c}{}
\end{tabular}

Table 4.2: The CEM electron selection cuts. All cuts are performed with $E$ in units of $\mathrm{GeV}$.

\section{- Conversion}

Events where a photon has converted into an $e^{+} e^{-}$pair are rejected using the standard CDF conversion finding algorithm. The algorithm identifies a pair of tracks as a conversion candidate if they are of opposite sign and satisfy

$$
\left|\delta_{x y}\right|<0.2 \mathrm{~cm} \text { and }\left|\Delta \cot \theta_{\text {trk }}\right|<0.04
$$

where $\delta_{x y}$ is the distance of the closest approach of the two tracks. If the electron track is found to be one of a pair of tracks identified as a conversion candidate, the electron is marked as a conversion unless there is a third track which can form a conversion candidate pair with either of the previous two tracks. In this case, the event is classified as a 'trident' event and is accepted in this analysis. Trident events are genuine electrons which have radiated a hard photon which then subsequently pair converts to give a possibility of three tracks in close proximity.

\subsection{Plug Electron Reconstruction and Identifica- tion}

\subsubsection{Plug Electron Reconstruction}

Electron reconstruction in the plug calorimeter is more complicated than in the central calorimeter as the shower can spread to neighbouring towers in $\phi$ as well as $\eta$ due to the design and geometry of the plug calorimeter. The $2 \times 2$ reconstruction algorithm 
attempts to make clusters of 2 towers in $\eta$ by 2 towers in $\phi$, to give a total of four towers per cluster arranged in a square shape. It achieves this by first creating a list of clusterable 1 plug calorimeter towers, ordered in EM $E_{T}$, with the $E_{T}$ calculated using the nominal $z$ vertex. It then takes the highest $E_{T}$ tower in the list as a seed tower, provided the EM $E_{T}$ is greater than $2 \mathrm{GeV}$. Next it takes the highest EM $E_{T}$ tower, with which the seed tower shares either a border or a corner with, and adds it to the cluster as a daughter tower if the tower's EM $E_{T}$ is greater than $100 \mathrm{MeV}$. To finish the cluster, the algorithm then searches for the highest EM $E_{T}$ pair of towers which would complete the $2 \times 2$ cluster and adds them if the pair's EM $E_{T}$ is greater than $100 \mathrm{MeV}$. The algorithm accepts the cluster if the hadronic energy of the cluster is less than $12.5 \%$ of the cluster's EM energy, or the cluster's EM energy is greater than $100 \mathrm{GeV}$. If the cluster is accepted then the towers are removed from the list of clusterable towers and the algorthim repeats the process for the next tower in the list.

If the electron is incident near the centre of the seed tower, the shower may not be fully contained in the $2 \times 2$ cluster as the electron will be nearer the edge of the cluster. Similarly to CEM electrons, the energy the electron deposits outside the $2 \times 2$ cluster is known as the leakage energy. The average leakage energy as a function of position in the $2 \times 2$ cluster, measured in [47], is used to estimate the leakage energy for an individual electron. Due to the design and geometry of the plug calorimeter, the typical leakage energy of an electron is much larger in the PEM than the CEM and therefore for PEM electrons it is used to correct the electron energy as well as the amount of energy surrounding the electron in the calorimeter.

Due to the acceptance of the COT, the tracking efficiency is poor in the plug and a track is not required to be associated with the cluster. Angular information is instead obtained using the PES, with the PES $\eta_{\text {det }}$ converted to $\eta_{\text {event }}$ using the $z_{0}$ of the central electron. To this end, plug electrons are required to have an associated PES cluster. Tracking information can be obtained using the 'Phoenix tracking' algorithm discussed in section 4.2.3, albeit with a large decrease in acceptance.

\subsubsection{Plug Energy Scale}

Like the central electron energy, the plug electron energy is obtained and corrected using the recommended procedure for CDF analyses [49]. The first step of this procedure is to obtain the total electromagnetic energy of all the towers composing the $2 \times 2$ cluster. This energy is then corrected for tower response and edge effects using a position dependent face correction [53]. Next the leakage energy is added to account for the electromagnetic shower not fully being contained in the $2 \times 2$ cluster. Then the energy measured in the Plug Pre-Radiator is added to obtain the total energy deposited by the electron. Finally the energy is scaled using the scale factors in table 4.1 so that for data and Monte Carlo events the $Z^{0}$ peak is at $91 \mathrm{GeV} / c^{2}$.

\footnotetext{
${ }^{1}$ not all plug calorimeter towers are clusterable, the most forward $\eta$ tower is not due to its high activity resulting from its proximity to the beam pipe.
} 
The resolution of the Monte Carlo simulation is adjusted to resolution measured in the data in an identical manor to that used for CEM electrons in section 4.1.2. To obtain the minimum $\chi^{2}$ between the central-plug data and Monte Carlo simulated mass spectra, an additional $1.7 \%$ smearing is applied to the energy of PEM electrons in the Monte Carlo simulation. Figure 4.5 shows a comparision of the data and the Monte Carlo predicted mass spectrum in the central-plug channel at the $Z^{0}$ pole after the scaling to $91 \mathrm{GeV} / c^{2}$. Again there is good agreement between the data and the Monte Carlo simulation.

\section{Uncertainties on the Monte Carlo CEM Energy Scale and Resolution}

The uncertainties on the PEM energy scale and resolution are evaluated using the same methods used to evaluate the uncertainties on the CEM energy scale and resolution in section 4.1.2. Figure 4.6 shows the percentage differences between the means of the mass spectra of data and Monte Carlo simulation over the various sub-regions of the PEM. Figure 4.7 shows the percentage differences between the means of the mass spectra of data and Monte Carlo simulation as a function of energy. In both figures, a $1 \%$ difference adequately covers the deviations between the data and Monte Carlo energy scales and therefore a $1 \%$ systematic uncertainty is taken on the Monte Carlo PEM energy scale. The uncertainty on the energy resolution for the Monte Carlo in the PEM is estimated using the $\chi^{2}+9$ method, and is found to be $0.5 \%$.

\subsubsection{Phoenix Tracking}

To obtain tracking information in the plug, the Phoenix (PHX) tracking algorithm [54] is used. This algorithm constructs a hypothetical track for the electron between the beam spot and the position of the electromagnetic shower in the calorimeter, using the measured electron energy to set the track momentum. This is sufficient to define the track up to a sign ambiguity. The algorithm then takes the two resulting hypothetical tracks and fits them to the silicon hits recorded in the silicon tracker. Of the two fitted tracks, the one with the lowest $\chi^{2} / n_{\text {dof }}$ resulting from the fit to the silicon hits is accepted as the electron track. Electrons required to have a Phoenix track are referred to as Phoenix electrons. The Phoenix tracking efficiency is about $85 \%$ for $1.2 \leq \eta \leq 2.0$, but falls off sharply above $\eta \sim 2.0$ [55]. For this reason, when a Phoenix track is required, the allowed $\eta$ range of the plug electron is restricted to $\eta \leq 2$. Furthermore it requires that the run be marked 'good' for silicon, which reduces the available data by about $9 \%$. However requiring a Phoenix track reduces the centralplug background levels to that of the central-central channel. The study described in appendix $\mathrm{A}$ concludes that the reduced selection efficiency is not offset by the improved background reduction. Therefore a plug electron is not required to have an assoicated Phoenix track in this analysis except for some studies, such as efficiency studies, where having a small pure sample is preferable to a larger less pure sample. 


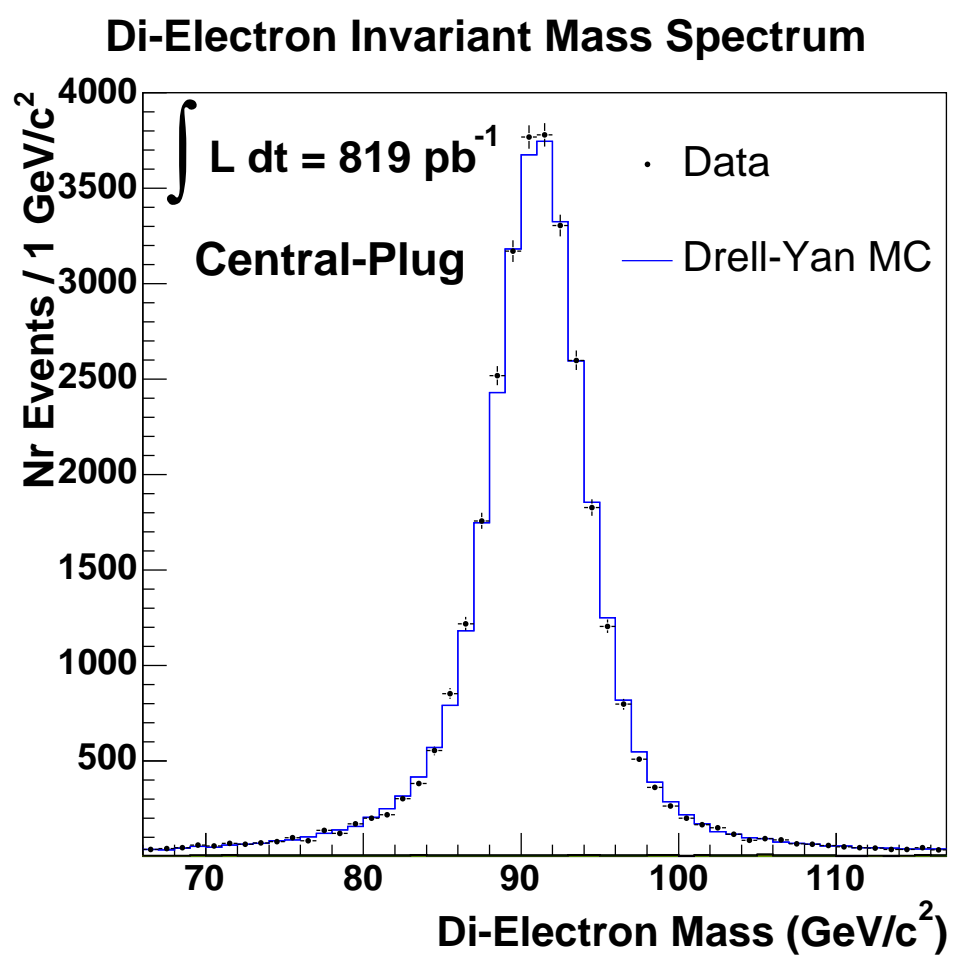

Figure 4.5: The measured di-electron mass spectrum in the central-plug channel in the region of the $Z^{0}$ pole together with the prediction from Monte Carlo simulation. 


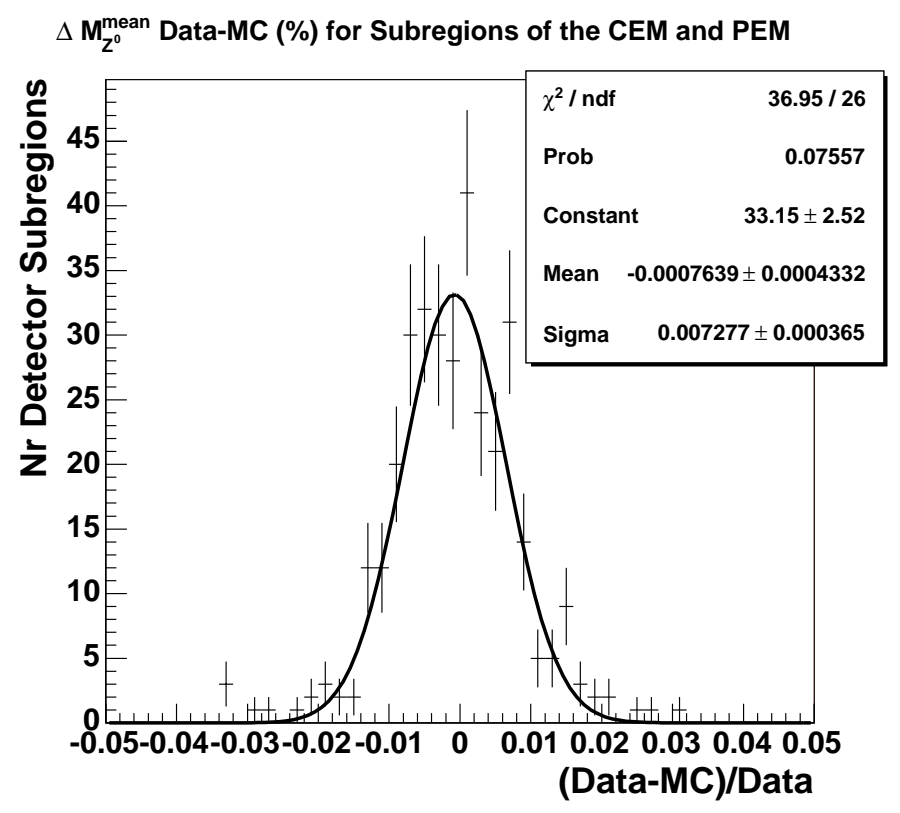

Figure 4.6: The difference of the means of the data and Monte Carlo simulated mass spectra between $82-98 \mathrm{GeV} / c^{2}$ for various bins of eta and phi for central-plug events. A Gaussian is fitted to observed differences and the fit $\chi^{2}$ and probability, as well as the mean, sigma and normalisation constant, are shown in the insert. 


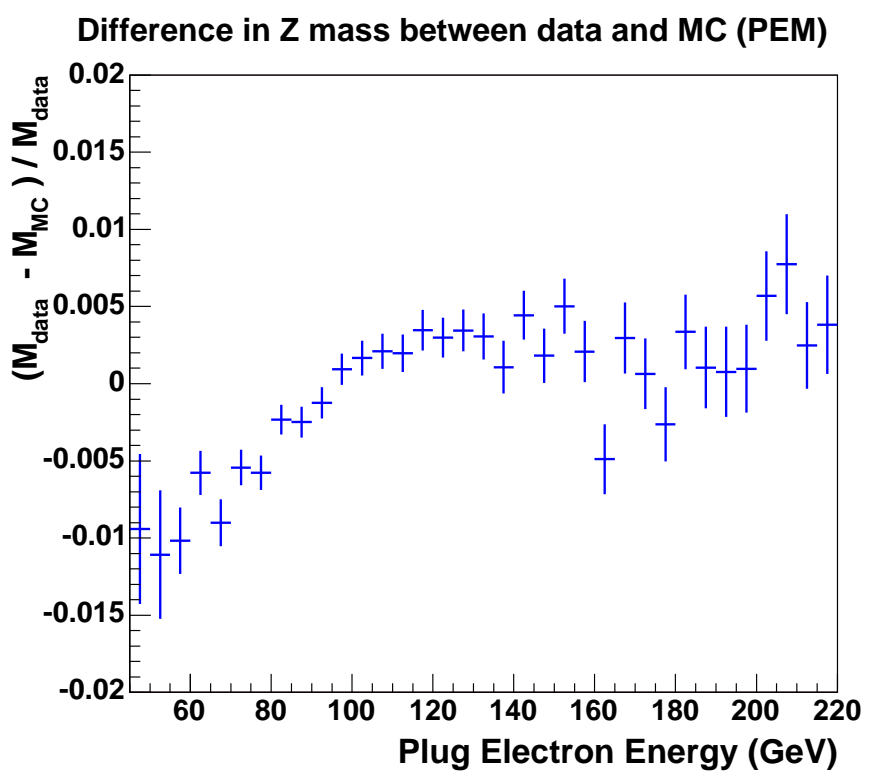

Figure 4.7: The difference of the Gaussian means of the data and Monte Carlo simulated mass spectra, fitted in the range of $86-98 \mathrm{GeV} / c^{2}$, as a function of electron energy for central-plug events. For low energies, increased background fractions lead the Monte Carlo simulation slightly overestimating the mass scale.

\subsubsection{Plug Electron Identifications Variables}

The electron candidate is required to pass the selection cuts of table 4.3. The identification variables used in table 4.3. along with other key electron variables, are defined below. Details on how these cuts were chosen can be found in appendix A.

The plug electron identification variables used in this analysis are:

- $E$ : Electron Energy

The electromagnetic energy of all the towers composing the cluster produced using the $2 \times 2$ clustering algorithm. The cluster EM energy is corrected as discussed in section 4.2.2.

- $E_{T}$ : Electron Transverse Energy

The electron energy multiplied by $\sin \left(\theta_{P E S}\right)$ where $\theta_{P E S}$ is the polar angle of the electron calculated from the PES measured $\eta$ using the $z_{0}$ of the central electron to convert it from a detector based quantity to an event based quantity.

- $\mathrm{Had} / \mathrm{em}$

The ratio of the total hadronic to total electromagnetic energy of all the towers composing the cluster. 
- Isolation $E_{T}$

e total hadronic and electromagnetic transverse energy surrounding the electron in the calorimeter in a cone of radius $\Delta R=0.4$ corrected for the leakage of the energy of the electron outside of its calorimeter cluster. The isolation $E_{T}$ is also corrected for the effects of additional minimum bias events leading to multiple interactions in the event by subtracting $0.35 \mathrm{GeV}$ or $0.27 \mathrm{GeV}$ per additional vertex for data and Monte Carlo respectively, as recommended by the CDF Joint Physics group.

- Isolation $E_{T}^{c o r r}$

The isolation $E_{T}$ minus 0.02 times the electron $E_{T}$. This variable accounts for fact that the isolation $E_{T}$ increases with increasing electron $E_{T}$ and is defined to simplify notation for some tables and figures.

- PES $\eta$

The detector $\eta$ of the electromagnetic shower as measured by the PES.

- PEM $\chi_{3 \times 3}^{2}$

The $\chi^{2}$ obtained from comparing the distribution of energy deposited by the electron in the $3 \times 3$ block of towers surrounding the seed tower to the distribution of energy deposits in those towers measured using test beam data.

- $N_{\text {hits }}^{S i}$

The number of silicon hits associated with the Phoenix track. This is only defined for Phoenix electrons.

- Track $z_{0}$

The $z_{0}$ of the Phoenix track associated with the electron. This is only defined for Phoenix electrons. 


\begin{tabular}{|c|c|}
\hline Variable & PEM (PEM) \\
\hline Region & $=\mathrm{PEM}$ \\
$\mathrm{E}_{T}$ & $\geq 25 \mathrm{GeV}$ \\
$\mathrm{PES} \eta$ & $\leq|.2 \leq| \leq 3.0$ \\
$\mathrm{Had} / \mathrm{em}$ & $\leq 0.05$ for $E \leq 100 \mathrm{GeV}$ \\
$\mathrm{PEM} \chi_{3 \times 3}^{2}$ & $\leq 1.6+0.02 \times E_{T} \mathrm{GeV}$ \\
Isolation $E_{T}$ & $\leq 60 \mathrm{~cm}$ \\
\hline Track $z_{0}$ & $\geq 3$ \\
$N_{\text {hits }}^{S i}$ & \\
\hline
\end{tabular}

Table 4.3: The PEM electron selection cuts. The Had/em cut is performed with $E$ in units of GeV. The Track $z_{0}$ and $N_{\text {hits }}^{S i}$ cuts are only used when an associated Phoenix track is required. 


\section{Chapter 5}

\section{Electron Identification Efficiency and Acceptance}

The total selection efficiency is separated into four parts: the kinematical and geometrical acceptance, the identification efficiency, the $z$-vertex cut efficiency and the trigger efficiency. This is necessary as each part requires a different method to measure it.

The kinematic and geometrical acceptance is estimated using Monte Carlo simulation as it is not possible to measure the acceptance using data events. The identification efficiency at high electron energies $\left(E_{T} \geq 100 \mathrm{GeV}\right)$ is also estimated using Monte Carlo simulation due to the lack of a sample of high energy electrons in the data with a sufficient purity to perform a reliable efficiency measurement on. However the $Z^{0}$ pole proves a pure enough electron sample with which to measure the efficiency in the data at lower energies. This is used to correct the identification efficiency estimated by the Monte Carlo simulation by comparing the measured identification efficiency of data and Monte Carlo simulated electrons at the $Z^{0}$ pole. This is necessary as while the kinematic and geometric distributions affecting the acceptance are relatively easy to model, the identification variables are much more difficult for the Monte Carlo simulation to correctly describe and, as such, the simulation of the identification variables is imperfect.

The efficiency of the $z$-vertex cut is measured using minimum bias events and the Tevatron $p \bar{p}$ longitudinal beam profile and has been found to be $0.951 \pm 0.003$ [56]. The trigger efficiency is $100 \%$ due to the use of the two additional backup triggers discussed in section 3.2.

\subsection{Electron Identification Efficiency}

Electron identification efficiencies are estimated using Monte Carlo generated events for a given $e^{+} e^{-}$invariant mass point and then subsequently corrected by a scale factor obtained by comparing the measured efficiencies for data and Monte Carlo generated events at the $Z^{0}$ pole. The efficiencies are measured following the method described in [55, 57]. This method is also briefly summarised in section 5.1.1, The identification 
efficiencies as a function of the $e^{+} e^{-}$invariant mass are displayed in figure 5.1. As well as measuring the total efficiency, it can be informative to look at the efficiencies of the individual identification cuts. This is done by measuring the ' $\mathrm{N}-1$ ' efficiency of an identification cut. The N-1 efficiency is defined as the efficiency of the cut on a sample which has already passed all the other cuts. This allows the effect of each cut on the sample to be determined although if two variables are correlated, it will give each of them an artificially high efficiency. As the N-1 efficiencies are only shown to give an idea of how efficient an individual cut is, they are only shown in this chapter at an energy scale around the $Z^{0}$ pole for brevity. Appendix A shows all the individual N-1 efficiencies vs electron $E_{T}$ as well as the background rejection of each cut.

\subsubsection{Method used to Measure the Electron ID Efficiency}

To measure the central electron identification efficiencies, events with two electrons are selected with one electron required to pass the relevant CEM cuts of table 4.2 and the other required to pass the CEM probe cuts of table 5.1. In addition, the two electrons are required to have opposite charge. For studies at the $Z^{0}$ pole, the invariant mass of the two electrons is required to be in range of $76<M_{e e}<106 \mathrm{GeV} / c^{2}$. When measuring the plug electron identification efficiency, events are required to have one central electron passing the extra tight cuts of table 5.1 and one plug electron passing the PEM probe cuts of table 5.2. The plug electron is required to have an associated Phoenix track in order to reduce the number of background events present in the sample. For studies at the $Z^{0}$ pole, the invariant mass of the two electrons is required to be within the range of $81<M_{e e}<101 \mathrm{GeV} / c^{2}$, with the smaller mass range chosen due to the increased background in this channel. Any event with more than one di-electron pair satisfying these selection requirements is rejected. The identification efficiencies in the central region are then calculated using the formula

$$
\epsilon_{C E M}=\frac{2 \times N_{C E M-C E M}}{N_{C E M-C E M}+N_{C E M-P C E M}}
$$

where $N_{C E M-C E M}$ is the number of events with both electrons passing all the normal CEM cuts and $N_{C E M-P C E M}$ is the number of events with one electron passing all the normal CEM cuts and one electron passing all probe cuts. For plug electrons, the formula to calculate the identification efficiency is simpler as one electron of the dielectron pair did not already pass the PEM cuts when constructing the sample and so the formula becomes

$$
\epsilon_{P E M}=\frac{N_{T C E M-P E M}}{N_{T C E M-P P E M}}
$$

where $\mathrm{N}$ is the number of events with the index TCEM indicating the central electron has passed all the central extra tight cuts, the index PEM indicating the plug electron has passed all the normal plug cuts and the index PPEM indicating the plug electron 


\begin{tabular}{|c|c|c|}
\hline Variable & CEM Probe (PCEM) & CEM Extra Tight (TCEM) \\
\hline Region & $=\mathrm{CEM}$ & $=\mathrm{CEM}$ \\
Fiducial & Fid $=1 \mathrm{or} 2$ & Fid $=1$ or 2 \\
$\mathrm{E}_{T}$ & $\geq 25 \mathrm{GeV}$ & $\geq 25 \mathrm{GeV}$ \\
Track $Z_{0}$ & $\leq 60 \mathrm{~cm}$ & $\leq 60 \mathrm{~cm}$ \\
Track $p_{T}$ & $\geq 15 \mathrm{GeV} / \mathrm{c}$ & $\geq 15 \mathrm{GeV} / \mathrm{c}$ \\
Had $/ \mathrm{em}$ & - & $\leq 0.055+0.00045 \times E$ \\
Isolation & - & $\leq 1.5+0.02 \times E_{T} \mathrm{GeV}$ \\
$L_{S h r}$ & - & $\leq 0.1$ \\
$E / P_{\left(E_{T}<100 \mathrm{GeV}\right)}$ & - & $\leq 2.5+0.015 \times E_{T} \mathrm{GeV}$ \\
$E / P_{\left(E_{T} \geq 100 \mathrm{GeV}\right)}$ & - & Track $p_{T} \geq 25 \mathrm{GeV} / \mathrm{c}$ \\
CES $\Delta Z$ & - & $\leq 5.0 \mathrm{~cm}$ \\
CES $\Delta X$ & - & $\leq 3.0 \mathrm{~cm}$ \\
Conversion & - & false \\
\hline
\end{tabular}

Table 5.1: The CEM selection cuts for the efficiency samples. All cuts are performed with $E$ in units of $\mathrm{GeV}$.

\begin{tabular}{|c|c|}
\hline Variable & PEM Probe (PPEM) \\
\hline Region & $=\mathrm{PEM}$ \\
$\mathrm{E}_{T}$ & $\geq 25 \mathrm{GeV}$ \\
$\mathrm{PES} \eta$ & $1.2 \leq\left|\eta_{P E S}\right| \leq 3.0$ \\
$\mathrm{Had} / \mathrm{em}$ & $\leq 0.125$ \\
Track $Z_{0}$ & $\leq 60 \mathrm{~cm}$ \\
$N_{\text {hits }}^{\text {Si }}$ & $\geq 3$ \\
\hline
\end{tabular}

Table 5.2: The PEM selection cuts for the efficiency samples.

has passed all the probe plug cuts. For N-1 efficiencies, the formulae for the efficiency of $i^{t h}$ cut become for the CEM

$$
\epsilon_{N-1}^{i}=\frac{2 \times N_{C E M-C E M}}{N_{C E M-C E M}+N_{C E M-N 1}^{i}}
$$

and for the PEM

$$
\epsilon_{N-1}^{i}=\frac{N_{T C E M-P E M}}{N_{T C E M-N 1}^{i}}
$$

where the index $N 1$ indicates that the electron has passed all of the appropriate normal cuts with the exception of the $i^{\text {th }}$ cut which it may or may not have passed.

The number of background events present in each sample is estimated following the method described in [55] and summarised in section 5.1.2. The number of background events in each sample is summarised in table 5.3. Only the total efficiencies are 


\begin{tabular}{|c|c|c|}
\hline & Nr of Events & Nr of Background Events \\
\hline CEMCC-CEMCC & 19196 & $110.8 \pm 26.0$ \\
CEMCC-PCEM & 21693 & $781.9 \pm 42.9$ \\
\hline CEMCP-CEMCP & 18086 & $30.5 \pm 11.0$ \\
CEMCP-PCEM & 21386 & $420.2 \pm 35.5$ \\
\hline TCEM-PEM & 19930 & $62.9 \pm 26.1$ \\
TCEM-PPEM & 22248 & $191.5 \pm 29.2$ \\
\hline
\end{tabular}

Table 5.3: Number of events observed in each efficiency category together with the estimated background. The uncertainties are statistical only. Note that the CEMCP-CEMCP and CEMCP-PCEM samples are subsets of the CEMCC-CEMCC and CEMCC-PCEM samples respectively.

\begin{tabular}{|c|c|c|}
\hline Efficiency & Data & Monte Carlo \\
\hline Had/em & $0.9924 \pm 0.0004$ & $0.9892 \pm 0.0002$ \\
Isolation & $0.976 \pm 0.001$ & $0.9768 \pm 0.0003$ \\
$L_{S h r}$ & $0.9904 \pm 0.0005$ & $0.9895 \pm 0.0002$ \\
$\mathrm{E} / \mathrm{P}$ & $0.9995 \pm 0.0001$ & $0.9994 \pm 0.0001$ \\
$\mathrm{CES} \Delta Z$ & $0.9986 \pm 0.0002$ & $0.9993 \pm 0.0001$ \\
$\mathrm{CES} \Delta X$ & $0.9927 \pm 0.0004$ & $0.9933 \pm 0.0002$ \\
Conversion & $0.971 \pm 0.001$ & $0.9720 \pm 0.0003$ \\
\hline
\end{tabular}

Table 5.4: CEM N-1 identification efficiencies at the $Z^{0}$ Pole. The uncertainties are statistical only.

corrected for the presence of background as the N-1 efficiencies are estimated for informational purposes only. The resulting efficiency measurements using data and Monte Carlo generated events are displayed in tables 5.4, 5.5 and 5.6 for the central N-1, plug $\mathrm{N}-1$ and total identification efficiencies respectively. The scale factors in table 5.6 are used to correct the Monte Carlo simulation's estimate of the identification efficiency and are applied multiplicatively. The CEM scale factor is close to one, indicating good agreement between the Monte Carlo simulation and the data. The PEM scale factor is 0.966, which indicates that the modelling of the plug electron identification variables in the Monte Carlo simulation is less well-developed than the modelling of the central electron identification variables. Most of the disagreement comes from the isolation $E_{T}$ and the PEM $\chi_{3 \times 3}^{2}$ efficiencies, perhaps indicating that the modelling of the electron energy deposition in the PEM could be improved in the future.

\subsubsection{Background Estimation}

In the efficiency samples, the largest contribution to the 'fake' electron background are di-jet and $W+$ jet events where the jets have faked the signature of an electron. These 


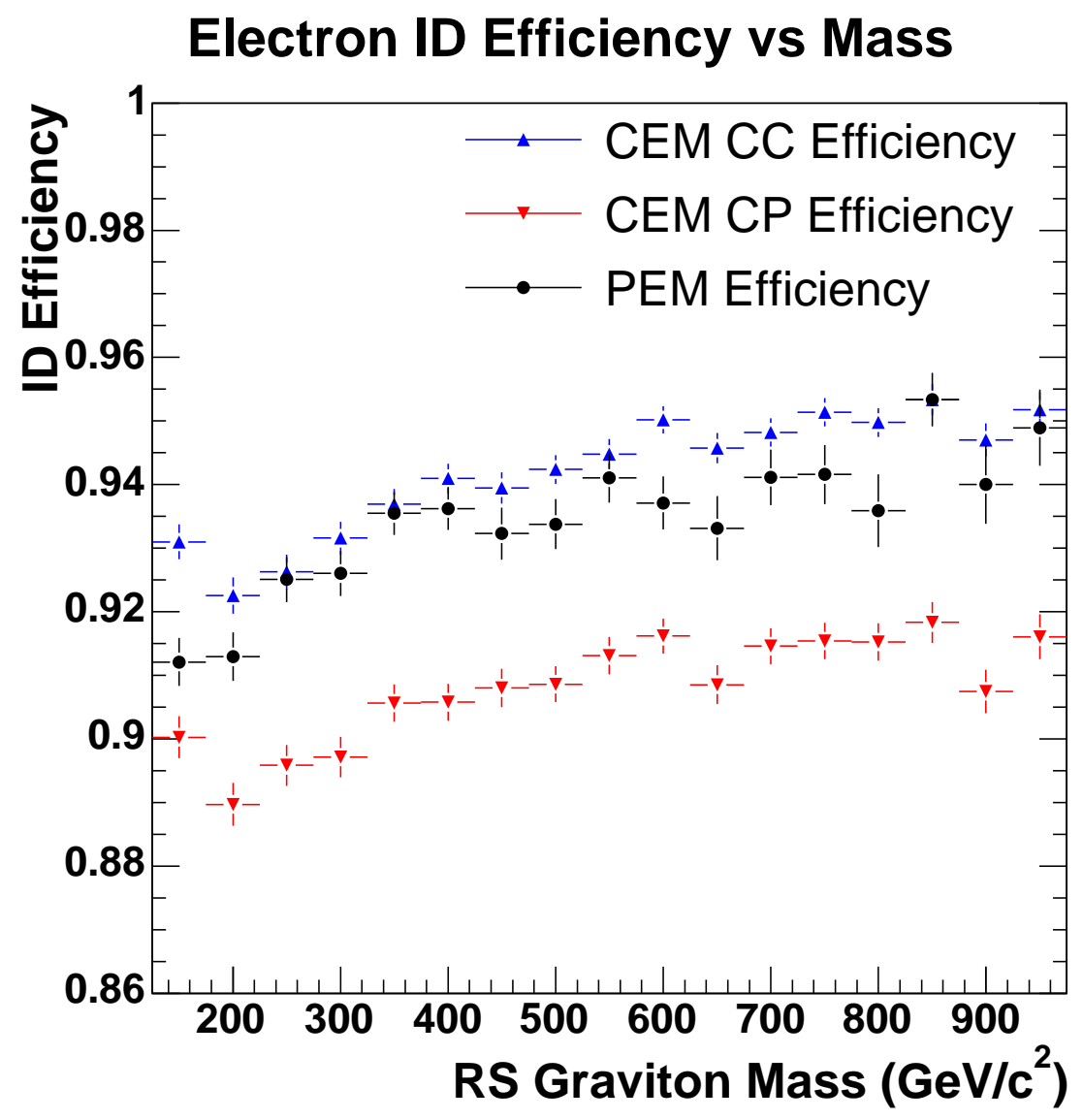

Figure 5.1: Electron Identification efficiencies estimated using the Monte Carlo simulation with the scale factors applied. The cuts are designed to give a gradually increasing efficiency with mass for reasons discussed in appendix A. For the CEM, the efficiency of the $E / p$ cut is distorted by the acceptance requirement of track $p_{T} \geq 15 \mathrm{GeV} / c$. This gives an artificially high efficiency at low mass and is an artifact of separating out the identification efficiency from the acceptance and is removed when the acceptance is included into the total selection efficiency. 


\begin{tabular}{|c|c|c|}
\hline Efficiency & Data & Monte Carlo \\
\hline Had/em & $0.992 \pm 0.001$ & $0.9929 \pm 0.0002$ \\
Isolation & $0.955 \pm 0.001$ & $0.9725 \pm 0.0004$ \\
$\mathrm{PEM} \chi_{3 \times 3}^{2}$ & $0.962 \pm 0.001$ & $0.9735 \pm 0.0004$ \\
\hline
\end{tabular}

Table 5.5: PEM N-1 identification efficiencies at the $Z^{0}$ Pole. The uncertainties are statistical only.

\begin{tabular}{|c|c|c|c|}
\hline Efficiency & Data & Monte Carlo & Scale Factor \\
\hline CEM CC & $0.954 \pm 0.001$ & $0.9461 \pm 0.0004$ & $1.008 \pm 0.001$ \\
CEM CP & $0.926 \pm 0.002$ & $0.920 \pm 0.001$ & $1.007 \pm 0.002$ \\
PEM & $0.901 \pm 0.002$ & $0.933 \pm 0.001$ & $0.966 \pm 0.002$ \\
\hline
\end{tabular}

Table 5.6: Total efficiencies and scale factors at the $Z^{0}$ Pole. The uncertainties are statistical only.

backgrounds are collectively referred to as the 'jet background'. For central electron identification efficiencies, the background in the sample is estimated using the number of same sign events that pass the selection requirements for the sample. Naively the number of same sign events could be taken as the number of background events in the opposite sign sample, assuming that the reconstructed charge of a misidentified jet is uncorrelated with the other object identified as an electron in the event. However there are two corrections to this. The first is a correction for so-called 'trident events', where the electron radiates a hard photon which then converts into an $e^{+} e^{-}$pair. This can lead to the highest $p_{T}$ track associated with the electron being of the opposite sign to the original electron. This effect is estimated from the number of same sign events predicted by the $Z^{0} / \gamma^{*} \rightarrow e^{+} e^{-}$Monte Carlo simulation. The second correction accounts for the fact that a charge correlation is observed in $W+$ jet dominated jet background events [55]. The effect of this correlation is that the number of same sign background events is $40 \%$, rather than $50 \%$, of the total number of background events [5.5. Therefore the number of background events in the opposite sign sample is $\frac{0.6}{0.4}$ times the number of same sign events. Applying both corrections, the total number of background events in the central efficiency samples $\left(N_{j e t}\right)$ is

$$
N_{\text {jet }}=\frac{0.6}{0.4} \times\left(N_{\text {same sign }}^{\text {data }}-N_{\text {samesign }}^{M C}\right)
$$

where $N_{\text {same sign }}^{\text {data }}$ is the number of same sign events in data and $N_{\text {samesign }}^{M C}$ is the number of same sign events predicted by the Monte Carlo simulation. The number of background events in each of the central-central efficiency samples can be found in table 5.3 .

To estimate the number of background events in the samples used to measure the plug efficiency, the same sign method can not be used since the charge of the plug 
electron is not reliably known. Instead, a method using the side bands of the $Z^{0}$ peak is used [55]. The Monte Carlo generated events are first normalised to the data in the mass region of $81<M_{e e}<101 \mathrm{GeV} / c^{2}$. Then the difference between the number of data and Monte Carlo generated events in the mass window of $70<M_{e e}<120 \mathrm{GeV} / c^{2}$ is found. Dividing this number by the mass range of $30 \mathrm{GeV} / c^{2}$ gives the average number of background events per $\mathrm{GeV} / c^{2}$. This average is then multiplied by $20 \mathrm{GeV} / c^{2}$ to obtain an estimate of the number of background events in the $81<M_{e e}<101 \mathrm{GeV} / c^{2}$ efficiency mass window. The number of background events estimated to be present in each of the plug efficiency samples can be found in table 5.3 .

\subsubsection{Systematic Uncertainties on the Identification Efficiency}

There are two sources which contribute to the systematic uncertainty on the electron identification efficiency estimates. First there is an uncertainty on the efficiency scale factor resulting from the uncertainty in estimating the background in the efficiency samples. The standard method used at CDF to evaluate the effect of this uncertainty on the scale factor is to vary the mass window in which the efficiency calculation is performed. The largest resulting deviation in the scale factor from the nominal value is then taken as a systematic uncertainty. The motivation for this method is that the percentage of background events present in the efficiency sample is sensitive to the mass window chosen. If the background estimation is incorrect, the differing mass windows will give different results for the scale factor. The various mass windows chosen, together with the measured scale factor, are shown in tables 5.7 and 5.8 for the CEM and PEM respectively. The scale factors for the various mass windows are close to each other, giving confidence in the background estimation method and the systematic uncertainty on the scale factor is taken as $0.2 \%$ and $0.3 \%$ for the CEM and PEM scale factors respectively.

The second source of systematic uncertainty stems from the fact that the behaviour of very high energy electrons is not known reliably, as no sample with sufficient purity exists at high energy. Thus a $2 \%$ uncertainty is applied to the efficiency estimates at high mass. The value of $2 \%$ is chosen as all the Monte Carlo estimates are within $2 \%$ of a central value. This uncertainty only contributes to the uncertainty on the signal crosssection which is dominated by the $6 \%$ luminosity uncertainty and the uncertainties on the acceptance discused in section 5.2 .

\subsection{Kinematic and Geometrical Acceptance}

The kinematic and geometrical acceptance is estimated using Monte Carlo generated events. The acceptance $(A)$ is calculated from the formula

$$
A=\frac{N^{C A L}}{N^{G E N}}
$$




\begin{tabular}{|c|c|c|c|}
\hline Mass Window $\left(\mathrm{GeV} / c^{2}\right)$ & Data & Monte Carlo & Eff. Scale Factor \\
\hline $71-111$ & $0.954 \pm 0.002$ & $0.9444 \pm 0.0004$ & $1.010 \pm 0.002$ \\
$\mathbf{7 6 - 1 0 6}$ & $\mathbf{0 . 9 5 4} \pm \mathbf{0 . 0 0 1}$ & $\mathbf{0 . 9 4 6 1} \pm \mathbf{0 . 0 0 0 4}$ & $\mathbf{1 . 0 0 8} \pm \mathbf{0 . 0 0 1}$ \\
$81-101$ & $0.956 \pm 0.002$ & $0.9489 \pm 0.0004$ & $1.007 \pm 0.001$ \\
$86-96$ & $0.961 \pm 0.002$ & $0.9541 \pm 0.0004$ & $1.007 \pm 0.001$ \\
\hline
\end{tabular}

Table 5.7: CEM electron identification efficiencies and scale factors for various mass windows, measured using data and Monte Carlo generated events. The uncertainties are statistical only. The largest difference from the nominal value (shown in bold) is taken as a systematic uncertainty.

\begin{tabular}{|c|c|c|c|}
\hline Mass Window $\left(\mathrm{GeV} / c^{2}\right)$ & Data & Monte Carlo & Eff. Scale Factor \\
\hline $71-111$ & $0.898 \pm 0.002$ & $0.932 \pm 0.001$ & $0.963 \pm 0.002$ \\
$76-106$ & $0.899 \pm 0.002$ & $0.933 \pm 0.001$ & $0.964 \pm 0.002$ \\
$\mathbf{8 1 - 1 0 1}$ & $\mathbf{0 . 9 0 1} \pm \mathbf{0 . 0 0 2}$ & $\mathbf{0 . 9 3 3} \pm \mathbf{0 . 0 0 1}$ & $\mathbf{0 . 9 6 6} \pm \mathbf{0 . 0 0 2}$ \\
$86-96$ & $0.907 \pm 0.002$ & $0.937 \pm 0.001$ & $0.968 \pm 0.002$ \\
\hline
\end{tabular}

Table 5.8: PEM electron identification efficiencies and scale factors for various mass windows measured using data and Monte Carlo generated events. The uncertainties are statistical only. The largest difference from the nominal value (shown in bold) is taken as a systematic uncertainty. 
where $N^{G E N}$ is the number of events at the generator level and $N^{C A L}$ is the number of events at the calorimeter level. Events at the calorimeter level are required to have two electrons passing the probe cuts of tables 5.1 and 5.2 appropriate to the region of the electron but with no Phoenix tracking requirement for plug electrons. Events at the generator level are required to be generated at a $z$ position of $|z| \leq 60 \mathrm{~cm}$ to separate out the $z$-vertex cut efficiency. For the $Z^{0}$ acceptance, used later on to calculate the $Z^{0}$ cross-section as a cross-check, a mass window cut of $66<M_{Z}<116 \mathrm{GeV} / c^{2}$ is applied at the generator and calorimeter levels. The acceptance as a function of mass is shown in figure 5.2 for both spin-1 and spin-2 bosons. The total selection efficiency as a function of mass is shown in figure 5.3 for both spin-1 and spin-2 bosons.

The acceptance estimate from the Monte Carlo simulation depends partially on the parton distribution function $(\mathrm{PDF})$ used for the proton and anti-proton. All Monte Carlo generated samples used to estimate an acceptance were generated using CTEQ5L for the proton and anti-proton PDFs. The CTEQ5L PDFs are obtained using a LO QCD fit to a large number of experimental data sets, and therefore there are many sources of uncertainties with non-trivial correlations. The CTEQ collaboration reduces the different sources of uncertainty in the fit into 20 orthogonal eigenvectors. Each of these eigenvectors is fluctuated up and down by $1 \sigma$ to produce a total of 40 new PDF sets. As these PDF sets are not available for the CTEQ5L PDFs, the CTEQ6M PDFs are used for the purposes of estimating the acceptance uncertainty. The relative acceptance uncertainty using the CTEQ6M PDFs taken as the relative uncertainty for the CTEQ5L PDFs. The difference between the two PDFs is small with the acceptance estimate for the central-central and central-plug channels at the $Z^{0}$ pole using the CTEQ5L PDFs being 0.109 and 0.189 respectively, while the corresponding acceptance estimates using CTEQ6M PDFs are 0.111 and 0.190 .

For each individual eigenvector of CTEQ6M PDF set, the Monte Carlo is reweighted using the PDF corresponding to the eigenvector fluctuated up by $1 \sigma$ and then with the PDF corresponding to the eigenvector fluctuated down by $1 \sigma$. For each re-weighting, the acceptance is re-calculated and the difference from the nominal value of the acceptance is taken as the uncertainty on the acceptance resulting from the uncertainty on quantity the eigenvector represents. While each eigenvector is treated as a separate independent uncertainty when setting limits in section 7.2 , sometimes it is convenient to express a single overall uncertainty on the acceptance resulting from all the uncertainties effecting the PDF. The overall uncertainty on the acceptance is obtained by adding in quadrature all the negative uncertainties and adding in quadrature all the positive uncertainties on the acceptance uncertainty resulting from the PDF uncertainties. In the case of a particular eigenvector's $\pm 1 \sigma$ variation both giving an acceptance shift in the same direction, the average is taken and then added in quadrature to the positive or negative sum of the uncertainties as appropriate.

The PDF uncertainties also lead to an uncertainty in the Drell-Yan shape at high masses. For each PDF eigenvector, the Monte Carlo simulated Drell-Yan mass spectrum was re-weighted with the PDFs corresponding to the eigenvector fluctuated up and down by $1 \sigma$. Again, while each eigenvector is treated as an independent source 

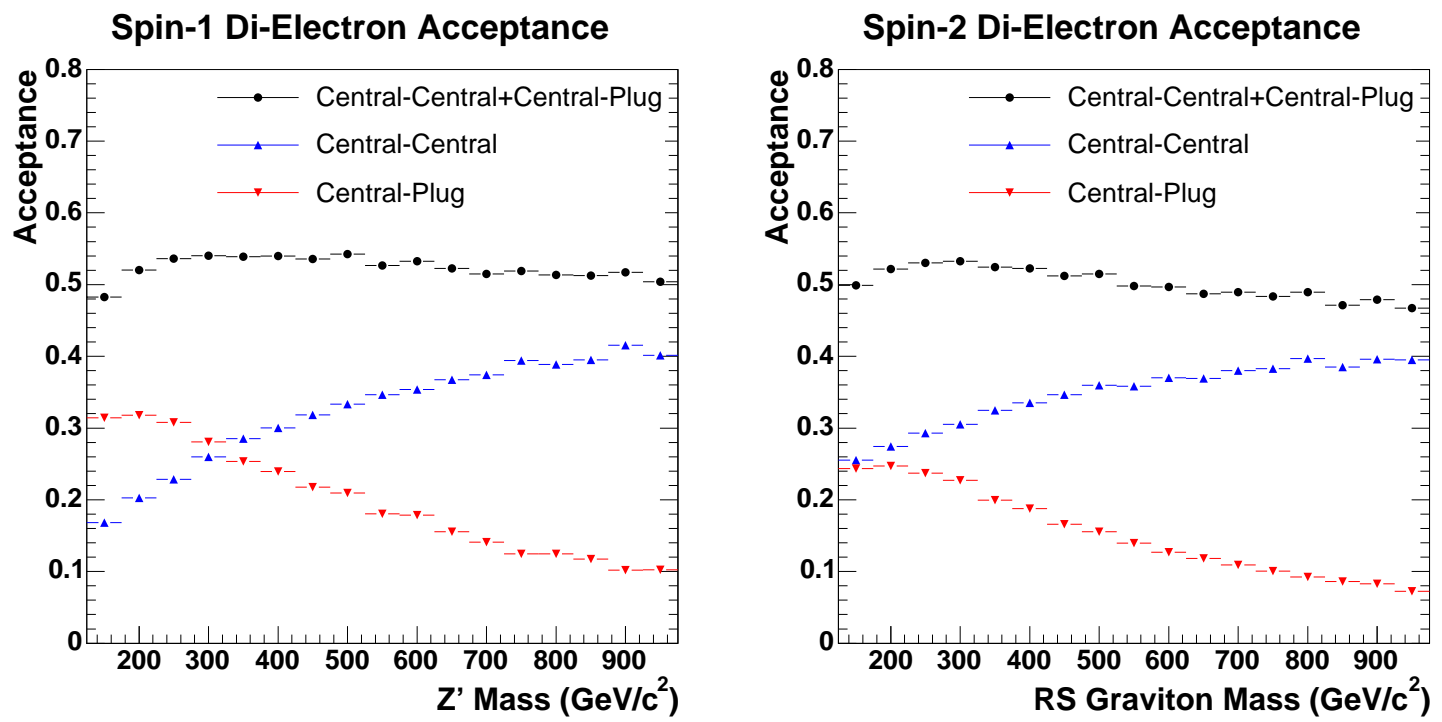

Figure 5.2: Monte Carlo estimated acceptances for neutral spin-1 and spin-2 particles decaying to two electrons as a function of the particle's mass.

of uncertainty for the purposes of setting limits, to obtain the total uncertainty on the Drell-Yan background shape the individual differences in the predicted Drell-Yan shape are summed in an identical manner to the acceptance differences.

Initial state radiation (ISR) also contributes to an uncertainty on the acceptance at high mass. This uncertainty has been evaluated by the high mass di-photon analysis which also looks for a massive narrow resonance decay [45]. The parameters in the Monte Carlo simulation related to the ISR calculation were changed to be half and then double the default values and the resulting change on the acceptance was recorded. This study resulted in a $4 \%$ uncertainity being applied to the acceptance at high mass due to the ISR uncertainity.

\subsection{Acceptance and Efficiency Estimate Cross-Check}

In order to give confidence in the efficiency and acceptance estimates, the $Z^{0} \rightarrow e^{+} e^{-}$ cross-section is measured. Following CDF convention, the cross-section is measured in a mass window of $66 \leq M_{Z} \leq 116 \mathrm{GeV} / c^{2}$ and includes the $\gamma^{*}$ contribution. The cross-section is calculated using the formula

$$
\sigma=\frac{N_{Z}^{\text {data }}-N_{Z}^{b k g}}{A_{z} \epsilon_{I D} \epsilon_{z_{0}} \mathcal{L}}
$$

where $N_{Z}^{\text {data }}$ and $N_{Z}^{b k g}$ are respectively the number of data and background events in the mass window, $A_{Z}$ is the acceptance at the $Z^{0}$ pole, $\mathcal{L}$ is the luminosity of the 

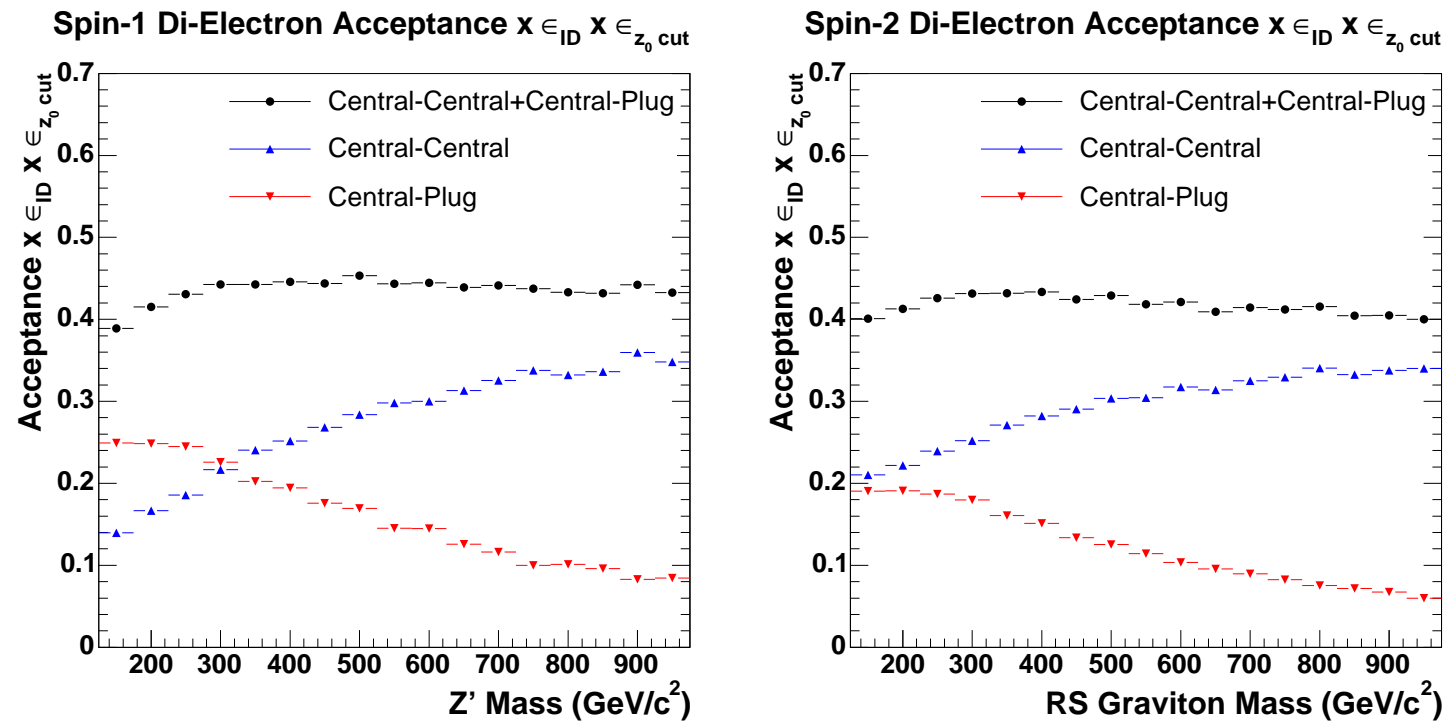

Figure 5.3: Monte Carlo estimated acceptance times efficiency for neutral spin-1 and spin-2 particles decaying to two electrons as a function of the particle's mass.

data sample and $\epsilon_{I D}$ and $\epsilon_{z_{0}}$ are respectively the identification and z-vertex efficiencies. Table 5.9 shows the measured values of these parameters and the resulting cross-section. The measured cross-sections are consistent between the two channels and are consistent with the NLO prediction of $251.3 \pm 5.0 \mathrm{pb}$ [9]. The number of background events in table 5.9 is calculated using the methods described in chapter 6. At the $Z^{0}$ pole, the fraction of background events to real $Z^{0} / \gamma^{*}$ events is small and so while they do affect the cross-section calculation, the calculation is mainly sensitive to the efficiency, acceptance and luminosity estimates. 


\begin{tabular}{|c|c|c|}
\hline & Central-Central & Central-Plug \\
\hline Acceptance $\left(A_{Z}\right)$ & $0.109 \pm 0.002_{\text {sys. }}$ & $0.189 \pm 0.001_{\text {sys. }}$ \\
ID Eff. $\left(\epsilon_{I D}\right)$ & $0.910 \pm 0.002_{\text {stat. }}$ & $0.834 \pm 0.003_{\text {stat. }}$ \\
Z Vertex Eff. $\left(\epsilon_{z_{0}}\right)$ & $0.951 \pm 0.003_{\text {sys. }}$ & $0.951 \pm 0.003_{\text {sys. }}$ \\
Expected Bkg. $\left(N_{Z}^{b k g}\right)$ & $61.5 \pm 23$. sys. $^{\text {byg }}$ & $293.2 \pm 54.2_{\text {sys. }}$ \\
Luminosity $(\mathcal{L})$ & $819 \pm 49.2 \mathrm{pb}^{-1}$ & $819 \pm 49.2 \mathrm{pb}^{-1}$ \\
Observed Events $\left(N_{Z}^{\text {data }}\right)$ & 20303 & 32046 \\
\hline \hline$\sigma . \operatorname{Br}\left(Z^{0} / \rightarrow e^{+} e^{-}\right)(\mathrm{pb})$ & $262.0 \pm 1.8 \pm 1.1 \pm 15.7$ & $258.6 \pm 1.4 \pm 1.9 \pm 15.5$ \\
\hline
\end{tabular}

Table 5.9: The measured $Z^{0} / \gamma^{*} \rightarrow e^{+} e^{-}$cross-section in the range of $66-116 \mathrm{GeV} / c^{2}$ for $819 \mathrm{pb}^{-1}$ of data in the central-central and central-plug channels. The first uncertainty is statistical, the second is systematic and the third is from the $6 \%$ luminosity uncertainty. Only the uncertainties on the acceptance, efficiency and background estimates are included as systematic uncertainties on the cross-section measurement, with the other sources being ignored. 


\section{Chapter 6}

\section{Standard Model Backgrounds and Cross-Checks}

There are four types of backgrounds for a new physics signal in the di-electron channel. These are listed below in order of significance.

1. $Z^{0} / \gamma^{*} \rightarrow e^{+} e^{-}$

The Standard Model Drell-Yan process via s-channel $Z^{0} / \gamma^{*}$, with a final state of two electrons.

2. Jet Background

Multi-jet events where two jets are misidentified as electrons or $W+$ jet events where the $W$ decays to an electron and the jet is misidentified as an electron.

3. Di-Photon

Events where two photons are misidentified as electrons.

4. Electroweak Processes

Hard processes producing the final states:

$W+\gamma \rightarrow e \nu_{e}+\gamma$ where the photon is misidentified as an electron

$W^{+} W^{-} \rightarrow e^{+} e^{-} \nu_{e} \bar{\nu}_{e}$

$W^{ \pm} Z$, where $Z \rightarrow e^{+} e^{-}$

$Z / \gamma^{*} \rightarrow \tau^{+} \tau^{-} \rightarrow e^{+} e^{-} \nu_{\tau} \nu_{e} \overline{\nu_{\tau}} \overline{\nu_{e}}$

$t \bar{t} \rightarrow e^{+} e^{-} \nu_{e} \overline{\nu_{e}} b \bar{b}$.

\section{1 $Z^{0} / \gamma^{*} \rightarrow e^{+} e^{-}$Background}

The SM Drell-Yan process represents an irreducible background to a new physics process producing two electrons. This background is estimated using Monte Carlo generated events normalised to the data at the $Z^{0}$ pole in a window of $76-106 \mathrm{GeV} / c^{2}$ for central-central events and in a window of $81-101 \mathrm{GeV} / c^{2}$ for central-plug events. To 


\begin{tabular}{|c|c|}
\hline region & Estimated Background \\
\hline central-central & $33.9 \pm 26.8_{\text {stat. }} \pm 11.3_{\text {sys. }}$ \\
central-plug & $463.8 \pm 68.0_{\text {stat. }} \pm 124.1_{\text {sys. }}$ \\
\hline
\end{tabular}

Table 6.1: Number of jet background events estimated in data

improve the statistics at high mass, a series of samples were generated at minimum $Z^{0} / \gamma^{*}$ masses of $110,150,200,300,500$ and $800 \mathrm{GeV} / c^{2}$, with each sample consisting of 10,000 events. The resulting mass spectrum predictions, after the samples are appropriately weighted, are shown in figure 6.1. The uncertainty on the normalisation is taken to be the difference between the normalisations of the central-central and central-plug channels and is found to be $3.8 \%$.

\subsection{Jet Background}

The jet background is a reducible background resulting from jets faking the signature of an electron and so passing the electron identification cuts. The number of jet background events is estimated using the isolation vs isolation method described in section 6.2.1. The number of jet background events estimated by both methods can be found in table 6.1. The shape of the background is estimated using an electron-like jet sample, where one jet is required to pass the electron identification cuts. This is explained in more detail in section 6.2.2,

\subsubsection{Isolation vs Isolation Normalisation Method}

In this method of estimating the number of jet background events, the isolation $E_{T}^{c o r r}$ of the two electrons are plotted against each other and the resulting plot is divided into four regions labelled A, B, C and D, as shown in figure 6.2, Region A is the signal dominated region and the other three regions are all background dominated. While for central-plug events, there is a clear difference between the two electrons, there is no such distinction between the electrons in central-central events. Therefore in centralcentral events, the axis an electron is plotted along, and thus whether it is labelled as 1 or 2 , is determined randomly.

The boundaries of each region, together with the number of events observed in the region, are given in tables 6.2 and 6.3 for the central-central and central-plug channels respectively. Assuming that the isolation of the two electrons are uncorrelated, the ratio of the number of events in region $\mathrm{A}$ to region $\mathrm{B}$ is equal to the ratio of the number of events in region $\mathrm{C}$ to region $\mathrm{D}$. Therefore the number of jet background events $\left(N_{\text {jet }}\right)$ in the signal region A can be estimated using the following formula

$$
N_{\text {jet }}=N_{B} \times N_{C} / N_{D}
$$

where $N_{B}, N_{C}$ and $N_{D}$ are the number of events in regions B, C and D respectively. 
$\mathbf{Z}^{0} / \gamma^{*} \rightarrow \mathbf{e}^{+} \mathbf{e}^{-}$Background Prediction

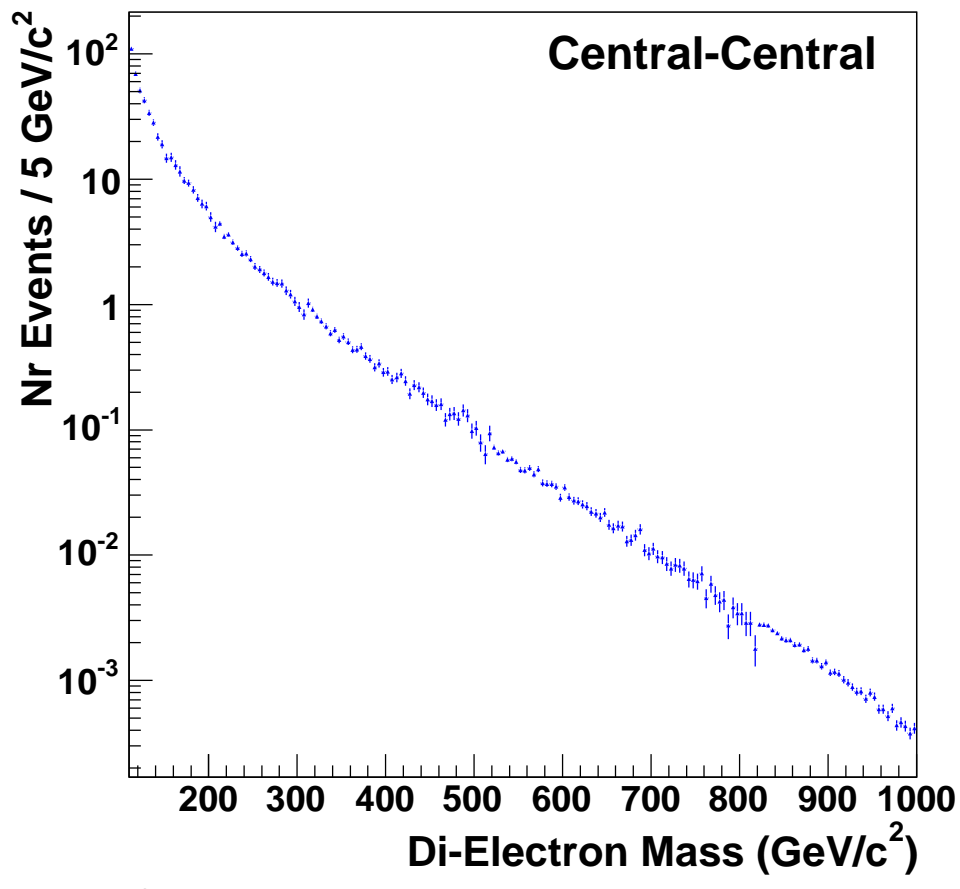

$\mathrm{Z}^{0} / \gamma^{*} \rightarrow \mathrm{e}^{+} \mathrm{e}^{-}$Background Prediction

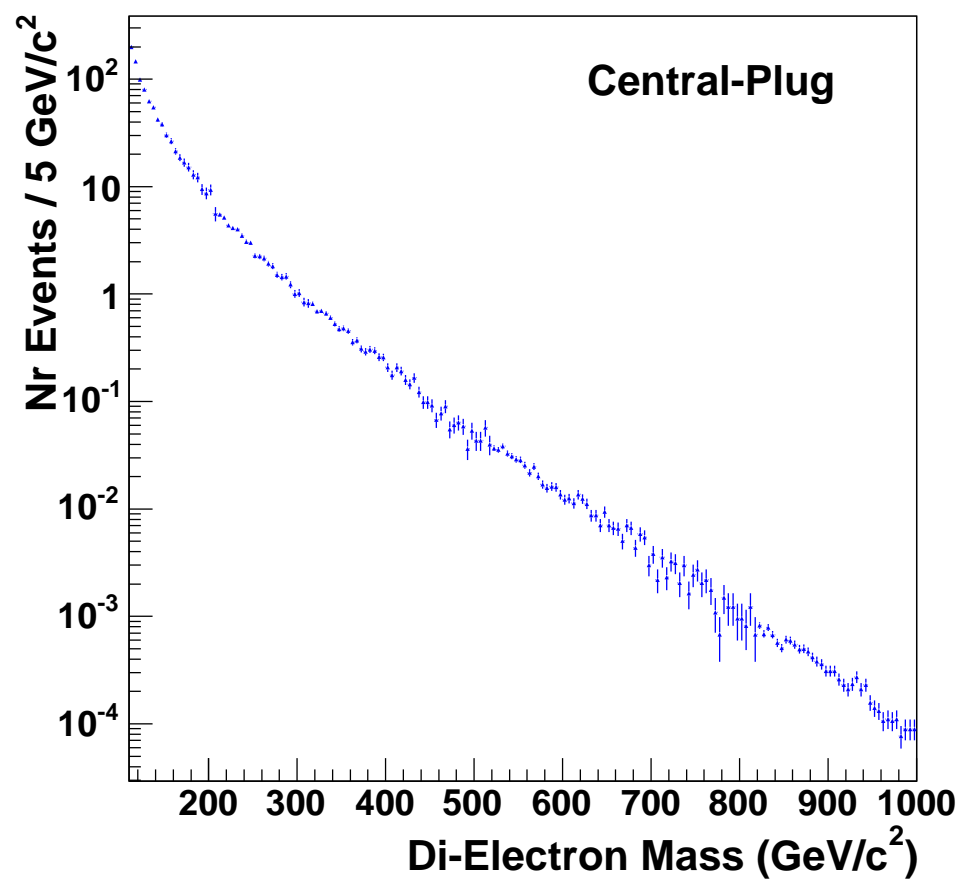

Figure 6.1: The predicted Drell-Yan mass spectra for the central-central and centralplug channels. 

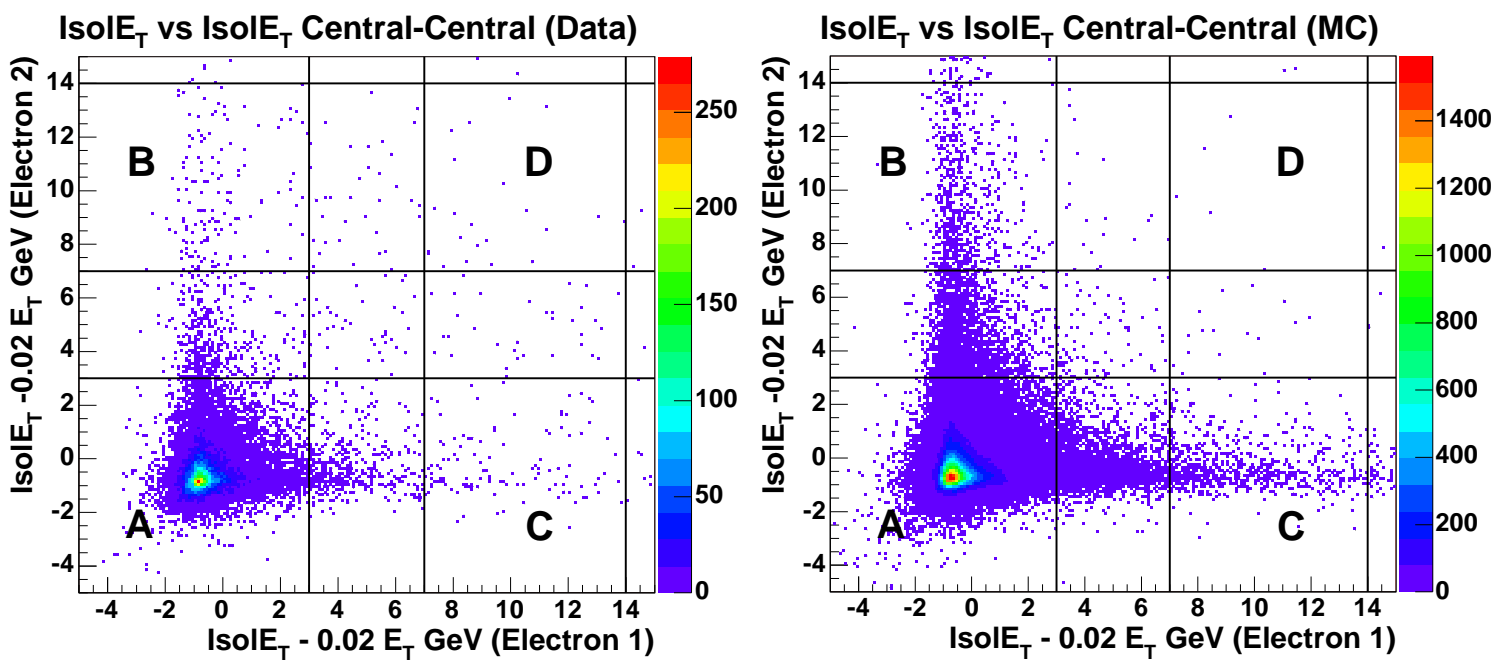

IsolE $_{\mathrm{T}}$ vs IsolE $\mathrm{T}_{\mathrm{T}}$ Central-Plug (Data)

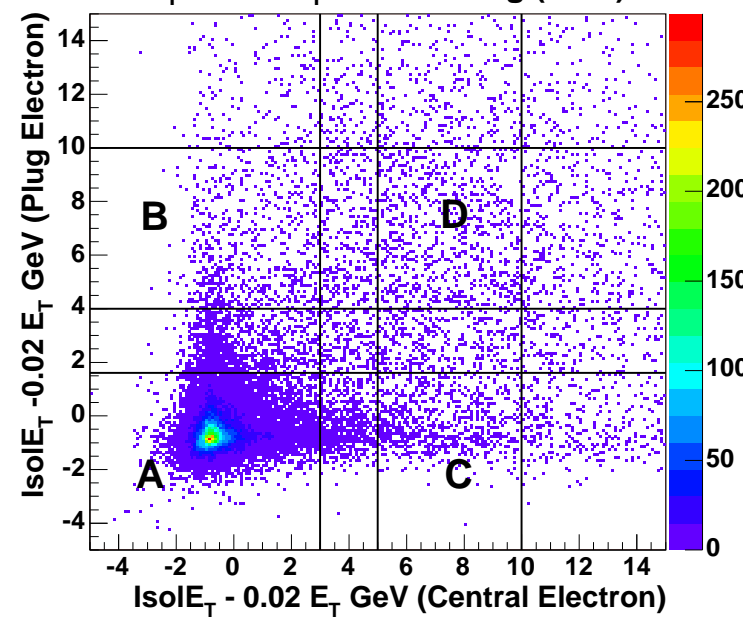

IsolE $_{\mathrm{T}}$ vs IsolE $\mathrm{T}_{\mathrm{T}}$ Central-Plug (MC)

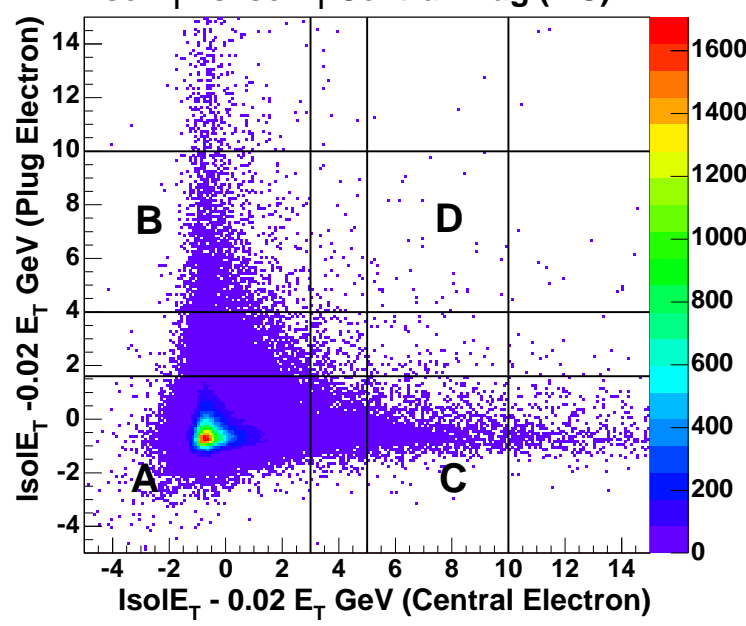

Figure 6.2: Electron isolation $E_{T}-0.02 E_{T}$ vs electron isolation $E_{T}-0.02 E_{T}$ for data and $Z^{0} / \gamma^{*} \rightarrow e^{+} e^{-}$Monte Carlo simulated events in central-central and central-plug regions. Region $\mathrm{A}$ is the signal region while regions $\mathrm{B}, \mathrm{C}$ and $\mathrm{D}$ are the background dominated regions.

\begin{tabular}{|c|c|c|}
\hline & Isol $E_{T}^{\text {corr }}<3.0 \mathrm{GeV}$ & $7.0<$ Isol $E_{T}^{\text {corr }}<14.0 \mathrm{GeV}$ \\
\hline Isol $E_{T}^{\text {corr }}<3.0 \mathrm{GeV}$ & $21084(\mathrm{~A})$ & $121(\mathrm{~B})$ \\
$7.0<\mathrm{Isol} E_{T}^{\text {corr }}<14.0 \mathrm{GeV}$ & $145(\mathrm{C})$ & $22(\mathrm{D})$ \\
\hline
\end{tabular}

Table 6.2: Number of central-central events observed in the data in the four isolation $E_{T}^{c o r r}$ regions $\mathrm{A}, \mathrm{B}, \mathrm{C}$ and $\mathrm{D}$. 


\begin{tabular}{|c|c|c|}
\hline & Isol $E_{T}^{\text {corr }}<1.6 \mathrm{GeV}$ & $4.0<$ Isol $E_{T}^{\text {corr }}<10.0 \mathrm{GeV}$ \\
\hline Isol $E_{T}^{\text {corr }}<3.0 \mathrm{GeV}$ & $33231(\mathrm{~A})$ & $359(\mathrm{~B})$ \\
$5.0<$ Isol $E_{T}^{\text {corr }}<10.0 \mathrm{GeV}$ & $562(\mathrm{C})$ & $152(\mathrm{D})$ \\
\hline
\end{tabular}

Table 6.3: Number of central-plug events observed in data in the four isolation $E_{T}^{\text {corr }}$ regions $\mathrm{A}, \mathrm{B}, \mathrm{C}$ and $\mathrm{D}$.

\begin{tabular}{|c|c|c|}
\hline & Isol $E_{T}^{\text {corr }}<3.0 \mathrm{GeV}$ & $7.0<$ Isol $E_{T}^{\text {corr }}<14.0 \mathrm{GeV}$ \\
\hline Isol $E_{T}^{\text {corr }}<3.0 \mathrm{GeV}$ & - & $104.4 \pm 3.8(\mathrm{~B})$ \\
$7.0<$ Isol $E_{T}^{\text {corr }}<14.0 \mathrm{GeV}$ & $101.4 \pm 3.8(\mathrm{C})$ & $0.7 \pm 0.3(\mathrm{D})$ \\
\hline
\end{tabular}

Table 6.4: Number of Drell-Yan central-central events in the three background dominated isolation $E_{T}^{c o r r}$ regions B, C and D predicted by Monte Carlo simulation. Region $\mathrm{A}$ is not shown as it is the signal region. The errors are statistical only.

\begin{tabular}{|c|c|c|}
\hline & Isol $E_{T}^{\text {corr }}<3.0 \mathrm{GeV}$ & $7.0<$ Isol $E_{T}^{\text {corr }}<14.0 \mathrm{GeV}$ \\
\hline Isol $E_{T}^{\text {corr }}<3.0 \mathrm{GeV}$ & - & $166.2 \pm 4.8(\mathrm{~B})$ \\
$7.0<\mathrm{Isol} E_{T}^{\text {corr }}<14.0 \mathrm{GeV}$ & $200.1 \pm 5.3(\mathrm{C})$ & $1.6 \pm 0.5(\mathrm{D})$ \\
\hline
\end{tabular}

Table 6.5: Number of Drell-Yan central-plug events in the three background dominated isolation $E_{T}^{c o r r}$ regions $\mathrm{B}, \mathrm{C}$ and D predicted by Monte Carlo simulation. Region A is not shown as it is the signal region. The errors are statistical only. 


\begin{tabular}{|c|c|c|}
\hline Region B Boundaries (ele2) & Region C Boundaries (ele1) & Background Estimate \\
\hline $7.0<$ Isol $E_{\mathbf{T}}^{\text {corr }}<\mathbf{1 4 . 0}$ & $\mathbf{7 . 0}<$ Isol $\mathbf{E}_{\mathbf{T}}^{\text {corr }}<\mathbf{1 4 . 0}$ & $\mathbf{3 3 . 9} \pm \mathbf{2 6 . 8}$ \\
$6.0<$ Isol $E_{T}^{\text {corr }}<14.0$ & $7.0<$ Isol $E_{T}^{\text {corr }}<14.0$ & $43.4 \pm 27.2$ \\
$6.0<$ Isol $E_{T}^{\text {corr }}<13.0$ & $7.0<$ Isol $E_{T}^{\text {corr }}<14.0$ & $45.2 \pm 28.7$ \\
$8.0<$ Isol $E_{T}^{\text {corr }}<10.0$ & $7.0<$ Isol $E_{T}^{\text {corr }}<14.0$ & $35.0 \pm 32.4$ \\
$5.0<$ Isol $E_{T}^{\text {corr }}<13.0$ & $7.0<$ Isol $E_{T}^{\text {corr }}<14.0$ & $36.6 \pm 23.3$ \\
$7.0<$ Isol $E_{T}^{\text {corr }}<14.0$ & $6.0<$ Isol $E_{T}^{\text {corr }}<13.0$ & $31.6 \pm 24.8$ \\
$7.0<$ Isol $E_{T}^{\text {corr }}<14.0$ & $8.0<$ Isol $E_{T}^{\text {corr }}<15.0$ & $41.8 \pm 33.4$ \\
$7.0<$ Isol $E_{T}^{\text {corr }}<14.0$ & $5.0<$ Isol $E_{T}^{\text {corr }}<12.0$ & $22.5 \pm 18.4$ \\
$6.0<$ Isol $E_{T}^{\text {corr }}<12.0$ & $6.0<$ Isol $E_{T}^{\text {corr }}<12.0$ & $30.8 \pm 23.7$ \\
$5.0<$ Isol $E_{T}^{\text {corr }}<11.0$ & $9.0<$ Isol $E_{T}^{\text {corr }}<14.0$ & $25.7 \pm 23.6$ \\
$9.0<$ Isol $E_{T}^{\text {corr }}<15.0$ & $6.0<$ Isol $E_{T}^{\text {corr }}<10.0$ & $34.2 \pm 29.7$ \\
\hline
\end{tabular}

Table 6.6: The jet background estimate for central-central events using the isol $E_{T}^{\text {corr }}$ vs isol $E_{T}^{c o r r}$ method for a variety of choices of the background dominated region boundaries. The nominal choice of region boundaries and the resulting estimate of the jet background is shown in bold. The errors are statistical only.

The background dominated regions will contain some genuine di-electron events which will distort the background estimation. To correct for this, in each region, the number of Drell-Yan events predicted by the Monte Carlo simulation is subtracted from the number of observed events in that region. The number of Drell-Yan events predicted in each of the background dominated regions are shown in tables 6.4 and 6.5 for the central-central and central-plug channels respectively.

\section{Uncertainties on the Background Estimate}

The effect of the arbitrary choice of the boundaries of the background dominated regions on the jet background estimate is investigated by varying the boundaries and recording the resulting background estimate. A systematic error on the method is taken to be the biggest deviation from the nominal value occurring from sensibly changing the boundaries of the background dominated regions. Tables 6.6 and 6.7 show the jet background estimates for a variety of choices of region boundaries for the background dominated regions for the central-central and central-plug channels respectively. Having the boundaries too close to the signal region produced large deviations due to the imperfect modelling of the signal events in the Monte Carlo simulation, whereas having the boundaries too far away also produced large deviations due to very limited statistics.

A further systematic uncertainty on the method is due to the imperfect modelling of the isolation $E_{T}$ variable in the Monte Carlo simulation. While the isolation $E_{T}$ of an electron in the central region is modelled well, in the plug region the isolation $E_{T}$ of electrons in Monte Carlo generated events appears to be offset from the isolation $E_{T}$ 


\begin{tabular}{|c|c|c|}
\hline Region B Boundaries $(\mathrm{PEM})$ & Region C Boundaries $(\mathrm{CEM})$ & Background Estimate \\
\hline $\mathbf{4 . 0}<$ Isol $\mathbf{E}_{\mathbf{T}}^{\text {corr }}<\mathbf{1 0 . 0}$ & $\mathbf{5 . 0}<$ Isol $\mathbf{E}_{\mathbf{T}}^{\text {corr }}<\mathbf{1 0 . 0}$ & $\mathbf{4 6 3 . 8} \pm \mathbf{6 8 . 0}$ \\
$5.0<$ Isol $E_{T}^{\text {corr }}<11.0$ & $5.0<$ Isol $E_{T}^{\text {corr }}<10.0$ & $476.3 \pm 76.4$ \\
$3.0<$ Isol $E_{T}^{\text {corr }}<9.0$ & $5.0<$ Isol $E_{T}^{\text {corr }}<10.0$ & $587.9 \pm 74.6$ \\
$3.0<$ Isol $E_{T}^{\text {corr }}<9.0$ & $6.0<$ Isol $E_{T}^{\text {corr }}<9.0$ & $535.9 \pm 80.2$ \\
$3.0<$ Isol $E_{T}^{\text {corr }}<9.0$ & $4.0<$ Isol $E_{T}^{\text {corr }}<12.0$ & $537.0 \pm 62.0$ \\
$6.0<$ Isol $E_{T}^{\text {corr }}<10.0$ & $5.0<$ Isol $E_{T}^{\text {corr }}<10.0$ & $494.5 \pm 94.0$ \\
$6.0<$ Isol $E_{T}^{\text {corr }}<12.0$ & $5.0<$ Isol $E_{T}^{\text {corr }}<9.0$ & $534.8 \pm 97.3$ \\
$4.0<$ Isol $E_{T}^{\text {corr }}<10.0$ & $4.0<$ Isol $E_{T}^{\text {corr }}<12.0$ & $434.1 \pm 58.6$ \\
$5.0<$ Isol $E_{T}^{\text {corr }}<10.0$ & $7.0<$ Isol $E_{T}^{\text {corr }}<12.0$ & $385.7 \pm 69.9$ \\
$7.0<$ Isol $E_{T}^{\text {corr }}<12.0$ & $7.0<$ Isol $E_{T}^{\text {corr }}<12.0$ & $425.4 \pm 93.2$ \\
$4.0<$ Isol $E_{T}^{\text {corr }}<12.0$ & $4.0<$ Isol $E_{T}^{\text {corr }}<8.0$ & $532.7 \pm 77.9$ \\
\hline
\end{tabular}

Table 6.7: The jet background estimate for central-plug events using the isol $E_{T}^{\text {corr }}$ vs isol $E_{T}^{c o r r}$ method for a variety of choices of the background dominated region boundaries. The nominal choice of region boundaries and the resulting estimate of the jet background is shown in bold. The errors are statistical only.

\begin{tabular}{|c|c|c|}
\hline \multirow{2}{*}{ source } & \multicolumn{2}{|c|}{ Uncertainty } \\
\cline { 2 - 3 } & Central-Central & Central-Plug \\
\hline statistical & $26.8(80.1 \%)$ & $68.0(14.6 \%)$ \\
region boundary choice & $11.3(42.2 \%)$ & $124.1(26.8 \%)$ \\
MC isolation $E_{T}$ & - & $44.7(9.6 \%)$ \\
\hline
\end{tabular}

Table 6.8: Uncertainties on the number of jet background events estimated using the isolation vs isolation method

of electrons observed in the data by $0.18 \mathrm{GeV}$. This can be seen from the isolation $E_{T}$ distribution shown in figure 6.3. To check the sensitivity of the background estimation method to a discrepancy of this size in the Monte Carlo simulation, $0.18 \mathrm{GeV}$ is added to the isolation $E_{T}$ of plug electrons in Monte Carlo generated events. This gives a $9.6 \%$ difference in resulting background estimate which is small compared to the $26.8 \%$ uncertainty resulting from the arbitrary choice of the background dominated regions. Therefore the isolation $E_{T}$ discrepancy does not significantly effect the background estimation and is simply treated as an additional systematic on the method.

There is also a sizable statistical uncertainty on the jet background estimation due to limited statistics in the isolation regions. The size of the of uncertainty on the jet background estimate arising from each of these sources is summarised in table 6.8. To obtain the total uncertainty on the jet background, these three uncertainties are added in quadrature. 


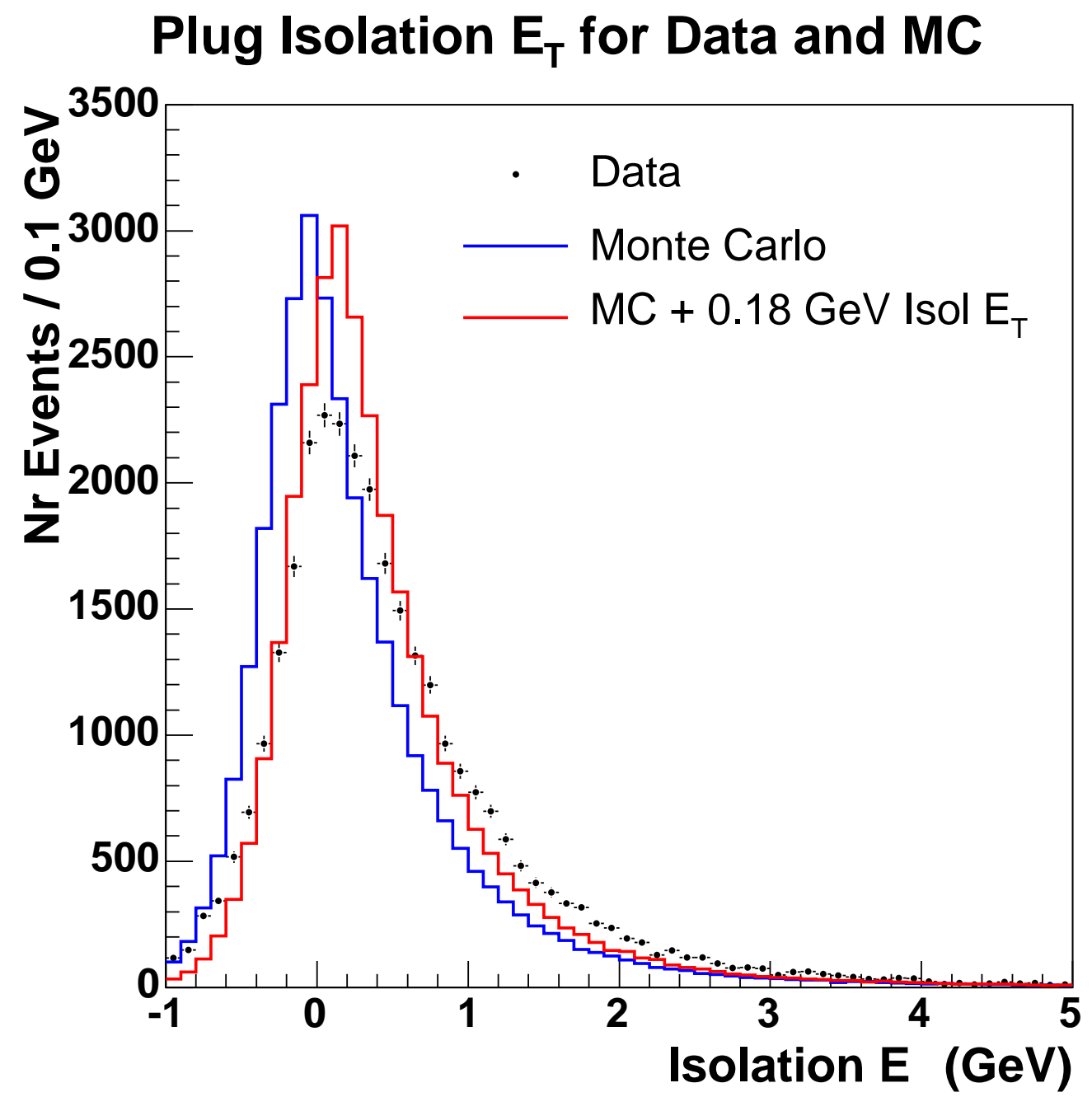

Figure 6.3: The plug electron isolation $E_{T}$ for data events, $Z^{0} / \gamma^{*} \rightarrow e^{+} e^{-}$Monte Carlo generated events and $Z^{0} / \gamma^{*} \rightarrow e^{+} e^{-}$Monte Carlo generated events with 0.18 $\mathrm{GeV}$ added. The data has a sizable jet background which increases with increasing isolation $E_{T}$ and will effect the tails but should not effect the peak position. This accounts for some of the disagreement between the data and the Monte Carlo. 


\begin{tabular}{|l|c|c|}
\hline & Central-Central & Central-Plug \\
\hline Region B & $4(24.1 \%)$ & $14(7.3 \%)$ \\
Region C & $6(13.8 \%)$ & $7(1.9 \%)$ \\
Region D & $2(9.3 \%)$ & $2(1.3 \%)$ \\
\hline
\end{tabular}

Table 6.9: The number of events in each background dominated isolation $E_{T}$ region with $S_{M E T}>2$. The number of events as percentage of the total number of jet events in that region is also shown.

\section{Effect of the Presence of $W+$ jet Events on the Background Estimate}

A possible problem with this method is that it will overestimate the $W+j$ et background. A $W+$ jet background event will have one genuine electron and one jet faking an electron. Therefore $W+$ jet events will be present in regions $\mathrm{A}, \mathrm{B}$ and $\mathrm{C}$ but not $\mathrm{D}$ and this will lead to an overestimation of the background which is approximately the number of $W+$ jet events in region $\mathrm{B}$ times the number of $W+$ jet events in region $\mathrm{C}$ divided by the total number of jet events in region $\mathrm{D}$. If the $W+$ jet background is small compared to the jet background the overestimation is negligible. To estimate the number of $W+$ jet events present in the data sample, the number of events with $E_{\mathrm{T}}$ significance $\left(S_{M E T}\right)>2$ is found. The $\mathbb{E}_{\mathrm{T}}$ significance is defined as

$$
S_{M E T}=\frac{\not_{\mathrm{T}}}{\sqrt{\sum E_{T}^{i}}}
$$

where $E_{\mathrm{T}}$ is the missing $E_{T}$ in the event and $\sum E_{T}^{i}$ is a scalar sum of the EM and hadronic transverse energies over all calorimeter towers. $S_{M E T}$ is standard variable at CDF used to identify the presence of neutrinos. A typical $S_{M E T}$ cut range is from 2 to 2.5 so choosing a cut of $>2$ means that most events with $\nu$ production should be selected although there will still be a large $Z^{0}$ contamination.

The number of events with $S_{M E T}>2$ in each of the isolation regions is shown in table 6.9. From the number of $W+$ jet events estimated to be in regions $\mathrm{B}$ and $\mathrm{D}$, the jet background is overestimated by about $3.3 \%$ and $0.1 \%$ in the central-central and central-plug channels respectively. However, this is an overestimate of the effect as it is conservatively assumed that all events with $S_{M E T}>2$ in region $\mathrm{B}$ and $\mathrm{C}$ are $W+j e t$ events. Given the large statistical and systematic errors associated with this method, the effect of the overestimation of the $W+$ jet contribution on the jet background estimate is negligible.

\subsubsection{Jet Background Shape}

The estimate of the shape of the jet background is obtained from an electron-like jet sample. This sample is constructed by requiring one and only one 'electron 11 passing

\footnotetext{
${ }^{1}$ In this section 'electron' is taken to mean jet which passes the electron selection requirements.
} 


\begin{tabular}{|c|c|c|}
\hline Variable & CEM Loose & PEM Loose \\
\hline Region & $=\mathrm{CEM}$ & $=\mathrm{PEM}$ \\
$\mathrm{E}_{T}$ & $\geq 25 \mathrm{GeV}$ & $\geq 25 \mathrm{GeV}$ \\
$\mathrm{PES} \eta_{2 D}$ & - & $1.2 \leq|\eta| \leq 3.0$ \\
Track $p_{T}$ & $\geq 15 \mathrm{GeV}$ & - \\
$\mathrm{Had} / \mathrm{em}$ & $\leq 0.055+0.00045 \times E$ & $\leq 0.05$ for $E \leq 100 \mathrm{GeV}$ \\
& & $\leq .05+0.026 \ln (E / 100)$ for $E>100 \mathrm{GeV}$ \\
\hline
\end{tabular}

Table 6.10: The loose selection cuts used for selecting an electron-like jet sample. For the had/em cut, the energy is in units of GeV.

the loose selection requirements of table 6.10 and one additional jet with $E_{T}>25 \mathrm{GeV}$ and $|\eta|<1.0$ or $1.2<|\eta|<3.0$, depending whether the jet is in the central or plug regions. The partner jet is also required to fail the had/em cut when reconstructed as a jet. Events are also required to pass the Z_NOTRACK trigger to ensure similarity to normal di-electron sample. The requirements of only a single 'electron' in the event and that the partner jet fails the had/em cut reduces the $Z^{0} \rightarrow e^{+} e^{-}$contamination of the background sample. No $\mathbb{E}_{\mathrm{T}}$ cut is applied in order to allow $W+$ jet events to enter the sample. The loose 'electron' is then required to pass the full selection cuts of tables 4.2 and 4.3 as appropriate. The invariant mass spectrum of the 'electron' and the highest $E_{T}$ jet is then used to obtain the shape of the jet background assuming the mass spectrum shape is not significantly altered when the second jet is required to fake an electron. A measure of the validity of this assumption is taken as a systematic uncertainty on the method and this is described later on in this section.

When a jet is reconstructed as an electron, the jet will have a lower overall energy due to the smaller calorimeter area summed over by the electron reconstruction algorithm as well as the electron reconstruction algorithm not including energy deposited in the hadronic calorimeter in its overall energy. To correct for this, an $E_{T}$ dependent scale factor to correct the jet energy is obtained by taking the ratio between the $E_{T}$ of the jet reconstructed as an electron and the $E_{T}$ of the jet reconstructed as a jet for jets which pass the full set of electron selection cuts. This scale factor, together with its $E_{T}$ dependence, is shown in figure 6.4.

This scale factor is then applied to the jet before plotting the invariant mass spectrum of the 'electron' and jet. Due to limited statistics at high mass, an exponential is fitted to the mass spectrum in the range of $150-350 \mathrm{GeV} / c^{2}$ and then extrapolated to high masses. The mass spectra for the central-central and central-plug regions, together with the fitted exponential functions, are shown in figure 6.5.

Only one of the two jets is required to fake an electron when estimating the jet background shape. The resulting mass spectrum shape is assumed to be the same as true jet background mass spectrum shape where both jets fake electrons. However the probability for the second jet to fake an electron will have an $E_{T}$ dependence and this will cause the two mass spectra to differ to some degree. To estimate the effect of this, 
the probability for a jet which passes the loose selection requirements to pass the full set of selection cuts as a function of $E_{T}$ is calculated. Events are then weighted by this probability using the $E_{T}$ of the non-faking jet as an input. The resulting shape estimates are shown in figure 6.6. with the difference between the weighted di-jet mass spectrum and the unweighted di-jet mass spectrum taken as a systematic uncertainty on the jet background shape.

\subsection{Di-Photon Background}

The di-photon background is a reducible background resulting from one or two photons converting into a $e^{+} e^{-}$pair in the tracking volume. The electrons of the $e^{+} e^{-}$pair will leave tracks in the detector thus allowing the event to pass the central selection cuts. In the case of the plug, there are no tracking requirements and so only the central photon is required to convert, leading to a larger background in the central-plug channel compared to the central-central channel. The size and shape of the background is estimated using Monte Carlo generated di-photon events normalised to the theoretically expected next-to-leading order (NLO) cross section of $185.8 \mathrm{pb}$ [58]. The error on the normalisation is dominated by the conversion and luminosity uncertainties and so no theoretical error is taken on the cross-section.

\subsection{Electroweak Processes Background}

The electroweak background is a reducible background where the two electrons are genuine electrons but are produced in separate W, top, or $\tau$ decays. The background could be reduced by applying a $\mathbb{E}_{\mathrm{T}}$ significance cut since the electrons will be produced in association with neutrinos. However, since this search is an inclusive one, and the electroweak backgrounds are small, a $\mathbb{E}_{\mathrm{T}}$ cut is neither desirable nor necessary. The main processes that contribute are $t \bar{t}, \tau^{+} \tau^{-}, W W, W Z, W+\gamma$. The size and shape of the background is estimated using Monte Carlo generated events for each process, with the Monte Carlo generated events normalised to the theoretically expected NLO cross sections of table 6.11.

\subsection{Non-Drell-Yan Background Cross-Check}

To give further confidence that the background estimates at high mass are correct, two control samples are defined in which any new physics signal is expected to be negligible. Defining these control samples is essential to the search as without them, it would be more difficult to discern if any excess observed in the data is due to incorrect background modelling or new physics. This cross-check was done for the first $368 \mathrm{pb}^{-1}$ of data so that the background estimate could be checked before unblinding the last of the data. Both of these samples have enhanced levels of di-photon and jet background 


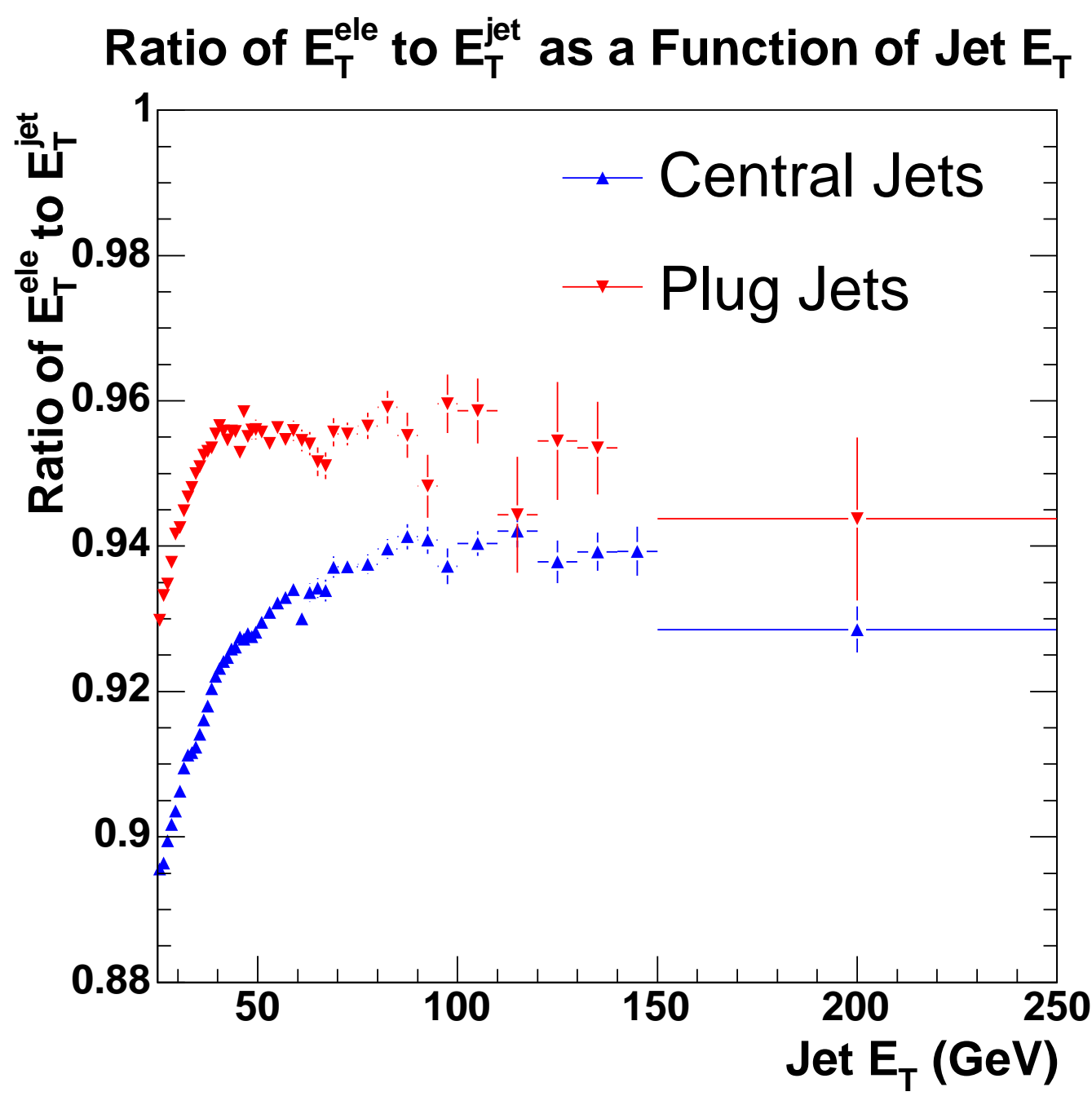

Figure 6.4: The ratio of electron/jet reconstructed $E_{T}$ for a jet. As the $E_{T}$ of the jet increases, the jet becomes more collimated and therefore more of the jet's energy is deposited in the 3-4 towers which make up the electron cluster, making the electron/jet $E_{T}$ ratio increase until almost all the jet's energy is within the towers comprising the electron cluster. The PEM has a larger electron cluster size than the CEM which partially accounts for its higher electron/jet $E_{T}$ ratio. The energy in the hadronic calorimeter is not included in the electron energy and this accounts for the lower electron energy at high $E_{T}$. The $\mathrm{had} / \mathrm{em}$ cut sets a limit on the ratio of the hadronic energy allowed to be present. This cut is lower in the PEM which leads to a higher ratio of electron/jet energy than CEM. As the amount of hadronic energy present is allowed to slowly increase with increasing $E_{T}$, this accounts for the slight decrease in the electron/jet $E_{T}$ ratio at high jet $E_{T}$. 
QCD Background Mass Spectrum
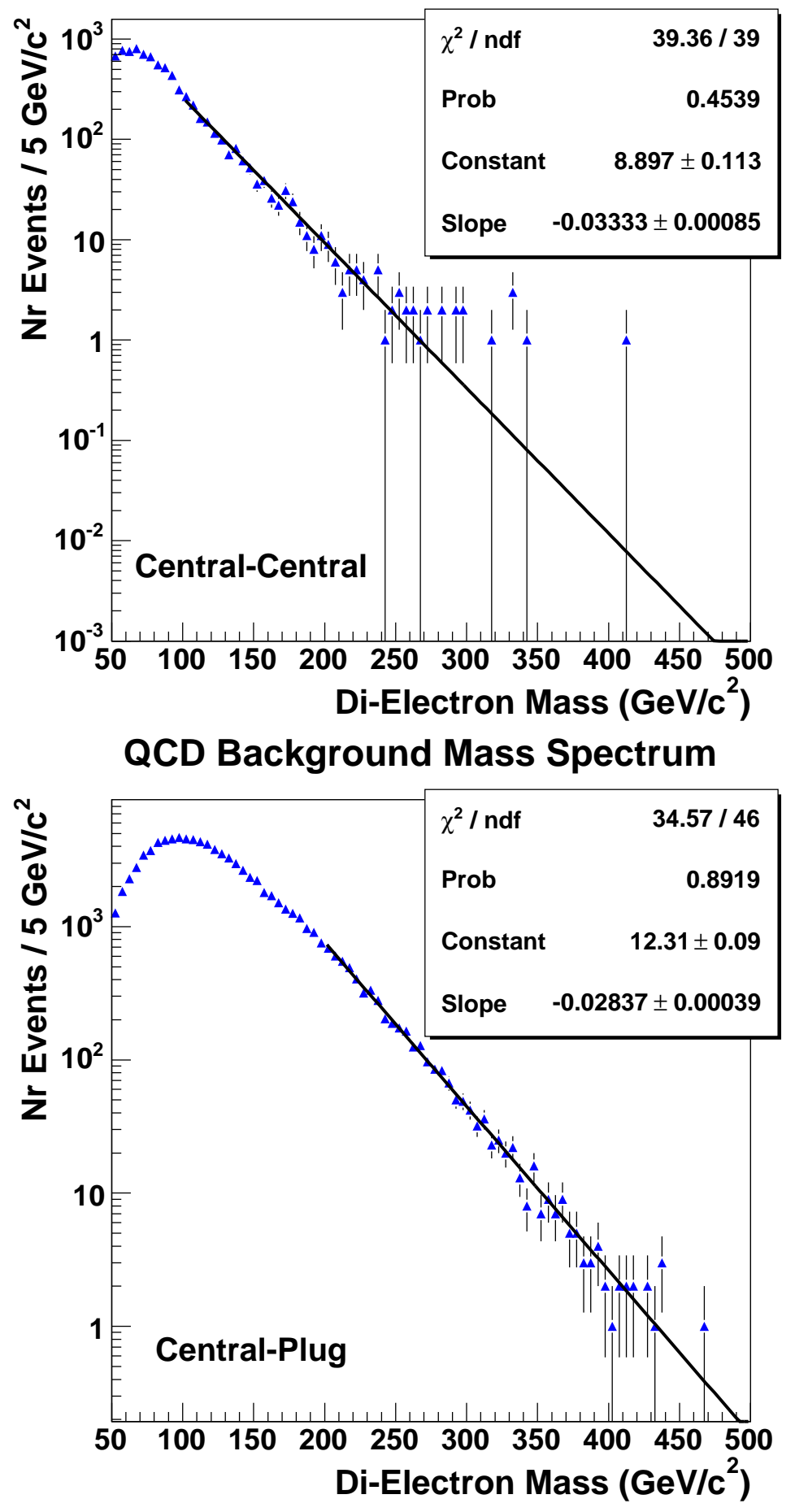

Figure 6.5: The estimated shape of the jet background fitted with an exponential of the form $e^{a+b x}$, with $a$ being the constant term and $b$ being the slope term, in a range of $150 \leq M_{e e} \leq 350 \mathrm{GeV} / c^{2}$ for the central-central and central-plug channels. The insets show the fit parameters together with the fit $\chi^{2}$ and probability. 


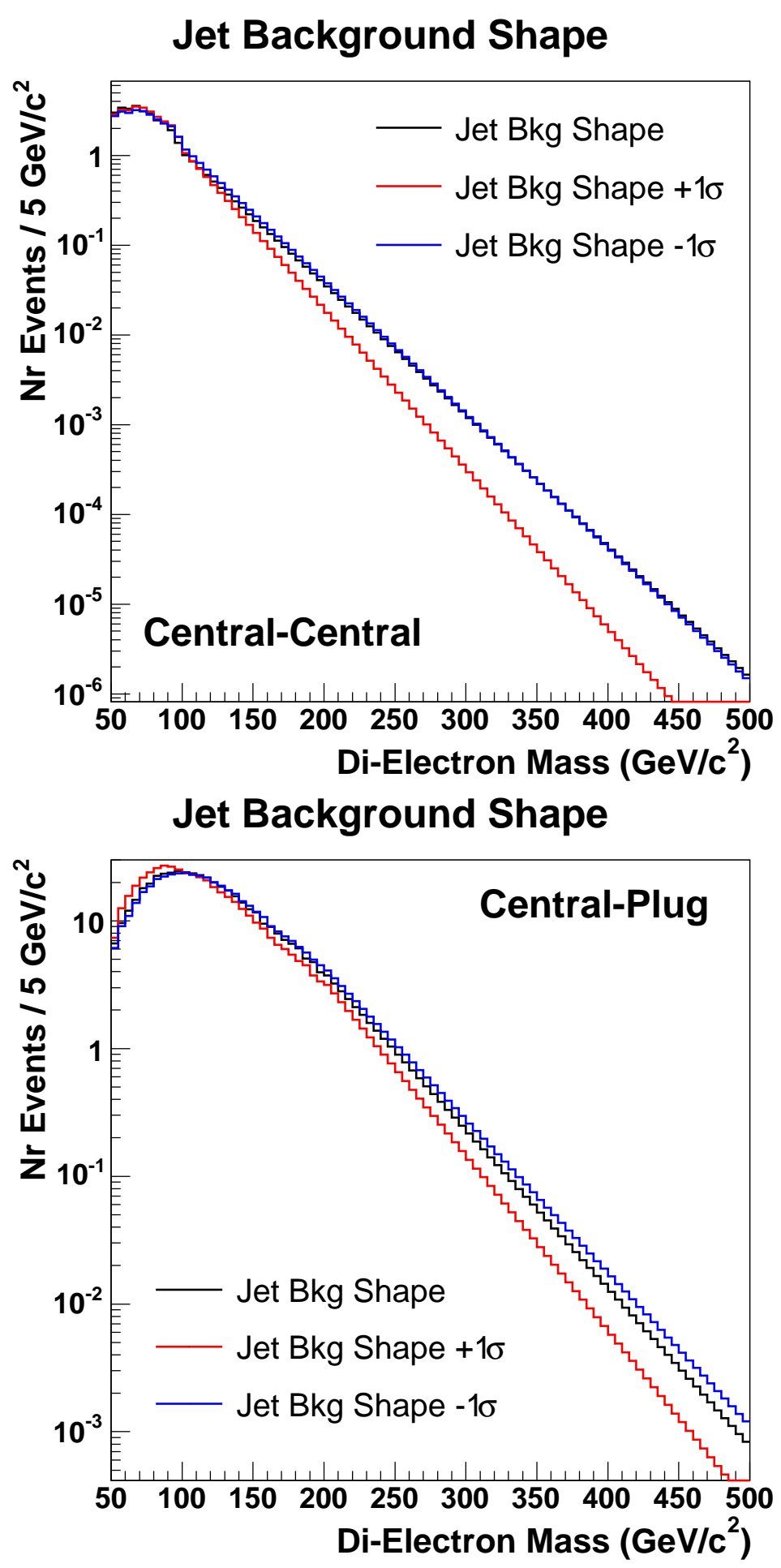

Figure 6.6: The estimated shape of the jet background, normalised to the estimated number of jet events, with the $\pm 1 \sigma$ uncertainties on the shape. 


\begin{tabular}{|c|c|c|c|}
\hline \multirow{2}{*}{ Process } & \multirow{2}{*}{$\sigma . B r(\mathrm{pb})$} & \multicolumn{2}{|c|}{$N_{\text {expected }}$} \\
\cline { 3 - 4 } & & $\mathrm{CC}$ & $\mathrm{CP}$ \\
\hline$Z \rightarrow \tau^{+} \tau^{-}$ & $325 \pm 5$ & $25.1 \pm 0.6$ & $31.3 \pm 0.7$ \\
$t \bar{t}$ & $6.7 \pm 0.5$ & $3.3 \pm 0.3$ & $1.9 \pm 0.1$ \\
$W W$ & $13.25 \pm 0.25$ & $14.5 \pm 0.4$ & $16.3 \pm 0.4$ \\
$W Z$ & $3.96 \pm 0.06$ & $6.9 \pm 0.2$ & $8.3 \pm 0.2$ \\
$W+\gamma$ & $19.3 \pm 1.4$ & $2.0 \pm 0.15$ & $36.4 \pm 2.7$ \\
$\gamma \gamma$ & 185.8 & $23.2 \pm 2.7$ & $46.1 \pm 5.4$ \\
\hline
\end{tabular}

Table 6.11: Theoretically expected cross-sections for the various electroweak backgrounds, together with the number of events expected in the data for $819 \mathrm{pb}^{-1}$. The numbers for the di-photon background are also included. The errors on the number of events also include contributions from the luminosity, acceptance and material uncertainties.

events and any new physics signal present in these channels of a large enough size to have a detectable effect on the cross-check would have already been noticed in the analyses dedicated to these channels.

For each sample the Standard Model backgrounds are estimated using the normal methods and this estimate is then compared to the observed data. The two samples are referred to as the 'anti-Phoenix' and the 'anti-conversion' samples respectively. Of the two samples, the anti-conversion sample offers the better cross-check as it has the least fraction of Drell-Yan events and has no overlap with the normally selected sample. The anti-Phoenix sample is however closer to the selection requirements and so is included as a sanity check. The anti-Phoenix selection requires that one electron passes the CEM CC selection cuts of table 4.2 and one electron passes the PEM selection cuts of table 4.3. Furthermore it imposes the additional requirements that the plug electron satisfies $1.2<\eta_{P E S}<2.0$ and has no associated Phoenix track. In addition the silicon detector is required to have been functioning during the data taking, which reduces the amount of available data to $339 \mathrm{pb}^{-1}$. The silicon and $\eta$ requirements ensure that the plug electron had the opportunity to have a Phoenix track. Jet background events will often not have an associated Phoenix track since jet background events typically have multiple lower momentum tracks which make it difficult to match a single high- $p_{T}$ track to silicon hits in the detector. Therefore these selection requirements will produce a sample with enhanced background levels. The conversion cut on the central leg is not applied to increase statistics in this sample.

The anti-conversion sample requires that the two electrons pass the same selection cuts as normal, with the exception that central leg is required to fail the conversion cut, rather than pass it. Again this will give a sample with enhanced background levels. As can be seen in figures 6.7 and the 6.8, the data agree well with the background estimates in both of the background enriched samples, giving confidence in the background estimates. 

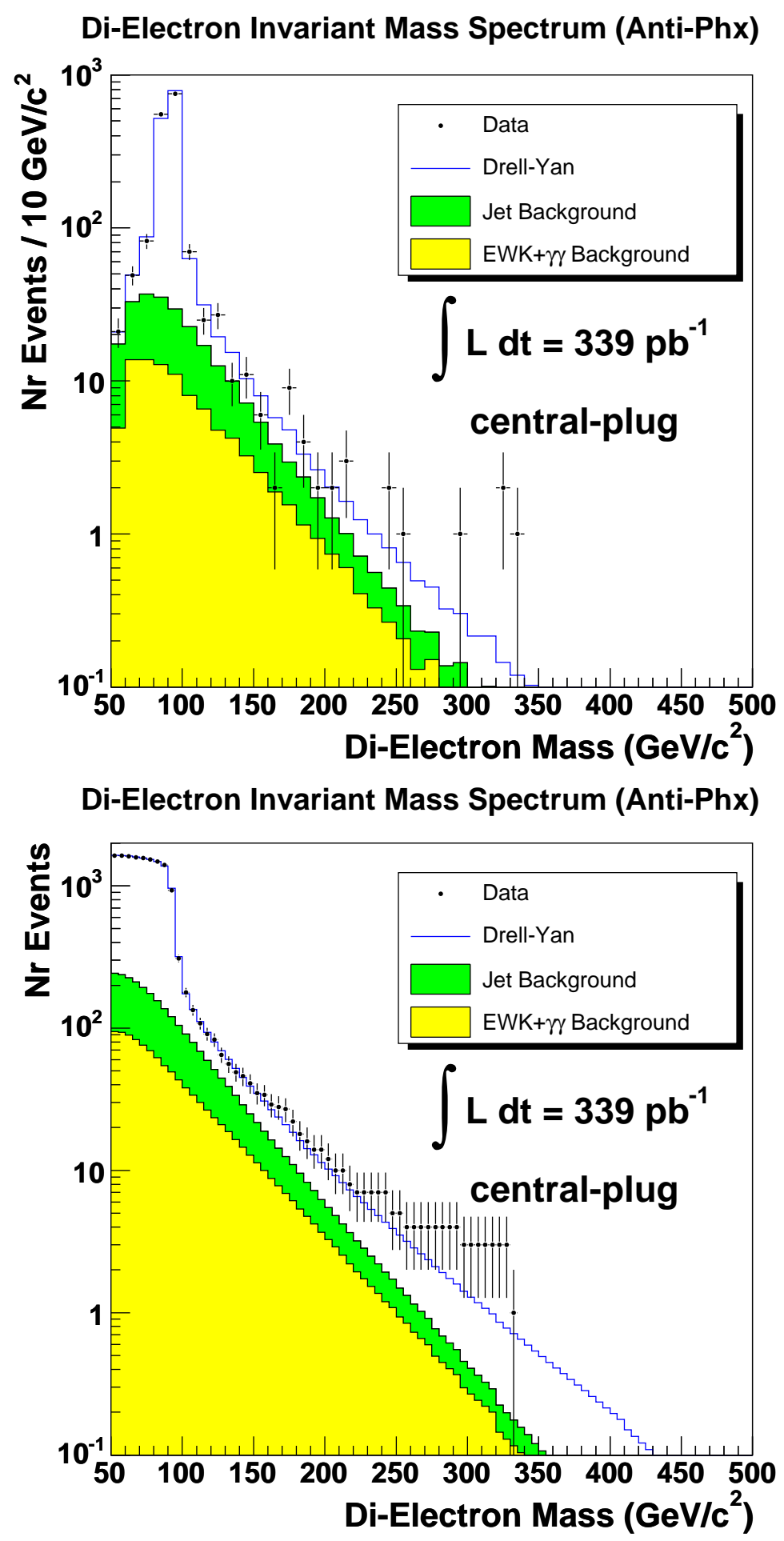

Figure 6.7: The di-electron mass spectrum for central-plug events selected using the anti-Phoenix selection requirements detailed in section 6.5, together with the estimated background. The upper plot shows the mass spectrum and the lower plot shows the integral of the mass spectrum which is more sensitive to disagreements such as a constant over or underestimate in the high mass tail. 

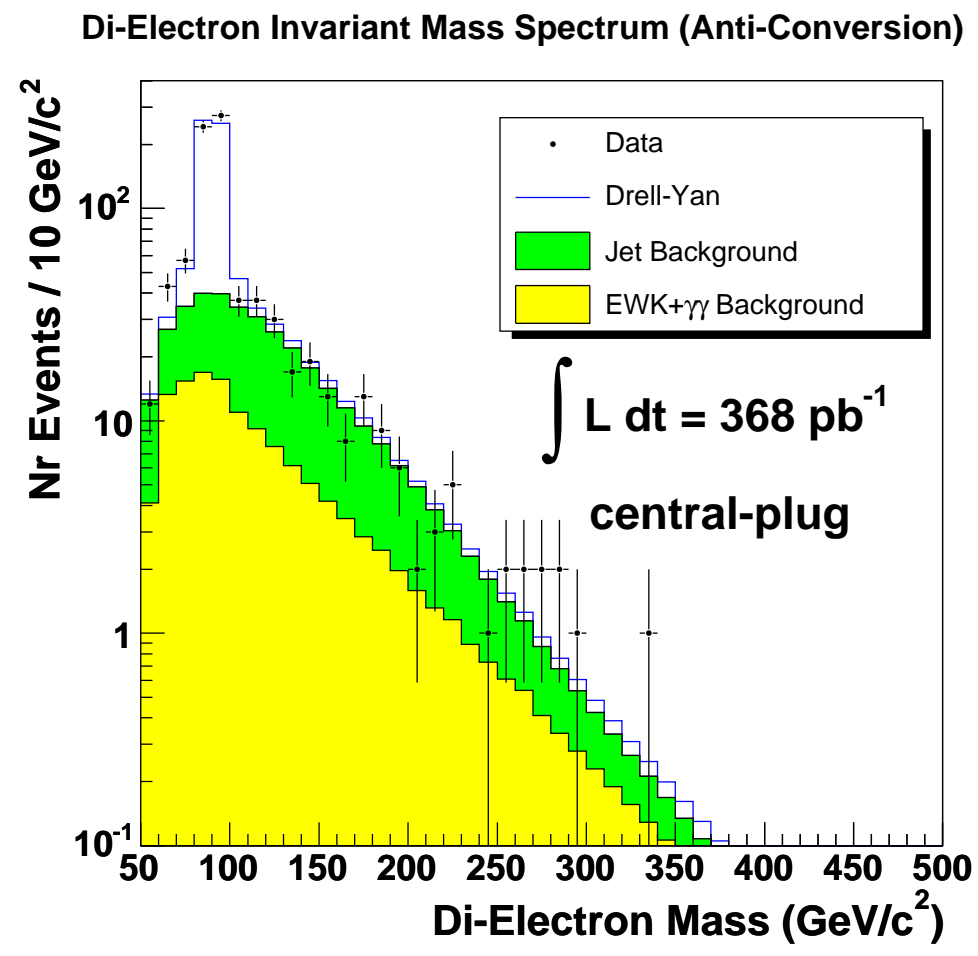

\section{Di-Electron Invariant Mass Spectrum (Anti-Conversion)}

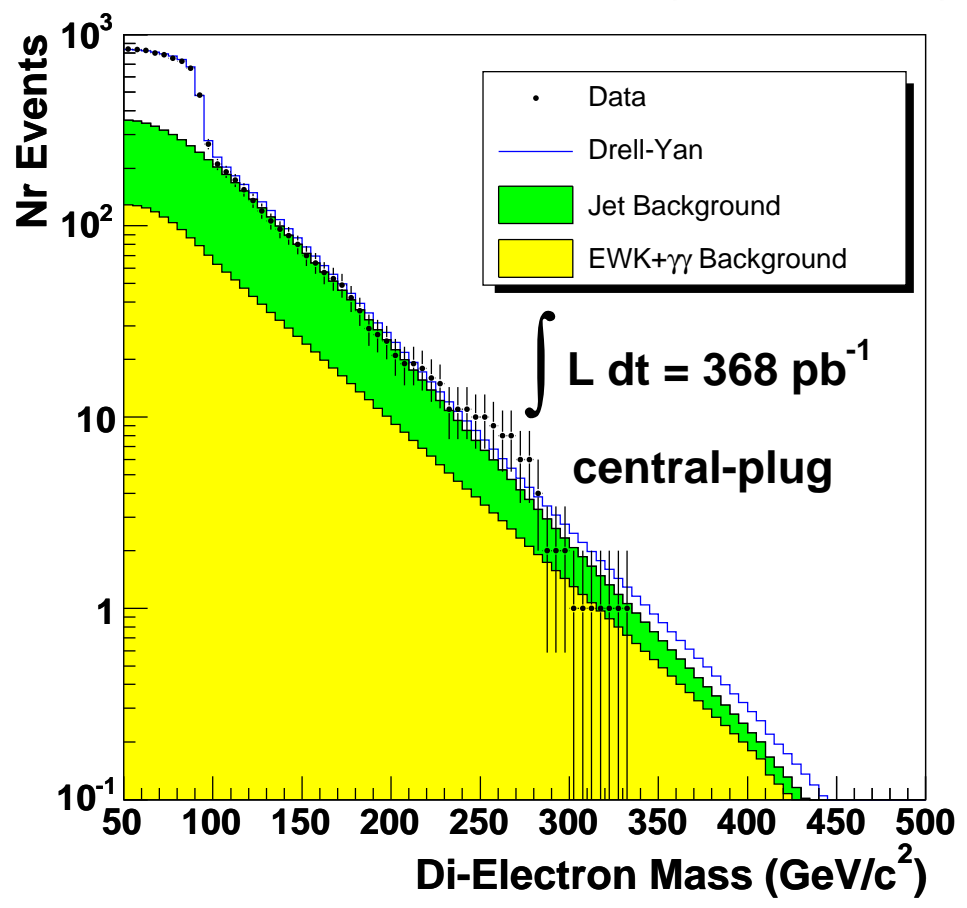

Figure 6.8: The di-electron mass spectrum for central-plug events selected using the anti-conversion selection requirements detailed in section 6.5, together with the estimated background. The upper plot shows the mass spectrum and the lower plot shows the integral of the mass spectrum which is more sensitive to disagreements such as a constant over or underestimate in the high mass tail. 


\section{Chapter 7}

\section{Results}

After the selection cuts and background estimates were fixed, the data in the signal region above $M_{e e}=150 \mathrm{GeV} / c^{2}$ were examined. The measured high mass di-electron mass spectra for the central-central and central-plug channels are shown in figure 7.1 and the combined mass spectrum is shown in figure 7.2. The number of events in each channel at high mass is summarised in table 7.1. In the central-central channel, events are required to have two electrons in the central calorimeter, both with $E_{T} \geq$ $25 \mathrm{GeV}$ and $|\eta| \leq 1.1$. In the central-plug channel, events are required to have one electron in the central calorimeter with $E_{T} \geq 25 \mathrm{GeV}$ and $|\eta| \leq 1.1$ and one electron in the plug calorimeter with $E_{T} \geq 25 \mathrm{GeV}$ and $1.2 \leq|\eta| \leq 3.0$. The selection requirements are discussed in more detail in chapter 4. The background from SM Drell-Yan events is estimated using a $Z^{0} / \gamma^{*}$ Monte Carlo simulation normalised to the data in the regions of $76 \leq M_{e e} \leq 106 \mathrm{GeV} / c^{2}$ and $81 \leq M_{e e} \leq 101 \mathrm{GeV} / c^{2}$ for the central-central and central-plug channels respectively. The jet background shape is estimated from a sample of di-jet events where one of the jets has faked an electron and the resulting mass spectrum is normalised by extrapolating from events in which the electrons are not isolated. The contributions from $\gamma \gamma, t \bar{t}, \tau^{+} \tau^{-}, W W, W Z$ and $W+\gamma$ events are estimated from Monte Carlo simulation normalised to the theoretically expected cross-section. All these background estimates are discussed in more detail in chapter 6 .

The measured mass spectra can be used to search for, and place limits on, new physics processes. The search and limit setting procedures are done separately and use different techniques. Evidence for new physics in the di-electron channel is searched for using a simple model-independent frequentist method which primarily assumes that the width of the resonance of the new physics process is dominated by the resolution of the detector. The method is explained in more detail in section 7.1. Limits are set on specific models using a Bayesian binned likelihood approach with predicted signal shapes and acceptances. The limit setting method is explained in more detail in section 7.2 .

The new physics search does not involve measuring a cross-section and therefore is only affected by the uncertainties on the background distributions, which are represented by the blue bands on figures 7.1 and 7.2 . When setting a cross-section limit the 


\begin{tabular}{|c|c|c|c|}
\hline \multirow{2}{*}{ Source } & \multicolumn{3}{|c|}{ Number of Events with $M_{e e} \geq 200 \mathrm{GeV} / c^{2}$} \\
\cline { 2 - 4 } & Central-Central & Central-Plug & Total \\
\hline \hline Data & 63 & 124 & 187 \\
\hline$Z^{0} / \gamma^{*} \rightarrow e^{+} e^{-}$Bkg. & $66.7 \pm 5.4$ & $77.9 \pm 7.7$ & $144.6 \pm 12.3$ \\
Jet Bkg. & $0.2 \pm 0.2$ & $28.1 \pm 11.2$ & $28.3 \pm 11.2$ \\
$\gamma \gamma$ Bkg. & $1.0 \pm 0.2$ & $3.5 \pm 0.4$ & $4.5 \pm 0.6$ \\
EWK Bkg. & $1.4 \pm 0.1$ & $4.7 \pm 0.7$ & $6.0 \pm 0.7$ \\
\hline Total Bkg. & $69.3 \pm 5.4$ & $114.1 \pm 13.6$ & $183.4 \pm 16.6$ \\
\hline
\end{tabular}

Table 7.1: The number of data and background events with $M_{e e} \geq 200 \mathrm{GeV} / c^{2}$ for both the central-central and the central-plug channels.

result is also affected by the uncertainties on the selection efficiency and luminosity, in addition to the uncertainties on the background distributions. The sources of the systematic uncertainties affecting this analysis are briefly summarised below as well as in table 7.2. More details on the source of the uncertainty on a given quantity is available in the discussions of the individual quantity in the previous chapters.

- Systematic Uncertainty on Energy Scale and Resolution of the Monte Carlo Simulation

The uncertainties on the energy scales of the central and plug calorimeters in the Monte Carlo simulation are both $1 \%$. The uncertainty on the Monte Carlo energy resolution is $0.7 \%$ in the central calorimeter and $0.5 \%$ in the plug calorimeter. More information on the origin of these uncertainties can be found in sections 4.1 .2 and 4.2 .2 .

\section{- Parton Distribution Function Uncertainties}

Monte Carlo generated events are re-weighted with each of the 40 PDF sets obtained by varying the 20 eigenvectors which make up the CTEQ6M PDFs by $\pm 1 \sigma$. The re-weighted results are then compared to the results obtained using the nominal CTE6QM PDFs to obtain the relative change in the background prediction and signal acceptance due to the PDF uncertainties. This is described in more detail in section 5.2 .

\section{- Uncertainties on the Background Expectations}

The uncertainty on the normalisation of the SM Drell-Yan Monte Carlo simulation is determined to be $3.8 \%$ in section 6.1. The jet background has an $85.8 \%$ normalisation uncertainty in the central-central channel and a $32.0 \%$ uncertainty in the central-plug channel. This normalisation uncertainty is obtained from the statistical uncertainty on the method combined in quadrature with the systematic uncertainty obtained from varying the parameters of the method and is described in section 6.2.1. The uncertainty on shape of the jet background is evaluated by re-weighting di-jet events in which one of the jets has faked an 
electron signature by the likelihood for the other jet to fake an electron. This is described in more detail in section 6.2.2. Other backgrounds are sufficiently small so that any uncertainty on them has an insignificant effect on the total background uncertainty.

\section{- Initial State Radiation}

The parameters in the Monte Carlo simulation related to the ISR calculation were changed to be half and then double the default values. This study resulted in a $4 \%$ uncertainty being applied to the acceptance due to the ISR uncertainty and is summarised in section 5.2 .

\section{- Luminosity}

As stated in section 2.2.5 the uncertainty on the luminosity is $6 \%$. This results in a $6 \%$ uncertainty on the extracted cross-section limits. In addition this also leads to a small change in the expected background as the di-photon and electroweak backgrounds are normalised using the luminosity of the data. However, this effect is negligible compared to other uncertainties.

\section{- Electron ID Efficiency}

The uncertainty on the electron ID efficiency at high mass is taken as $2 \%$ for both electrons in the CEM and PEM. The uncertainty on the efficiency scale factor is $0.2 \%$ and $0.3 \%$ for CEM and PEM electrons respectively. More details on the sources of these uncertainties can be found in section 5.1 .

\subsection{New Physics Search}

The primary purpose of this analysis is to search for new physics, in addition to the secondary goal of placing limits on existing models. The new physics signature searched for is a narrow resonance in the di-electron mass spectrum in the range of $150 \leq M_{e e} \leq 950 \mathrm{GeV} / c^{2}$ and it is assumed that the physics process adds incoherently to the Standard Model processes, so it always gives an excess over the Standard Model. The search is otherwise model-independent. In order to ensure the result is statistically meaningful, the method and all the arbitrary parameters, such as the search range, are chosen before looking at the signal region of the data. No modification is allowed to the method once the signal region is unblinded.

The search method is performed as follows. First at $1 \mathrm{GeV} / c^{2}$ intervals along the mass spectrum starting at $150 \mathrm{GeV} / c^{2}$, the number of observed data events and the expected background in a mass window centred at that mass point is calculated. The mass window is chosen to be $4.8+0.044 \times M_{e e} \mathrm{GeV} / c^{2}$, which is the width of a narrow resonance in the CDF detector, as this maximises the sensitivity to such a resonance in pseudo-experiments. Then, using Poisson statistics, the probability for the expected background to fluctuate to the level of the data or higher is calculated. This probability 


\begin{tabular}{|c|c|c|c|c|c|}
\hline & \multirow{2}{*}{ Quantity } & \multicolumn{3}{|c|}{ Measurements Affected } \\
\cline { 3 - 6 } & & \multicolumn{2}{|c|}{ Search } & \multicolumn{2}{|c|}{ Limits } \\
\cline { 3 - 6 } & & $\mathrm{CC}$ & $\mathrm{CP}$ & $\mathrm{CC}$ & $\mathrm{CP}$ \\
\hline Luminosity & $2 \%$ & $\mathrm{X}$ & $\mathrm{X}$ & $\sqrt{ }$ & $\sqrt{ }$ \\
High Mass Eff. (CEM) & $2 \%$ & $\mathrm{X}$ & $\mathrm{X}$ & $\sqrt{ }$ & $\sqrt{ }$ \\
High Mass Eff. (PEM) & $\mathrm{X}$ & $\mathrm{X}$ & $\mathrm{X}$ & $\sqrt{ }$ \\
Initial State Radiation & $4 \%$ on acceptance & $\mathrm{X}$ & $\mathrm{X}$ & $\sqrt{ }$ & $\sqrt{ }$ \\
\hline PDF Uncertainties & see text & $\sqrt{ }$ & $\sqrt{ }$ & $\sqrt{ }$ & $\sqrt{ }$ \\
MC Energy Scale (CEM) & $1 \%$ & $\sqrt{ }$ & $\sqrt{ }$ & $\sqrt{ }$ & $\sqrt{ }$ \\
MC Energy Scale (PEM) & $1 \%$ & $\mathrm{X}$ & $\sqrt{ }$ & $\mathrm{X}$ & $\sqrt{ }$ \\
MC Energy Res. (CEM) & $0.7 \%$ & $\sqrt{ }$ & $\sqrt{ }$ & $\sqrt{ }$ & $\sqrt{ }$ \\
MC Energy Res. (PEM) & $0.5 \%$ & $\mathrm{X}$ & $\sqrt{ }$ & $\mathrm{X}$ & $\sqrt{ }$ \\
\hline$e^{+} e^{-}$Jet Bkg. Norm. (CC) & $85.8 \%$ & $\sqrt{ }$ & $\mathrm{X}$ & $\sqrt{ }$ & $\mathrm{X}$ \\
$e^{+} e^{-}$Jet Bkg. Norm. (CP) & $32.0 \%$ & $\mathrm{X}$ & $\sqrt{ }$ & $\mathrm{X}$ & $\sqrt{ }$ \\
$e^{+} e^{-}$Jet Bkg. Shape (CC) & see text & $\sqrt{ }$ & $\mathrm{X}$ & $\sqrt{ }$ & $\mathrm{X}$ \\
$e^{+} e^{-}$Jet Bkg. Shape (CP) & see text & $\mathrm{X}$ & $\sqrt{ }$ & $\mathrm{X}$ & $\sqrt{ }$ \\
$Z^{0} / \gamma^{*} \rightarrow e^{+} e^{-}$MC Norm. & $3.8 \%$ & $\sqrt{ }$ & $\sqrt{ }$ & $\sqrt{ }$ & $\sqrt{ }$ \\
\hline \hline
\end{tabular}

Table 7.2: Summary of systematic uncertainties affecting the new physics search and limits for each channel with a $\sqrt{ }$ indicating the channel is affected by the uncertainty and X indicating it is not. Uncertainties that are listed as "see text" are uncertainties which can not easily be expressed as a single number. Note that while the luminosity uncertainty does affect the new physics search, the effect is negligible. 
Di-Electron Invariant Mass Spectrum

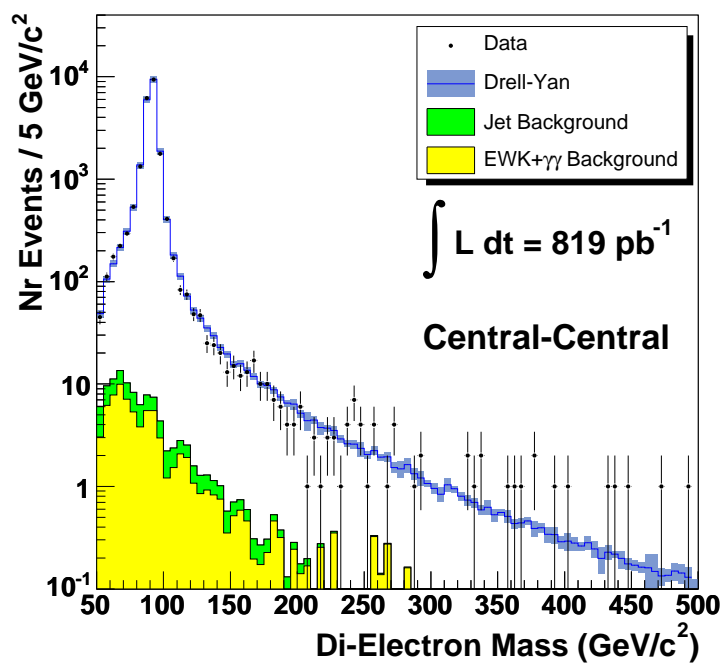

Integral Plot of the Di-Electron Mass Spectrum

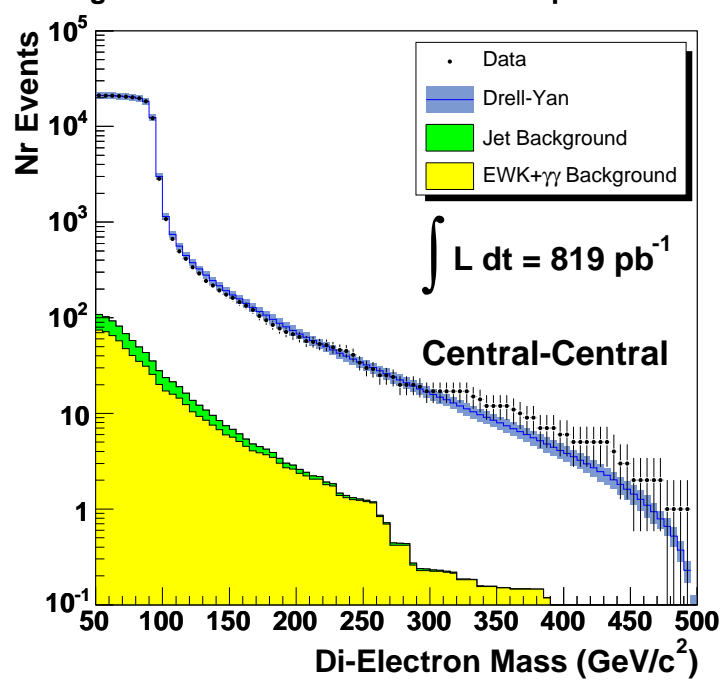

Di-Electron Invariant Mass Spectrum

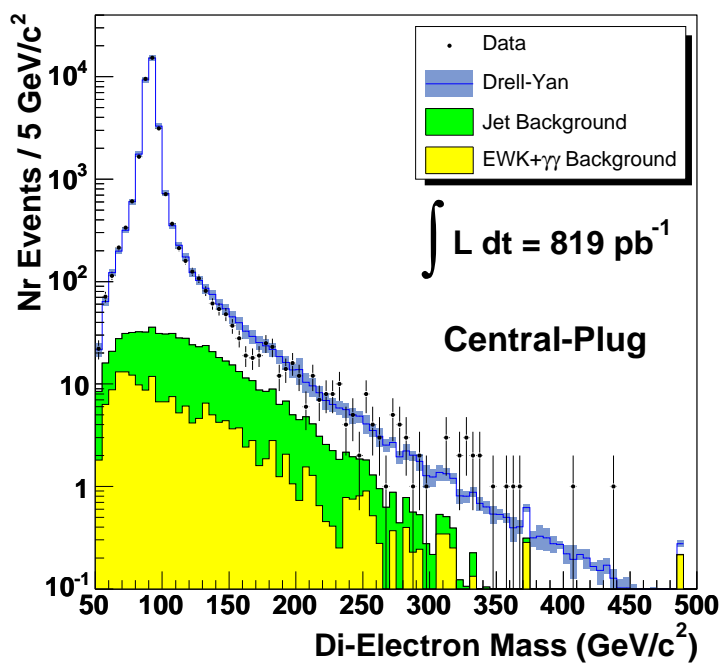

Integral Plot of the Di-Electron Mass Spectrum

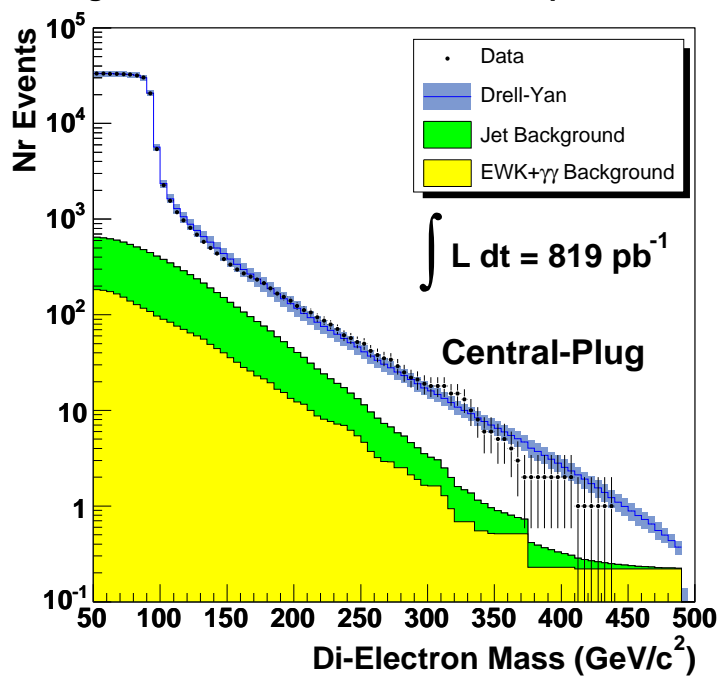

Figure 7.1: The di-electron mass spectra for the central-central and central-plug channels. The bottom plots are integral plots of the top plots which are more sensitive to disagreements such as a constant over or underestimate in the high mass tail. 


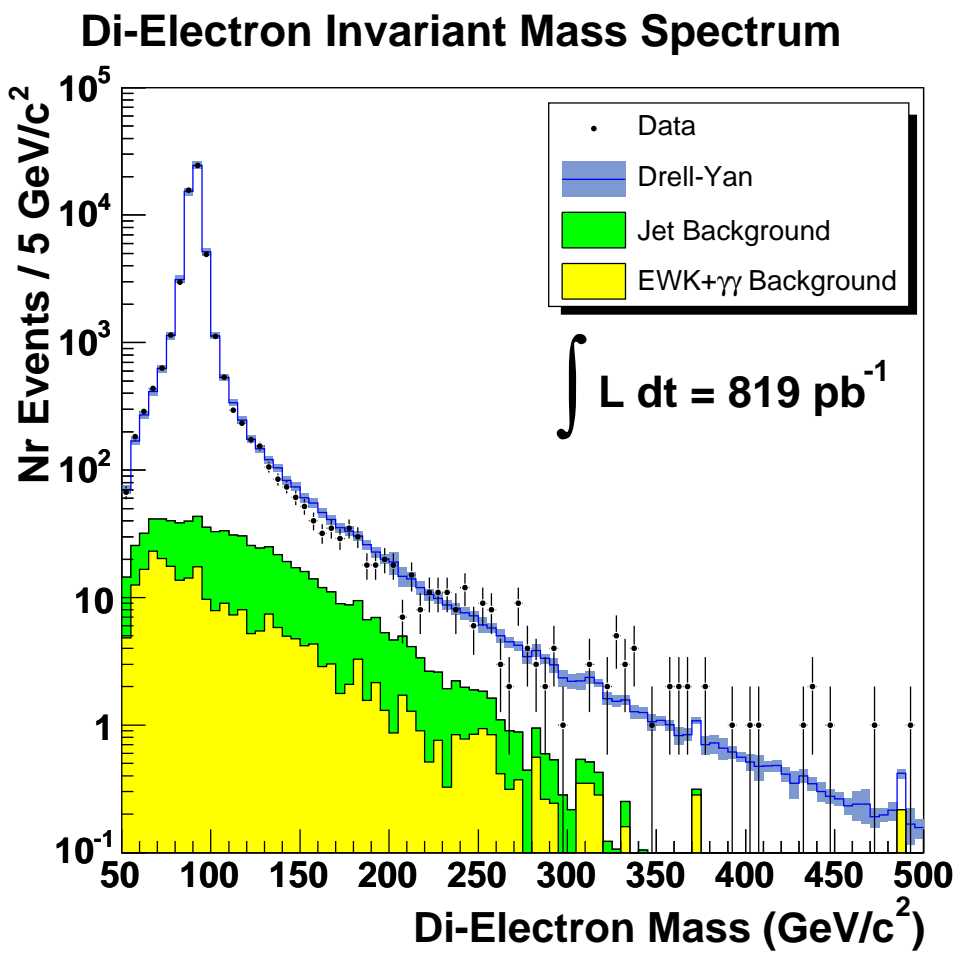

Integral Plot of the Di-Electron Mass Spectrum

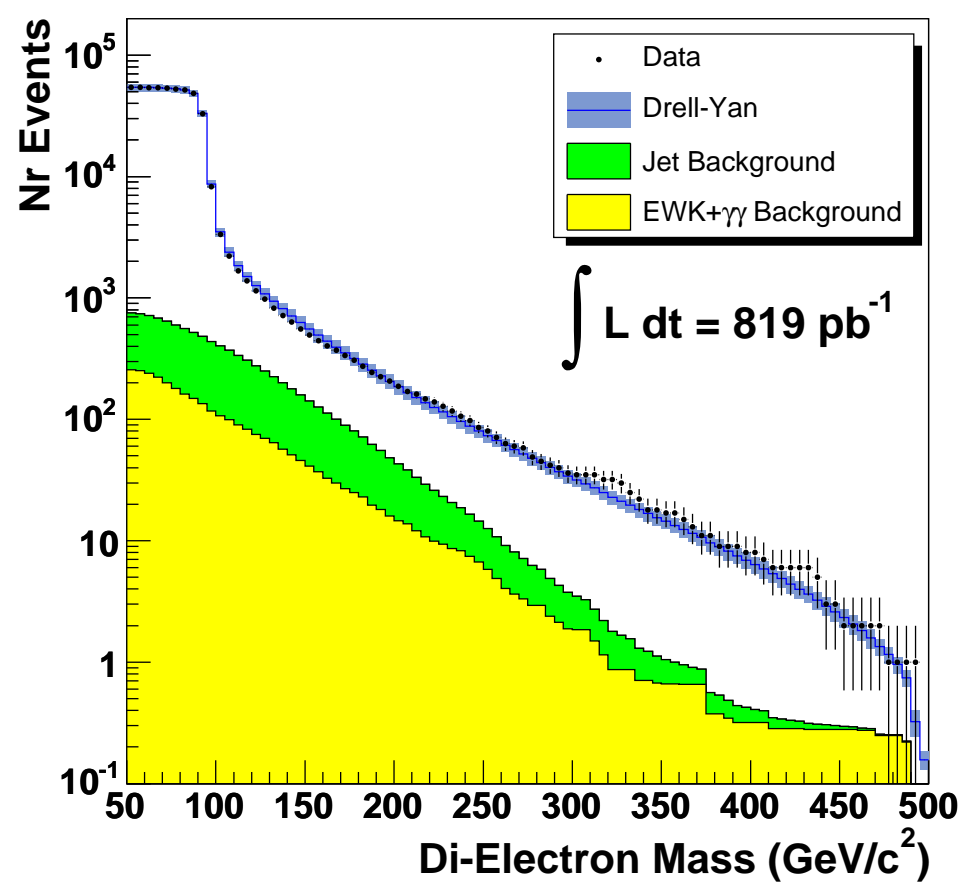

Figure 7.2: The di-electron mass spectrum for the central-central and central-plug channels combined. The bottom plot is an integral plot of the top plot which is more sensitive to disagreements such as a constant over or underestimate in the high mass tail. 
is referred to as the p-value. The uncertainty on the background estimate is incorporated by integrating the probability over all possible background values weighted by a normalised Gaussian with mean and sigma equal to the background expectation and uncertainty, as recommended by the CDF statistics committee [59]. The p-values for the central-central and central-plug channels are combined multiplicatively to give the total p-value.

After all the p-values have been calculated, an additional step must be taken to determine their compatibility with the null hypothesis that there is no new physics signal present. This is because the mass spectrum is scanned from $150 \mathrm{GeV} / c^{2}$ to $950 \mathrm{GeV} / c^{2}$ in $1 \mathrm{GeV} / c^{2}$ steps giving a total of $800 \mathrm{p}$-value measurements. While the mass window means that neighbouring p-values are highly correlated, and therefore the 800 measurements are not independent, there are still many independent and weakly correlated measurements and a small p-value is increasingly likely to happen by chance alone. To determine how likely it is that a given p-value or lower will occur due to a statistical fluctuation at some mass point during the mass spectrum scan, 20000 pseudo-experiments are constructed. These pseudo-experiments use Poisson statistics to generate a mass spectrum using the expected background distribution as a template. The minimum p-value observed in the scan of each pseudo-experiment's mass spectrum is recorded and the percentage of experiments having a given p-value or lower is summarised in table 17.3. An expectation on the minimum p-value observed in the mass spectrum in the absence of new physics is obtained by taking the median of the minimum p-values observed in the pseudo-experiments. The expected minimum p-value is $5.6 \times 10^{-3}$ and expected range, defined as the range in which the minimum $\mathrm{p}$-value of $68.3 \%$ of pseudo-experiments lie, is $1.4 \times 10^{-3}-1.6 \times 10^{-2}$. The $3 \sigma$ evidence level pvalue is defined as the p-value for which only $0.15 \%$ of the pseudo-experiments observe an equal or lower p-value. This $\mathrm{p}$-value is found to be $1.5 \times 10^{-5}$, and an observed p-value lower than this would be taken as evidence for new physics. The result of the search is shown in figure 7.3. The individual p-value spectrums for the two channels are shown in figure 7.4 as it is interesting to see in which channel a given excess occurs, although only the combined result is statistical meaningful in the context of the new physics search in this analysis. It is important to note that the expected and $3 \sigma$ p-values shown above are only valid for the combined p-values and that the expected and $3 \sigma$ p-values of the individual channels will be different.

The lowest $\mathrm{p}$-value observed in the data is $1.8 \times 10^{-3}$, at a mass of about $330 \mathrm{GeV} / c^{2}$. This p-value or lower occurs in approximately $19 \%$ of the pseudo-experiments and is within the expected range. It is therefore concluded that there is no significant evidence for new physics anywhere in the region of the mass spectrum explored.

\subsection{Limits on New Physics}

Limits are set on the cross-section $\times$ branching ratio $(\sigma . \mathrm{Br})$ to two electrons for neutral massive spin-1 and spin-2 particles at the $95 \%$ confidence level (CL). In order to be 


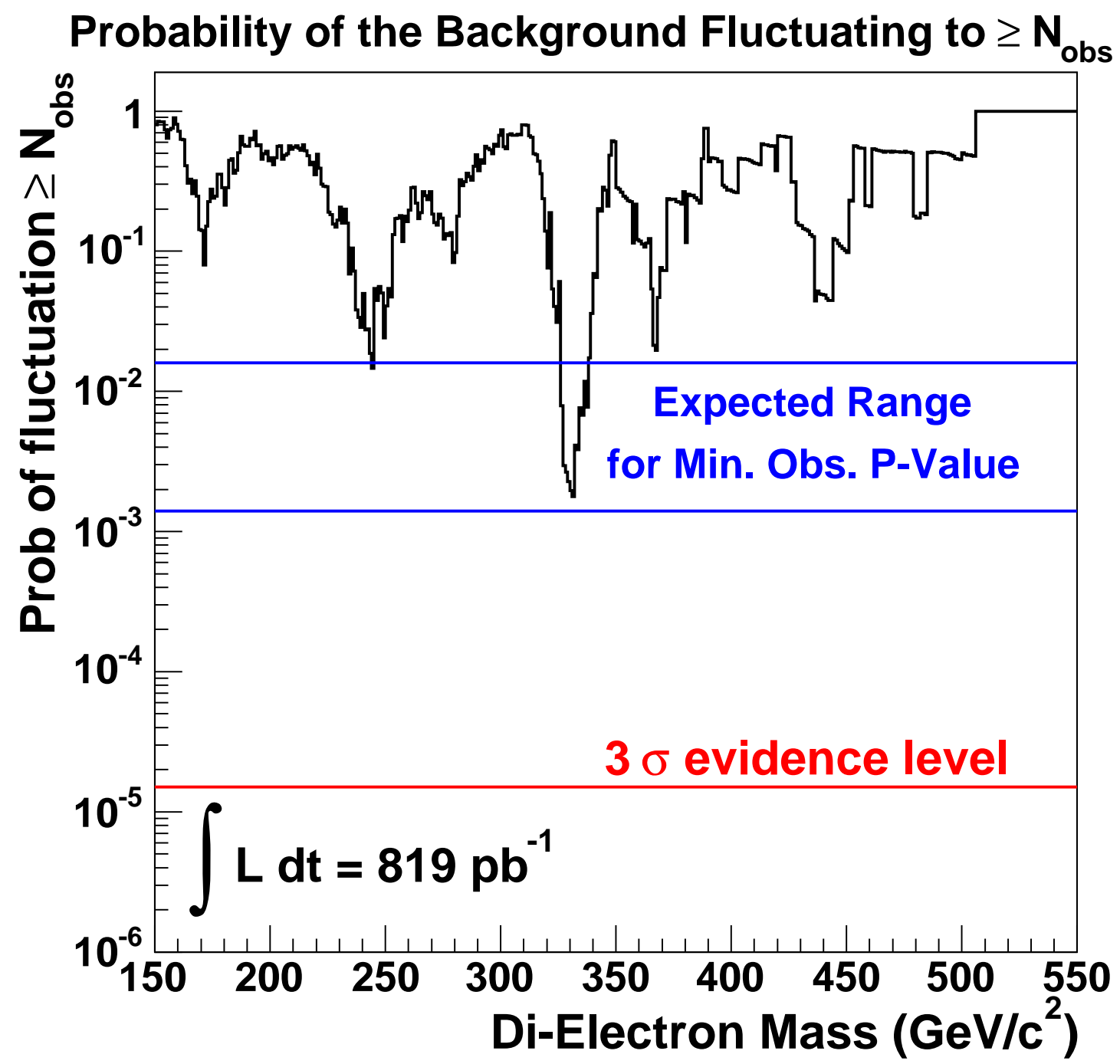

Figure 7.3: The probability of observing a number of events equal to or greater than the number of events actually observed in data, referred to as the p-value, in a window equal to the width of a narrow resonance in the CDF detector, given the estimated background and the absence of new physics for central-central and central-plug channels combined. The range in which the minimum observed p-value is expected to lie in the absence of new physics and the p-value required for $3 \sigma$ evidence for the presence of new physics are also shown. 

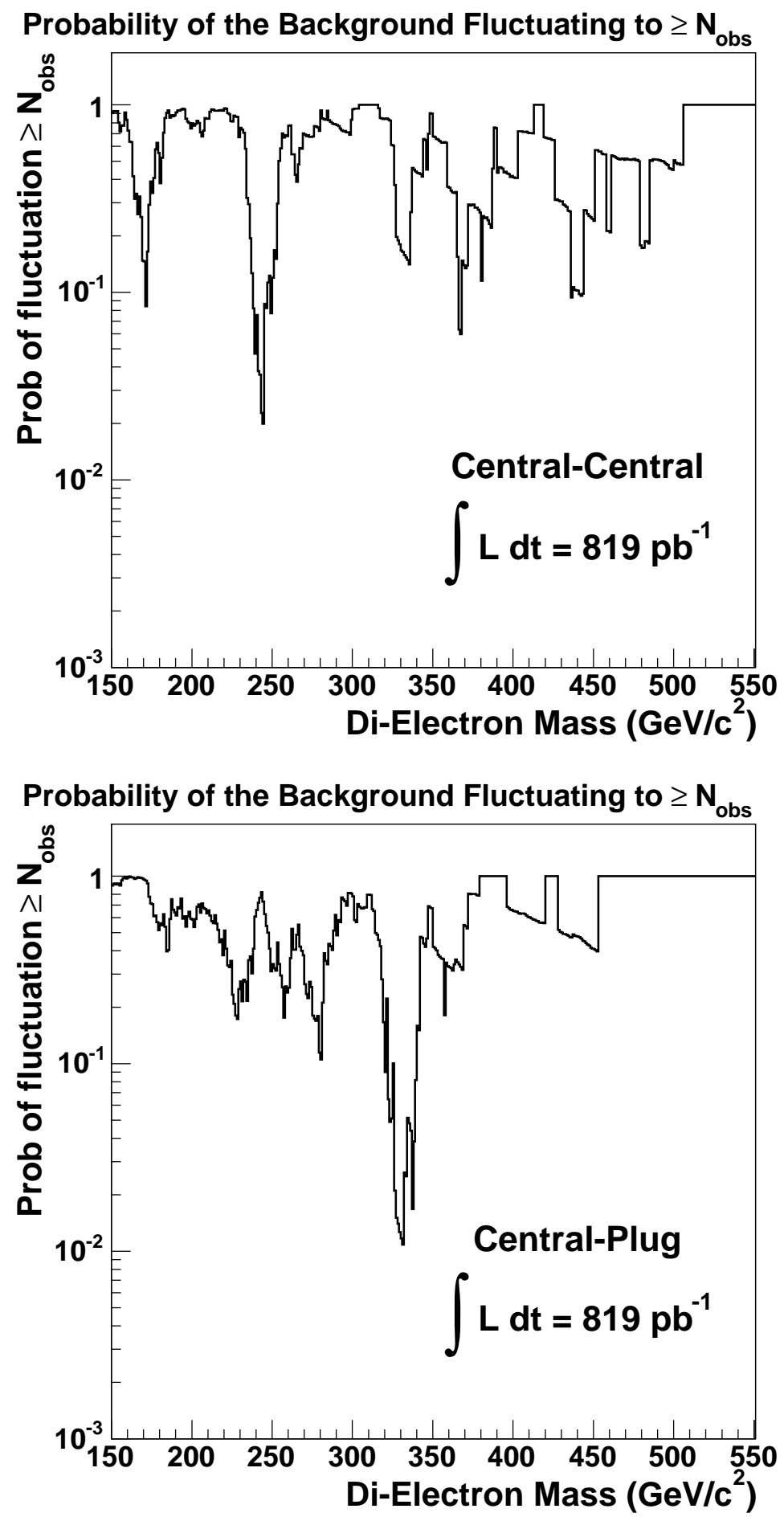

Figure 7.4: The probability to observe a number of events equal to or greater than the number of events actually observed in data, referred to as the p-value, in a window equal to the width of a narrow resonance in the CDF detector, given the estimated background and the absence of new physics for central-central (top) and central-plug (bottom) channels. 


\begin{tabular}{|c|c|}
\hline P-value & \% of Experiments with min prob $\leq \mathrm{p}$-value \\
\hline $1 \times 10^{-2}$ & $69.2 \%$ \\
$1 \times 10^{-3}$ & $11.8 \%$ \\
$1 \times 10^{-4}$ & $1.3 \%$ \\
$1 \times 10^{-5}$ & $0.1 \%$ \\
\hline
\end{tabular}

Table 7.3: The percentage of pseudo-experiments in which a given p-value or lower is observed at any mass point in the mass spectrum from $150-950 \mathrm{GeV} / c^{2}$.

compatible with other CDF analyses, the cross-section is calculated in a mass window of $\pm 10 \%$ of the on-shell mass of the particle.

\subsubsection{Procedure for Setting Limits on New Physics Processes}

As in the previously published analysis [10, a Bayesian binned likelihood method is used to calculate the limits. The bin contents are treated using Poisson statistics. The likelihood as a function of cross-section $(\sigma)$ can be written as

$$
\begin{aligned}
L(\sigma) & =\prod_{i=1}^{N_{\text {bins }}} \frac{\mu(\sigma)_{i}^{N_{i}^{d}} e^{-\mu(\sigma)_{i}}}{N_{i}^{d} !} \\
\mu(\sigma)_{i} & =A \epsilon \mathcal{L} \sigma N_{i}^{s} / N_{\text {tot }}^{s}+N_{i}^{b}
\end{aligned}
$$

where $N_{i}^{d}, N_{i}^{b}$ and $N_{i}^{s}$ are the number of data, background and signal events in the $i^{\text {th }}$ bin respectively, $A$ is the acceptance, $\epsilon$ is the efficiency, $\mathcal{L}$ is the luminosity and $N_{\text {tot }}^{s}$ is the total number of signal events passing selection requirements. The $95 \%$ confidence limit is obtained by finding the value of $\sigma_{95}$ for which

$$
\frac{\int_{0}^{\sigma_{95}} L(\sigma) d \sigma}{\int_{0}^{\infty} L(\sigma) d \sigma}=0.95
$$

is true. To combine multiple channels, the individual likelihoods are multiplied together to obtain the combined likelihood, which is treated identically to the single channel likelihood. In order to verify the limit setting framework, the pull of likelihood fit is checked to ensure that is reasonable. This is achieved by constructing 10000 pseudo-experiments, each with a signal cross-section of $1 \mathrm{pb}$. The pull is defined as $\left(\sigma_{f i t}-\sigma_{\text {true }}\right) / \Delta \sigma_{\text {fit }}$ where $\sigma_{f i t}$ is the best fit to the cross-section of the signal present, $\Delta \sigma_{f i t}$ is the uncertainty on the fitted value and $\sigma_{\text {true }}$ is the true cross-section of the signal present. Figure 7.5 shows the pull of the likelihood fit. The pull is approximately a Gaussian with a mean close to zero and a sigma of slightly less than one, which is as expected. 


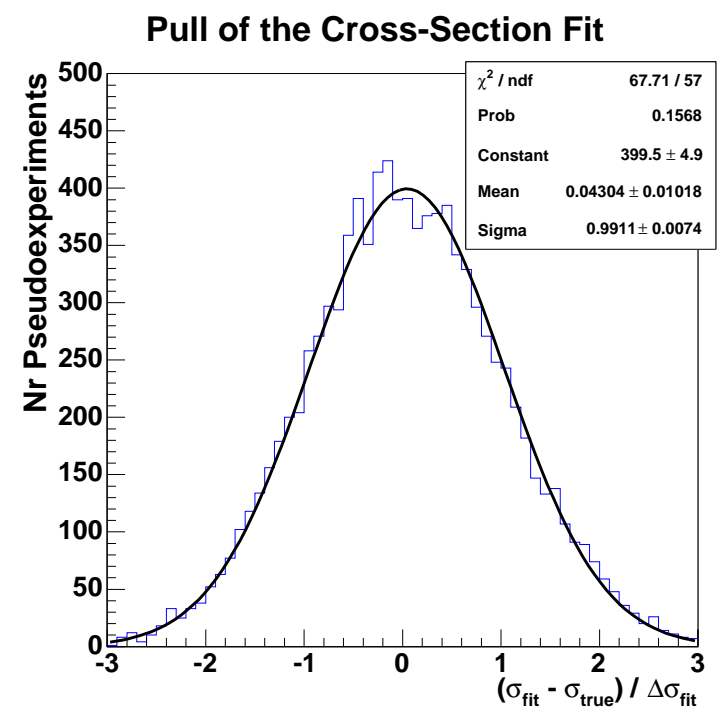

Figure 7.5: The pull of the cross-section fit for a $500 \mathrm{GeV} / c^{2}$ spin- 1 particle in the central-central and central-plug channels combined.

\subsubsection{Procedure for Including Systematic Uncertainties}

To take into account the uncertainties on the quantities and distributions used in the limit calculation, the method used in [10, 60] is followed. There are two steps in the method. The first step is to translate the uncertainty on an input quantity, such as the jet background normalisation or the luminosity, into an uncertainty on the crosssection. The second is to take the resulting total uncertainty on the cross-section, $\Delta \sigma$, and use it to smear the likelihood function to reflect the uncertainty on the crosssection. The likelihood for a particular cross-section is smeared by integrating over all possible cross-sections weighted by a Gaussian with a mean and sigma equal to the given cross-section and its uncertainty, as shown in equation 7.4 .

$$
L^{\text {smear }}(\sigma)=\int_{0}^{\infty} L\left(\sigma^{\prime}\right) \frac{e^{-\frac{\left(\sigma^{\prime}-\sigma\right)^{2}}{2 \Delta \sigma^{2}}}}{\sqrt{2 \pi \Delta \sigma^{2}}} d \sigma^{\prime}
$$

The absolute size of the cross-section uncertainty is not constant, and is taken to have a linear dependence on the cross-section of the form $\Delta \sigma=A \sigma+B$ [60]. To understand the origin of the slope and constant terms it is helpful to think of two uncertainty types: pure cross-section uncertainties and pure signal-like background uncertainties. Pure cross-section uncertainties are uncertainties on quantities that are either directly or inversely proportional to the cross-section, and therefore the percentage uncertainty on the cross-section will be equal to the percentage uncertainty on the given quantity. Hence they have a slope term but no constant term. Examples of these types of uncertainties are the uncertainties on the luminosity measurement and the acceptance 
estimates. To follow the example through, it can easily be seen that a $6 \%$ uncertainty on the luminosity will lead to a $6 \%$ uncertainty on the cross-section.

A pure signal-like background is a background which indistinguishable from the signal and also has the same mass spectrum distribution. In the case of a pure signallike background, an increase in the amount of background present will simply reduce the amount of signal that can be present. This will lead to a constant uncertainty independent of cross-section.

The above two cases are idealised and in practise, an uncertainty will consist of a mixture of both types and will have both a slope and constant term. For example the luminosity is also used to normalise the EWK and di-photon backgrounds, giving it a small constant term. Additionally the jet background does not look exactly like the signal distribution, giving it a small slope term.

To obtain the linear dependence of the cross-section uncertainty due to an uncertainty on an input parameter, the input parameter's uncertainty is first varied by \pm 1 standard deviation to obtain two new background and signal histograms to use as templates for pseudo-experiments. Each pseudo-experiment uses Poisson statistics to generate a mass spectrum using these templates, with a signal normalisation corresponding to a cross-section of $1 \mathrm{pb}$. For each experiment, a fit is performed by maximising the likelihood defined in equation 7.1 to extract the most likely value of the cross-section using the same templates used to generated the mass spectrum. The fit is then re-performed using the nominal signal and background histograms as templates. 10000 pseudo-experiments are produced for each mass point and the difference between the means of the two extracted cross-sections of the 10000 pseudo-experiments is taken as the uncertainty on the cross-section. Then to obtain the uncertainty as a function of cross-section, the process is repeated for signal normalisations corresponding to crosssections of 2, 3, 4 and $5 \mathrm{pb}$ and a linear function is fitted to the resulting distribution. This linear function is then used to obtain $\Delta \sigma$ for a particular cross-section resulting from the given uncertainty. The individual cross-section uncertainties are added in quadrature to obtain the total cross-section uncertainty. The effect of the systematic uncertainties on the likelihood can be seen in figure 7.6 for spin-1 particles, with the main effects being a shift of the maximum to a higher value and a decrease in the rate the likelihood drops off. The effects of the systematic uncertainties are largest at low masses due to the larger uncertainties on the background estimate in the low mass region. At high mass the background estimate does not the observed limit as there are no observed events.

\subsubsection{Expected and Observed Limits}

In order to compare the observed limits to those that would be expected in the absence of new physics, 10000 pseudo-experiments are constructed for each mass point. Each pseudo-experiment uses Poisson statistics to generate a mass spectrum using the expected Standard Model background distribution as a template. A limit on the $\sigma$. Br of a new physics process is then extracted from the mass spectrum in an identical 
Likelihood function for a $200 \mathrm{GeV} / \mathrm{c}^{2} \mathrm{Z}^{\prime}$

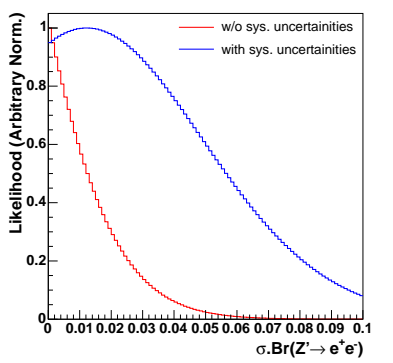

Likelihood function for a $350 \mathrm{GeV} / \mathrm{c}^{2} \mathrm{Z}$

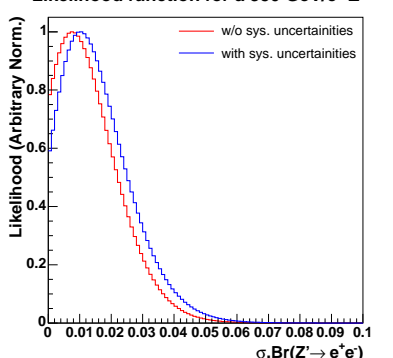

Likelihood function for a $500 \mathrm{GeV} / \mathrm{c}^{2} \mathrm{Z}^{\prime}$

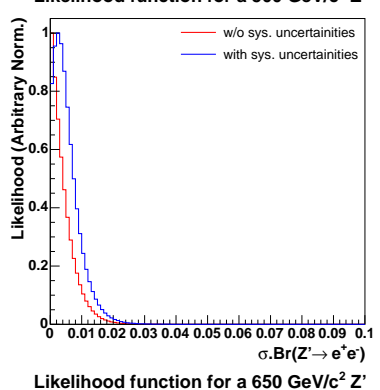

Likelihood function for a $650 \mathrm{GeV} / \mathrm{c}^{2} \mathrm{Z}$

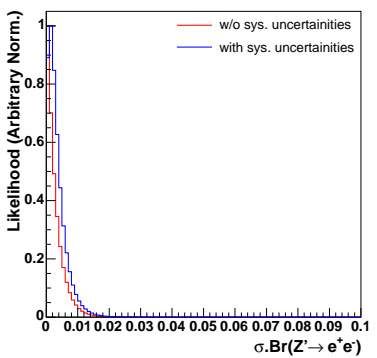

Likelihood function for a $800 \mathrm{GeV} / \mathrm{c}^{2} \mathrm{Z}$

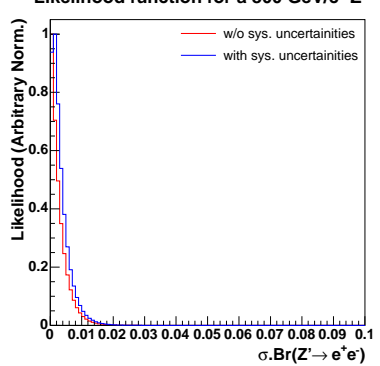

Likelihood function for a $250 \mathrm{GeV} / \mathrm{c}^{2} \mathrm{Z}$

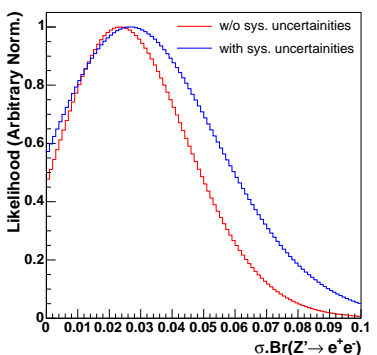

Likelihood function for a $400 \mathrm{GeV} / \mathrm{c}^{2} \mathrm{Z}$

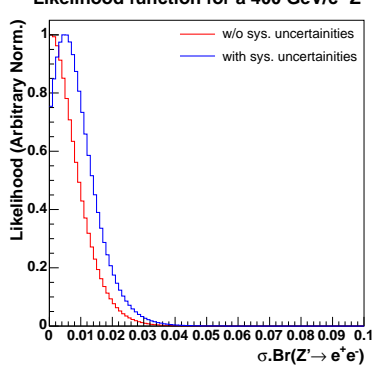

Likelihood function for a $550 \mathrm{GeV} / \mathrm{c}^{2} Z^{\prime}$

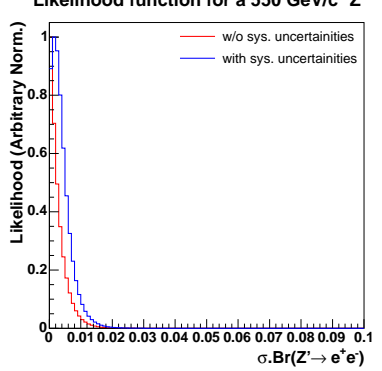

Likelihood function for a $700 \mathrm{GeV} / \mathrm{c}^{2} \mathrm{Z}$

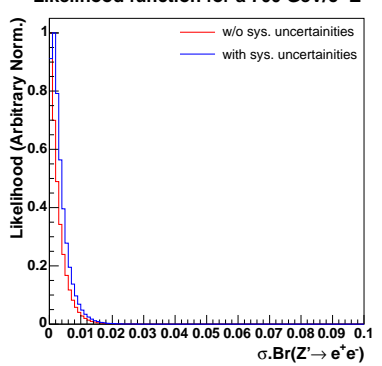

Likelihood function for a $850 \mathrm{GeV} / \mathrm{c}^{2} \mathrm{Z}$

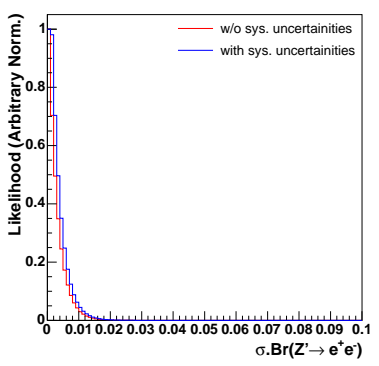

Likelihood function for a $300 \mathrm{GeV} / \mathrm{c}^{2} \mathrm{Z}$

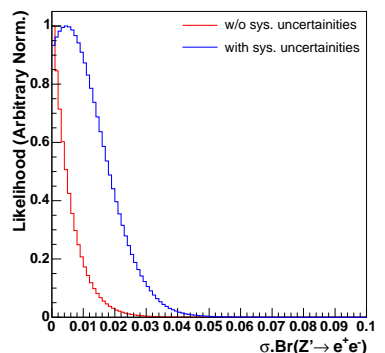

Likelihood function for a $450 \mathrm{GeV} / \mathrm{c}^{2} \mathrm{Z}$

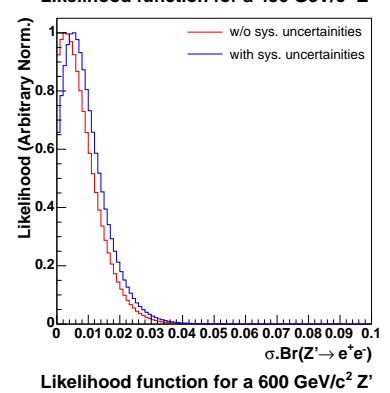

Likelihood function for a $600 \mathrm{GeV} / \mathrm{c}^{2} Z$
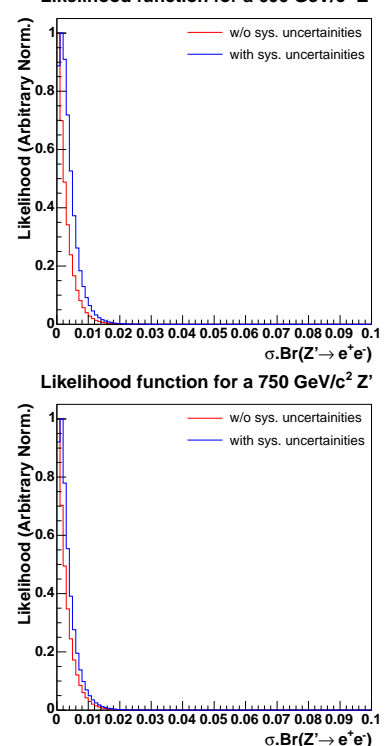

Likelihood function for a $900 \mathrm{GeV} / \mathrm{c}^{2} \mathrm{z}$

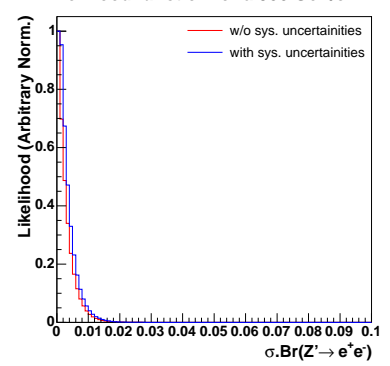

Figure 7.6: Likelihood distributions for spin-1 particles for each mass point with and without including the effects of systematic uncertainties. The two distributions are normalised such that they peak at 1 . The largest effect on the likelihood comes from the background uncertainties which are only significant at low mass where the backgrounds are largest. 
manner to that used for the data. The median of these limits is taken as the expected limit due to its property that $50 \%$ of experiments will be above it and $50 \%$ will be below it. The 1, 2 or 3 sigma confidence bands on the expected limit are obtained by finding the ranges which contain $68.3 \%, 95.4 \%$ or $99.7 \%$ of the limits obtained from the pseudo-experiments. Figures 7.7 and 7.8 show the observed limits together with the expected limits for spin-1 and spin-2 particles. No significant deviation from the expected limit is observed.

\subsubsection{Model Specific Limits}

The $95 \%$ confidence level limits on the $\sigma \cdot \operatorname{Br}\left(X \rightarrow e^{+} e^{-}\right)$of generic spin-1 or spin-2 particles can be used to place mass exclusion limits for specific models. The models on which limits are set on are discussed in section 1.2 . These models are the $E_{6}$ GUT group model, Technicolour and the Randall-Sundrum theory of warped extra dimensions, with the first two predicting new spin-1 particles and the last one predicting a new spin-2 particle. Each model predicts the new particle's $\sigma \cdot \operatorname{Br}\left(p \bar{p} \rightarrow X \rightarrow e^{+} e^{-}\right)$at $\sqrt{s}=1.96 \mathrm{TeV}$ for a given mass of the particle. This allows the mass limit on the particles to be set by finding the point where the predicted $\sigma . \mathrm{Br}$ is no longer excluded.

The predicted $\sigma$. Br as a function of mass of a model is referred to as a model line and is obtained using either the PYTHIA or HERWIG event generators. The PYTHIA event generator is used for all the models predicting spin-1 particles while the HERWIG event generator is used for the RS graviton model. As both generators are LO event generators, the resulting cross-section predictions are multiplied by a k-factor of 1.3 to account for NLO corrections, as discussed in section 1.1.3.

\section{$Z^{\prime}$ limits}

The $Z^{\prime}$ model lines are calculated using the PYTHIA event generator with the $Z^{\prime}$ bosons only being allowed to decay to Standard Model particles. Additionally, the masses of $Z^{\prime}$ bosons are required to be within $10 \%$ of their on-shell mass. The couplings for the $E_{6} Z^{\prime}$ bosons can be found in table 1.4, while the SM-like $Z^{\prime}$ has the same couplings as the Standard Model $Z^{0}$. The neutral gauge bosons of the Standard Model are assumed not to mix with the $Z^{\prime}$ boson or otherwise interfere with it. Figure 7.9 shows the spin-1 $\sigma$.Br limits with the $Z^{\prime}$ model lines overlaid. The resulting $95 \%$ confidence level exclusion limits for the $Z^{\prime}$ models are summarised in table 7.4

\section{Previous Experimental Limits on $Z^{\prime}$ Bosons}

$Z^{\prime}$ bosons have been searched for by both CDF [10, 27, 61, 62] and D $\varnothing$ [63, 64] in the di-lepton and di-jet channels. The most stringent results come from the $e^{+} e^{-}$ and $\mu^{+} \mu^{-}$channels. The CDF $\tau^{+} \tau^{-}$search used a data sample corresponding to a luminosity of $200 \mathrm{pb}^{-1}$ and only manged to exclude a SM-like $Z^{\prime}$ boson with a mass below $399 \mathrm{GeV} / c^{2}\left[61\right.$. With the same dataset, the $e^{+} e^{-}$and $\mu^{+} \mu^{-}$channels individually excluded a SM-like $Z^{\prime}$ boson with a mass less than about $750 \mathrm{GeV} / \mathrm{c}^{2}$. 


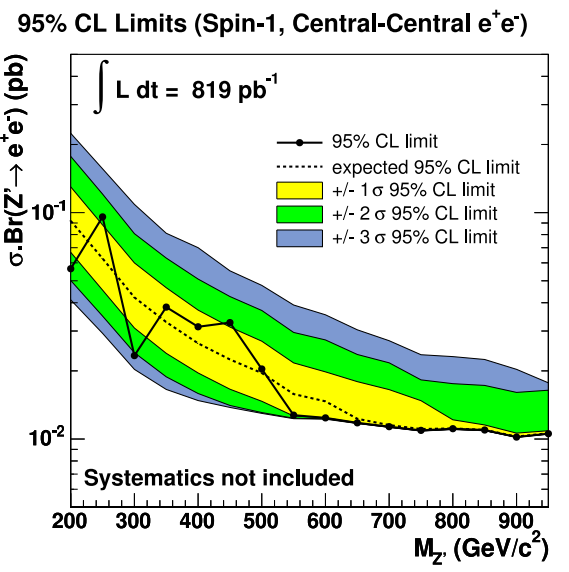

95\% CL Limits (Spin-1, Central-Central $\mathrm{e}^{+} \mathrm{e}^{-}$)

95\% CL Limits (Spin-1, Central-Plug $\mathrm{e}^{+} \mathrm{e}^{-}$)
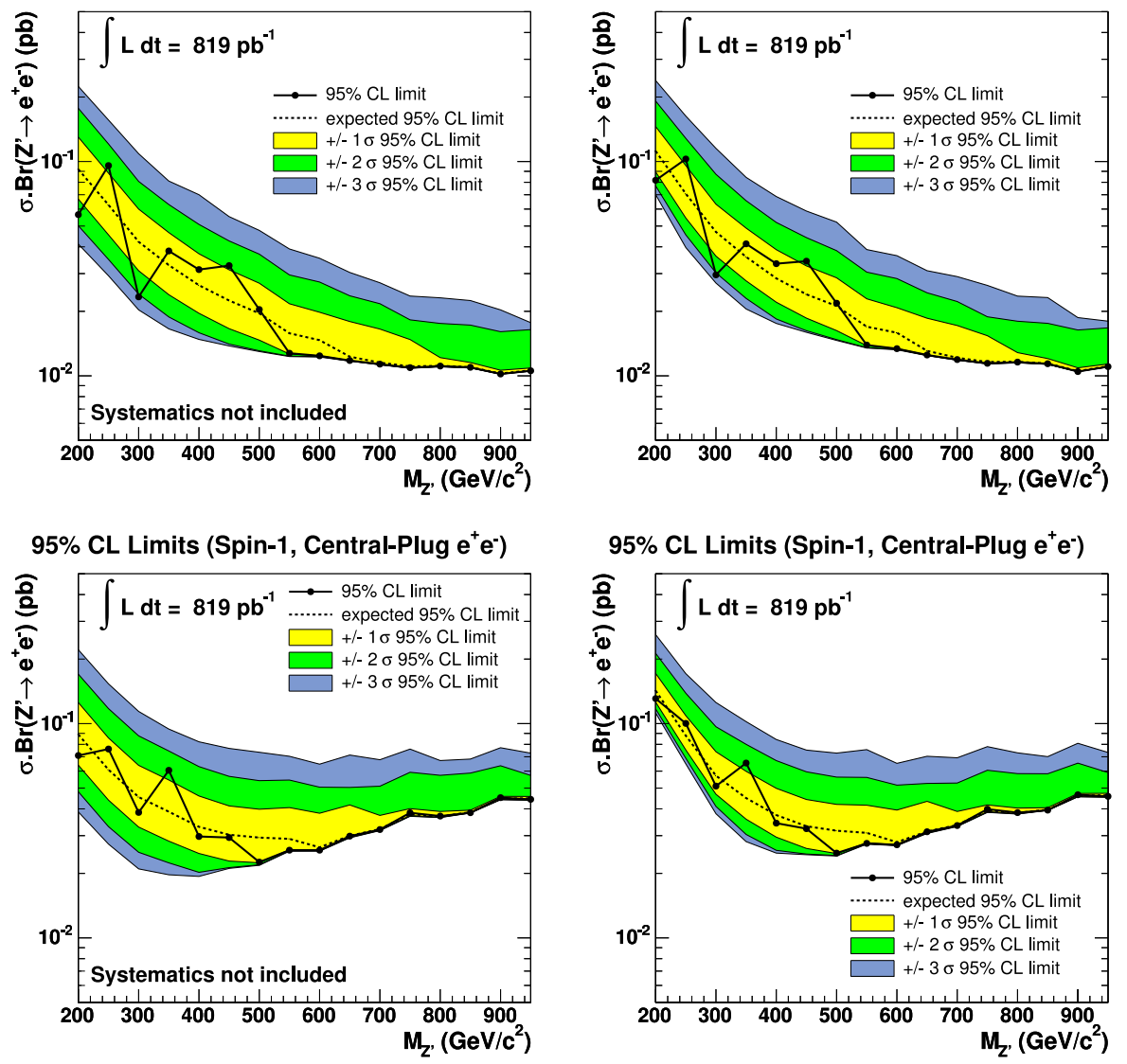

95\% CL Limits (Spin-1, Central-Plug $\mathrm{e}^{+} \mathrm{e}^{-}$)
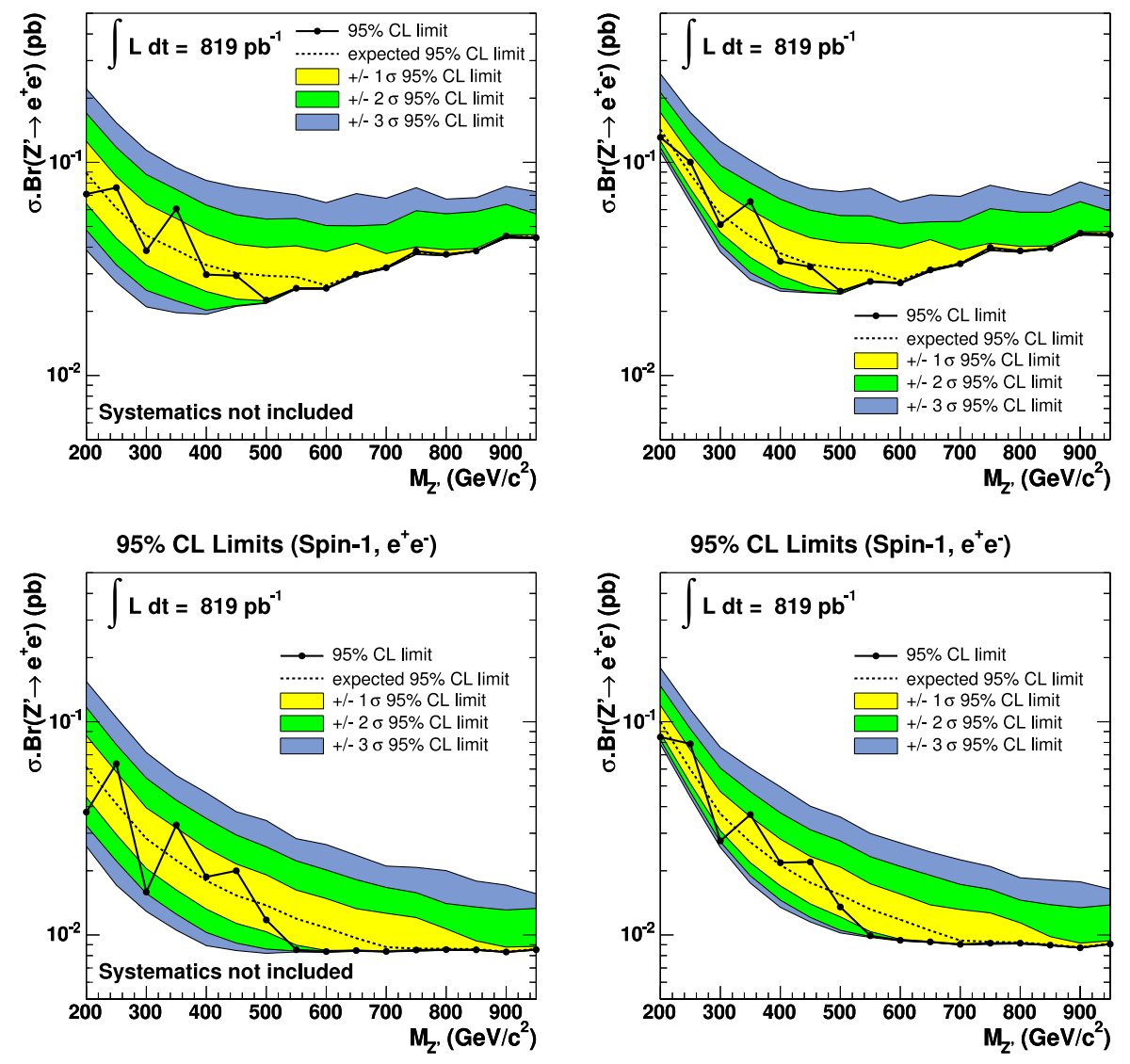

Figure 7.7: The 95\% CL limits for data for a spin-1 particle together with the expected limits from pseudo-experiments. The left plot shows the limits in the absence of systematics uncertainties and the right plot shows the limit in the presence of systematic uncertainties. 


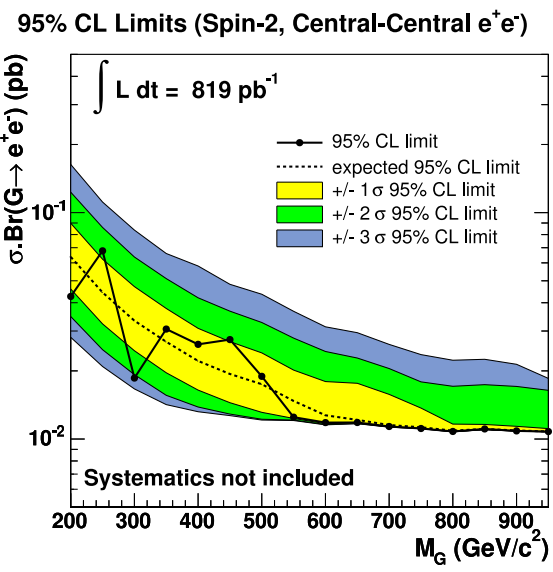

95\% CL Limits (Spin-2, Central-Central $\mathrm{e}^{+} \mathrm{e}^{-}$)

95\% CL Limits (Spin-2, Central-Plug e $\left.{ }^{+} e^{-}\right)$
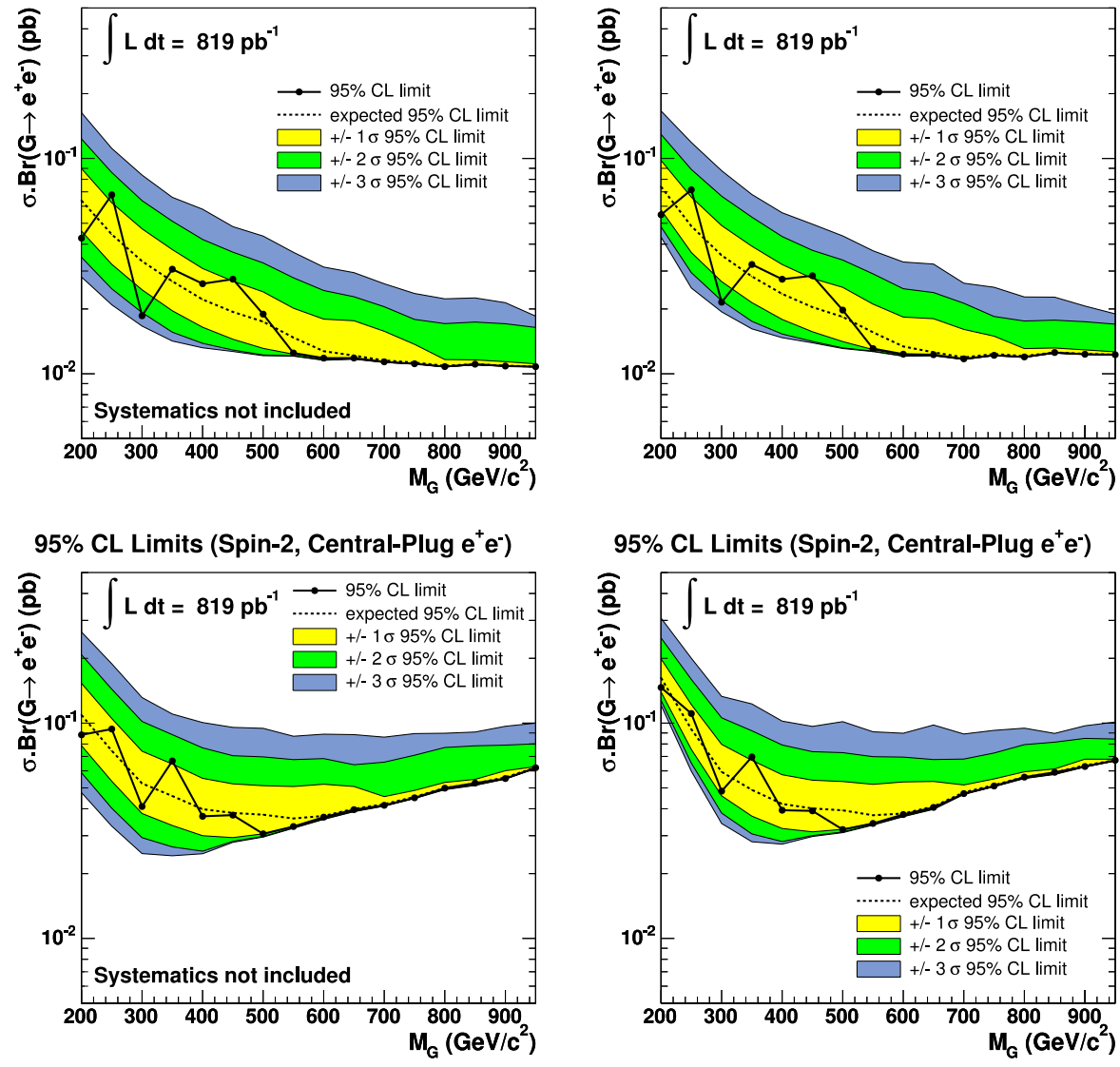

95\% CL Limits (Spin-2, Central-Plug $\mathrm{e}^{+} \mathrm{e}^{-}$)
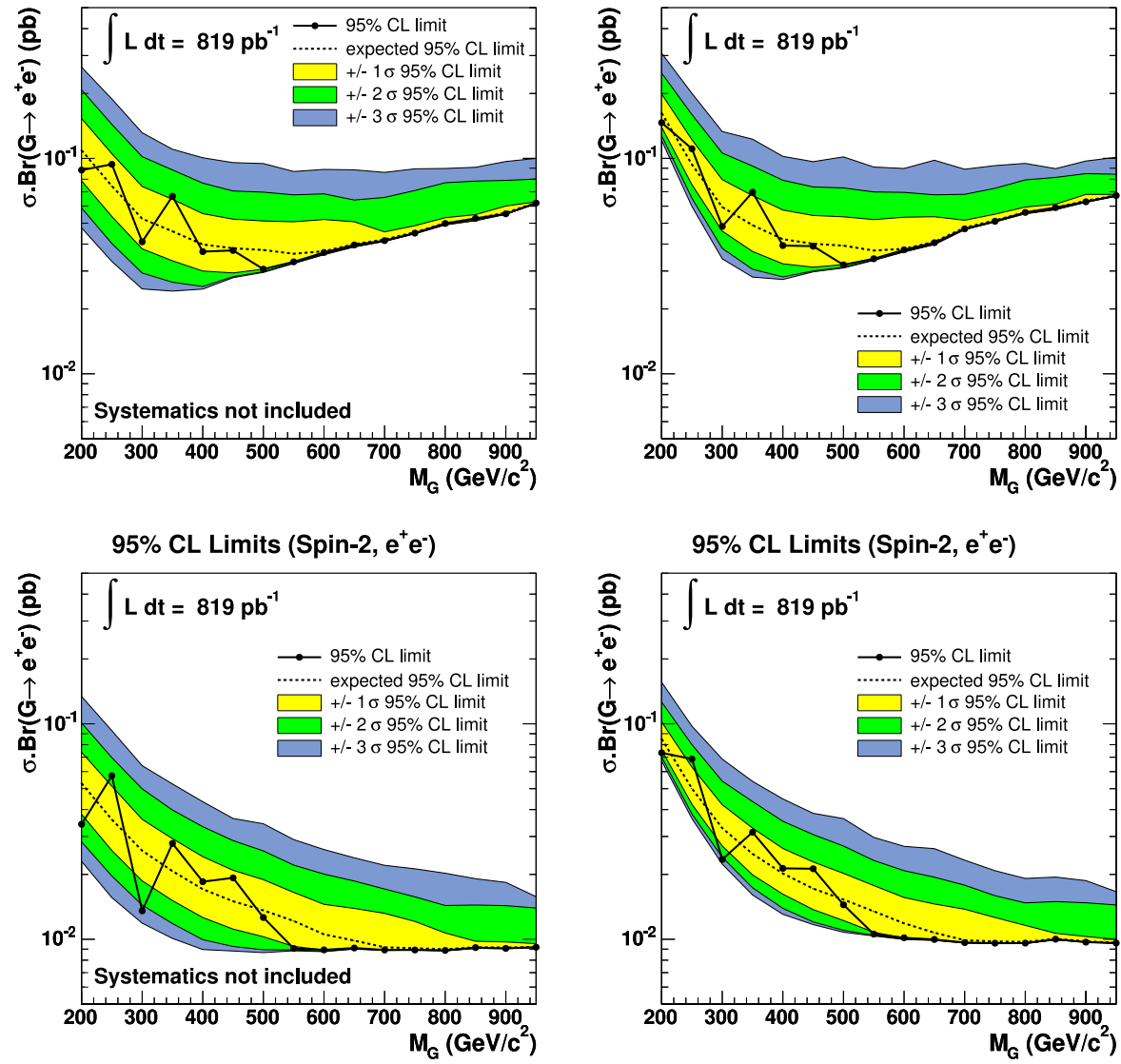

Figure 7.8: The 95\% CL limits for data for a spin-2 particle together with the expected limits from pseudo-experiments. The left plot shows the limits in the absence of systematics uncertainties and the right plot shows the limit in the presence of systematic uncertainties. 


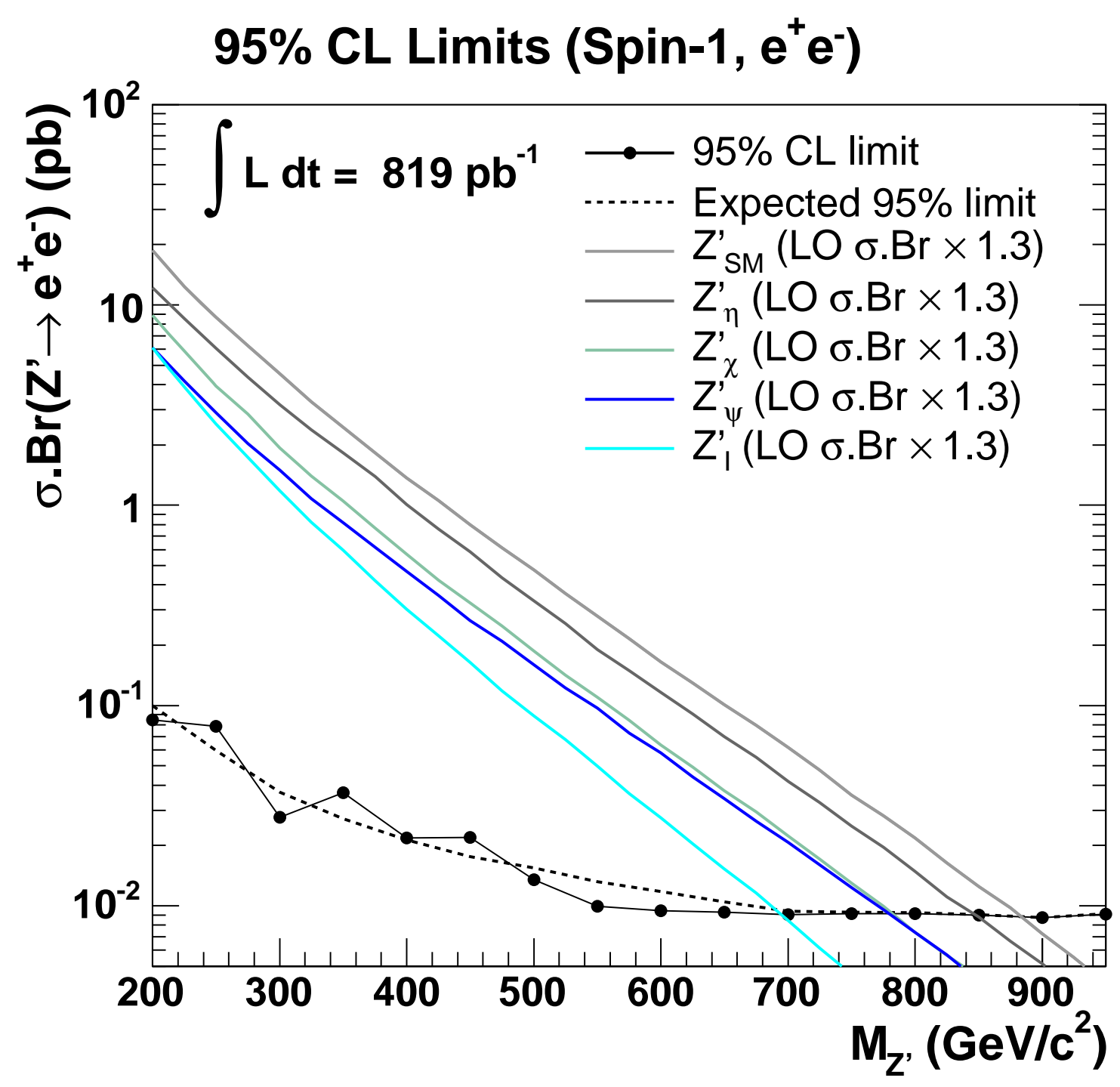

Figure 7.9: The observed and the expected 95\% confidence level limits on the $\sigma \cdot \operatorname{Br}\left(X \rightarrow e^{+} e^{-}\right)$of a spin-1 particle. The $\sigma \cdot \operatorname{Br}\left(Z^{\prime} \rightarrow e^{+} e^{-}\right)$as a function of mass for the $E_{6} Z^{\prime}$ bosons and the SM-like $Z^{\prime}$ boson are overlayed to obtain limits on the $Z^{\prime}$ boson masses. Starting at top, the model lines are for the SM-like $Z^{\prime}$, the $Z_{\eta}^{\prime}$, the $Z_{\chi}^{\prime}$, the $Z_{\psi}^{\prime}$ and the $Z_{I}^{\prime}$ bosons respectively. 


\begin{tabular}{|c|c|}
\hline$Z^{\prime}$ & Mass Limit $\left(\mathrm{GeV} / c^{2}\right)$ \\
\hline$Z_{S M}^{\prime}$ & 883 \\
$Z_{\eta}^{\prime}$ & 847 \\
$Z_{\chi}^{\prime}$ & 782 \\
$Z_{\psi}^{\prime}$ & 779 \\
$Z_{I}^{\prime}$ & 694 \\
\hline
\end{tabular}

Table 7.4: $Z^{\prime}$ mass limits for the $E_{6} Z^{\prime}$ bosons and the SM-like $Z^{\prime}$ at the $95 \%$ confidence level.

\begin{tabular}{|c|c|c|c|c|c|}
\hline \multirow{3}{*}{$Z^{\prime}$} & \multicolumn{5}{|c|}{ Mass Limit $\left(\mathrm{GeV} / c^{2}\right)$} \\
\hline & \multicolumn{3}{|c|}{$\mathrm{CDF}$} & $\mathrm{D} \varnothing($ Run I) & LEP \\
\hline & $\begin{array}{c}e^{+} e^{-} \\
\left(819 \mathrm{pb}^{-1}\right)\end{array}$ & $\begin{array}{c}e^{+} e^{-} \\
\left(448 \mathrm{pb}^{-1}\right)\end{array}$ & $\begin{array}{r}e^{+} e^{-}+\mu^{+} \mu \\
\left(200 \mathrm{pb}^{-1}\right)\end{array}$ & $\begin{array}{c}e^{+} e^{-} \\
\left(124.8 \mathrm{pb}^{-1}\right)\end{array}$ & indirect \\
\hline$Z_{S M}^{\prime}$ & 883 & 850 & 825 & 670 & 1500 \\
\hline$Z_{\eta}^{\prime}$ & 847 & 745 & 720 & - & 619 \\
\hline$Z_{\chi}^{\prime}$ & 782 & 740 & 690 & - & 781 \\
\hline$Z_{\psi}^{\imath}$ & 779 & 725 & 675 & - & 366 \\
\hline$Z_{I}^{\prime}$ & 694 & 650 & 615 & - & - \\
\hline
\end{tabular}

Table 7.5: $Z^{\prime}$ mass limits at the $95 \%$ confidence level for the $E_{6} Z^{\prime}$ bosons and the SM-like $Z^{\prime}$ obtained in this and previous analyses.

No di-jet search has been published in Run II by either CDF or DØ. In Run I, the CDF di-jet search failed to exclude the SM-like $Z^{\prime}$ boson at any mass [62] while the $\mathrm{D} \varnothing$ di-jet search excluded it in the mass range of $400-640 \mathrm{GeV} / c^{2}$ [64].

D $\varnothing$ has not yet chosen to publish a Run II $Z^{\prime}$ search and therefore their most recent published result is from Run I. However D $\varnothing$ have shown a preliminary Run II result at conferences. The most recent CDF search differed from previous analyses and this analyses as it used the measured forward-backward asymmetry of the $e^{+} e^{-}$events in addition to the mass spectrum to set more stringent limits [27].

The LEP experiments also set indirect limits on various $Z^{\prime}$ models [4]. The strength of the LEP limits is strongly dependent on the couplings of the $Z^{\prime}$ to Standard Model particles and therefore varies considerably for different models. The previous mass limits for the four $E_{6} Z^{\prime}$ s and the SM-like $Z^{\prime}$ are shown in table 7.5 .

\section{Technicolour Limits}

The Technicolour model line is calculated using the PYTHIA event generator, with all parameters set to the default values listed in section 1.2.2. Additionally, the masses of $\omega_{T C} / \rho_{T C}$ mesons are required to be within $10 \%$ of their on-shell mass. Figure 7.10 shows the spin-1 $\sigma$.Br limits with the technicolour model line overlaid. This gives an 


\begin{tabular}{|c|c|c|c|}
\hline Experiment & Channel & Luminosity & Mass Limit $\left(\mathrm{GeV} / c^{2}\right)$ \\
\hline \hline CDF & $\omega_{T C} / \rho_{T C} \rightarrow e^{+} e^{-}$ & $819 \mathrm{pb}^{-1}$ & 338 \\
\hline DØ Run I $)$ & $\omega_{T C} / \rho_{T C} \rightarrow e^{+} e^{-}$ & $124.8 \mathrm{pb}^{-1}$ & 203 \\
\hline DELPHI & $\begin{array}{c}\rho_{T C}(\gamma) \rightarrow W W, \gamma \pi_{T C}, \text { hadrons } \\
\rho_{T C}^{*} \rightarrow \pi_{T C} W_{L}, \pi_{T C} \pi_{T C}\end{array}$ & $452 \mathrm{pb}^{-1}$ & $90<M_{\rho_{T C}}<206.7$ \\
\hline
\end{tabular}

Table 7.6: The $95 \%$ confidence level limit on the $\omega_{T C} / \rho_{T C}$ mass, together with previous experimental limits.

exclusion limit on the vector technimeson mass of $338 \mathrm{GeV} / c^{2}$ at the $95 \%$ confidence level.

\section{Previous Experiment Limits on Technicolour}

Technicolour has been searched for in the $\omega_{T C} / \rho_{T C} \rightarrow l^{+} l^{-}$channel in previous CDF [10] and D 6 [63] analyses. Like for the $Z^{\prime}$ searches, D $\varnothing$ has chosen not to publish any Technicolour limits in Run II but has shown preliminary results at conferences. In Run I, DØ excludes a vector technimeson mass of below $203 \mathrm{GeV} / c^{2}$ for $M_{T}>200 \mathrm{GeV} / c^{2}$ at the $95 \%$ confidence level. The previous CDF result did not have sufficient sensitivity to exclude the vector technimesons in any mass range for $M_{T}=200 \mathrm{GeV} / c^{2}$. The DELPHI collaboration has also searched for Technicolour in the $e^{+} e^{-} \rightarrow \rho_{T C}(\gamma)$ channel with the $\rho_{T C}$ decaying to hadrons or longitudinally polarised $W$ bosons and the $e^{+} e^{-} \rightarrow \pi_{T C} \pi_{T C} ; \pi_{T C} W_{L}$ channels [65]. The DELPHI analysis excludes the $\rho_{T C}$ in a mass range of $90<M_{\rho_{T C}}<206.7 \mathrm{GeV} / c^{2}$ at the $95 \%$ confidence level and this result is independent of all other model parameters. Table 7.6 shows the $\omega_{T C} / \rho_{T C}$ mass limit obtained in this analysis with the previous experimental results.

\section{Randall-Sundrum Graviton Limits}

The Randall-Sundrum graviton model line is calculated using the HERWIG event generator and the graviton mass is constrained to be within $10 \%$ of the on-shell mass. Figure 7.11 shows the spin-2 $\sigma$. Br limits with the RS graviton model lines for various values of $k / \bar{M}_{p l}$ overlayed. The $95 \%$ confidence level limits on the graviton mass for various values of $k / \bar{M}_{p l}$ are summarised in table 7.7 .

\section{Previous Experimental Limits on RS Gravitons}

The Randall-Sundrum graviton has been searched for previously in the di-lepton channel at CDF [10] and the di-lepton and di-photon channel at D $\varnothing$ [66]. The results are summarised in table [7.8, together with combined di-electron and di-photon limits discussed in section 7.2.5. These were the first direct searches for the RS graviton although precision electroweak data has been used to constrain the model [25]. The precision electroweak constraints are complementary to the direct limits as they give 


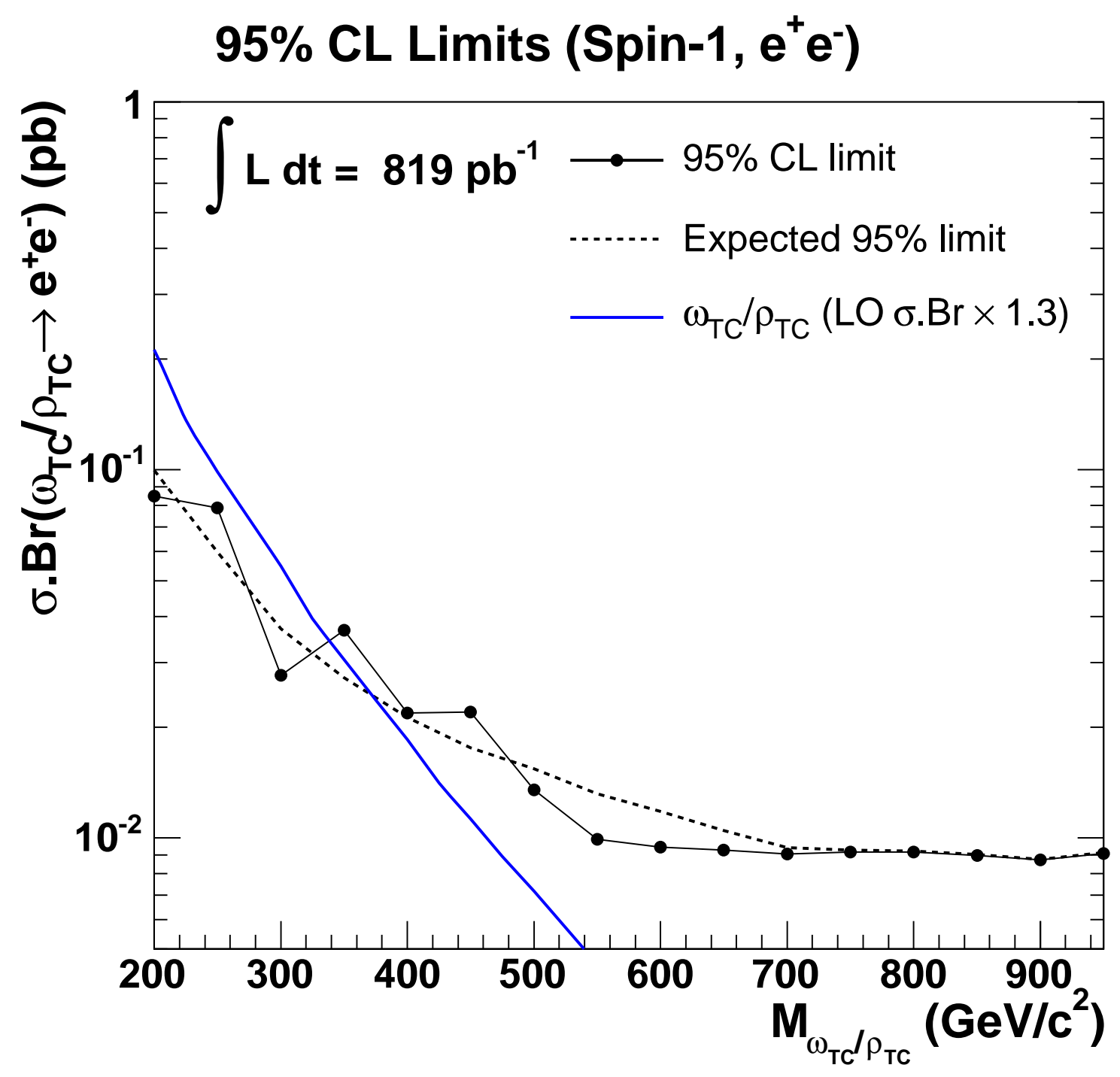

Figure 7.10: The observed and the expected 95\% confidence level limits on the $\sigma \cdot \operatorname{Br}\left(X \rightarrow e^{+} e^{-}\right)$of a spin-1 particle. The $\sigma \cdot \operatorname{Br}\left(\omega_{T C} / \rho_{T C} \rightarrow e^{+} e^{-}\right)$of a $\omega_{T C} / \rho_{T C}$ technimeson as a function of mass are overlayed to obtain limits on the vector technimeson mass. 


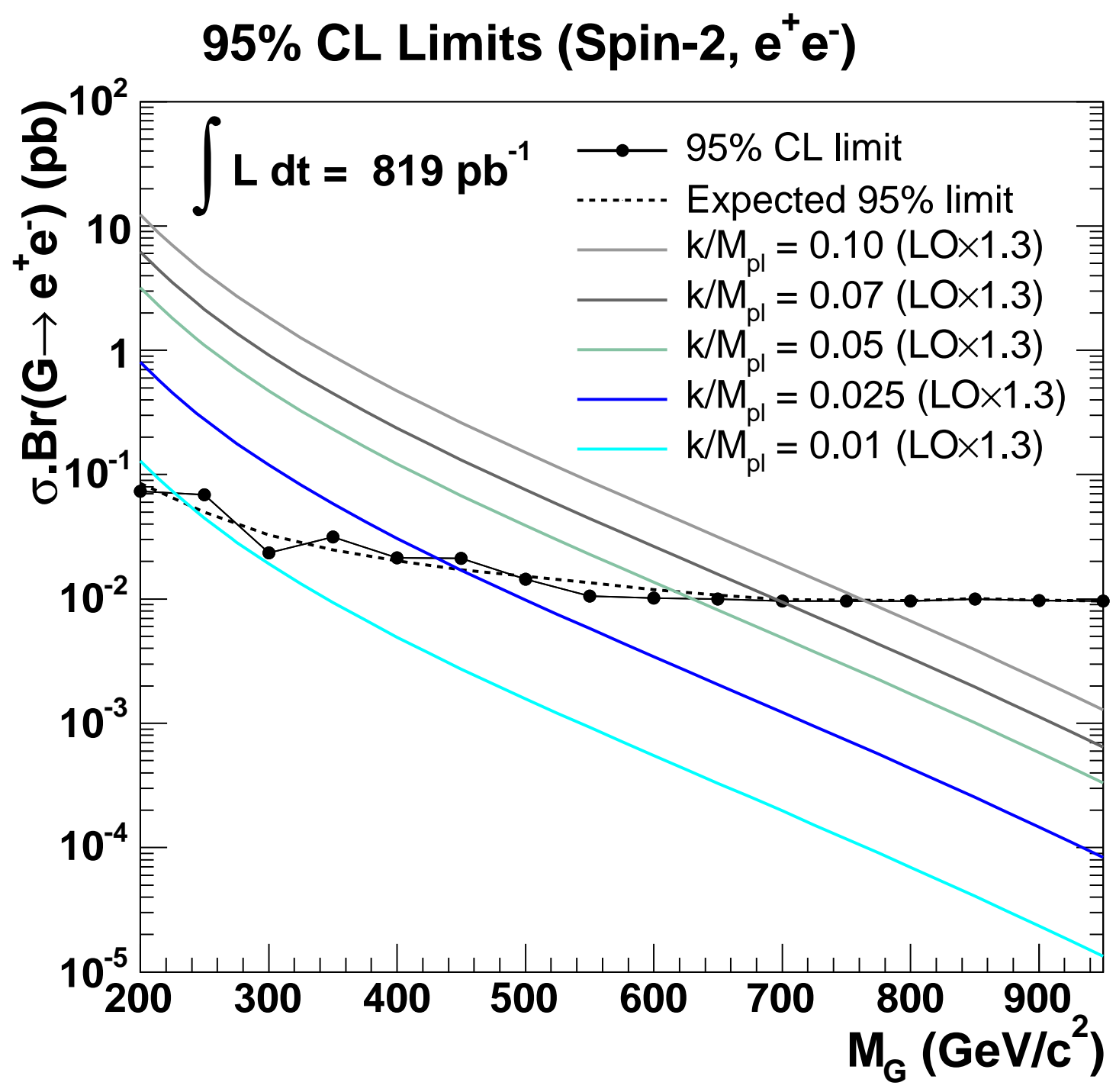

Figure 7.11: The observed and the expected 95\% confidence level limits on the $\sigma \cdot \operatorname{Br}\left(X \rightarrow e^{+} e^{-}\right)$of a spin-2 particle. The $\sigma \cdot \operatorname{Br}\left(G_{R S} \rightarrow e^{+} e^{-}\right)$of a RS graviton as a function of mass for various values of $k / \bar{M}_{p l}$ are overlayed to obtain mass and $k / \bar{M}_{p l}$ limits for the RS graviton. Starting at the top, the model lines are for $k / \bar{M}_{p l}$ values of $0.1,0.07,0.05,0.025$ and 0.01 respectively. 


\begin{tabular}{|c|c|}
\hline$k / M_{p l}$ & Mass Limit $\left(\mathrm{GeV} / c^{2}\right)$ \\
\hline 0.1 & 764 \\
0.07 & 700 \\
0.05 & 629 \\
0.025 & 431 \\
0.01 & 230 \\
\hline
\end{tabular}

Table 7.7: Randall-Sundrum graviton mass limits for various values of $k / \bar{M}_{p l}$ at the $95 \%$ confidence level.

\begin{tabular}{|c|c|c|c|c|}
\hline \multirow{3}{*}{$k / \bar{M}_{p l}$} & \multicolumn{4}{|c|}{ Mass Limit $\left(\mathrm{GeV} / c^{2}\right)$} \\
\cline { 2 - 5 } & $e^{+} e^{-}$ & $\begin{array}{c}e^{+} e^{-}+\gamma \gamma \\
\left(0.8-1.2 \mathrm{fb}^{-1}\right)\end{array}$ & $\begin{array}{c}e^{+} e^{-}+\mu^{+} \mu^{-} \\
\left(200 \mathrm{pb}^{-1}\right)\end{array}$ & $\begin{array}{c}e^{+} e^{-}+\gamma \gamma \\
\left(275 \mathrm{pb}^{-1}\right)\end{array}$ \\
\cline { 2 - 5 } & $\left(819 \mathrm{pb}^{-1}\right)$ & 710 & 785 \\
0.1 & 764 & 874 & 170 & 250 \\
0.01 & 230 & 244 & 170 \\
\hline
\end{tabular}

Table 7.8: Randall-Sundrum graviton mass limits at the $95 \%$ confidence level for various values of $k / \bar{M}_{p l}$ together with previous experimental results.

a maximum on $k / \bar{M}_{p l}$ at a given mass while the direct limits give a minimum on $k / \bar{M}_{p l}$ for a given mass. The precision electroweak constraints exclude $k / \bar{M}_{p l} \leq 0.1$ for $M_{G}=200 \mathrm{GeV} / c^{2}$ which falls to $k / \bar{M}_{p l} \leq 0.025$ at $M_{G} \approx 900 \mathrm{GeV} / c^{2}$ [25]. Using both the direct and indirect limits, there is complete exclusion of all values of $k / \bar{M}_{p l}$ up to a graviton mass of $\sim 600 \mathrm{GeV} / c^{2}$.

\subsubsection{Combination with the Di-Photon Channel}

The Randall-Sundrum graviton couples to all Standard Model particles and the relative branching ratios can be found in 25 . At CDF, the RS graviton is searched for in the di-photon, $Z^{0} Z^{0}$, di-lepton and di-jet channels. Currently only the di-photon channel is available for combination; the other channels are either not ready to be combined or have insufficient sensitivity to make the combination worthwhile.

The di-photon channel is an ideal channel to combine with the di-electrons due to the similarity of the two objects. Both appear experimentally as deposits of energy in the electromagnetic calorimeter, with the main distinguishing feature being the presence of a track in the COT for electrons. Hence the systematics uncertainties are similar and the correlations are simple to evaluate. The RS graviton has twice the branching ratio to photons as it does to electrons [25] which makes the combination simple. In addition, no events overlap in the di-photon and di-electron channels as the di-electron analysis requires that central electrons have an associated track and the di-photon analysis requires that central photons have no associated track. 
The di-photon analysis is performed with a data sample corresponding to $1155 \mathrm{pb}^{-1}$ and is fully documented in [45. The di-photon analysis requires one photon in the central calorimeter and a second photon in either the central or plug calorimeters. The photons are required to have an $E_{T} \geq 15 \mathrm{GeV}$ and be in the region $|\eta| \leq 1.1$ or $1.2 \leq|\eta| \leq 2.8$ depending on whether the photon is incident in the CEM or PEM. The photons are also required to be well isolated, have relatively little hadron energy, no associated track and also have a electromagnetic shower profile which is consistent with that of a photon 45 .

Photon reconstruction is identical to electron reconstruction at CDF, with the exception that central photons obtain angular information from the CES rather than from the highest $p_{T}$ track associated with the cluster. The photon energy is defined exactly the same way as the electron energy and the same calibrations are applied to data and Monte Carlo simulated events as are applied to electrons. All Monte Carlo samples used in the di-photon analysis are generated using the CTEQ5L PDFs, the same as for the di-electron analysis. The di-photon analysis uses the HERWIG event generator to produce the $G_{R S} \rightarrow \gamma \gamma$ signal samples and the graviton mass is required to be within $10 \%$ of the on-shell mass. The value of $k / \bar{M}_{p l}$ used in the signal samples is 0.1 and is the same value used to generate the corresponding di-electron samples. The background estimate for the di-photon channel is obtained by a fit to data and Monte Carlo simulated di-photon events generated by the DIPHOX NLO matrix element generator [67]. The uncertainty on the fit is taken as the systematic uncertainity on the background estimate, and this is approximately $50 \%-60 \%$ in the central-central channel and $40 \%-140 \%$ in the central-plug channel, with the percentage uncertainty increasing as the mass increases. Above approximately $M_{\gamma \gamma}=500 \mathrm{GeV} / c^{2}$, the predicted background is close to zero.

In the limit calculation, the central-central and central-plug di-photon channels are treated identically to the two di-electron channels. The combination procedure between the di-electron and di-photon channels is thus identical in principle to the combination of the di-electron central-central and central-plug channels, and is achieved by multiplying the likelihoods together. The treatment of systematic uncertainties is also identical to the treatment of systematic uncertainties for the central-central and central-plug channel combination. The systematic uncertainties affecting each channel are summarised in table 7.9. The majority of the sources of systematic uncertainty in the di-photon channel are exactly the same as those in the di-electron channel. These are the uncertainties on the luminosity, the CTEQ5L PDFs, the ISR and the Monte Carlo simulation energy scale and resolution. The di-photon channel is also significantly affected by a $10 \%$ uncertainty on the photon coversion probability. The uncertainties on the electron and photon identification efficiencies are assumed to be $100 \%$ correlated in the individual calorimeter regions to simplify the combination. This is true to a good approximation as the selection cuts are similar and any disagreement in the Monte Carlo simulation of the identification variables for EM clusters will effect both channels almost equally. The uncertainties on the background estimates are all taken to be uncorrelated with each other, as the backgrounds arise from different 
sources and are evaluated using different methods.

The two analyses evaluate the effects of the PDF uncertainties differently. The di-electron analysis keeps track of all the 20 different PDF eigenvector uncertainties separately and evaluates the effect on the background estimation as well as the signal acceptance for each mass point. The di-photon analysis sums the PDF eigenvector uncertainties for each mass point and then takes the largest uncertainty on the acceptance as the uncertainty for all the mass points. This uncertainty is found to be $4 \%$ for both central-central and central-plug channels. When using this simpler method in the di-electron analysis, uncertainties of $3.9 \%$ and $5.2 \%$ are obtained on the acceptance in the central-central and the central-plug channels respectively due to the PDF uncertainties. Figure 7.12 shows the resulting di-electron spin-2 limits using this simpler method for evaluating the effect of the PDF uncertainties and those obtained using the normal method. As can be seen from figure 7.12, the difference is small at high mass although at $M_{G}=200 \mathrm{GeV} / c^{2}$, the simplified method significantly underestimates the effect of the PDF uncertainties. However in this region, the large systematic uncertainty of the di-photon background dominates, making it acceptable to use the simplified approach for the di-photon and the combined limits. The di-electron analysis only uses the simplified treatment of the PDF uncertainties when combining with the di-photon channel and uses the full treatment otherwise.

The Monte Carlo simulation energy scale and resolution are also evaluated differently. In the di-electron analysis, these uncertainties principally affect the background shape obtained from the Monte Carlo simulation. The di-photon analysis uses a fit to data and Monte Carlo simulated events to obtain the background. As the fit depends significantly on the data, the Monte Carlo energy scale and resolution effects become less important and, given the already large uncertainties on the fit, they can be safely ignored.

The result of the combination of the di-electron and di-photon channels is shown in figure 7.13 as a direct limit on $\sigma \cdot \operatorname{Br}\left(G_{R S} \rightarrow l^{+} l^{-}\right)$and also as a $k / \bar{M}_{p l}$ vs $M_{G}$ exclusion plane. The mass limits for various values of $k / \bar{M}_{p l}$ are also summarised in table 7.10 . As can be seen both from figure 7.13 and table 7.10, the combined limits significantly improve upon the limits from the individual channels. These are the most stringent set of direct limits on RS graviton production to date. 


\begin{tabular}{|c|c|c|c|c|c|}
\hline & \multirow{3}{*}{ Quantity } & \multicolumn{3}{|c|}{ Channels Effected } \\
\cline { 3 - 6 } & Uncertainty & $e^{+} e^{-}$ & \multicolumn{2}{|c|}{$\gamma \gamma$} \\
\cline { 3 - 6 } & & $\mathrm{CC}$ & $\mathrm{CP}$ & $\mathrm{CC}$ & $\mathrm{CP}$ \\
\hline Luminosity & $6 \%$ & $\sqrt{ }$ & $\sqrt{ }$ & $\sqrt{ }$ & $\sqrt{ }$ \\
Photon Conversion & $10 \%$ & $\sqrt{ }$ & $\sqrt{ }$ & $\sqrt{ }$ & $\sqrt{ }$ \\
High Mass Eff. (CEM) & $2 \%$ & $\sqrt{ }$ & $\sqrt{ }$ & $\sqrt{ }$ & $\sqrt{ }$ \\
High Mass Eff. (PEM) & $2 \%$ & $\mathrm{X}$ & $\sqrt{ }$ & $\mathrm{X}$ & $\sqrt{ }$ \\
\hline PDF Uncertainties & see text & $\sqrt{ }$ & $\sqrt{ }$ & $\sqrt{ }$ & $\sqrt{ }$ \\
Initial State Radiation & $4 \%$ on acceptance & $\sqrt{ }$ & $\sqrt{ }$ & $\sqrt{ }$ & $\sqrt{ }$ \\
MC Energy Scale (CEM) & $1 \%$ & $\sqrt{ }$ & $\sqrt{ }$ & $\sqrt{ }$ & $\sqrt{ }$ \\
MC Energy Scale (PEM) & $1 \%$ & $\mathrm{X}$ & $\sqrt{ }$ & $\mathrm{X}$ & $\sqrt{ }$ \\
MC Energy Res. (CEM) & $0.7 \%$ & $\sqrt{ }$ & $\sqrt{ }$ & $\sqrt{ }$ & $\sqrt{ }$ \\
MC Energy Res. (PEM) & $0.5 \%$ & $\mathrm{X}$ & $\sqrt{ }$ & $\mathrm{X}$ & $\sqrt{ }$ \\
\hline$e^{+} e^{-}$Jet Bkg. Norm. (CC) & $85.8 \%$ & $\sqrt{ }$ & $\mathrm{X}$ & $\mathrm{X}$ & $\mathrm{X}$ \\
$e^{+} e^{-}$Jet Bkg. Norm. (CP) & $32.0 \%$ & $\mathrm{X}$ & $\sqrt{ }$ & $\mathrm{X}$ & $\mathrm{X}$ \\
$e^{+} e^{-}$Jet Bkg. Shape (CC) & see text & $\sqrt{ }$ & $\mathrm{X}$ & $\mathrm{X}$ & $\mathrm{X}$ \\
$e^{+} e^{-}$Jet Bkg. Shape (CP) & see text & $\mathrm{X}$ & $\sqrt{ }$ & $\mathrm{X}$ & $\mathrm{X}$ \\
$Z^{0} / \gamma^{*} \rightarrow e^{+} e^{-}$MC Norm. & $3.8 \%$ & $\sqrt{ }$ & $\sqrt{ }$ & $\mathrm{X}$ & $\mathrm{X}$ \\
$\gamma \gamma$ Bkg (CC) & see text & $\mathrm{X}$ & $\mathrm{X}$ & $\sqrt{ }$ & $\mathrm{X}$ \\
$\gamma \gamma$ Bkg (CP) & see text & $\mathrm{X}$ & $\mathrm{X}$ & $\mathrm{X}$ & $\sqrt{ }$ \\
\hline \hline
\end{tabular}

Table 7.9: Summary of systematic uncertainties affecting the $e^{+} e^{-}$and $\gamma \gamma$ channels with a $\sqrt{ }$ indicating the channel is affected by the uncertainty and $\mathrm{X}$ indicating it is not. Uncertainties that are listed as "see text" are uncertainties which can not easily be expressed as a single number. Note that while the photon conversion uncertainty affects the di-electron channel, the effect is negligible. In addition, while the MC Energy scale and resolution uncertainties do affect the di-photon channel, the effects are also negligible.

\begin{tabular}{|c|c|c|c|}
\hline \multirow{2}{*}{$k / \bar{M}_{p l}$} & \multicolumn{3}{|c|}{ Mass Limit $\left(\mathrm{GeV} / c^{2}\right)$} \\
\cline { 2 - 4 } & $e^{+} e^{-}$ & $\gamma \gamma$ & $e^{+} e^{-}+\gamma \gamma$ \\
\hline 0.1 & 764 & 850 & 874 \\
0.07 & 700 & 782 & 810 \\
0.05 & 629 & 695 & 750 \\
0.025 & 431 & 496 & 568 \\
0.01 & 230 & 230 & 244 \\
\hline
\end{tabular}

Table 7.10: Randall-Sundrum graviton mass limits for various values of $k / \bar{M}_{p l}$ for the di-photon and di-electron channels seperately and combined at the $95 \%$ confidence level. 


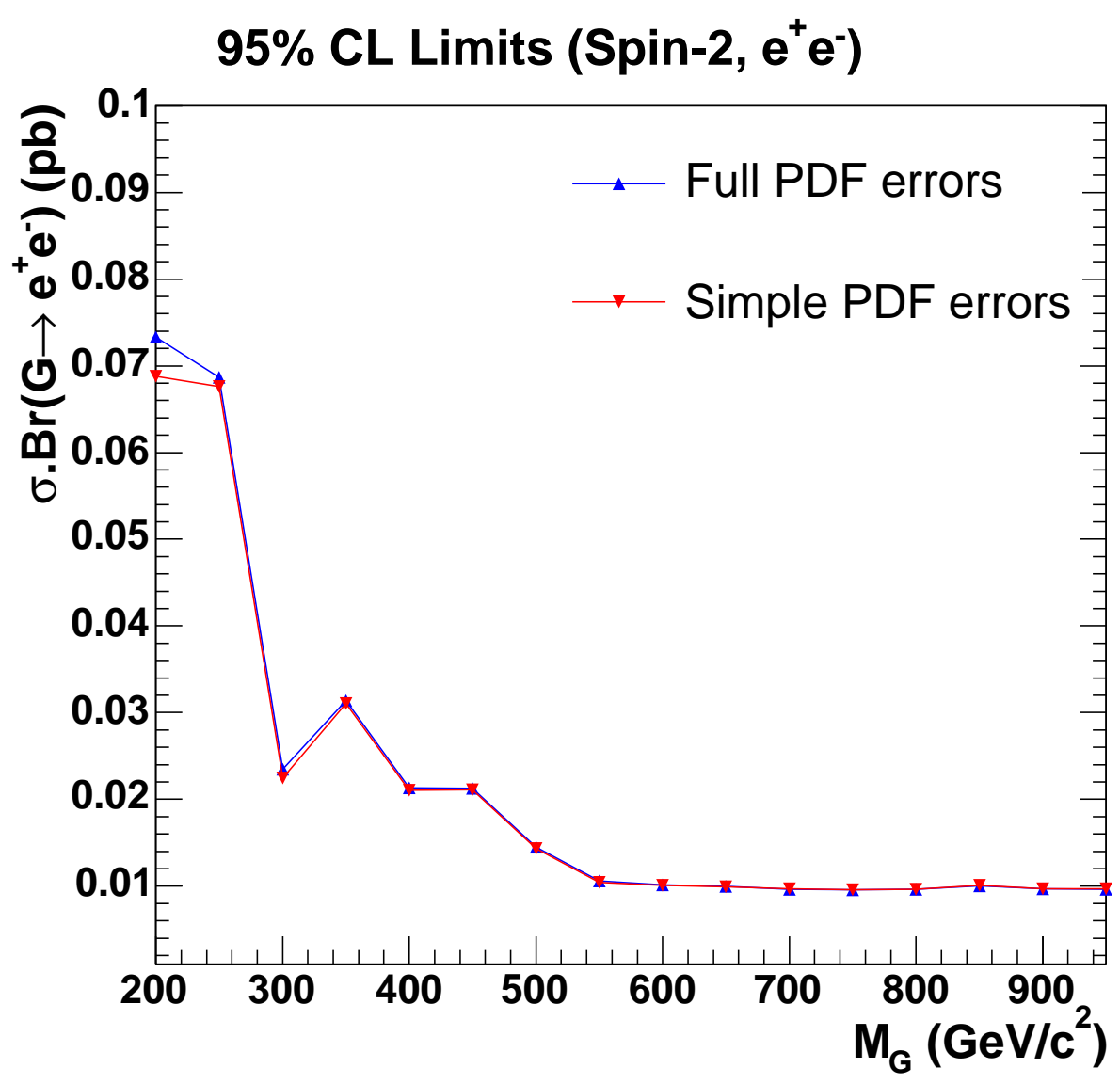

Figure 7.12: A comparison of the spin-2 limits obtained for a full and for a simple treatment of PDF uncertainties. The only significant difference is at low mass where a large di-photon background uncertainty will dominate the combined limits. 


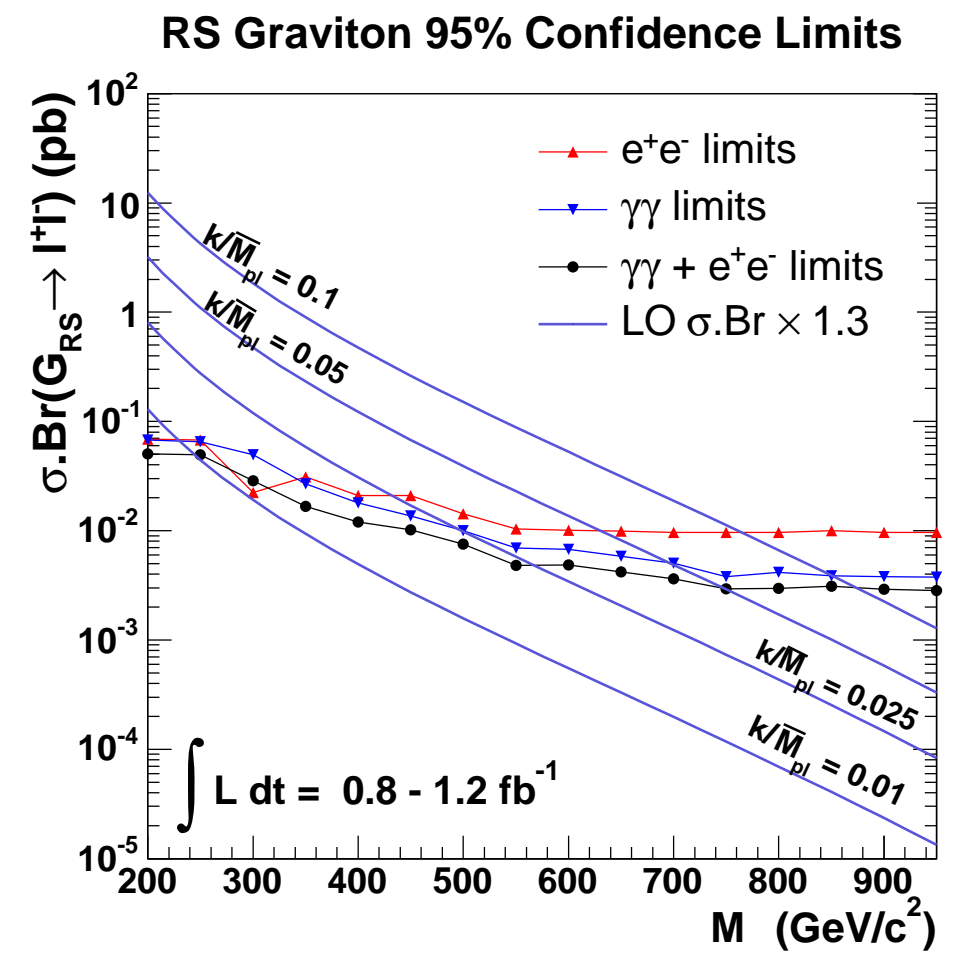

\section{$\mathrm{k} / \overline{\mathrm{M}}_{\mathrm{pl}}$ vs RS Graviton Mass Exclusion Plot}

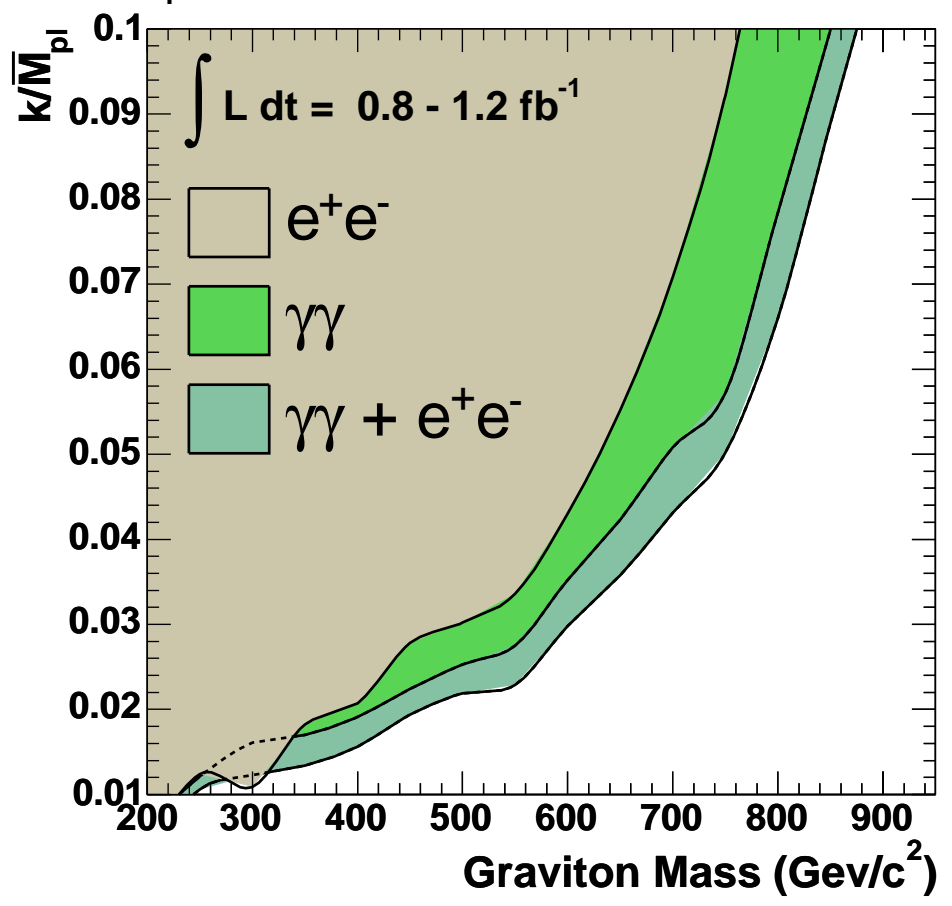

Figure 7.13: The top plot shows the observed 95\% confidence level limits on the $\sigma \cdot \operatorname{Br}\left(G_{R S} \rightarrow l^{+} l^{-}\right)$of the RS graviton from the di-electron and di-photon channels and their combination. The bottom plot shows the $\sigma \cdot \operatorname{Br}\left(G_{R S} \rightarrow l^{+} l^{-}\right)$limits converted into a $k / \bar{M}_{p l}$ vs graviton mass exclusion plane. 


\section{Chapter 8}

\section{Conclusions}

A narrow resonance decaying to a di-electron pair was searched for in the invariant mass range of $150 \leq M_{e e} \leq 950 \mathrm{GeV} / c^{2}$ using $819 \mathrm{pb}^{-1}$ of Tevatron $p \bar{p}$ collision data. The electrons are both required to have $E_{T} \geq 25 \mathrm{GeV}$ and one electron is required to be in the range $|\eta| \leq 1.1$ and the other in the range $|\eta| \leq 1.1$ or $1.2 \leq|\eta| \leq 3.0$. No significant evidence is found for such a resonance. Limits are set on the production at the Tevatron of spin-1 or spin-2 particles decaying to di-electron pairs in a mass range of $200-950 \mathrm{GeV} / c^{2}$. At masses greater than $550 \mathrm{GeV}$, spin-1 and spin-2 bosons with a $\sigma \cdot \operatorname{Br}\left(p \bar{p} \rightarrow X \rightarrow e^{+} e^{-}\right)>0.01 \mathrm{pb}$ are excluded at the $95 \%$ confidence level. The $E_{6} Z^{\prime}$ bosons, the $Z_{I}^{\prime}$, the $Z_{\psi}^{\prime}$, the $Z_{\chi}^{\prime}$ and the $Z_{\eta}^{\prime}$ bosons, are excluded at the $95 \%$ confidence level with masses below $694,779,782$ and $847 \mathrm{GeV} / c^{2}$ respectively. The SM-like $Z^{\prime}$ is excluded at the $95 \%$ confidence level for masses below $883 \mathrm{GeV} / c^{2}$. The techniomega and technirho are excluded at the $95 \%$ confidence level for masses below $338 \mathrm{GeV} / c^{2}$. The RS graviton is also excluded at the $95 \%$ confidence level for a graviton mass less than $764(230) \mathrm{GeV} / c^{2}$ for $k / \bar{M}_{p l}=0.1(0.01)$. When combined with the di-photon channel, the RS graviton exclusion limits improve to exclude a graviton with a mass less than $874(244) \mathrm{GeV} / c^{2}$ for $k / \bar{M}_{p l}=0.1(0.01)$ at the $95 \%$ confidence level. A comparison with previous results for the various models analysed is detailed in chapter 7 . In most cases the limits given here are the most stringent yet obtained and for the first time, the direct limits have surpased the indirect limits from electroweak fits for all canonical $E_{6} Z^{\prime}$ bosons.

\subsection{Future Prospects}

This analysis is not yet systematics limited and the sensitivity of the analysis will continue to improve with more data. The analysis can also be improved by increasing the acceptance by adding in plug-plug events or by adding in the angular information of the electrons which has been done in [27]. In this previous analysis, adding in the angular information improved the limit for the SM-like $Z^{\prime}$ by an amount that would having required $25 \%$ more data using the mass spectrum alone. The plug-plug channel has an acceptance of about $7 \%$ to $16 \%$, with the acceptance increasing as the mass 
increases. This would increase the sensitivity of the analysis in the high mass region. A combination with the di-muon channel would also lead to an improved analysis senstivity although at high mass, the di-muon mass resolution is poor at CDF.

The Tevatron has a mass reach of $\approx 1 \mathrm{TeV}$ and to probe past this region, the LHC will be required. The LHC is a proton-proton collider with $\sqrt{s}=14 \mathrm{TeV}$. Experiments at the LHC will have sensitivity to a new physics resonance with a mass of about $3.5-5 \mathrm{TeV}$ [25, 68] with approximately $100 \mathrm{fb}^{-1}$ of data. A search such as this is an ideal early search at the LHC as it has a straight forward final state and can be calibrated on the $Z^{0}$ resonance. However due to the increased search range, a large amount of data will be required to ensure that any excess is not simply a statistical fluctuation. 


\section{Appendix A}

\section{Electron Identification Cut Selection}

Two sets of electron selection cuts existed at CDF before this analysis was started. These were the standard CDF high- $p_{T}$ electron selection cuts and the cuts used by the previous iteration of this analysis, known as the 'old very high- $p_{T}$ cuts'. Both sets of cuts are shown in tables A.1 and A.2 for CEM electrons and PEM electrons respectively. A major problem with the standard cuts is that they have a low efficiency of $\sim 80 \%$ per electron. Since the background in high mass di-electron pairs is low, the analysis sensitivity would be improved by more efficient identification cuts. The old very high- $p_{T}$ cuts are looser for this reason, but both sets of cuts are optimised for the kinematic regime around the $Z^{0}$ pole. Therefore the selection cuts were re-developed to produce cuts optimised for very high energy electrons.

The cuts are re-optimised by considering their N-1 identification efficiency and N-1 background rejection as a function of electron $E_{T}$. The N-1 efficiency is defined in section 5.1 and the $\mathrm{N}-1$ background rejection is a similar concept. The N-1 background rejection of the $i^{\text {th }}$ cut is the fraction of 'fake' electrons which have passed all the other cuts but are rejected by applying the $i^{\text {th }}$ cut. The sample of fake electrons used to measure this is constructed by first taking the jet background sample obtained in section 6.2.2. The additional requirements that the fake electron is reasonably backto-back in $\phi$ with the partner jet $\left(135^{\circ} \leq \Delta \phi \leq 225^{\circ}\right)$ and that the missing $E_{T}$ in the event is low $\left(S_{M E T} \leq 2.5\right)$ are applied to further ensure the purity of the fake sample. To determine the efficiency at high $E_{T}\left(E_{T}>70 \mathrm{GeV}\right)$ Monte Carlo generated events are used, as in section 5.1. This is necessary as an electron sample, with a high enough purity to perform a reliable efficiency measurement, only exists in the data at the $Z^{0}$ pole which, due to kinematic constraints, gives poor statistics above $E_{T} \sim 70 \mathrm{GeV}$.

The goal is to choose the cuts so that their efficiency does not decrease as the electron $E_{T}$ increases and that their efficiency and background rejection versus $E_{T}$ is smooth and does not have any points where it discontinuously jumps or falls. In addition, the ability of a cut to maintain its background rejection power at high energies is also important. The cuts are loosely optimised following these guidelines, with 


\begin{tabular}{|c|c|c|}
\hline Variable & Standard Cuts & Old Very High- $p_{T}$ Cuts \\
\hline \hline Region & $=\mathrm{CEM}$ & $=\mathrm{CEM}$ \\
$E_{T}$ & $\geq 20 \mathrm{GeV}$ & $\geq 25 \mathrm{GeV}$ \\
Track $p_{T}$ & $\geq 10 \mathrm{GeV} / c$ & Fid $=1$ \\
Fiducial & Fid $=1$ & $\leq 60 \mathrm{~cm}$ \\
Track $\left|z_{0}\right|$ & $\leq 60 \mathrm{~cm}$ & $\leq 0.1$ \\
\hline Had $/ \mathrm{em}$ & $\leq 0.055+0.00045 \times E$ & $\leq 0.055+0.00045 \times E$ \\
Isolation $E_{T} / E_{T}$ & $\leq 0.1$ & $\leq 0.2$ \\
$L_{S h r}$ & $\leq 0.2$ & $\leq 5.0 \mathrm{~cm}$ \\
$E / p$ & $\leq 2$ unless $p_{T} \geq 50 \mathrm{GeV} / c$ & $\leq 4 \mathrm{unless} E_{T} \geq 100 \mathrm{GeV}$ \\
CES $\Delta Z$ & $\leq 3.0 \mathrm{~cm}$ & - \\
CES $\Delta X$ & $-3.0 \leq \Delta X \times q \leq 1.5 \mathrm{~cm}$ & - \\
CES $\chi_{\text {strip }}^{2}$ & $\leq 10$ & - \\
COT Ax. Segs. & $\geq 3$ & - \\
COT St. Segs. & $\geq 2$ & \\
Conversion & $\neq 1$ & \\
\hline
\end{tabular}

Table A.1: The standard CEM high- $p_{T}$ cuts used by CDF in a kinematic region around the $Z^{0}$ pole and the old very high- $p_{T}$ CEM cuts used by the previous high mass dielectron analysis. For the had/em cut, $E$ is in units of $\mathrm{GeV}$. For the $\Delta X$ cut, $q$ is the charge of the electron. The first section of the table lists the kinematic and geometric cuts and the second section lists the identification cuts.

attempts to be relatively conservative. Due to this philosophy, often the modified cuts are equal to the standard cuts at the $Z^{0}$ pole, and just evolve differently as a function of energy. More precise tuning of these cuts is not appropriate, due to the known limitations of the Monte Carlo simulation used to obtain the efficiency estimates. In addition the background estimates have large uncertainties which would further increase the uncertainties of any signal to background comparison for an individual cut.

The performance of the cuts resulting from this re-optimisation is compared with the performance of the old very high- $p_{T}$ cuts for masses above $200 \mathrm{GeV} / c^{2}$ in table A.3. The number of events above $M_{e e}=200 \mathrm{GeV} / c^{2}$ passing the old very high- $p_{T}$ cuts are obtained from [10] and are for a data sample of $200 \mathrm{pb}^{-1}$. The number of events above $M_{e e}=200 \mathrm{GeV} / c^{2}$ passing the new cuts are for a data sample of $819 \mathrm{pb}^{-1}$ and are therefore scaled down to a luminosity $200 \mathrm{pb}^{-1}$ in order to be directly comparable with results for the old very high- $p_{T}$ cuts. The relative efficiency of the two sets of cuts can be obtained by comparing the number of Drell-Yan events. As can be seen from table A.3, the new cuts greatly reduce the jet background above $M_{e e}=200 \mathrm{GeV} / c^{2}$ compared to the old very high- $p_{T}$ cuts, while still maintaining the selection efficiency of the old very high- $p_{T}$ cuts. 


\begin{tabular}{|c|c|c|}
\hline Variable & Standard Cuts & Old Very High- $p_{T}$ Cuts \\
\hline \hline Region & $=$ PEM & $=$ PEM \\
$E_{T}$ & $\geq 20 \mathrm{GeV}$ & $\geq 25 \mathrm{GeV}$ \\
PES $\eta$ & $1.2 \leq|\eta| \leq 2.0$ & $1.2 \leq|\eta| \leq 3.0$ \\
Nr Si Hits & $\geq 3$ & - \\
PHX $\left|z_{0}\right|$ & $\leq 60$ & - \\
\hline Had $/$ em & $\leq 0.05$ & $\leq 0.055+0.00045 \times E$ \\
Isolation $E_{T} / E_{T}$ & $\leq 0.1$ & $\leq 0.1$ \\
PEM $\chi_{3 \times 3}^{2}$ & $\leq 10$ & $\leq 10$ \\
PES $\Delta R$ & $\leq 3.0 \mathrm{~cm}$ & - \\
PES $5 \times 9 \mathrm{U}$ & $\geq 0.65$ & - \\
PES $5 \times 9 \mathrm{~V}$ & $\geq 0.65$ & - \\
\hline
\end{tabular}

Table A.2: The standard PEM high- $p_{T}$ cuts used by CDF in a kinematic region around the $Z^{0}$ pole and the old very high- $p_{T}$ PEM cuts used by the previous high mass dielectron analysis. The first section of the table list the kinematic and geometric cuts and the second section lists the identification cuts.

\begin{tabular}{|c|c|c|c|c|}
\hline \multirow{2}{*}{ Background Source } & \multicolumn{2}{|c|}{ Number of Events with $M_{e e} \geq 200 \mathrm{GeV} / c^{2}$} \\
\cline { 2 - 5 } & \multicolumn{2}{|c|}{ Central-Central } & \multicolumn{2}{c|}{ Central-Plug } \\
\cline { 2 - 5 } & New cuts & Old cuts & New Cuts & Old Cuts \\
\hline Drell-Yan & $16.3 \pm 1.3$ & 13.3 & $19.0 \pm 1.9$ & 19.1 \\
Jet & $0.05 \pm 0.05$ & 8.2 & $6.9 \pm 2.7$ & 29.4 \\
Di-Photon & $0.24 \pm 0.05$ & - & $0.9 \pm 0.1$ & - \\
EWK & $0.34 \pm 0.03$ & 0.2 & $1.1 \pm 0.2$ & 0.3 \\
\hline
\end{tabular}

Table A.3: Comparison of the new and the old very high- $p_{T}$ cuts. The number of events above $M_{e e}=200 \mathrm{GeV} / c^{2}$ passing the old cuts are taken from [10] and correspond to a luminosity of $200 \mathrm{pb}^{-1}$. The number of events passing the new cuts are therefore scaled to a luminosity of $200 \mathrm{pb}^{-1}$ for easier comparison. The di-photon background was not evaluated in [10]. 


\section{A.1 Central Electron Identification Cuts}

\section{CEM Isolation Cut}

The standard isolation cut is in terms of a ratio in which the isolation $E_{T}$ must be less than $10 \%$ of the electron $E_{T}$. This is fine at low energy $\left(E_{T} \sim 40 \mathrm{GeV}\right)$, however at high energy $\left(E_{T} \sim 100 \mathrm{GeV}\right)$, this allows the electron to have over $10 \mathrm{GeV}$ of isolation $E_{T}$. Typically the isolation $E_{T}$ for an electron at this energy will be much lower than this.

The isolation cut is about $98 \%$ efficient at low energies and the new cut should maintain this efficiency at high energies. Therefore, to determine what the value of the isolation $E_{T}$ cut will give a $98 \%$ efficiency at higher energies, electrons in Monte Carlo generated events are placed into bins of $E_{T}$. For each bin, the value of the isolation $E_{T}$ which is greater than the isolation $E_{T}$ of $98 \%$ of the electrons in that bin is obtained. These values of isolation $E_{T}$ are shown in figure A.1 and range from $\sim 4 \mathrm{GeV}$ at $E_{T}=50 \mathrm{GeV}$ to $\sim 10 \mathrm{GeV}$ at $E_{T}=450 \mathrm{GeV}$. The increase in the required value of isolation $E_{T}$ is approximately $1.6 \%$ of the electron $E_{T}$.

Therefore the cut is modified to have the form $E_{T}^{i s o l} \leq a+b \times E_{T} \mathrm{GeV}$, which is of a similar form as the isolation cut used in high- $p_{T}$ photon identification [45]. The parameter $b$ is chosen to be 0.02 . It is rounded up from 0.016 to 0.02 in order to be conservative and so ensure that the efficiency will not drop as the energy increases and also to be consistent with the choice made for the corresponding photon identification cut. The parameter $a$ is chosen to be $3 \mathrm{GeV}$ as then the cut is approximately equal to the ratio cut of 0.1 at around an $E_{T}$ of $40 \mathrm{GeV}$, a typical $E_{T}$ at the $Z^{0}$ pole. As can be seen from figure A.2, while the cut has a lower efficiency by about $2 \%$, the background rejection is about $50 \%$ while the previous cut's background rejection is very poor above an $E_{T}$ of $100 \mathrm{GeV}$.

\section{$E / p$ Cut}

As the resolution of the momentum measurement decreases with increasing energy for reasons stated in section 2.2 , the $E / p$ quantity becomes increasingly poorly measured as the electron energy increases. In the standard cuts, the $E / p$ cut is released if the track $p_{T}$ is greater than $50 \mathrm{GeV} / c$ to account for this. However this results in a slightly discontinuous efficiency vs $E_{T}$. This could sculpt the mass spectrum, as both signal and background events below $p_{T} \sim 50 \mathrm{GeV}$ are less likely to be selected than events above $p_{T} \sim 50 \mathrm{GeV}$, resulting in an artificial peak. Hence the cut is reformulated to account for the worsening $E / p$ resolution with a scaling with electron $E_{T}$ that is continuous.

The new cut is of the form $E / p \leq a+b \times E_{T}$ for $E_{T} \leq 100 \mathrm{GeV}$. For $E_{T}>100 \mathrm{GeV}$ the track $p_{T}$ is just required to be greater than $25 \mathrm{GeV} / c$. This value is chosen as the COT has sufficient resolution to measure any track with a $p_{T}$ up to $25 \mathrm{GeV} / c$ very well. The variable $b$ is chosen to be 0.015 as this gives a reasonable flat efficiency and background rejection across the $E_{T}$ range, as can be seen from figure A.3. In order for 


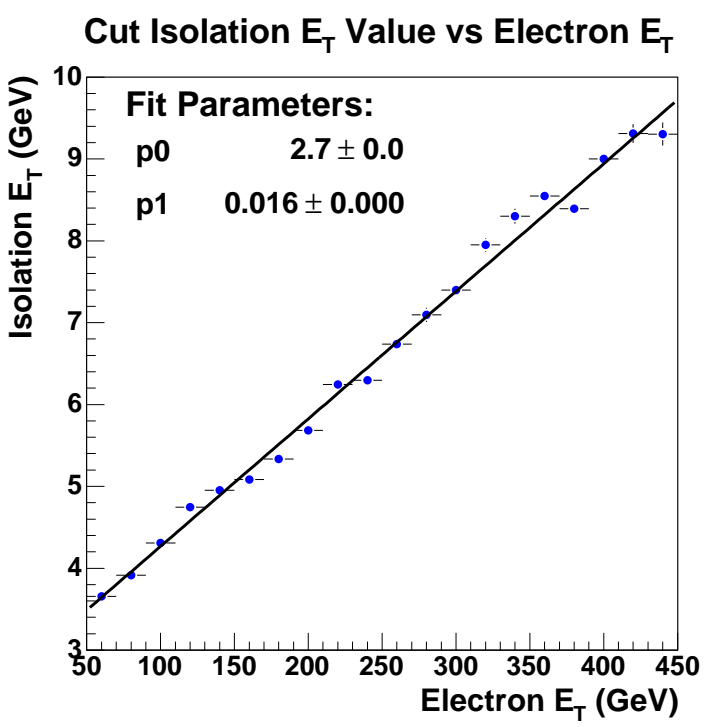

Figure A.1: The electron isolation $E_{T}$ which is greater than the isolation $E_{T}$ of $98 \%$ of the electrons in a given $E_{T}$ bin for Monte Carlo generated events. A straight line of the form $p_{0}+p_{1} \times x$ is fitted to the values and the fit parameters are displayed on the plot.
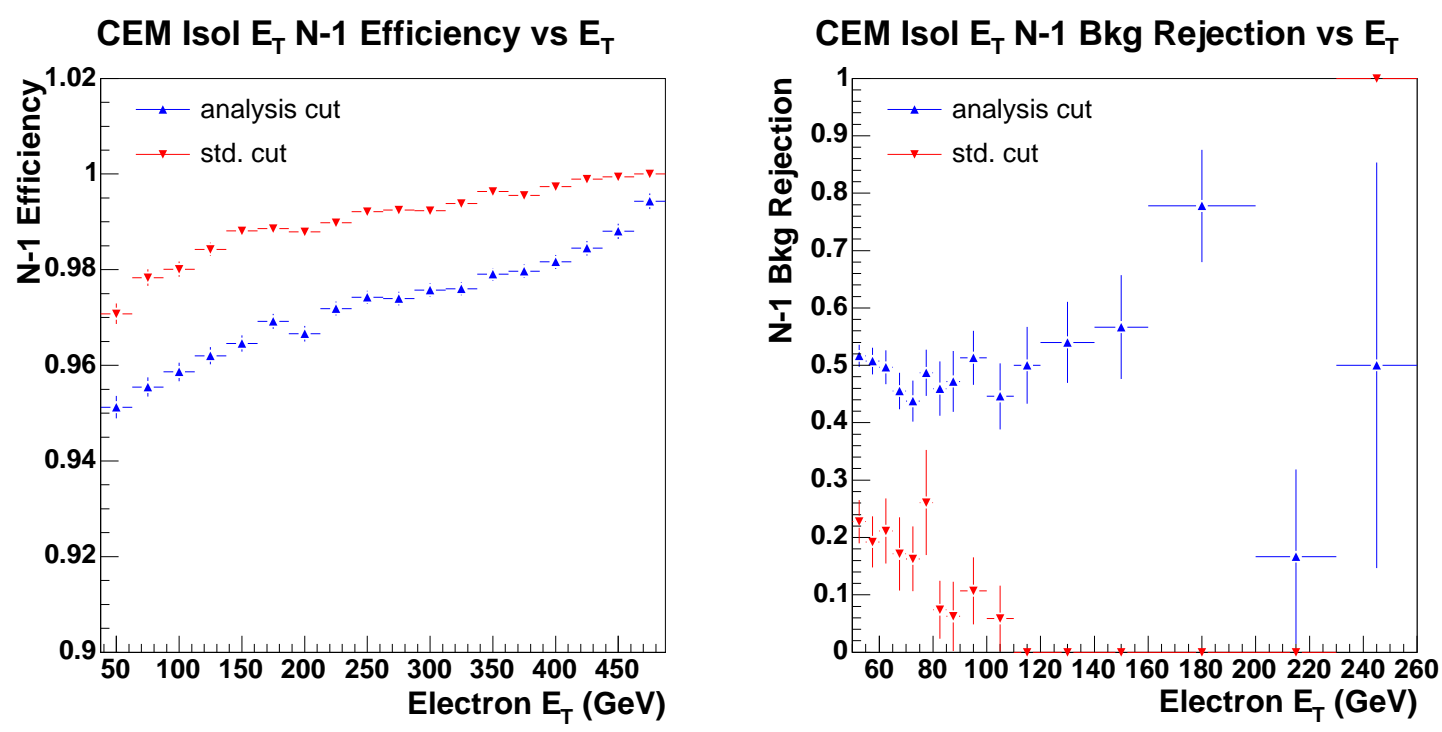

Figure A.2: The N-1 efficiency (left) and background rejection (right) of the isolation $E_{T}$ cut for central electrons compared to the standard cut. 
$E / p$ N-1 Efficiency vs $E_{T}$

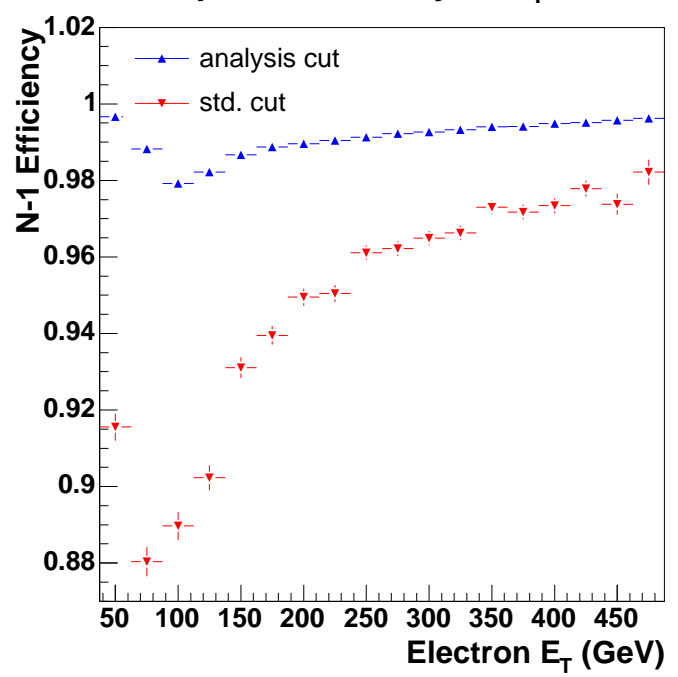

$\mathrm{E} / \mathrm{p} \mathrm{N}$-1 Bkg Rejection vs $\mathrm{E}_{\mathrm{T}}$

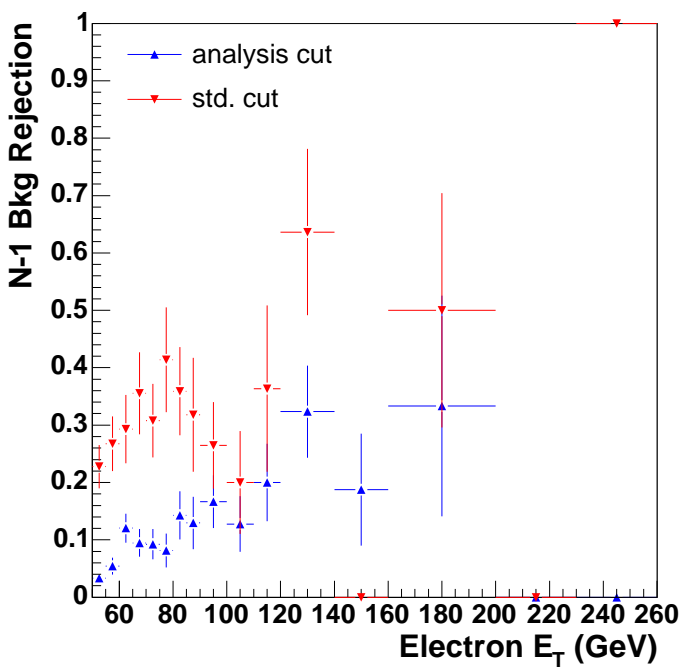

Figure A.3: The N-1 efficiency (left) and background rejection (right) of the $E / p$ cut for central electrons compared to the standard cut. The dips in the efficiency is an artifact of the track $p_{T}$ cut which is included as part of the acceptance and ensures that the $E / p$ is artificially very efficient at low mass as the track $p_{T}$ is already required to be above $15 \mathrm{GeV} / c$.

the cut to be continuous, $a+b \times E_{T}$ must equal 4 at $E_{T}=100 \mathrm{GeV}$ and therefore the parameter $a$ is taken to be 2.5 .

\section{Conversion Cut}

A conversion cut is added to the central leg of central-plug events. This is motivated by a desire to reduce the large di-photon background in the central-plug channel. In addition, a conversion cut will also reduce the jet background due to the high track multiplicity of jets. The conversion cut introduces an efficiency loss of about $4 \%$, however it reduces the non Drell-Yan background by about $50 \%$. As can be seen from the mass spectrum of anti-conversion sample in section 6.5. most electrons failing the conversion cut at high mass are either jet or di-photon background events. This cut is also well modeled in Monte Carlo, as can be seen from the good agreement between data and Monte Carlo efficiency estimates in section 5.1. Figure A.4 shows that its efficiency and background rejection is appproximately flat as a function of $E_{T}$. The cut is not applied to electrons of central-central events as the di-photon background and jet backgrounds in this channel are already sufficiently small and so applying the cut would result in a needless efficiency loss. 

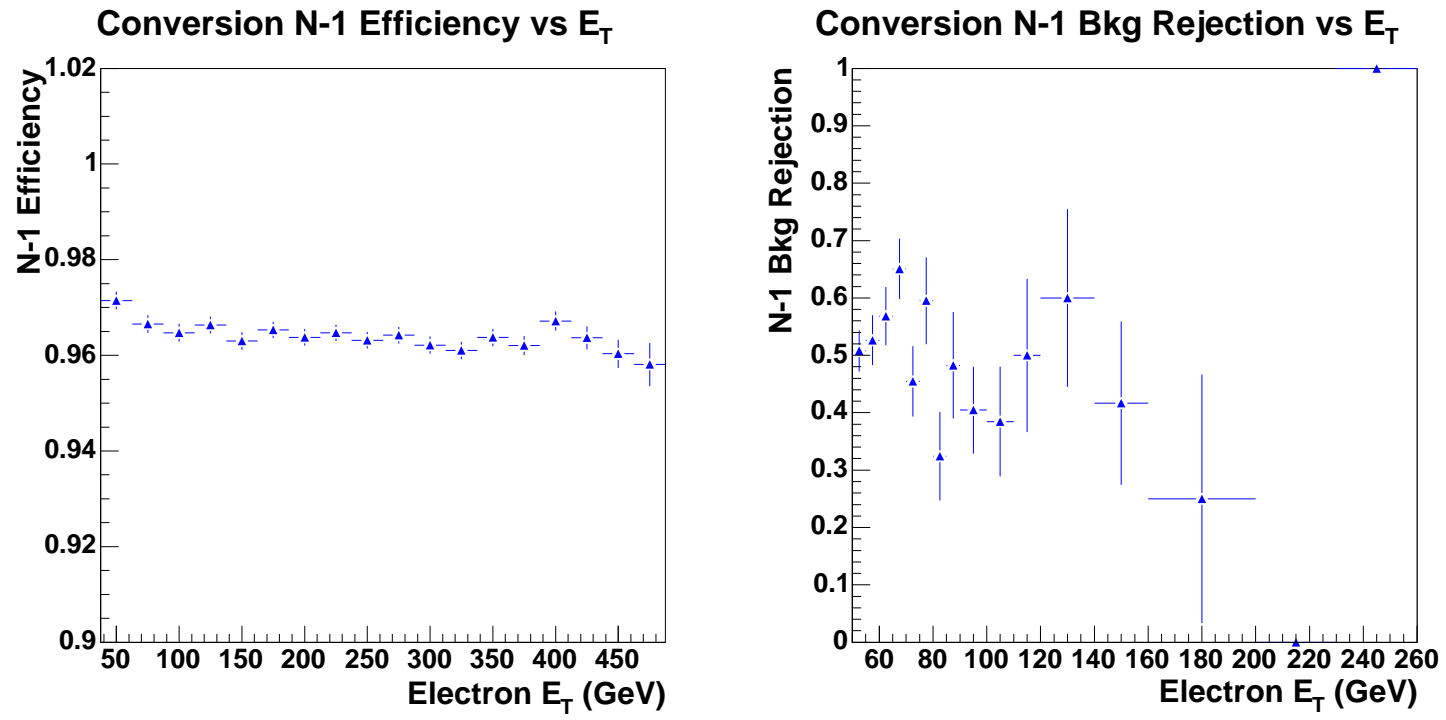

Figure A.4: The N-1 efficiency (left) and background rejection (right) of the conversion cut for central electrons.

\section{$\chi_{\text {strip }}^{2}$ Cut}

As can be seen from figure A.5 the $\chi_{\text {strip }}^{2}$ cut loses efficiency in the interesting high $E_{T}$ region where the backgrounds are the lowest. Therefore the cut is not used in this analysis.

\section{$L_{S h r}$ Cut}

As can be seen from figure A.6, the $L_{S h r}$ cut has a high efficiency and good background rejection at high mass. Therefore it is retained unmodified for new very high- $p_{T}$ cuts.

\section{CES $\Delta X$ and $\Delta Z$ Cuts}

The CES $\Delta X$ and $\Delta Z$ cuts have an efficiency of $\sim 100 \%$ in the high $E_{T}$ region. They are retained to ensure that the track is well matched to the CES cluster. As the charge of very high- $p_{T}$ electrons is poorly measured due to straightness of the track, the $\Delta X$ cut is made symmetric with an absolute maxium of $3 \mathrm{~cm}$ permitted regardless of the track charge. The $\Delta Z$ cut is relaxed to $5 \mathrm{~cm}$ following the cuts of the previous high mass analysis. Figures A.7 and A.8 show the N-1 efficencies and background rejections for the CES $\Delta X$ and $\Delta Z$ cuts respectively. 

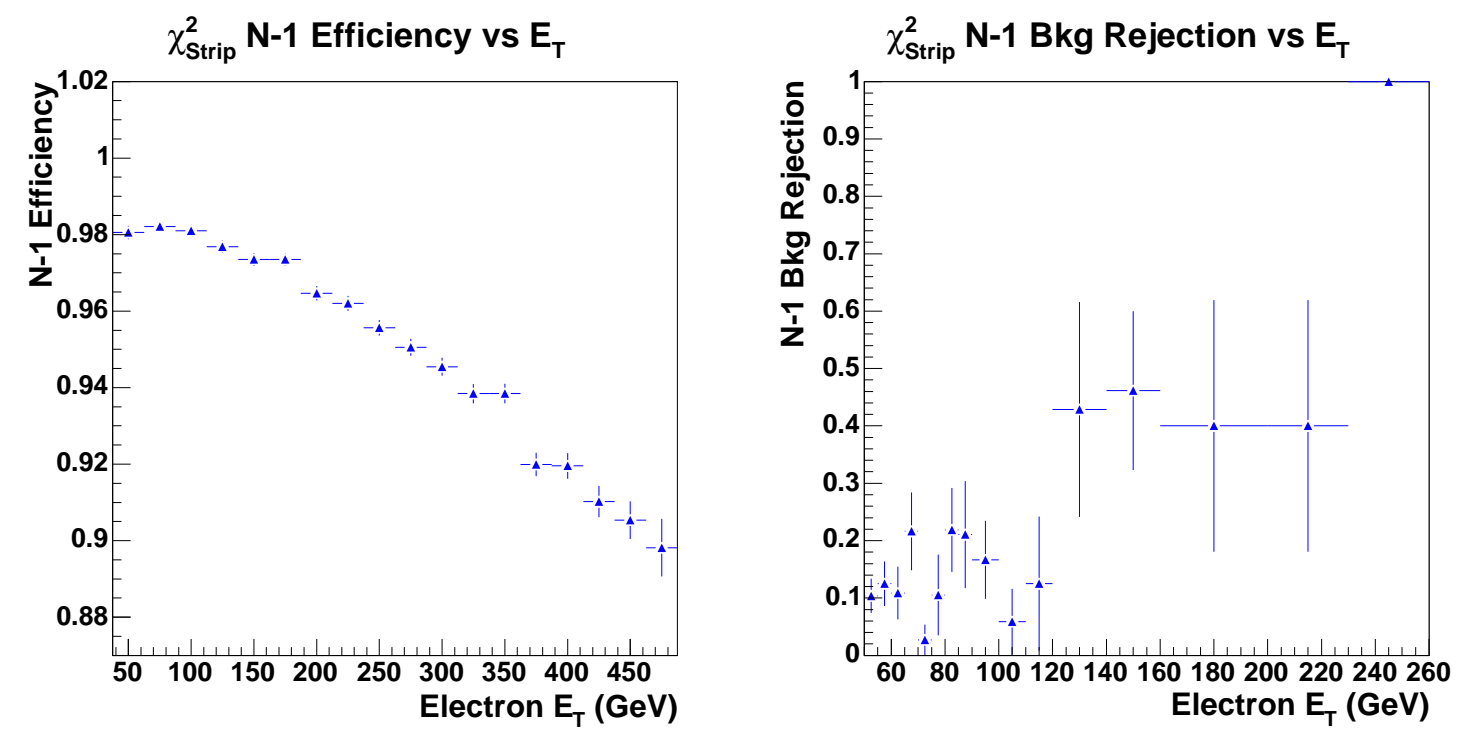

Figure A.5: The N-1 efficiency (left) and background rejection (right) of the $\chi_{\text {Strip }}^{2}$ cut for central electrons.
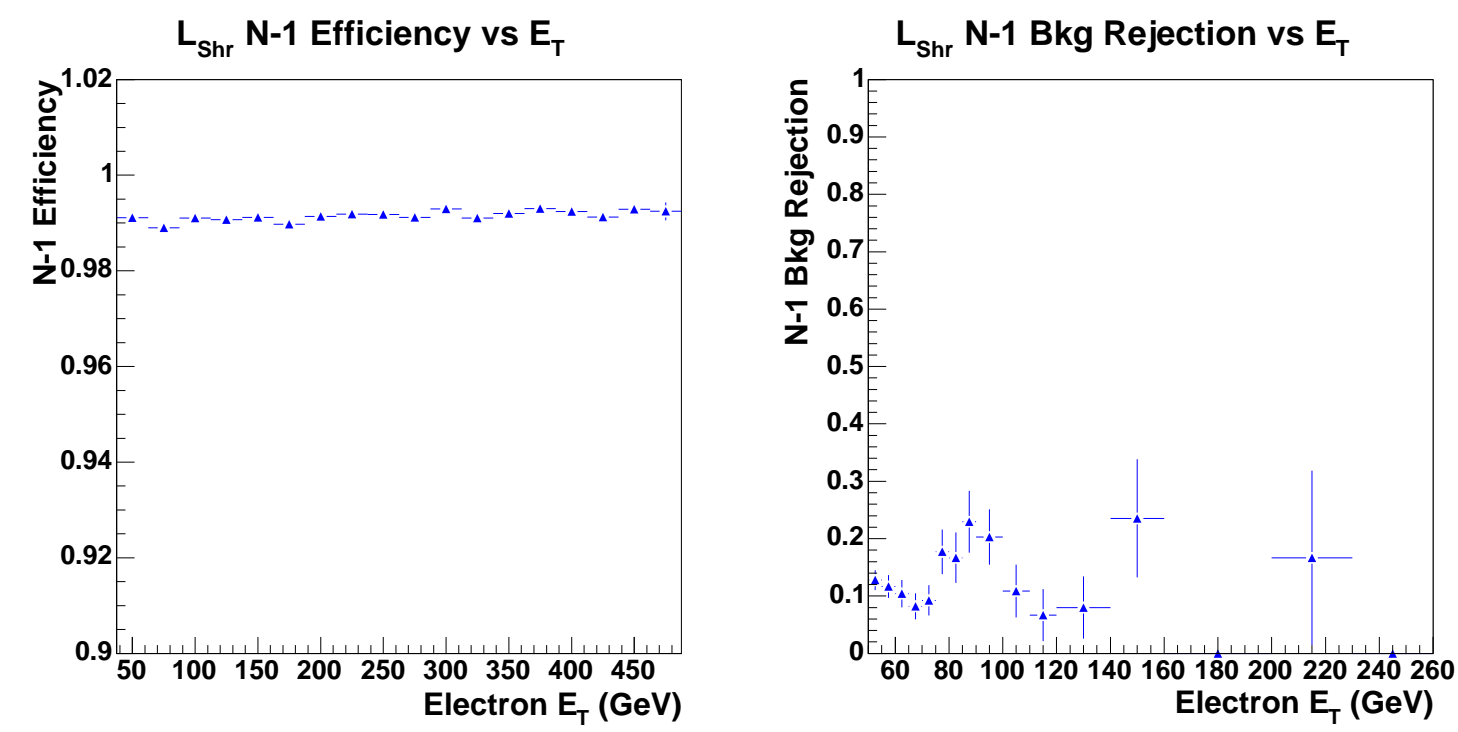

Figure A.6: The N-1 efficiency (left) and background rejection (right) of the $L_{S h r}$ cut for central electrons. 

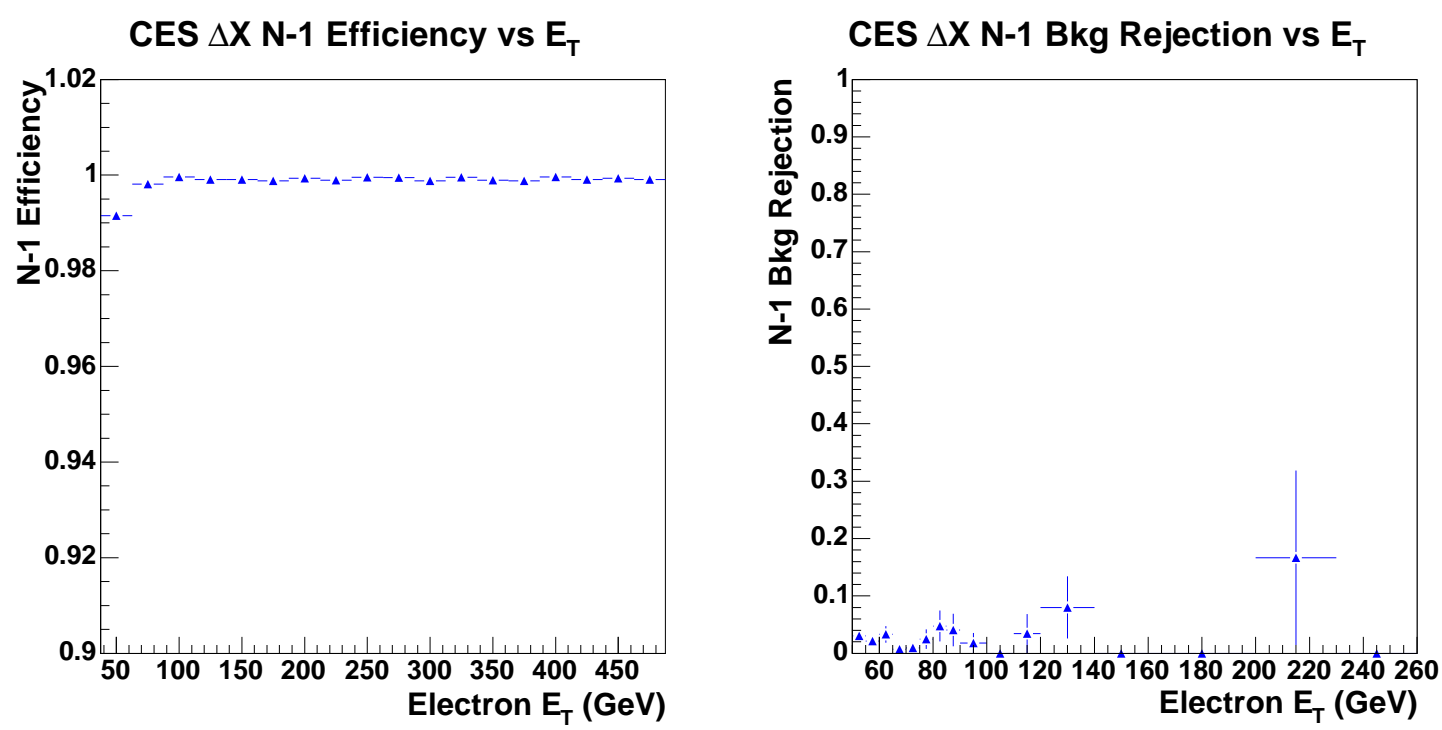

Figure A.7: The N-1 efficiency (left) and background rejection (right) of the CES $\Delta X$ cut for central electrons.
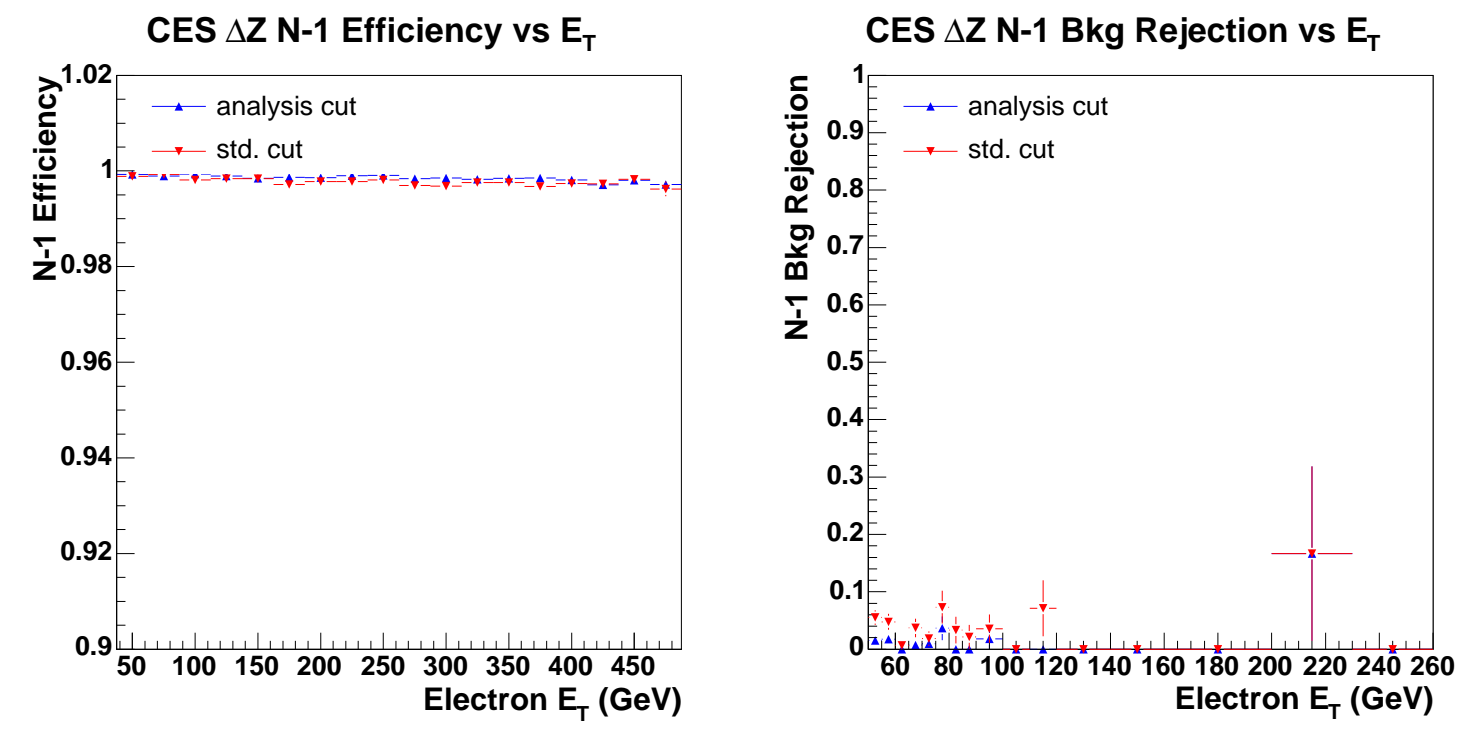

Figure A.8: The N-1 efficiency (left) and background rejection (right) of the CES $\Delta Z$ cut for central electrons compared to the standard cut. 


\section{COT Axial and Stereo Segments Cuts}

These cuts both have an efficiency of $\sim 100 \%$ and a background rejection of $\sim 0 \%$. As they have neglible effect on both signal and background events and any cross-check they could provide will be covered by the $E / p$, track $p_{T}$, CES $\Delta X$ and $\Delta Z$ cuts, these cuts were rejected for use in this analysis.

\section{CEM Had/Em Cut}

Substantial work had already gone into defining the had/em cut in previous CDF studies which involved the use of more detailed detector simulation than is normally used. Therefore it is kept unchanged for this analysis.

\section{A.2 Plug Electron Identification Cuts}

\section{PEM Isolation Cut}

The standard PEM isolation cut, like the standard CEM cut discussed in section A.1 is a ratio cut where the isolation $E_{T}$ must be less than $10 \%$ of the electron $E_{T}$. Again for reasons discused in A.1, the cut is changed to the form $E_{T}^{i s o l} \leq a+b \times E_{T} \mathrm{GeV}$, with $b$ chosen to be 0.02 to be consistent with the photon identification cuts. Due to the nature of the plug calorimeter, plug electrons will typically have a lower isolation $E_{T}$ than central electrons and so the value of $a$ is chosen to be $1.6 \mathrm{GeV}$. The precise value of $1.6 \mathrm{GeV}$ is chosen so that the cut exactly matches the corresponding photon identification cut. Figure A.9 shows the N-1 efficiency and background rejection of this cut and the standard cut. While the efficiency is reduced by about $2 \%$ compared to the standard cut, the background rejection is around $40 \%$ to $60 \%$, while the standard cut has a background rejection of $<10 \%$.

\section{PEM $\chi_{3 \times 3}^{2}$ Cut}

The standard PEM $\chi_{3 \times 3}^{2}$ cut is $\chi_{3 \times 3}^{2} \leq 10$. However, as can be seen from figure A.10, there is a sharp $2 \%$ jump in efficiency as the energy increases. The $\chi_{3 \times 3}^{2}$ variable is not well modeled in Monte Carlo, due in part to its sensitivity to minor variations in the energy calibrations 1 . It is not known whether this large increase in efficiency is real or not. To reduce the problem, the $\chi_{3 \times 3}^{2}$ cut is loosened to $\leq 25$ to make the cut already $\sim 98 \%$ efficient at the $Z^{0}$ pole. From figure A.10, the N-1 efficiency is approximately flat as function of $E_{T}$. However the background rejection is generally lower than the previous cut, but at $E_{T}$ greater than $100 \mathrm{GeV}$ the background rejection estimates are consistent with each other, within the large errors of the measurement.

\footnotetext{
${ }^{1}$ It has been found that applying different versions of the calibrations can change some electrons PEM $\chi_{3 \times 3}^{2}$ values from $<10$ to $>20$.
} 

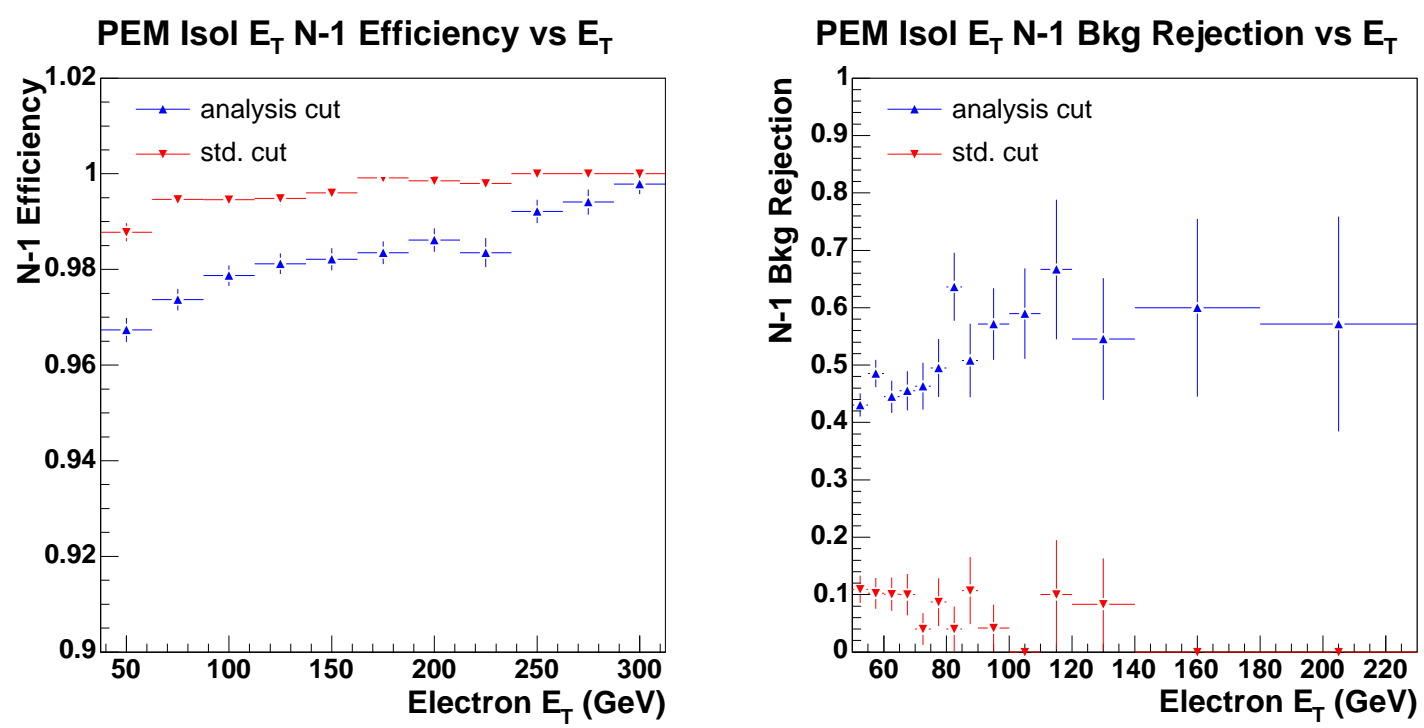

Figure A.9: The N-1 efficiency (left) and background rejection (right) of the isolation $E_{T}$ cut for plug electrons compared to the standard cut.
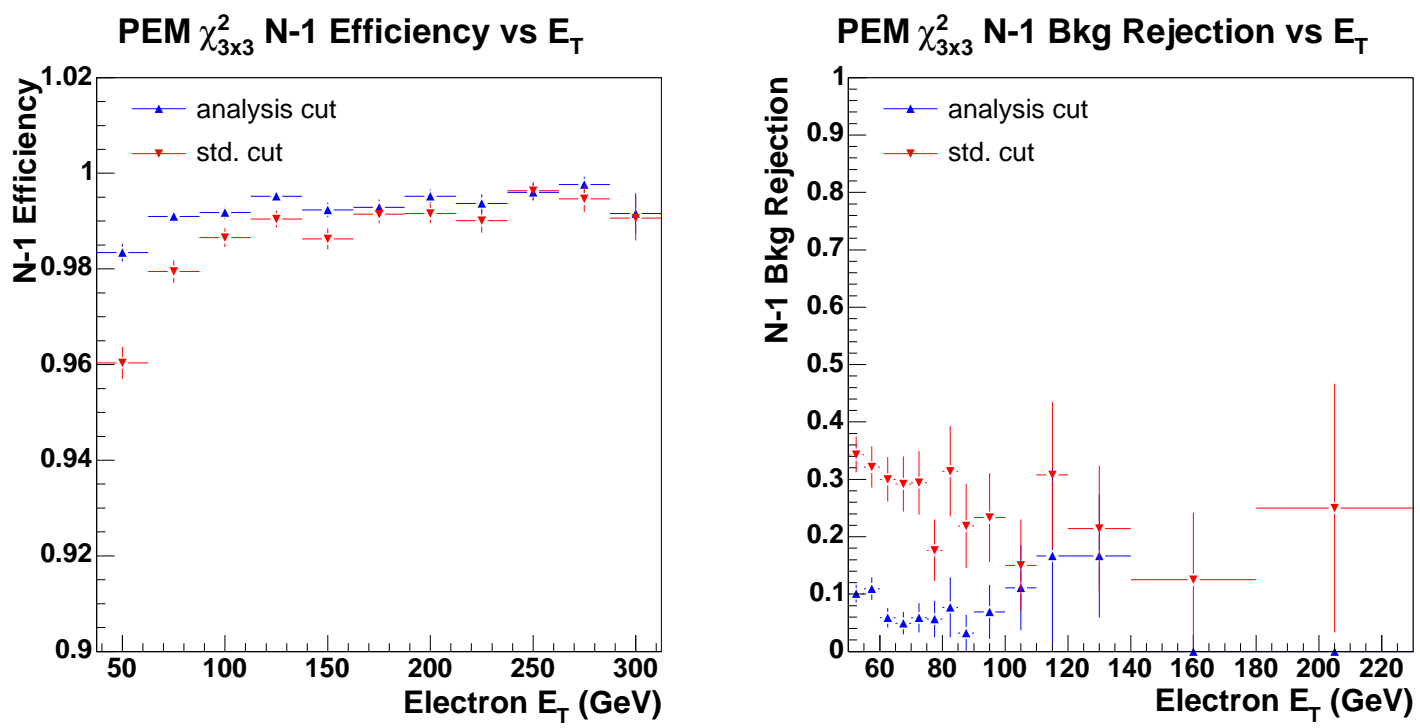

Figure A.10: The N-1 efficiency (left) and background rejection (right) of the PEM $\chi_{3 \times 3}^{2}$ cut for plug electrons compared to the standard cut. 

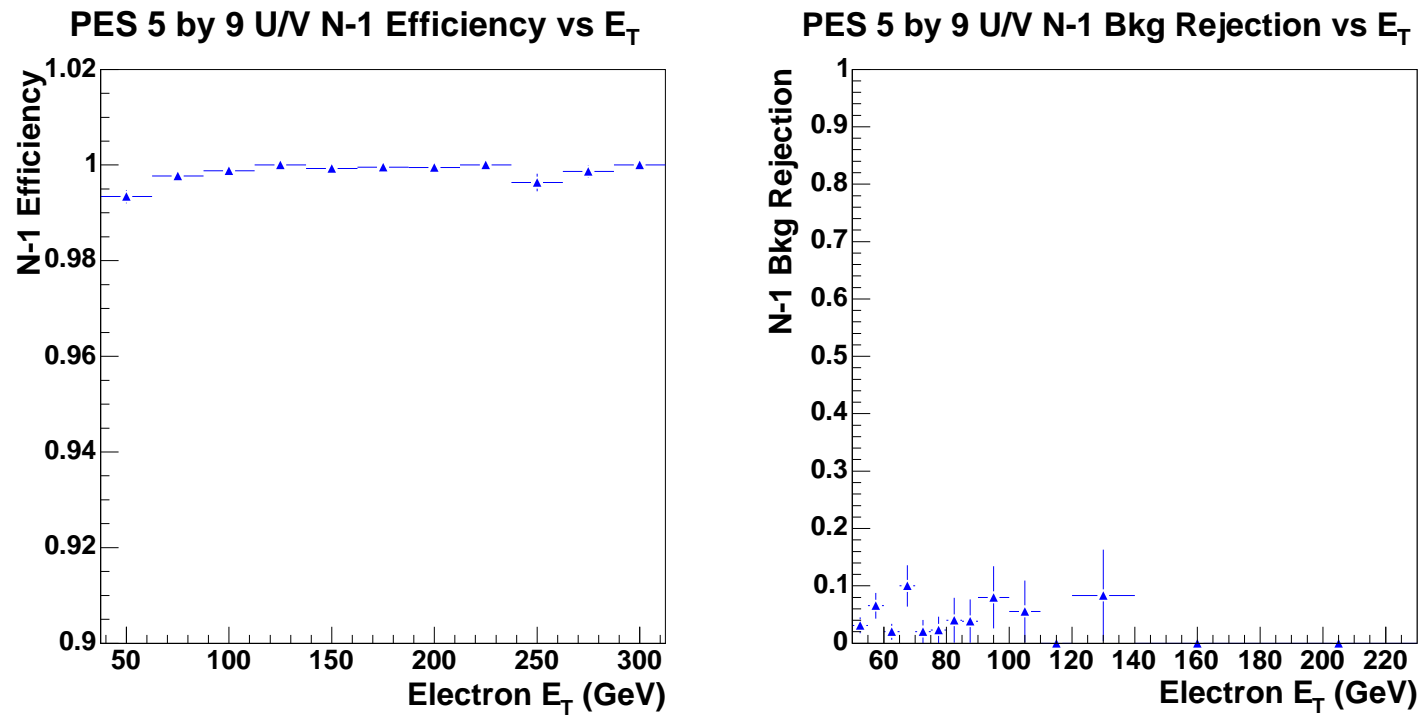

Figure A.11: The N-1 efficiency (left) and background rejection (right) of the PES $5 \times 9$ $\mathrm{U} / \mathrm{V}$ cut for plug electrons.

\section{PES $5 \times 9 \mathrm{U} / \mathrm{V}$ Cuts}

The PES $5 \times 9 \mathrm{U} / \mathrm{V}$ cuts are cuts on the electron shower profile in the PES scintillator strips. Although they are two separate cuts, they are counted as a single cut for the $\mathrm{N}-1$ efficiencies and background rejections due to their similarity. As can be seen in figure A.11, these cuts do very little to the background and so they are not used in this analysis despite their high efficiency.

\section{PEM Had/Em Cut}

Like the CEM had/em cut, substantial work had already be done in previous CDF studies to produce an optimised plug had/em cut and due to the difficult of repeating this work, the cut was left unchanged for this analysis except for the addition of a logarithmic energy dependence for energies above than $100 \mathrm{GeV}$. This logarithmic dependence had been recommended by a previous CDF study on the PEM Had/Em cut.

\section{PES $\Delta R$ Cut}

The $\Delta R$ cut is a separation cut on the shower maximum position as measured by the PES and the shower position as measured by the PEM 3x3 fit. As figure A.12 shows, the cut rejects very little background for its efficiency cost and so is not used in this analysis. 

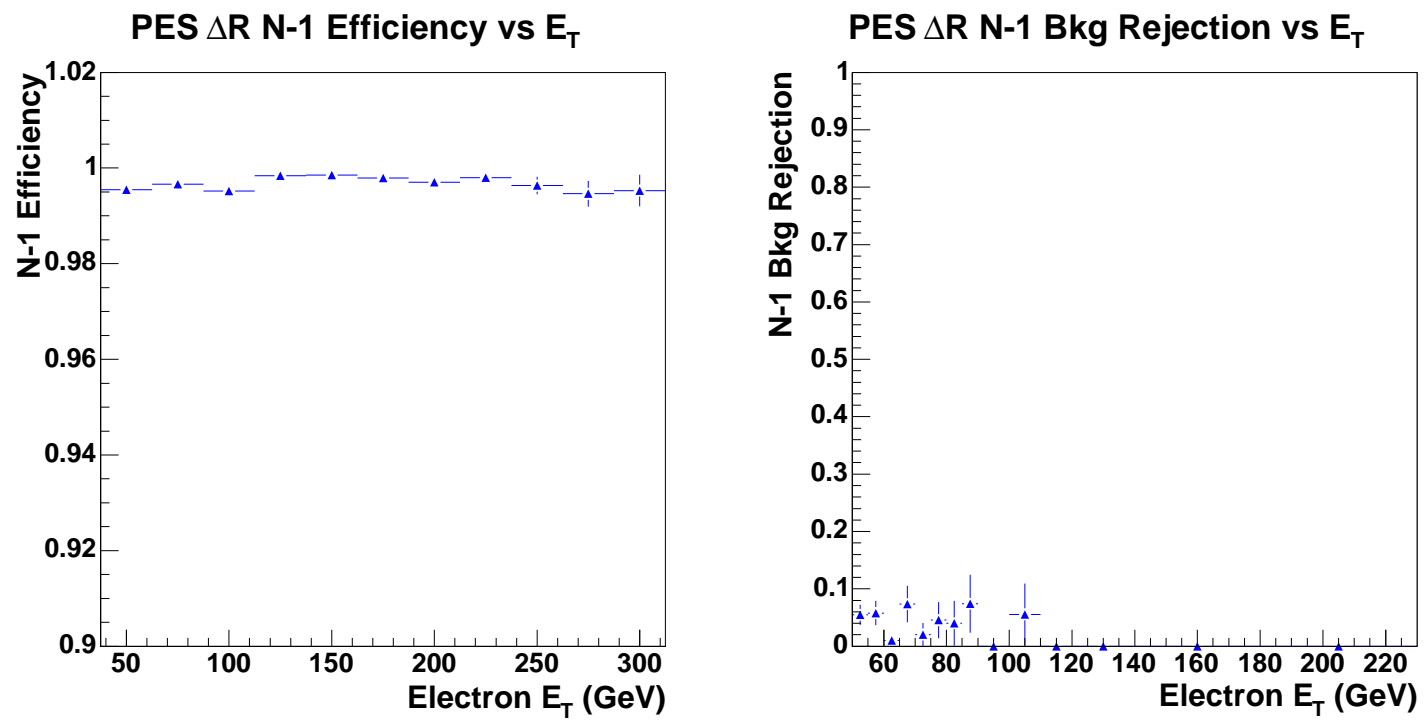

Figure A.12: The N-1 efficiency (left) and background rejection (right) of the PEM $\Delta R$ cut for plug electrons.

\section{Phoenix Tracking Requirements}

A major consideration in setting the selection requirements is whether to require to that plug electrons have an associated Phoenix track or not. The Phoenix tracking algorithm is discussed in more detail in section 4.2.3 and makes use of the silicon tracking system. Requiring a Phoenix track greatly reduces the non Drell-Yan background in centralplug events to levels comparable to that of central-central events. However it also greatly reduces the acceptance and event selection efficiency, not least due to requiring events to have $\eta$ approximately less than 2. Phoenix tracking also requires the run to be marked good for silicon which reduces the available luminosity by $9 \%$.

The fraction of the total background which is non Drell-Yan falls rapidly until it is almost negligible at $M_{e e} \sim 500 \mathrm{GeV} / c^{2}$. However di-electron events become increasingly central as $M_{e e}$ increases so the fraction of genuine di-electron events outside the acceptance of the Phoenix tracking algorithm also falls with increasing invariant mass. However it is expected that at lower invariant masses requiring a Phoenix track will increase the sensitivity of the analysis whereas at higher invariant masses requiring a Phoenix track will reduce the sensitivity. In order to evaluate whether it is better to require a Phoenix track or not, the quantities $S / \sqrt{B}$ and $S / \sqrt{S+B}$ are used as guides. Maximising $S / \sqrt{B}$ will allow a better limit to be extracted, while maximising $S / \sqrt{S+B}$ will increase the analysis sensitivity to discovering new physics. In order to evaluate the relative effect on the two quantities, it is assumed that requiring a Phoenix track eliminates $100 \%$ of the di-photon and jet backgrounds and that the electroweak background has a neglible effect, assumptions which are valid to a good approximation. It is also assumed that the new physics signal events have the same 
geometric and kinematic distribution as the Drell-Yan events and therefore the fraction of signal events lost by requiring a Phoenix track is equal to the fraction of the DrellYan events lost.

Requiring a Phoenix track has the same effect as not requiring a Phoenix track when

$$
S / \sqrt{B+Z}=x S / \sqrt{y B+x Z}
$$

where $x$ and $y$ are respectively the fraction of signal events and the fraction of jet and di-photon background events remaining after requiring a Phoenix track while $\mathrm{Z}$ and B are respectively the number of Drell-Yan and jet+di-photon background events. Taking $y$ to be 0 , requiring a Phoenix track gives a better limit when

$$
\begin{array}{r}
x>Z /(B+Z) \\
1-x<B /(B+Z) .
\end{array}
$$

Therefore, when the fraction of signal lost by requiring a Phoenix track $(1-x)$ is less than the fraction of jet and di-photon backgrounds to the total background, requiring a Phoenix track maximises $S / \sqrt{B}$. For the case of maximising $S / \sqrt{S+B}$, the relationship becomes

$$
\begin{array}{r}
x>(S+Z) /(S+B+Z) \\
1-x<B /(S+B+Z)
\end{array}
$$

and so requiring a Phoenix track maximises the potential of discovery when the fraction of signal lost is less than the fraction of the jet and di-photon backgrounds to the total background and signal. Figure A.13 shows the fraction of the jet and di-photon backgrounds to the total background in a mass window equal to the width of a narrow resonance in the CDF detector around a given mass point. Also shown in A.13 is the fraction of the jet and di-photon backgrounds to the total background and signal in a mass window equal to the width of a narrow resonance with the signal normalised so that there is a total of 4 signal events in the mass window, which roughly corresponds to a $\sigma$.Br of about $0.02 \mathrm{pb}$. A larger number of signal events will lower the point where it becomes better not to use Phoenix tracking and a smaller number of signal events is beyond the sensitivity of this analysis in the regions where the jet and di-photon backgrounds are significant. Overlaid on both figures is the fraction of events lost by requiring a Phoenix track. This is estimated from a $Z^{\prime}$ Monte Carlo simulation taking into account that Phoenix tracking requires the silicon detector to be operational at the time of data taking and this will reduce the available luminosity. From figure A.13 it can be seen that, in order to set the best limit, it is better to require a Phoenix track below invariant masses of $230 \mathrm{GeV} / c^{2}$ while it is better not to require one above $230 \mathrm{GeV} / c^{2}$. To maximise the potential for discovery it is better to not to require a Phoenix track at all invariant masses. At almost all points in the limit setting range of $200-950 \mathrm{GeV} / c^{2}$, requiring that a plug electron has an associated Phoenix track reduces the sensitivity of the analysis. Therefore plug electrons are not required to have an associated Phoenix track in this analysis. 
Fraction of Jet and $\gamma \gamma$ Backgrounds of the Total Bkg.

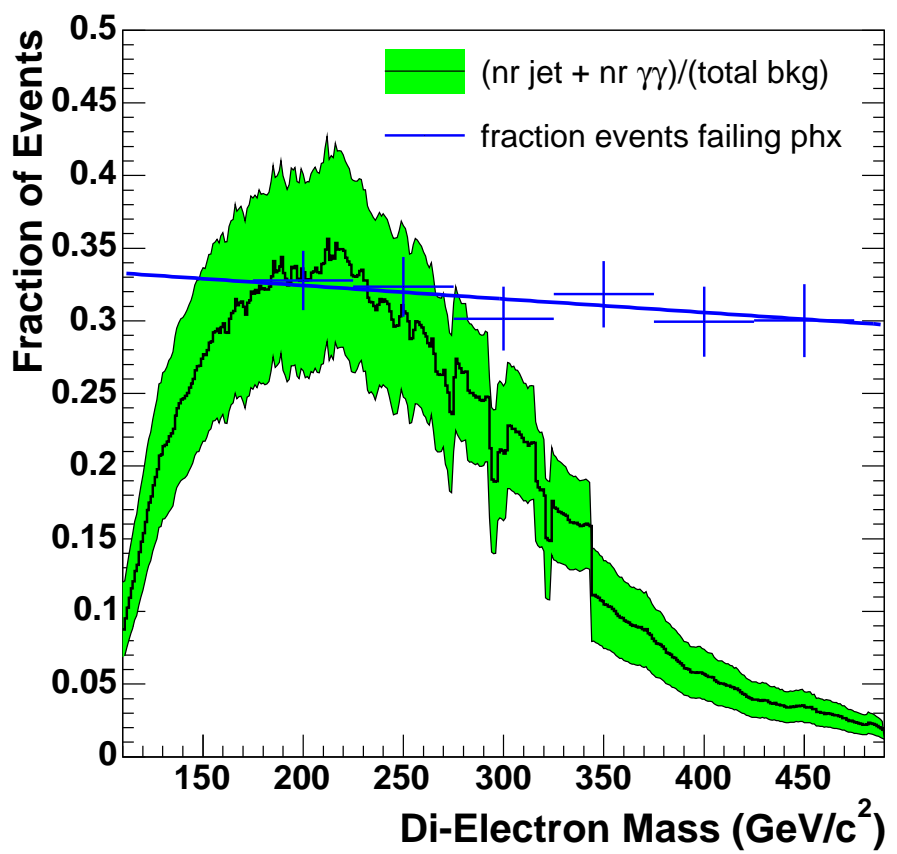

Fraction of Jet and $\gamma \gamma$ Backgrounds of the Total Bkg. + Sig.

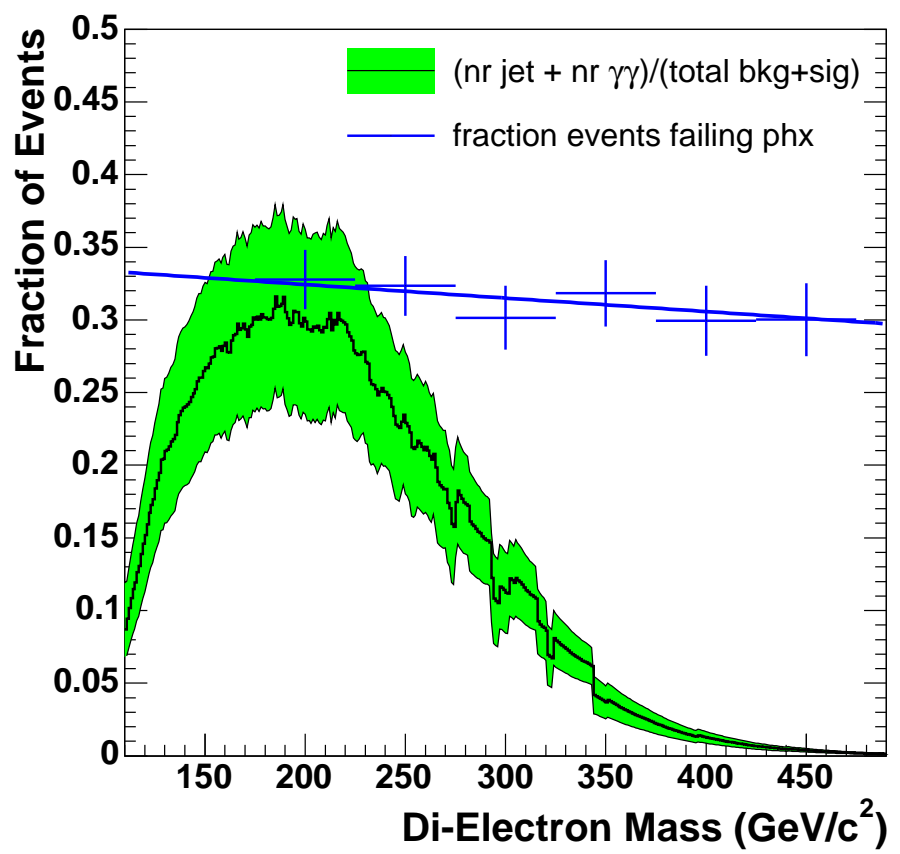

Figure A.13: The upper (lower) plot shows the fraction of jet and di-photon events to the total background (total background and signal) in a window corresponding to a narrow resonance centred on the mass point. The signal is normalised so that there is a total of 4 events in the mass window. Also overlaid on both plots is the fraction of signal lost when requiring a Phoenix track, together with a linear fit. 


\section{Bibliography}

[1] The SNO Collaboration, "Direct Evidence for Neutrino Flavor Transformation from Neutral-Current Interactions in the Sudbury Neutrino Observatory", Phys. Rev. Lett. 89, 011301 (2002).

[2] F. Halzen and A. D. Martin, "Quarks and Leptons", John Wiley and Sons, NY (1984).

[3] S. L. Glashow, "Partial-symmetries of weak interactions", Nucl. Phys. 22, 579 (1961);

S. Weinberg, “A Model of Leptons", Phys. Rev. Lett. 19, 1264 (1967);

A. Salam, in Proceedings of the 8th Nobel Symposium on Elementary Particle Theory, Relativistic Groups and Analyticity, edited by N. Svartholm, p. 367 (1968).

[4] W.-M. Yao et al., "Review of Particle Physics", J. Phys. G: Nucl. Part. Phys. 33, $1(2006)$.

[5] T. Sjöstrand et al., "High-Energy-Physics Event Generation with Pythia 6.1", Computer Phys. Commun. 135, 238 (2001).

[6] G. Corcella et al., "HERWIG 6.5", J. High Energy Phys. 0101, 010, (2001).

[7] G. Altarelli, "Partons in quantum chromodynamics", Phys. Rep. 81, 1, 1982.

[8] S. D. Drell and T.-M. Yan, "Massive Lepton-Pair Production in Hadron-Hadron Collisions at High Energies", Phys. Rev. Lett. 25, 316 (1970).

[9] D. Acosta et al. (The CDF Collaboration), "First Measurements of Inclusive $W$ and $Z$ Cross Sections from Run II of the Tevatron Collider", Phys. Rev. Lett. 94, 091803 (2005).

[10] A. Abulencia et al. (The CDF Collaboration), "Search for New High Mass Particles Decaying to Lepton Pairs in p anti-p Collisions at $\sqrt{s}=1.96 \mathrm{TeV}$ ", Phys. Rev. Lett. 95, 252001 (2005).

[11] G. Arnison et al. (The UA1 Collaboration), "Experimental Observation of Lepton Pairs of Invariant Mass Around 95 ,GeV/c 2 at the CERN SPS Collider", Phys. Lett. B 126, 398, (1983). 
G. Banner et al. (The UA2 Collaboration), "Evidence for $Z^{0} \rightarrow e^{+} e^{-}$at the CERN pp Collider", Phys. Lett. B 129, 130, (1983).

[12] G. 't Hooft, "Introduction to String Theory", Lectures given at Utrecht University in 2004, http://www.phys.uu.nl/ thooft/lectures/string.html.

[13] S.P. Martin, “A Supersymmetry Primer”, hep-ph/970956, (1997).

[14] P. Renton, "Electroweak Interactions", CUP (1990).

[15] S. Weinberg, "Implications of dynamical symmetry breaking: An addendum", Phys. Rev. D 19, 1277, (1979);

L. Susskind, "Dynamics of spontaneous symmetry breaking in the Weinberg-Salam theory", Phys. Rev. D 20, 2619, (1979);

E. Eichten and K. Lane, "Dynamical breaking of weak interaction symmetries", Phys. Lett. B 90, 125, (1980).

[16] G. Bertone, D. Hooper, J. Silk, "Particle Dark Matter: Evidence, Candidates and Constraints", Phys. Rept. 405, 279, (2005).

[17] P. J. E. Peebles, B. Ratra, "The Cosmological Constant and Dark Energy", Rev. Mod. Phys. 75, 559, (2003).

[18] M. Cvetic and P. Langacker, "New gauge bosons from string models", Mod. Phys. Lett. A 111247 (1996).

[19] D. London and J. L. Rosner, "Extra Gauge Bosons in E(6)", Phys. Rev. D 34, 1530, (1986).

[20] C. Ciobanu et. al., " $Z$ ' Generation with PYTHIA", CDF Public Note 7755, (2005).

[21] K. Lane, "Technicolor 2000", hep-ph/0007304, (2000).

[22] K. Lane and S. Mrenna, "The Collider Phenomenology of Technihadrons in the Technicolor Straw Man Model”, Phys. Rev. D 67, 115011, (2003).

[23] L. Randall and R. Sundrum, "A Large Mass Hierarchy from a Small Extra Dimension", Phys. Rev. Lett. 83, 3370 (1999).

[24] N. Arkani-Hamed, S. Dimopoulos, G. Dvali, "The hierarchy problem and new dimensions at a millimeter", Phys. Lett. B 429, 263, (1998).

[25] H. Davoudiasl, J.L. Hewett, T.G. Rizzo, "Phenomenology of the Randall-Sundrum Gauge Hierarchy Model", Phys. Rev. Lett. 84, 2080 (2000).

[26] B. C. Allanach et al., "Exploring Small Extra Dimensions at the Large Hadron Collider", J. High Energy Phys. 0212, 039, (2002). 
[27] A. Abulencia et al. (The CDF Collaboration), "Search for $Z^{\prime} \rightarrow$ e+ e- Using Dielectron Mass and Angular Distribution", Phys. Rev. Lett. 96, 211801 (2006).

[28] R. Brun and F. Rademakers, "ROOT - An Object Oriented Data Analysis Framework", http://root.cern.ch/.

[29] D. Acosta et al. (The CDF Collaboration), "Measurement of the J/psi meson and b-hadron production cross sections in $\mathrm{p} \bar{p}$ collisions at $\sqrt{s}=1960 \mathrm{GeV}$ ", Phys. Rev. D 71, 032001, (2005);

R. Blair et al. (The CDF Collaboration), "The CDFII Detector Technical Design Report", FERMILAB-PUB-96-390-E (1996).

[30] J. Marriner, "Stochastic Cooling Overview", Nucl. Inst. Meth. A 532, 11, (2004).

[31] I. Meshkov and A. Sidorin, "Electron Cooling", Nucl. Inst. Meth. A 532, 119, (2004).

[32] A. Sill et al. (The CDF Collaboration), "CDF Run II silicon tracking projects", Nucl. Inst. Meth. A 447, 1, (2000).

[33] COT Aging Committee (CDF), http://fcdfwww.fnal.gov/internal/upgrades/cot/

[34] L. Balka et. al., "The CDF Central Electromagnetic Calorimeter", Nucl. Inst. Meth. A 267, 272, (1988).

[35] M. Albrow et. al., "The CDF plug upgrade electromagnetic calorimeter: test beam results", Nucl. Inst. Meth. A 480, 524, (2002).

[36] D. Acosta et al. (The CDF Collaboration), "The CDF Cherenkov Luminosity Monitor", Nucl. Inst. Meth. A 461, 540, (2001).

[37] S. Klimenko, J. Konigsberg, and T.M. Liss, "Averaging of the inelastic cross sections measured by the CDF and the E811 experiments", FERMILAB-FN-0741 (2003).

[38] The CDF Collaboration, "The CDF Run II Software" http://cdfcodebrowser.fnal.gov/CdfCode/

[39] C. Ciobanu, J. Goldstein, S. Harper, T. Junk, J. Lee, G. De Lentdecker, K. McFarland G. Veramendi, "High Pt Dielectron Mass and Angular Distribution for $Z$ ' searches", CDF Internal Note 7584, (2005).

[40] The Data Quality Monitoring Group's page for Good Run List v10 http://www-cdf.fnal.gov/internal/dqm/goodrun/v10/goodv10.html.

[41] GEANT 3, "Detector Description and Simulation Tool", CERN Program Library Long Writeup W5013 (1993). 
[42] H. L. Lai et al., "Global QCD Analysis of Parton Structure of the Nucleon: CTEQ5 Parton Distributions", Eur. Phys. J C 12, 375, (2000).

[43] M. L. Mangano, M. Moretti, F. Piccinini, R. Pittau and A. Polosa, "ALPGEN, a generator for hard multiparton processes in hadronic collisions", J. High Energy Phys. 0307, 001, (2003).

[44] U. Baur and E. L. Berger, "Probing the WW $\gamma$ vertex at the Fermilab Tevatron Collider", Phys. Rev. D 41, 1476, (1990).

[45] T. Berry, R. Culbertson and S-M Wynne, "Search for a High-Mass Diphoton State and Limits on Randall-Sundrum Gravitons at CDF", CDF Public Note 8423, (2006).

T. Berry, R. Culbertson and S-M Wynne, "Search for Randall Sundrum Gravitons in $1155 \mathbf{~ p b}^{-\mathbf{1}}$ Run II High Mass Diphoton Data", CDF Internal Note 8359, (2006).

[46] Ben Brau, Antonio Boveia and David Stuart, "Search for Massive Resonances Decaying to $Z^{0} Z^{0}$ in the Final State eee", CDF Public Note 8419, (2006).

[47] A. Wyatt, B. Heinemann, "Correction for Leakage Energy in the Central and Plug Calorimeters in Run II", CDF Internal Note 6167, (2002).

[48] L. Nodulman, "Curvature Corrections for 5.3.1", CDF Internal Note 6971, (2004).

[49] J. Goldstein, S. Harper, B. Heinemann, G. Manca and P. Renton, "Reconstructing the Plug Electron Energy in 5.3.3", CDF Internal Note 7687, (2005).

[50] K. Yasuoka, S.Mikamo, T.Kamon, A.Yamashita, and the CDF NW WEDGE GROUP, "Response Maps of the CDF Central Electromagnetic Calorimeter with Electrons", Nucl. Inst. Meth. A 267, 315, (1988).

[51] B. Heinemann, University of Liverpool, private conversation.

[52] R.G. Wagner, "Understanding and Using Lshr", CDF Internal Note 6249, (2003).

[53] W.K. Sakumoto and H.S. Budd, "Run II PEM Tower Transverse Response Maps", CDF Internal Note 6181, (2002).

[54] J. Goldstein, C. Issever, T. Nelson, F. Snider and D. Stuart, "Silicon Tracking for Plug Electrons", CDF Internal Note 5970, (2002).

[55] C. Hill, J. Incandela and C. Mills, "Electron Identification in Offline Release 5.3", CDF Internal Note 7309, (2004).

[56] W.K. Sakumoto, "Event $\left|Z_{v t x}\right|<60$ Cut Acceptance for Run II", CDF Internal Note 7324, (2004). 
[57] J. Goldstein, S. Harper, P. Renton and G. Veramendi, "Very High-Pt Electron Identification", CDF Internal Note 7527, (2005).

[58] Ray Culbertson, Fermilab, private conversation.

[59] The CDF Statistics Committee, http://www-cdf.fnal.gov/physics/statistics/statistics_faq.html.

[60] T. Nelson, D. Stuart, K. Ikado, K. Maeshima, M. Karagoz Unel, "Search for New Physics in Forward High-mass Dielectrons", CDF Internal Note 6791, (2003).

[61] D. Acosta et al. (The CDF Collaboration), "Search for New Physics Using High Mass Tau Pairs from 1.96-TeV p anti-p Collisions", Phys. Rev. Lett. 95, 131801 (2005).

[62] F. Abe et al. (The CDF Collaboration), "Search for New Particles Decaying to Dijets at CDF", Phys. Rev. D, Rapid Comm., 55, R5263, (1997).

[63] V. M. Abazov et al. (The DØ Collaboration), "Search for heavy particles decaying into electron positron pairs in p-barp collisions", Phys. Rev. Lett. 87, 061802 (2001).

[64] V. M. Abazov et al. (The DØ Collaboration), "Search for New Particles in the Two-jet Decay Channel with the DØ Detector", Phys. Rev. D, Rapid Comm., 69, 111101, (2004).

[65] J. Abdallah et al. (The DELPHI Collaboration), "Search for Technicolor with DELPHI", Eur. Phys. J C 22, 17, (2001).

[66] V. M. Abazov et al. (The DØ Collaboration), "Search for Randall-Sundrum Gravitons in Dilepton and Diphoton Final States", Phys. Rev. Lett. 95, 091801 (2005).

[67] T. Binoth, J.P. Guillet, E. Pilon and M. Werlen, "A Full next-to-leading order study of direct photon pair production in hadronic collisions", Eur. Phys. J C 16, $311,(2000)$.

[68] The ATLAS Collaboration, ATLAS TDR 14, CERN/LHCC 99-14, (1999). 